\title{
DEPOSITION, CHARACTERIZATION, AND FABRICATION OF A ZINC OXIDE PIEZOELECTRIC THIN FILM MICROSPEAKER USING DC REACTIVE SPUTTERING
}

\author{
A Thesis \\ presented to \\ the Faculty of California Polytechnic State University, \\ San Luis Obispo
}

\begin{abstract}
In Partial Fulfillment
of the Requirements for the Degree

Master of Science in Engineering,

with Specialization in Materials Engineering
\end{abstract}

by

Adam Olzick

June 2012 
(C) 2012

Adam Olzick

ALL RIGHTS RESERVED 
COMMITTEE MEMBERSHIP

TITLE: DEPOSITION, CHARACTERIZATION, AND FABRICATION OF A ZINC OXIDE PIEZOELECTRIC THIN FILM MICROSPEAKER USING DC REACTIVE SPUTTERING

AUTHOR: $\quad$ Adam Olzick

DATE SUBMITTED: June 2012

COMMITTEE CHAIR: Dr. Richard Savage

Professor of Materials Engineering

California Polytechnic State University,

San Luis Obispo

COMMITTEE MEMBER: Dr. Linda Vanasupa

Professor of Materials Engineering

California Polytechnic State University,

San Luis Obispo

COMMITTEE MEMBER: Dr. Daniel Walsh

Professor of Materials Engineering

California Polytechnic State University,

San Luis Obispo 


\section{ABSTRACT \\ DEPOSITION, CHARACTERIZATION, AND FABRICATION OF A ZINC OXIDE PIEZOELECTRIC THIN FILM MICROSPEAKER USING DC REACTIVE SPUTTERING}

\section{Adam Olzick}

A piezoelectric microspeaker device that could be used in a variety of acoustic applications was designed and fabricated using a thin film $\mathrm{ZnO}$ layer that was reactively DC sputtered onto a single crystalline n-type silicon substrate. When tested the microspeaker did not produce sound due to complications in the etching process, the thickness of the diaphragms, and clamping effects. Instead, a characterization approach was taken and the structural, optical, electrical, and piezoelectric properties of the $\mathrm{ZnO}$ were investigated. Scanning electron microscopy, x-ray diffraction, and atomic force microscopy were utilized to discover the ZnO's structural properties. Using the XRD and SEM, the assputtered $\mathrm{ZnO}$ films were found to have highly c-axis oriented columnar crystals. Optical properties were determined from the reflectance spectrums obtained from a Filmetrics F20 reflectometer and were used to determine the film thickness, the optical constants, and the optical band gap of the $\mathrm{ZnO}$ thin films. Using a fourpoint probe, the as-sputtered $\mathrm{ZnO}$ films were found to be highly resistive and insulative, mainly due to voided growth boundaries between the crystals. To improve electrical conductivity and piezoelectric response, $\mathrm{ZnO}$ samples were annealed at varying temperatures in a nitrogen environment. The annealing process successfully increased the electrical conductivity and piezoelectric properties of the films. The local piezoelectric properties of the $\mathrm{ZnO}$ were 
discovered with an Asylum MFP-3D and a piezoresponse force microscopy (PFM) technique called DART-PFM. The $\mathrm{ZnO}$ films that were sputtered with 70 watts and an argon to oxygen gas ratio of 2:1 were found to have the highest $d_{33}$ piezoelectric coefficients. The $\mathrm{ZnO}$ sample that was annealed at $600^{\circ} \mathrm{C}$ for 30 minutes had the highest overall $d_{33}$ value of $4.0 \mathrm{pm} / \mathrm{V}$, which means that the $600^{\circ} \mathrm{C}$ annealed $\mathrm{ZnO}$ films would have the best chance of making a functional microspeaker. 


\section{ACKNOWLEDGEMENTS}

I'd like to thank Dr. Savage for his help and guidance throughout my project. l'd also like to thank the Materials Engineering department and all of the faculty, as well as the Dean of Research and Graduate Programs, Susan Opava, for funding my research through the purchase of conductive AFM cantilevers. My project wouldn't have been possible without her. Also, thank you to all of people that helped me along the way who are mostly gone by now: Patrick Hyland, Brian Alvarez, Josh Angell, Tim McCrone, Mike Marrujo, and Ross Gregoriev to name a few. Thanks guys, you all helped keep me motivated and I really appreciate it! l'd also like to thank my parents for supporting me through my entire school career. It was a long journey, but it was worth it. 


\section{TABLE OF CONTENTS}

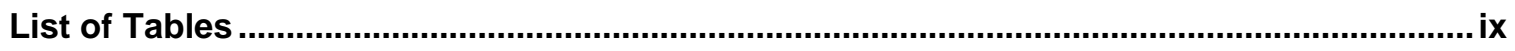

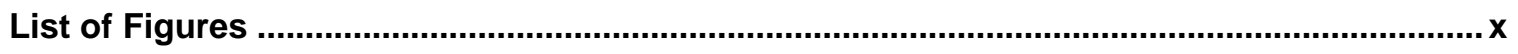

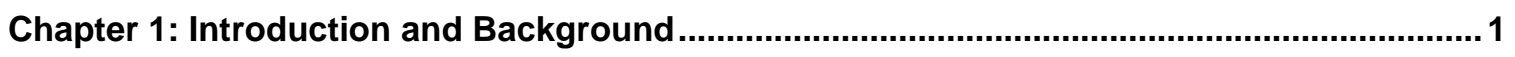

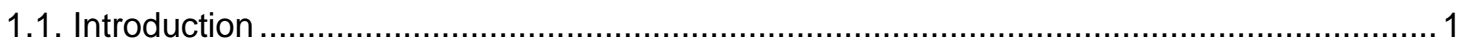

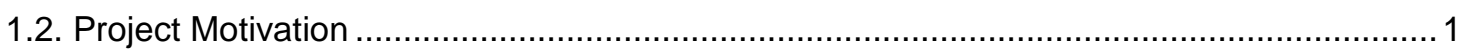

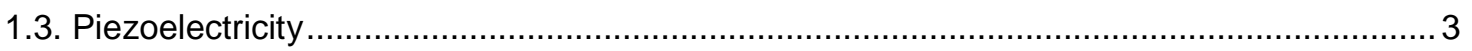

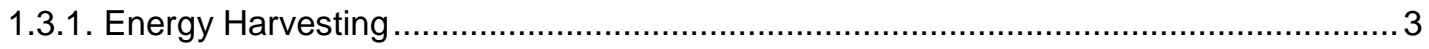

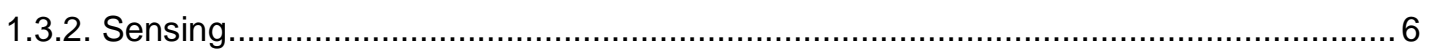

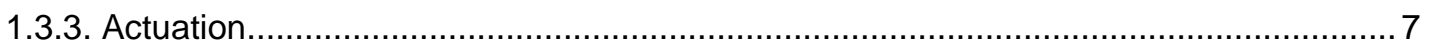

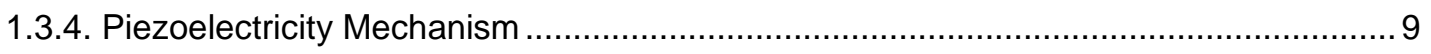

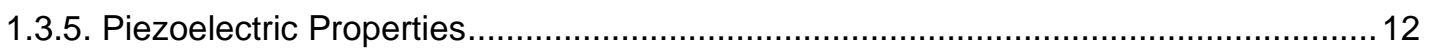

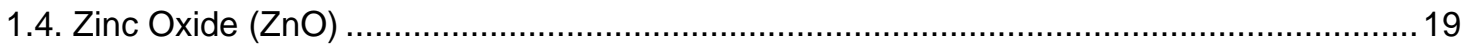

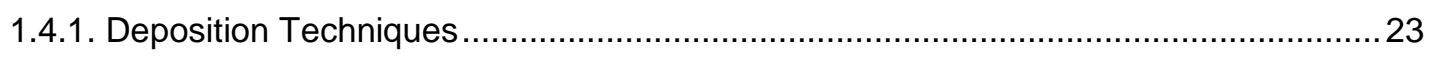

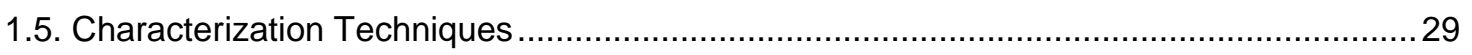

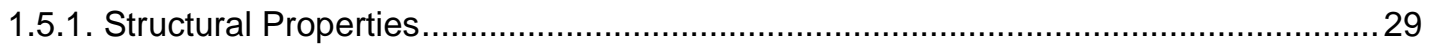

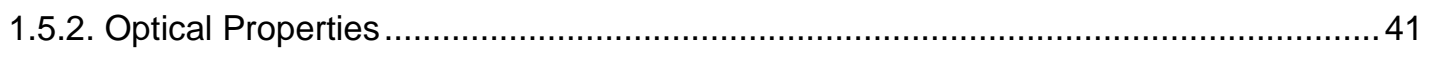

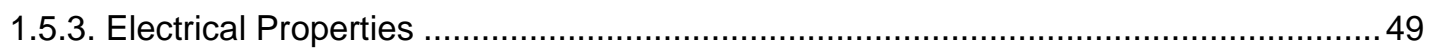

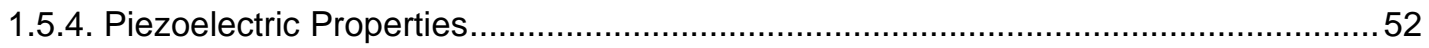

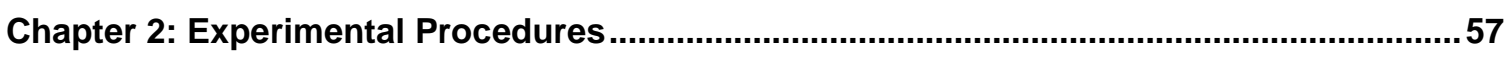

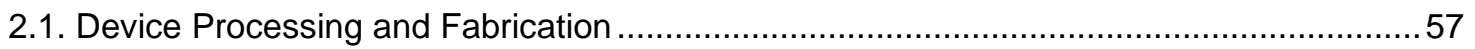

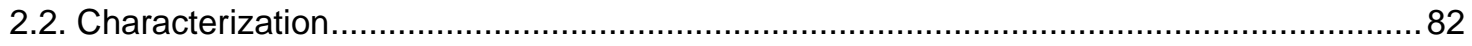

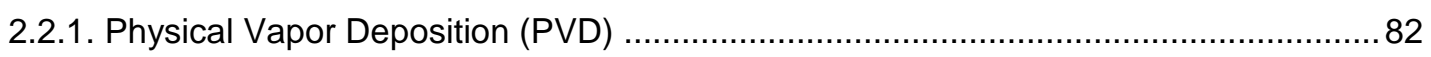

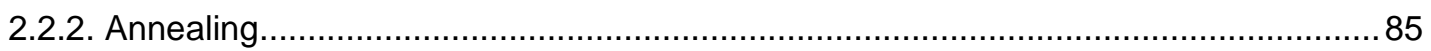

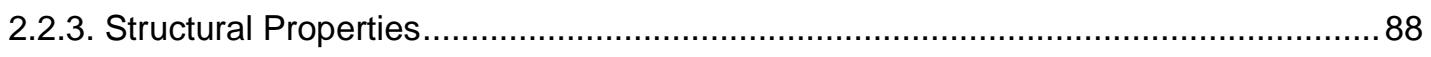

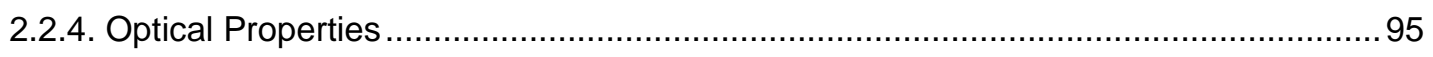

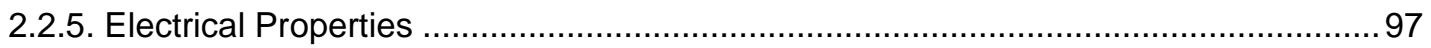

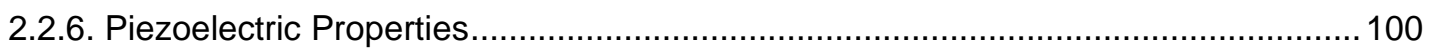

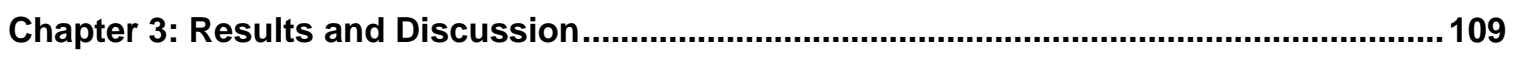

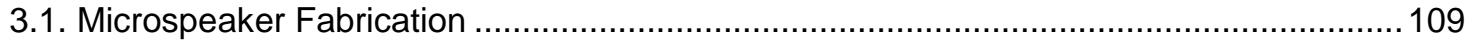

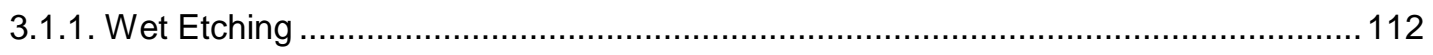

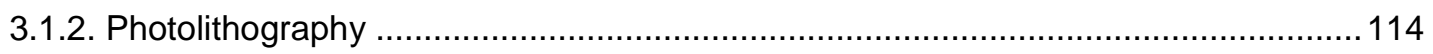

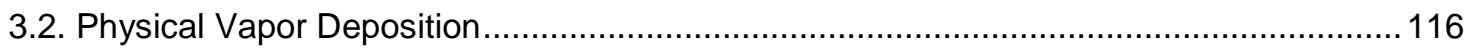

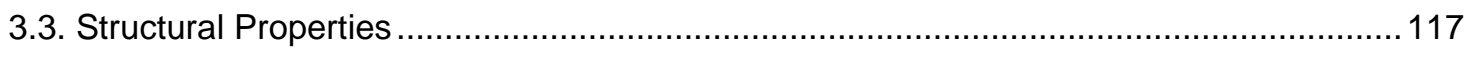

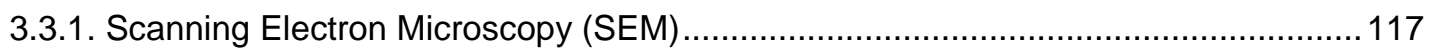

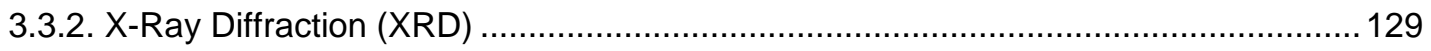

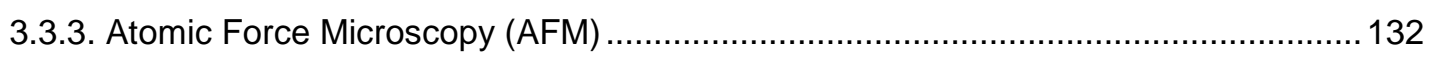

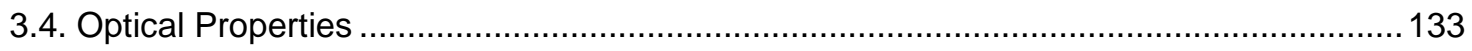




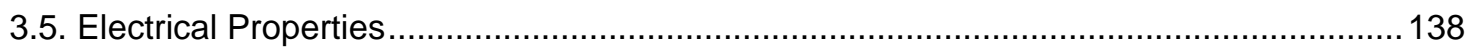

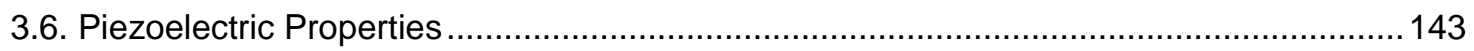

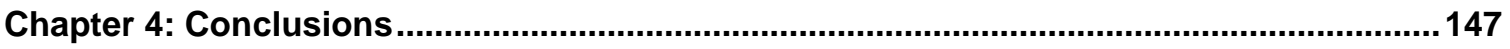

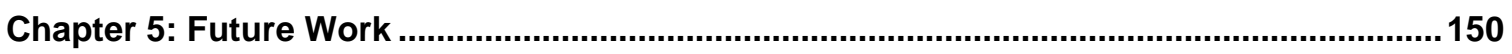

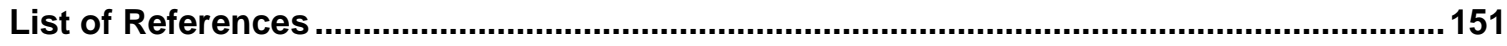

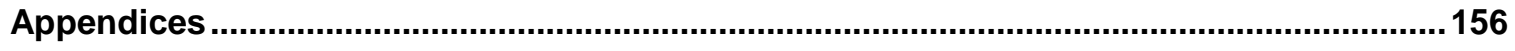




\section{LIST OF TABLES}

Table I - Common Piezoelectric, Electrical, and Physical Properties ....................................... 18

Table II - Material Properties of ZnO

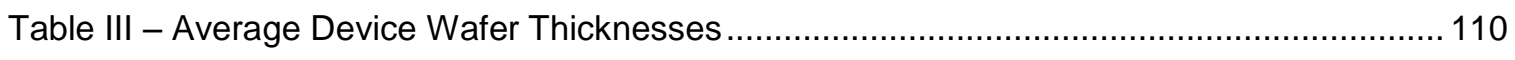

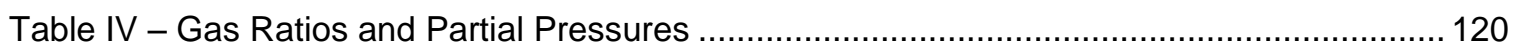




\section{LIST OF FIGURES}

Figure 1 - Piezoelectrics are unique materials that can convert electrical energy into mechanical energy or convert mechanical energy into electrical energy.

Figure 2 - The force generated by the weight of a person walking on the piezoelectric tiles creates electricity that can then be stored and used to power other devices.

Figure 3 - The material experiences the direct piezoelectric effect when a pressure is applied, resulting in an electrical potential that can be detected.

Figure 4 - The reverse piezoelectric effect results in a displacement when an electrical potential is applied to the piezoelectric material.

Figure 5 - Piezoelectrics are a dielectric material that can change its dipole moment and thus its polarization, depending on how much stress or strain is being applied to the crystal.

Figure 6 - An unstressed, neutrally charged crystal where all of the negatively charged atoms and positively charged atoms balance each other out at what is called the center of charge. When a piezoelectric crystal is put under stress, the center of charge becomes imbalanced and the positively charged atoms no longer balance with the negatively charged atoms. This creates a dipole within the crystal, which creates surface charges that can be measured.

Figure 7 - When a piezoelectric thin film is stressed, a dipole is created in all of the crystals and they collectively generate surface charges which result in an electrical potential. Even when some crystals have a different polarization direction, the cumulative voltage will simply be smaller.

Figure 8 - The coordinate system for piezoelectric materials, where the 1, 2, and 3 axes are equivalent to the $x, y$, and $z$ axis, and the 4,5 , and 6 axes are shear forces.

Figure 9 - The $d_{33}$ piezoelectric charge coefficient is used when the displacement of interest and the direction of the electric field are in the 3 direction.

Figure 10 - In the transverse mode we use the $d_{31}$ charge coefficient because the electric field is applied through the 3 direction and the displacement of interest is in the 1 direction........ 15 
Figure 11 - When a piezoelectric is in the shear mode, the $d_{15}$ piezoelectric charge coefficient is used. This kind of device would be called a thickness-shear mode actuator.

Figure 12 - When thinking of the transverse mode in piezoelectrics, think of when kneading bread dough. The dough will get smaller in the thickness direction, but will simultaneously get longer in the other direction.

Figure 13 - Zinc oxide has a hexagonal wurtzite crystal structure that is non-

centrosymmetric, which gives $\mathrm{ZnO}$ its unique piezoelectric properties.

Figure 14 - The $\mathrm{ZnO}$ thin film is deposited using physical vapor deposition (PVD), or sputtering. Sputtering occurs when ionized argon and oxygen gas collide with the surface or the zinc target, ejecting zinc atoms that react with the oxygen gas in the chamber and deposit onto the surface of the substrate.

Figure 15 - During sputtering, incident ions collide with the surface of the target material, which causes several cascading events to occur, including ion implantation, ejected target atoms, and the release of secondary electrons.

Figure 16 - Magnetron sputtering uses magnetic fields to confine the plasma close to the cathode in what is called the racetrack. This increases the sputter rate and allows for sputtering at lower pressures.

Figure 17 - A typical RF sputtering system that can sputter both non-conductive and conductive materials because of its high frequency alternating signal provided by an oscillator.

Figure 18 -When the electron beam hits the specimen's surface, secondary electrons, backscattered electrons, and characteristic $x$-rays are emitted from the specimen.

Figure 19 - A schematic of an SEM. The essential piece of an SEM is the electron beam that is thermionically emitted from a tungsten filament and is then condensed and focused onto the specimen using electromagnetic lenses. 
Figure 20 - SEM images of an opal structure with astigmatism in the a) in-focus condition, b) underfocused condition, c) over-focused condition, and d) in-focus condition without astigmatism.

Figure 21 - When incident $x$-rays penetrate the surface of a sample the electrons in the atoms scatter the $\mathrm{x}$-ray waves and send out new waves that can constructively interfere with one another in certain directions according to Bragg's law.

Figure 22 - The diffraction pattern for an annealed ZnO sample deposited on silicon. The distinctive Bragg's peak at $34.685^{\circ}$ indicates the (002) crystal plane, which also indicates that the $\mathrm{ZnO}$ crystals are c-axis oriented.

Figure 23 - One type of stylus profilometer configuration uses a pick-up to drag the stylus across the surface, and then height changes are measured using a transducer that can convert the analog signals into electrical signals

Figure 24 - In the microscopic scale, a typical surface contains macrodeviations, waviness, roughness, and finally microroughness.

Figure 25 - To measure the $\mathrm{ZnO}$ film's reflectance, an incident light is direct to the surface of the film, where it transmits into the film and reflects at both the upper and lower boundaries. The resulting reflected waves cause either destructive or constructive interference and are sent back to the spectrometer for analysis.

Figure 26 - The amplitude and period of the measured reflectance curve are dependent on the film's thickness, optical constants, and roughness.

Figure 27 - As the thickness of the film increases, so do the number of oscillations in the reflectance spectrum because of the wavelike nature of the light and the increased path length that the reflected light must take.

Figure 28 - A typical Tauc plot of ZnS, showing how the optical band gap of a material can be determined by extrapolating the linear portion of the curve to the $x$-axis. 
Figure 29 - Four-point probes are generally laid out in a linear fashion with the two outer electrodes carrying the current from the power supply and the inner electrodes sensing the changes in voltage.

Figure 30 - In a two-wire method an ohmmeter would be used to directly measure the resistance of the component, $R_{\text {subject }}$, but would include the resistance of all of the components in the circuit.

Figure 31 - The four-wire measurement method uses both an ammeter and a voltmeter to measure the resistance of a subject, thereby avoiding measurement of the other loads in the circuit.

Figure 32 - In a typical AFM, a silicon cantilever with a sharp tip is raster-scanned across the surface of a sample while the deflection and oscillation amplitude of the cantilever is measured with an optical tracking system.

Figure 33 - DART-PFM on the MFP-3D atomic force microscope involves applying two AC voltages to the tip to sample contact, which causes the surface of the piezoelectric to change shape. The cantilever's deflection and contact resonance frequency is tracked and measured using two lock-in amplifiers.

Figure 34 - The tip-to-surface contact between the piezoelectric thin film and the conductive cantilever is modeled as a damped harmonic oscillator. (a) Shows the tip-to-contact system, involving the piezoelectric film and the cantilever and the way that it oscillates when an AC voltage is applied. (b) Shows how the system is modeled as a DHO.

Figure 35 - The single crystal silicon test wafers that were used to fabricate the microspeaker structures were 4 inch wafers with $\{100\}$ orientation and n-type doping.

Figure 36 - To fabricate the microspeaker structure, the following processing steps had to be performed. 
Figure 37 - Before growing a thermal oxide on the surface of the silicon wafers, the wafers were cleaned of organic contamination by dipping them in a piranha solution at $70^{\circ} \mathrm{C}$ for 10 minutes.

Figure 38 - After the wafers were cleaning in piranha, the native oxides on the surface of the silicon wafers were etched away by dipping them in BOE for 10-20 seconds.

Figure $39-\mathrm{A} \mathrm{SiO}_{2}$ layer was grown on the silicon wafers using a thermal oxidation furnace that was injected with water vapor, allowing the oxidation of the silicon's surface.

Figure 40 - A Filmetrics F20 Reflectometer, which uses spectral reflectance, was used to measure the thickness of the silicon dioxide film on the wafers.

Figure 41 - The thermally grown silicon dioxide film on the silicon wafers acts as protection for the silicon during the etching process and allows the patterning of the diaphragms.

Figure 42 - Positive photoresist was spun onto the silicon wafers using a Laurell

Technologies WS-400B-6NPP spin coating system.

Figure 43 - Positive photoresist was spun onto the surface of the silicon device wafers in order to protect the surface of the oxide from the BOE.

Figure 44 - The positive photoresist was masked and exposed to UV light using a Canon PLA-501 FA aligner with a light integral of 5.5.

Figure 45 - To etch the microspeaker diaphragms in the silicon, the follow mask was used to pattern the surface. The mask features $4 \mathrm{~mm}, 5 \mathrm{~mm}$, and $7 \mathrm{~mm}$ sized squares surrounded by scribing channels.

Figure 46 - After being exposed to UV light from the aligner, the photoresist (PR) was developed in a Microposit CD-26 solution for 10 minutes.

Figure 47 - Overexposing and underexposing the photoresist can cause undesirable features to appear in the resulting pattern. 
Figure 48 - After the photoresist was developed and hard baked, the silicon dioxide was etched away by BOE for several minutes.

Figure 49 - Before etching the exposed silicon, the photoresist was stripped away using the Microposit Remover 1165 resist stripping solution.

Figure 50 - The $25 \%$ TMAH silicon wet etching solution was heated to $80^{\circ} \mathrm{C}$ using a resistive heating element and a magnetic stirring hotplate.

Figure 51 - After deep wet etching the silicon, a thin silicon layer was all that was left. The $\mathrm{ZnO}$ structure was deposited on the other side of the wafer to cause oscillations of the silicon layer.

Figure 52 - The silicon device wafers were delicate after deep etching and had to be handled with care. In certain locations around the wafer some of the diaphragms were etched all of the way through

Figure 53 - A thin film of aluminum was deposited onto the silicon wafer to act as the bottom electrode of the $\mathrm{ZnO}$ actuator structure.

Figure 54 - To spin coat more photoresist onto the wafer for lithography of the deposited aluminum electrodes, aluminum foil had to be taped to the backside of the wafer.

Figure 55 - The first layer of the $\mathrm{ZnO}$ actuator structure consisted of patterned aluminum electrodes that would provide electrical access to the bottom of the $\mathrm{ZnO}$ film.

Figure 56 - After sputtering aluminum on the silicon wafers, photoresist was patterned on top of the aluminum and then the aluminum was etched away with an aluminum etchant.

Figure 57 - The $\mathrm{ZnO}$ was patterned with photoresist into $4 \mathrm{~mm}, 5 \mathrm{~mm}$, and $7 \mathrm{~mm}$ squares

directly over the diaphragm structure.

Figure 58 - After depositing $\mathrm{ZnO}$, spin coating on photoresist, and patterning the photoresist, the $\mathrm{ZnO}$ was etched away with a weak acetic acid aqueous solution. 
Figure 59 - The last layer of the structure was an aluminum layer that acted as the top electrode of the microspeaker. This layer is similar to the first aluminum electrode layer, except oriented in another direction to avoid shorting with the first aluminum layer.

Figure 60 - The complete microspeaker structure was obtained after sputtering and etching the last layer of aluminum.

Figure 61 - In order to create a microspeaker, the $\mathrm{ZnO}$ thin film must be able to oscillate a thin silicon diaphragm that was etched into a $100 \mathrm{~mm}$-type single crystalline silicon wafer. 81 Figure 62 - Physical vapor deposition of aluminum and zinc oxide thin films is performed using the Torr CRC-150. Zinc oxide is deposited by flowing high purity oxygen gas into the chamber using the gas flow controller on the right.

Figure 63 - The diced pieces of n-type silicon were ultrasonically cleaned for 15 minutes in an acetone bath and then rinsed with isopropyl alcohol, DI water, and then dried with nitrogen.

Figure 64 - The JOEL JSM-6390 scanning electron microscope was used to take topographical and cross-section images of the $\mathrm{ZnO}$ samples.

Figure 65 - To obtain cross-sectional and topographical images, $\mathrm{ZnO}$ samples were diced and placed into slotted and flat sample mounts and then tightened into place into a multisample holder with set screws.

Figure 66 - In order to determine the quality of the crystals and their orientation, X-ray diffraction (XRD) was performed on the $\mathrm{ZnO}$ thin film samples.

Figure 67 - The $\mathrm{ZnO}$ samples were placed onto clay and mounted onto a three-pronged sample mount which was leveled with a thick glass plate.

Figure 68 - An Ambios XP-1 stylus or surface profilometer was used to calculate the thicknesses of the $\mathrm{ZnO}$ thin films. 93 
Figure 69 - As the stylus approaches the surface (on the right), a shadow of the stylus will start to appear on the left hand side of the screen. As the stylus gets closer to the sample's surface, the two will come closer together.

Figure 70 - To get a better representation of the change in height, the analysis cursors were resized along the surface of the $\mathrm{ZnO}$ and the silicon. The spike in the step height seen in the image above is a result of the removal of the Kapton tape creating an uneven edge on the $\mathrm{ZnO}$.

Figure 71 - Reflectance spectrums of the $\mathrm{ZnO}$ thin films were calculated and measured with

a Filmetrics F20 thin-film analyzer.

Figure 72 - The electrical resistivity of the $\mathrm{ZnO}$ films was attempted using a Signatone SP4 four-point probe head.

Figure 73 - The MFP-3D AFM system is enclosed in an acoustic and vibration damping housing that enables the measurement of incredible small features without the effects of noise. 101

Figure 74 - The conductive cantilevers that were used in the PFM measurements were Olympus AC240TM ElectriLevers purchased from Asylum Research.

Figure 75 - The deposited conductive layer on the tip can easily be worn during imaging. 103

Figure 76 - The DART Panel is a unique panel for DART-PFM that allows contact-resonance tuning to be performed and the drive voltage and scanning parameters changed.

Figure 77 - The contact resonance is tuned by increasing the voltage on the tip until the received signal is over $5 \mathrm{mV}$. 106

Figure 78 - The AmDR image that is generated after pressing the Calc SHO Parms button represents the local piezoelectric response of the crystals.

Figure 79 - The raw data is inputted into histogram form, where the mean displacement value can be determined by using a fitting the data with a Lorentzian distribution. 108 
Figure 80 - Instead of a complicated photolithography process with spin coating and photoresist, a working device could be made with just Kapton tape acting as the mask.

Figure 81 - Cleaning the cathode shield and cathode holder will help improve the quality of the deposited films and the plasma's stability. A clean sputtering machine is a happy sputtering machine.

Figure $82-\mathrm{ZnO}$ crystals that are not oriented in the c-axis direction will still be piezoelectric in nature, but will be oriented in a direction that will result in smaller piezoelectric coefficients.. 117 Figure 83 - The columnar structure of the $\mathrm{ZnO}$ crystals can be seen in this micrograph of a sample that was sputtered for 60 minutes at $100 \mathrm{~W}$ with a $1: 1 \mathrm{Ar}: \mathrm{O}_{2}$ gas ratio.

Figure 84 - This incredibly thin $\mathrm{ZnO}$ film that was sputtered for 30 minutes at $70 \mathrm{~W}$ with a 1:2 gas ratio was difficult to image because of how thin it was. However, a columnar structure can still be seen in the crystals.

Figure 85 - The effect of gas ratios on $\mathrm{ZnO}$ that has been sputtered for 60 minutes at $100 \mathrm{~W}$ at (a) 2:1 gas ratio, (b) 1:1 gas ratio, (c) 1:2 gas ratio, and (d) 1:3 gas ratio.

Figure 86 - Effect of gas ratios on $\mathrm{ZnO}$ samples that have been sputtered for 45 minutes at $100 \mathrm{~W}$ at a, (a) 2:1 gas ratio, (b) 1:1 gas ratio, (c) 1:2 gas ratio, and (d) 1:3 gas ratio.

Figure 87 - The effect that gas ratios had on the structural properties of $\mathrm{ZnO}$ sputtered for 30 minutes at $100 \mathrm{~W}$ at a (a) 2:1 gas ratio, (b) 1:1 gas ratio, (c) 1:2 gas ratio, and (d) 1:3 gas ratio.

Figure 88 - This typical hysteresis curve for sputtering with a metal target in a reactive gas shows how an insulating compound can form on the surface of the target and causing "poisoning" by decreasing sputter rates.

Figure 89 - Topographical image of $\mathrm{ZnO}$ sputtered for 60 minutes at $100 \mathrm{~W}$ with a gas ratio of (a) 2:1, (b) 1:1, (c) 1:2, and (d) 1:3. 125

Figure 90 - SEM micrographs of 1:1 $\mathrm{Ar}: \mathrm{O}_{2}$ deposited $\mathrm{ZnO}$ annealed for 30 minutes at varying temperatures. (a) As sputtered $\mathrm{ZnO}$ control sample with no annealing, (b) $\mathrm{ZnO}$ annealed at 
$600^{\circ} \mathrm{C}$, (c) $\mathrm{ZnO}$ annealed at $800^{\circ} \mathrm{C}$ for 30 minutes, (d) $\mathrm{ZnO}$ annealed at $800^{\circ} \mathrm{C}$ for 1 hour, (e) $\mathrm{ZnO}$ annealed at $1000^{\circ} \mathrm{C}$

Figure 91 - ZnO thin films deposited with a 1:1 gas ratio were annealed in a high-purity nitrogen environment at various temperatures and times: (a) as-sputtered, (b) $600^{\circ} \mathrm{C}$ anneal for 30 minutes , (c) $800^{\circ} \mathrm{C}$ anneal for 30 minutes, (d) $800^{\circ} \mathrm{C}$ anneal for 1 hour, (e) $1000^{\circ} \mathrm{C}$ anneal for 30 minutes.

Figure 92 - All 24 as-sputtered samples and the four additional annealed samples are shown in this XRD graph. The four annealed samples had much higher intensities and also showed a shift in the (002) peak angle, indicating relaxation of the crystals.

Figure 93 - The 2:1 gas ratio $\mathrm{ZnO}$ sample had the highest intensity (002) peak at $34.302^{\circ}$, indicating that the crystal is strongly c-axis oriented.

Figure 94 - A graph relating the gas ratios to the FWHM found through XRD measurements shows that as the oxygen partial pressure increases, the FWHM tends to increase as well. The 90 minute sputter represents the four 1:1 ratio, $100 \mathrm{~W}$ annealed $\mathrm{ZnO}$ samples

Figure 95 - The surface topography of a $\mathrm{ZnO}$ sample that was sputtered for 60 minutes at 100 W with a 2:1 gas ratio was obtained using the AC mode on an MFP-3D AFM.

Figure 96 - The reflectance spectrum for a ZnO sample obtained from a Filmetrics F20 thinfilm analyzer.

Figure 97 - The index of refraction was determined from reflectance data and the values for all of the $100 \mathrm{~W}$ samples are shown in this graph. The index of refraction tends to decrease exponentially as the wavelength of the light increases.

Figure 98 - The extinction coefficient of the as-sputtered $\mathrm{ZnO}$ samples was obtained from reflectance data.

Figure 99 - The Tauc plot is used to calculate the optical band gap of a material by extending the linear portion of the exponential graph to the $\mathrm{x}$-axis. Where the extension hits the $\mathrm{x}$-axis is the band gap of the material. 
Figure 100 - The band gap for the $\mathrm{ZnO}$ sample annealed at $800^{\circ} \mathrm{C}$ for 1 hour was the only sample that had a band gap in the typical range for $\mathrm{ZnO}$.

Figure 101 - Unlike the highly resistive as-sputtered $\mathrm{ZnO}$, the resistivity of the annealed $\mathrm{ZnO}$ samples was able to be determined using the 4-point probe method....

Figure 102 - The $d_{33}$ values were calculated for all of the as-sputtered and the four annealed samples. The sample annealed at $600^{\circ} \mathrm{C}$ for 30 minutes resulted in the highest piezoelectric response.

Figure 103 - A main effects plot was generated from Minitab and the data from the 24 assputtered $\mathrm{ZnO}$ samples and the four annealed $\mathrm{ZnO}$ samples. High oxygen partial pressures tended decrease the piezoelectric response of the $\mathrm{ZnO}$. A 90 minute sputter was performed only on the four annealed samples.

Figure 104 - The conductive tips experienced severe degradation after only a few scans. The top SEM micrograph shows an image of an unused conductive tip and the bottom micrograph shows the same tip after only a few PFM scans. 


\section{Chapter 1: Introduction and Background}

\subsection{Introduction}

There are many different types of materials with unique properties that can change if exposed to certain conditions and environments, for example ferromagnetic materials, semiconducting materials, pyroelectric materials, and piezoelectric materials. Shape memory alloys can change the shape of their crystal structure with the addition of heat; ferroelectric materials possess a spontaneous polarization that can be reversed by applying an external electric field; pyroelectric materials can create electricity when they are heated or cooled; and piezoelectric materials can convert mechanical energy into electricity. Surprisingly, these materials come from nature and are altered by our hands into forms that can be useful to us. For example, we can deposit very thin films of these materials onto substrates to be used in micro-sized devices or microelectromechanical systems (MEMS). These devices can be found in many modern consumer electronics such as smartphones and tablets, as well as in all other fields of industry where small sensors or actuators are required. ${ }^{1}$

\subsection{Project Motivation}

Alternative energy and the different ways that we can utilize and harvest wasted energy are a fascinating area of technology that will benefit future generations and will help us improve our energy efficiency. When looking for potential projects, piezoelectricity caught my interest because of its ability to be used as an alternative source of energy. Piezoelectric materials are materials 
that we can use to harvest energy that would otherwise be wasted. Piezoelectrics can also be used as sensors to detect changes in acceleration or force or they can be used to actuate MEMS devices at the micron scale. Initially, the goal of the project was to fabricate a droplet generator with silicon and the piezoelectric material $\mathrm{ZnO}$. The droplet generator would eject picometer sized ink droplets from a reservoir in extremely precise and consistent amounts. Instead of typical ink in an inkjet printer, the idea was to use a liquid photovoltaic polymer that could be printed onto surfaces. For simplicity's sake, the project evolved into the fabrication of a microspeaker because the sound pressure level (SPL) of the microspeaker could be measured and used to calculate the displacement of the microspeaker's diaphragm. This relationship between SPL and displacement could be used as a way to characterize the $\mathrm{ZnO}$ and its piezoelectric response.

However, once I encountered difficulties in the device's fabrication, I discovered that without knowing more about the as-sputtered zinc oxide film and its properties, I could not fabricate a functional microspeaker. This realization caused my thesis to change from the fabrication of a device to the characterization of $\mathrm{ZnO}$. Characterization of the $\mathrm{ZnO}$ would allow me to determine which sputtering parameters would give the most desirable piezoelectric response and material properties for a microspeaker device. If I did not know more about the $\mathrm{ZnO}$ that I was depositing, I could not possibly make a working device. Without knowing how piezoelectric my films were, I could not know which parameters were ideal in the sputtering process. For example, the sputtering parameters that I used when fabricating the microspeakers could have 
had the weak piezoelectric coefficients and therefore could not cause enough displacement to actuate the silicon diaphragms. Ideally, a student in the future will be able to utilize this discovered information and will be able to fabricate a working device and expand the university's fabrication capabilities.

\subsection{Piezoelectricity}

The material that will be characterized and used to actuate a microspeaker is a piezoelectric material called zinc oxide ( $\mathrm{ZnO})$. The unique ability piezoelectric materials possess is the ability to convert mechanical energy into electrical energy, which is called the piezoelectric effect. In addition, a piezoelectric material can also convert electrical energy into mechanical energy, which is known as the reverse/converse piezoelectric effect (Figure 1). These abilities allow piezoelectric materials to be used in three different ways, (1) as an energy harvesting material, (2) as a sensing material, or (3) as an actuating material.

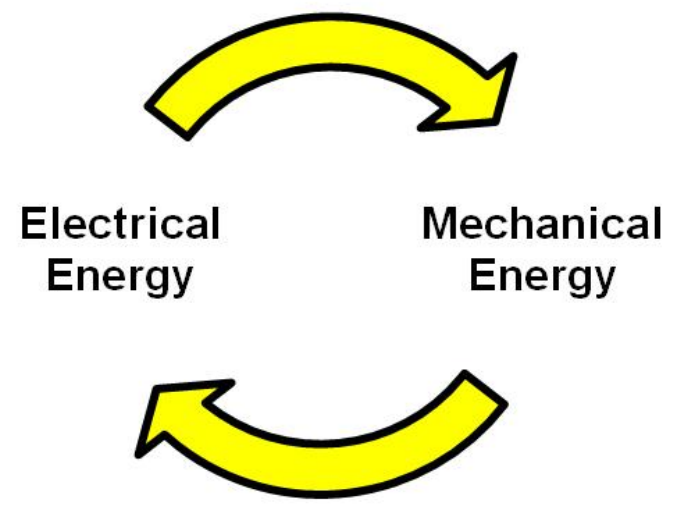

Figure 1 - Piezoelectrics are unique materials that can convert electrical energy into mechanical energy or convert mechanical energy into electrical energy.

\subsubsection{Energy Harvesting}

Energy harvesting involves the generation of electricity from external vibrations which are transferred to the piezoelectric material and can be collected 
and stored. Once a force is applied to a piezoelectric material, an electrical charge is generated that is proportional to the amount of force applied.

Therefore, a greater force applied to the piezoelectric material will result in a larger electrical charge and a smaller force will result in a smaller electrical charge. If these electrical charges are repeatedly generated and stored, such as in a battery, then the electrical energy can be accumulated and used at a later time to power a device.

This type of electrical harvesting can be used to gather energy that would be otherwise wasted. Piezoelectricity is currently being utilized by the Japanese company, East Japan Railway. The company has placed piezoelectric devices in the floors of a passageway in a Tokyo Station multiple times from 2008 to 2009 to harvest wasted energy in locations of high traffic (Figure 2) ${ }^{2}$ The force of pedestrians walking over piezoelectric devices creates electrical energy that can be stored and used to power lighting in the station.

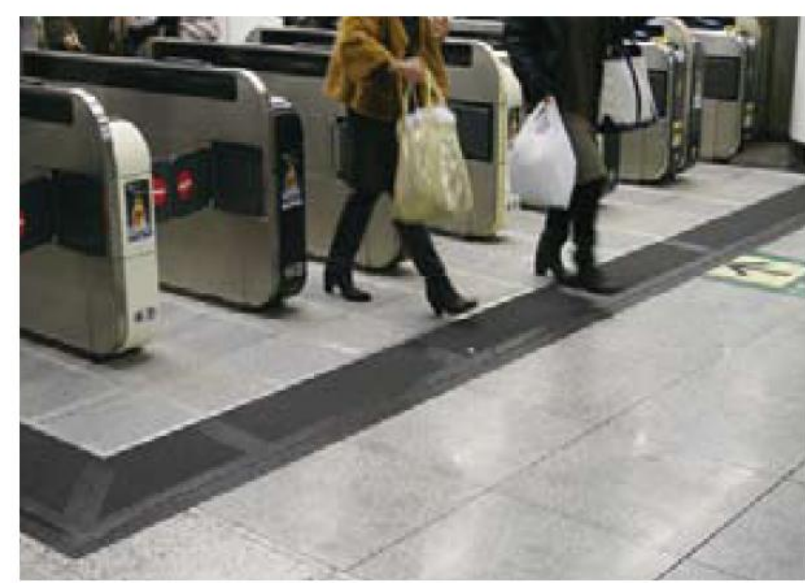

Demonstration experiment at Tokyo Station

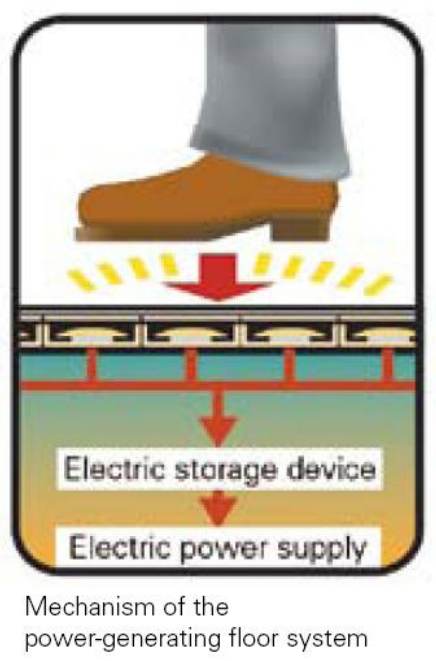

power-generating floor system

Figure 2 - The force generated by the weight of a person walking on the piezoelectric tiles creates electricity that can then be stored and used to power other devices. ${ }^{2}$ 
The efficiency of the conversion is determined by the type of piezoelectric material that is used and the quality of the piezoelectric material. Higher quality piezoelectrics will result in greater efficiency and more electricity from the same amount of force. The amount of energy that will be converted can be characterized by what are called piezoelectric coefficients of the material. There are many different types of piezoelectric coefficients because piezoelectric materials are anisotropic and do not act the same way in different structural orientations. These piezoelectric coefficients will be explained in greater detail in section 1.3.5, Piezoelectric Properties. 


\subsubsection{Sensing}

The second useful property of piezoelectric materials is their ability to be used as a sensor. This means that piezoelectrics can be used to detect extremely small changes in force, acceleration, pressure, strain, etc. These small changes in force, acceleration, pressure, or strain result in an electrical charge that can be detected, measured, and analyzed. Any type of movement that can cause the piezoelectric material to change shape can be used to make the piezoelectric a sensor (Figure 3).

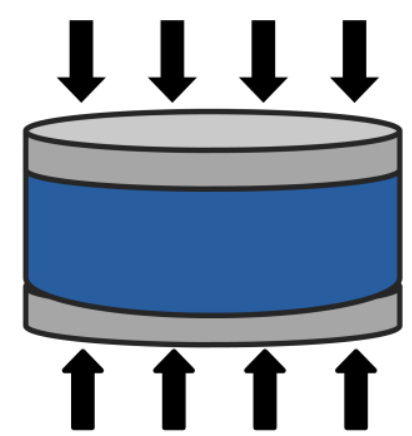

Apply Pressure

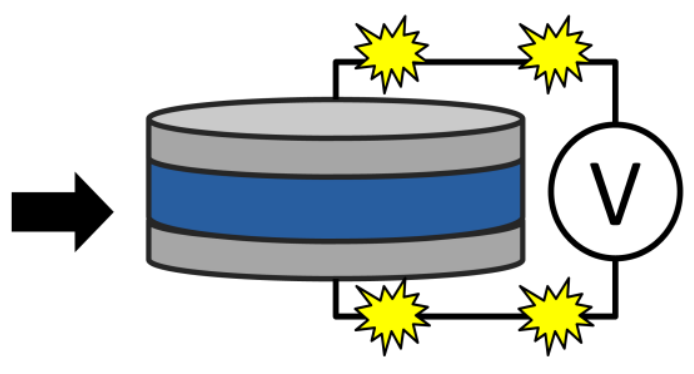

Result: Voltage

Figure 3 - The material experiences the direct piezoelectric effect when a pressure is applied, resulting in an electrical potential that can be detected.

Piezoelectric materials are unique in that the electrical charge generated can be as small as the force that is used to generate that charge, which means that if a piconewton of force is applied to the piezoelectric material an electrical charge proportional to a piconewton of force will be generated. For this reason, piezoelectric sensors are incredibly accurate and precise. The only issue is having equipment capable of detecting such small changes in electrical charge.

Piezoelectrics can be used as force sensors, accelerometers, chemical sensors, gas sensors, surface acoustic wave devices, etc. In order to be a 
sensor with extremely high sensitivities, piezoelectric thin films are often deposited onto silicon substrates that are bulk micromachined into various different structures. A well known structure is the microcantilever, which is etched out of silicon and then piezoelectric materials like PZT, $\mathrm{ZnO}$, and AIN are deposited onto the surface. Contacts and sometimes another coating that attracts certain molecules are also deposited, resulting in a small microelectromechanical system, also known as a MEMS device. These cantilevers are oscillated at their resonant frequency, which is often in the kilohertz range, and when a molecule that corresponds to the coating falls and attaches onto the cantilever, the resonant frequency of the cantilever will change slightly. This small change in resonant frequency can be detected and allows the cantilever to act as a chemical sensor. Another example of a sensor is a simple microphone. If a piezoelectric film is deposited onto a very thin diaphragm and sound pressure waves are directed toward the diaphragm, then the sound pressure will cause the diaphragm to displace, which will cause the piezoelectric material to generate electric charge, which can be detected and interpreted. The larger the displacement, the larger the electric charge generated and the greater the sound pressure that was applied.

\subsubsection{Actuation}

The third ability of piezoelectric materials is actuation, where electricity can be applied to the piezoelectric material and the piezoelectric will change its shape and induce strain in its crystals (Figure 4). This occurs because of the uneven arrangement of the atoms in its crystals. Typically, in normal non-piezoelectric 
materials, the charges in their crystals are balanced and cancel out, making the crystal neutrally charged when pressure is applied. On the other hand, when pressure is applied to a piezoelectric material, the crystal become polarized and the center of charge becomes heavily imbalanced and creates what is called a dipole in the crystal. This imbalance of charge is the essential mechanism that allows piezoelectrics to behave as they do.

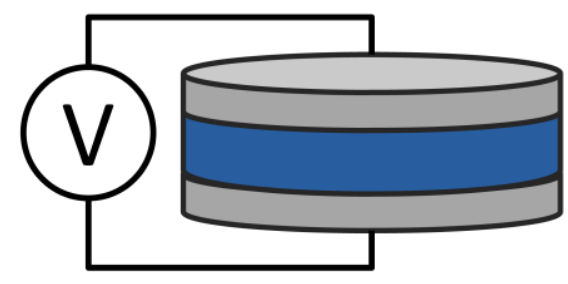

Apply Voltage

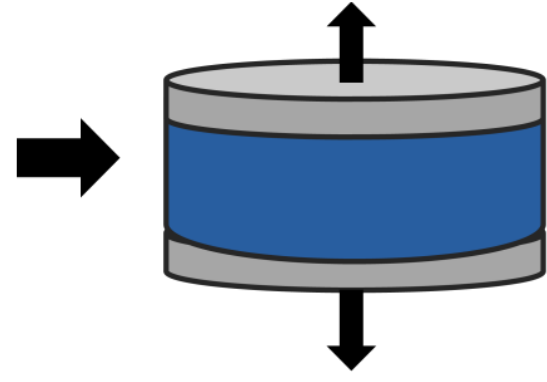

Result: Displacement

Figure 4 - The reverse piezoelectric effect results in a displacement when an electrical potential is applied to the piezoelectric material.

Similar to its sensing abilities, piezoelectric materials can generate strain or displacement in amounts proportional to the amount of electricity that is applied to the piezoelectric material. Because piezoelectrics move proportionally to how much electrical charge is applied, piezoelectrics are often used to make incredibly small and precise movements, such as in atomic force microscopy (AFM). The amount of displacement is only limited by how small of an electrical charge that can be applied to the piezoelectric. In applications where piezoelectrics are used for actuation, often multiple plates of piezoelectric ceramics are stacked on top of each other and wired in parallel into piezoelectric stacks. Doing this increases the capacitance of the device and lowers the voltage 
requirements to cause displacement. The the mechanism of piezoelectricity is explained in more detail in section 1.3.4.

Piezoelectrics can also be used as the actuator for inkjet printing, where small displacements of a piezoelectric thin film cause a small decrease in volume of a reservoir, that small decrease in volume causes the ejection of an ink droplet that can have volumes in the picometer range. Similar to how a piezoelectric can be used as a microphone, it can also be used as a speaker, but instead of waiting for a sound pressure waves to hit the diaphragm, an AC current is applied to the piezoelectric thin film, which causes the diaphragm to move and oscillate and resulting in sound waves. This speaker device and the deposition of the piezoelectric thin film that actuates it will be the main focus of this thesis. It will be used as a way to measure the performance of the deposited $\mathrm{ZnO}$ thin film.

\subsubsection{Piezoelectricity Mechanism}

The word piezoelectricity comes from Hermann Henkel and the Greek word "piezen," which means "to press." Piezoelectricity originates from the arrangement of its atoms in its crystals and requires what is called a noncentrosymmetric crystal structure. The study of these arrangements of atoms in a crystal is called crystallography. Fundamentally, a piezoelectric material fits under the broad category of dielectrics. In crystallography there are a total of 32 different crystal classes. Of those 32 crystal classes, 11 crystals are centrosymmetric and therefore cannot become piezoelectric. Of the other 21 non-centrosymmetric crystal classes, 20 of them are piezoelectric, 10 of which

are also pyroelectric (Figure 5). ${ }^{3}$ A piezoelectric material is a type of dielectric 
material, which means that it relies on the polarization of its molecules to function as a piezoelectric.

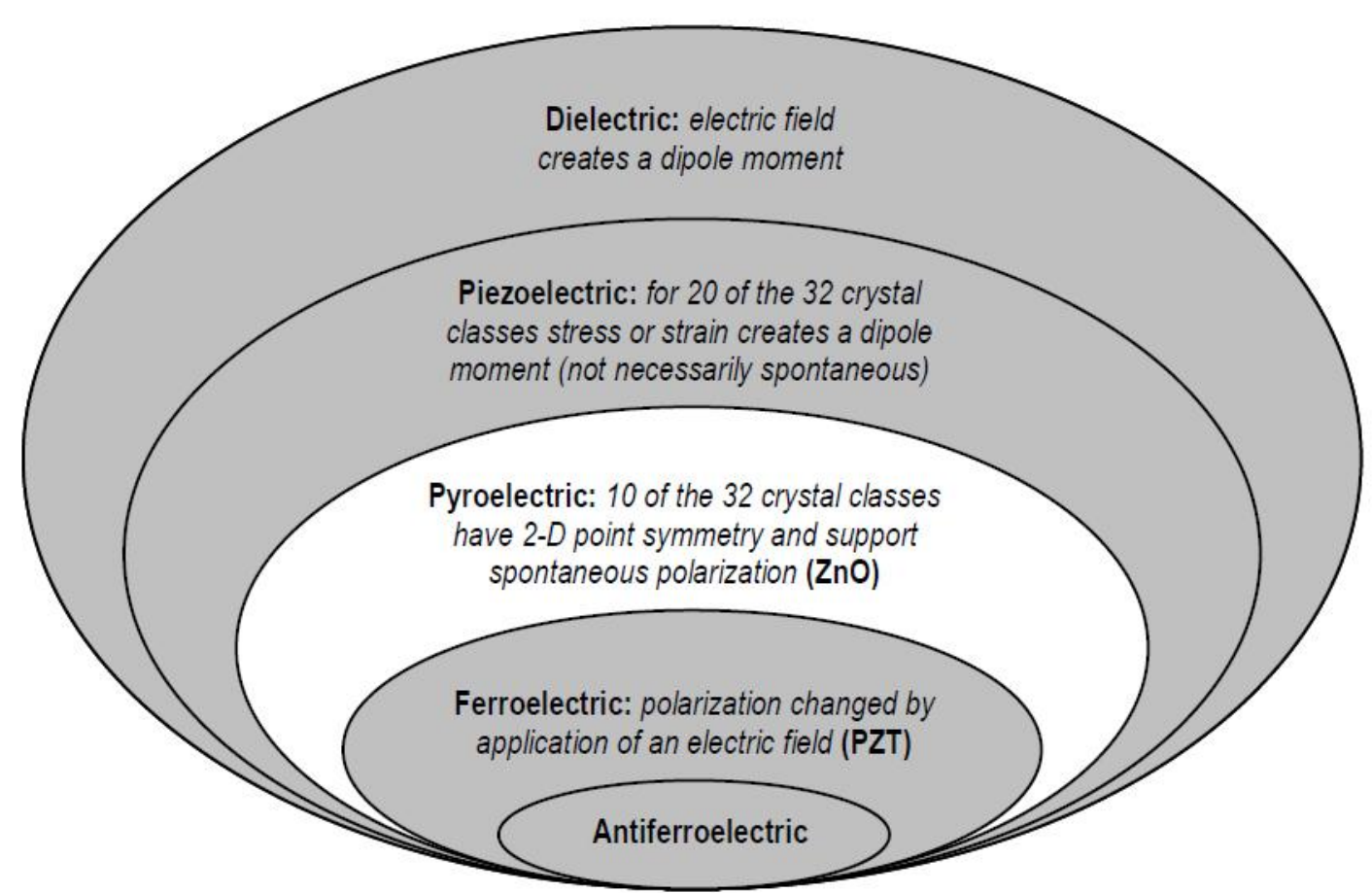

Figure 5 - Piezoelectrics are a dielectric material that can change its dipole moment and thus its polarization, depending on how much stress or strain is being applied to the crystal.

In a typical centrosymmetric crystal structure, for example a body-centered cubic crystal structure, the crystal structure is neutral and the charges are balanced, even when strained and stressed. In all crystal structures there is what is called a center of charge. Similar to how an object has a center of balance where at some location all of the weight is balanced, a crystal structure has what is called center of charge. This center of charge is where all of the positively charged atoms are in balance with all of the negatively charged atoms, making the crystal neutrally charged (Figure 6). When a pressure is applied to a piezoelectric crystal structure, it essentially puts the center of charge off balance and causes the crystal to no longer be neutrally charged. Since the center of 
charge is separated from each other, the positively charged atoms and negatively charged atoms are no longer being balanced, which causes the formation of what is called a dipole. A dipole is created when one side of the molecule is more positive or negative than the other side (Figure 6).

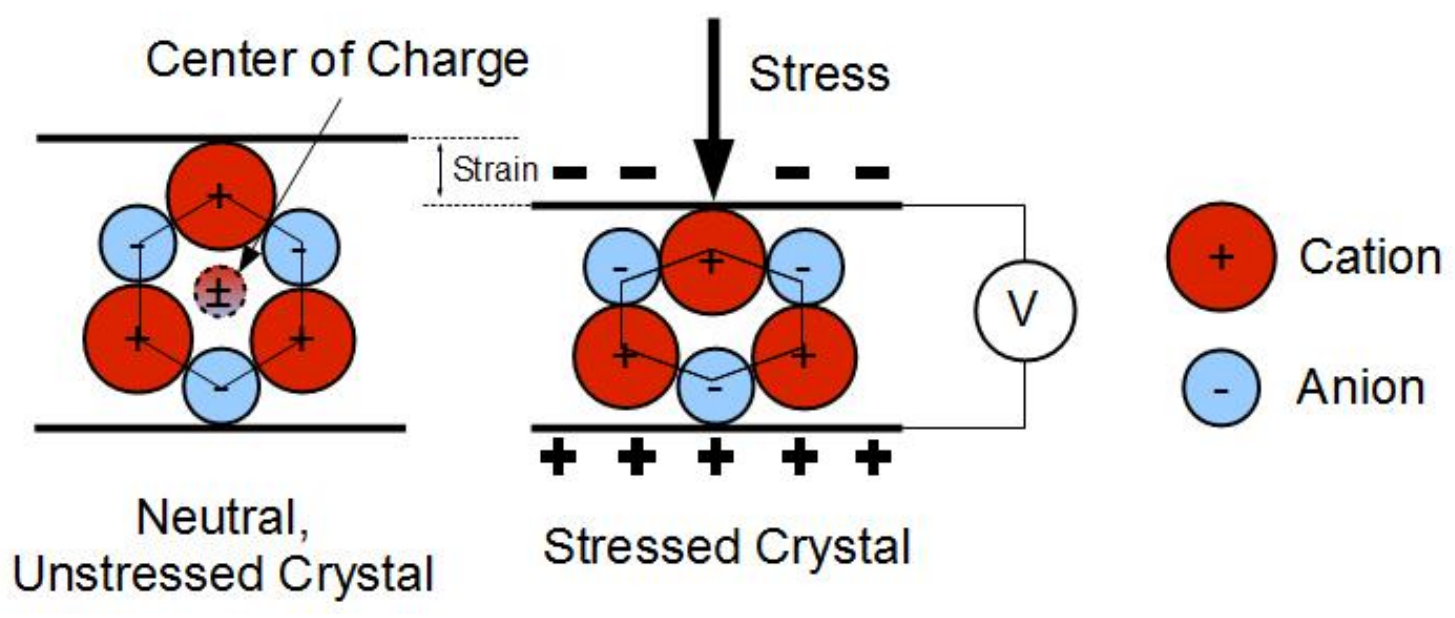

Figure 6 - An unstressed, neutrally charged crystal where all of the negatively charged atoms and positively charged atoms balance each other out at what is called the center of charge. When a piezoelectric crystal is put under stress, the center of charge becomes imbalanced and the positively charged atoms no longer balance with the negatively charged atoms. This creates a dipole within the crystal, which creates surface charges that can be measured.

When this occurs, we call this crystal structure non-centrosymmetric because when a stress is applied to the crystal, the charges do not balance and the crystal is no longer neutral. This is the same effect that occurs in a water molecule, where the oxygen end of the water molecule is partially negative and the hydrogen side is partially positive. This creates what is called a dipole moment, which points towards the more negative pole and gives water its polarity. Electrical dipoles inherently form electric fields, the potential of which are based upon the distance between the anion (-) and cation (+). Because a dipole's electric field intensity is proportional to the dipole moment, a change in the dipole moment will result in a change in the intensity of the electric field. ${ }^{3}$ 
Piezoelectricity comes from this changing electric field, which results in an electrical potential. When all of these crystals align in a certain direction, the dipole moments accumulate in the piezoelectric material and create a polarization direction. When a stress is applied it generates an electric field and as a result, a mechanical energy is converted into an electrical energy.

Piezoelectrics are typically used in dynamic situations because the charge or displacement is only generated with a changing electric field. In thin films, each crystal will generate a surface charge that will be added together cumulatively (Figure 7). If there are crystals with different orientations then the generated voltage will simply be lower than if all the crystals were oriented in the same direction.

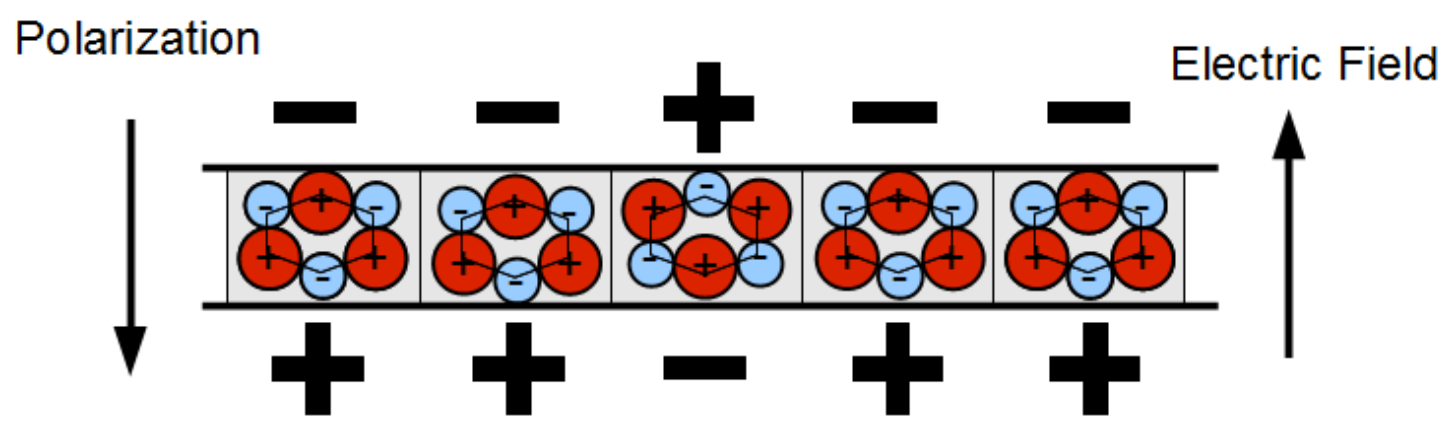

Figure 7 - When a piezoelectric thin film is stressed, a dipole is created in all of the crystals and they collectively generate surface charges which result in an electrical potential. Even when some crystals have a different polarization direction, the cumulative voltage will simply be smaller.

\subsubsection{Piezoelectric Properties}

Piezoelectric materials are anisotropic and thus behave differently in different orientations. For example, a force or voltage applied to a piezoelectric crystal oriented in the z-direction will give a different voltage than a crystal grown in another direction or orientation. The tensor notation, which is explained extensively in the 1987 IEEE Standard on Piezoelectricity, is used to ensure 
consistency in the way that piezoelectric materials and their properties are characterized and quantified. ${ }^{4}$

In the piezoelectric coordinate system, the numbers 1,2 , and 3 are used to represent the $x, y$, and $z$ axes and the numbers 4,5 , and 6 represent shear about the axes (Figure 8$).{ }^{5}$ This coordinate system is essential for piezoelectric properties and can give insight into the polarization direction and direction of the electric field.

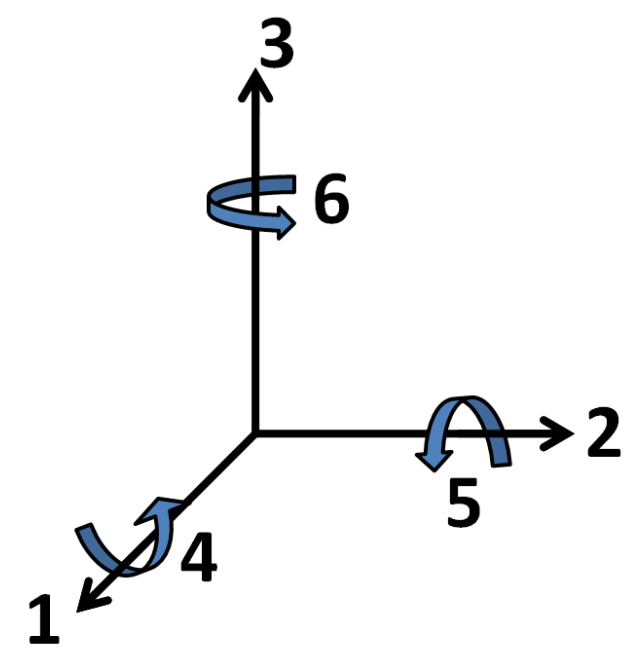

Figure 8 - The coordinate system for piezoelectric materials, where the 1, 2, and 3 axes are equivalent to the $x, y$, and $z$ axis, and the 4,5 , and 6 axes are shear forces.

Piezoelectric properties are often followed by two subscripts. For example, the piezoelectric charge coefficient, $d_{33}$, is followed by the number 33 . The piezoelectric charge coefficient is typically seen in units of picometers per volt $(\mathrm{pm} / \mathrm{V})$ and is used for actuation purposes since it tells you how far the piezoelectric will move per volt of electricity. Using the piezoelectric coordinate system, the first subscript signifies the direction of the applied electric field and the second subscript represents the direction of the displacement. This means that for the $d_{33}$, the electric field and the displacement are both in the 3 direction 
(Figure 9). In piezoelectric actuation, this mode is called the longitudinal or axial mode. There are 3 modes of piezoelectric actuation in total, (1) the axial mode, (2) the transverse mode, and (3) the shear mode.

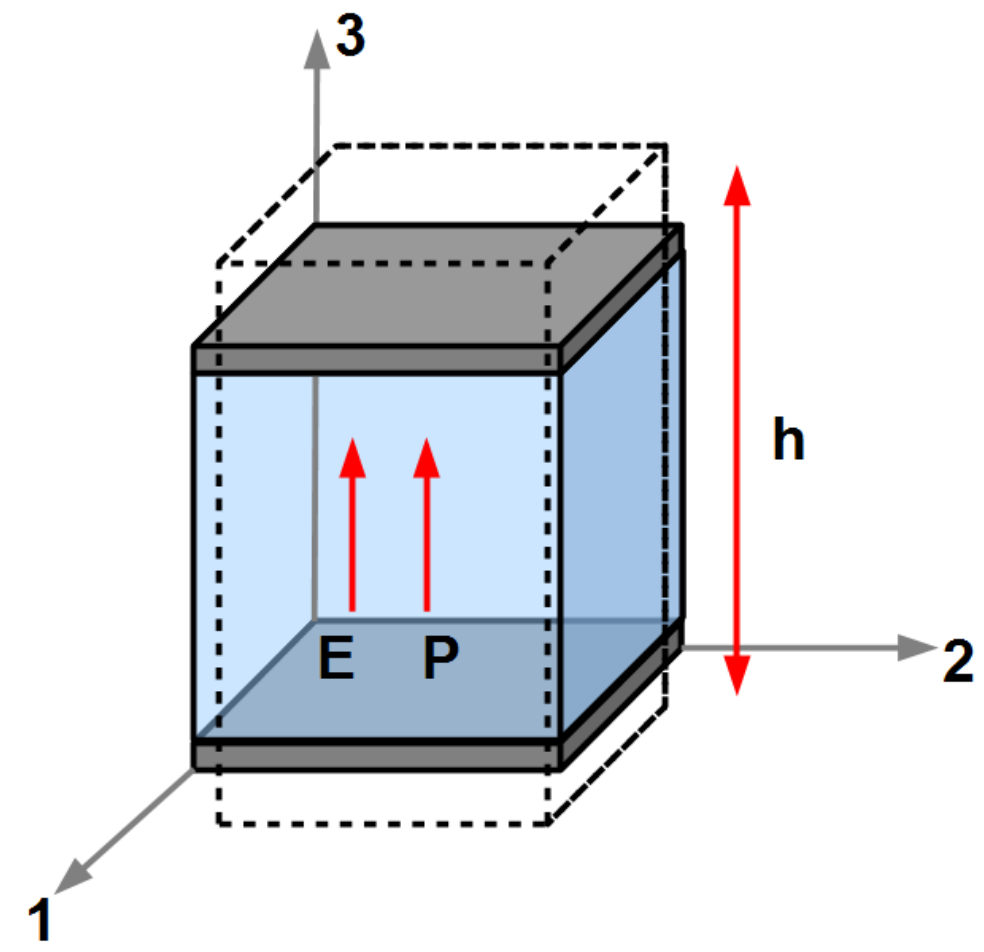

Figure 9 - The $d_{33}$ piezoelectric charge coefficient is used when the displacement of interest and the direction of the electric field are in the 3 direction.

Each mode uses a different piezoelectric charge coefficient. The axial mode uses the $d_{33}$, the transverse mode uses the $d_{31}$ (Figure 10), and the shear mode uses the $d_{15}$ piezoelectric charge coefficient (Figure 11). The axial mode is most often seen in layered piezoelectric stacks that are used to displace large distances. In this mode, the piezoelectric is polarized in what is called the thickness direction and is also expanding in the thickness direction. 


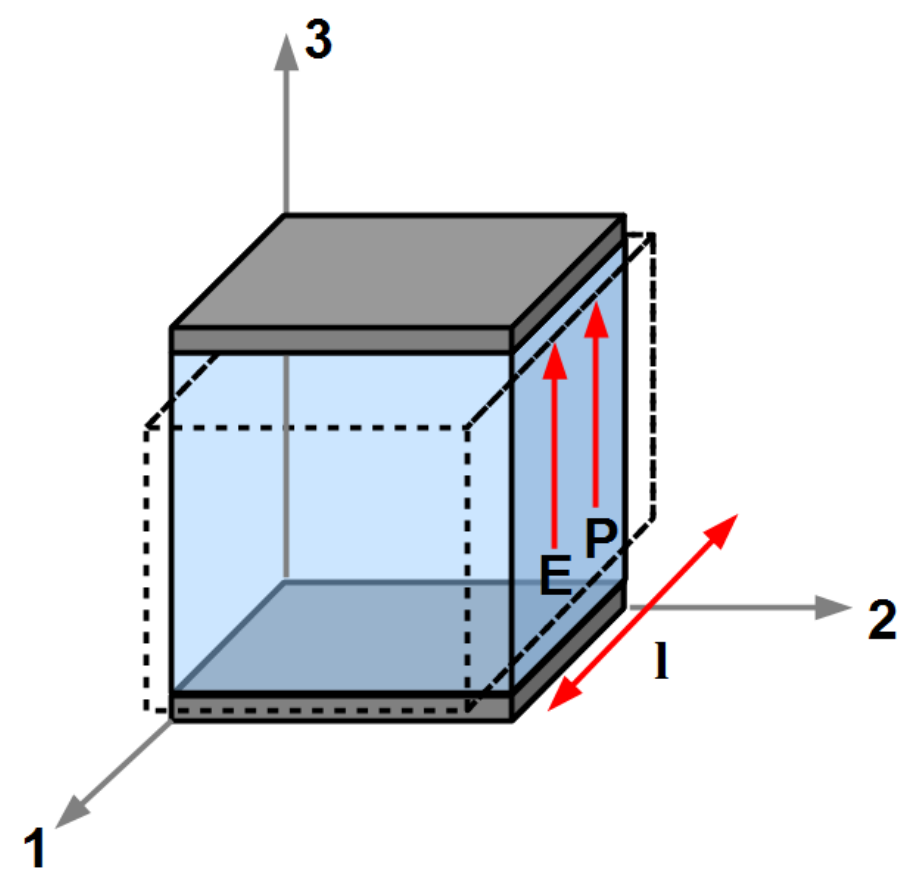

Figure 10 - In the transverse mode we use the $d_{31}$ charge coefficient because the electric field is applied through the 3 direction and the displacement of interest is in the 1 direction.

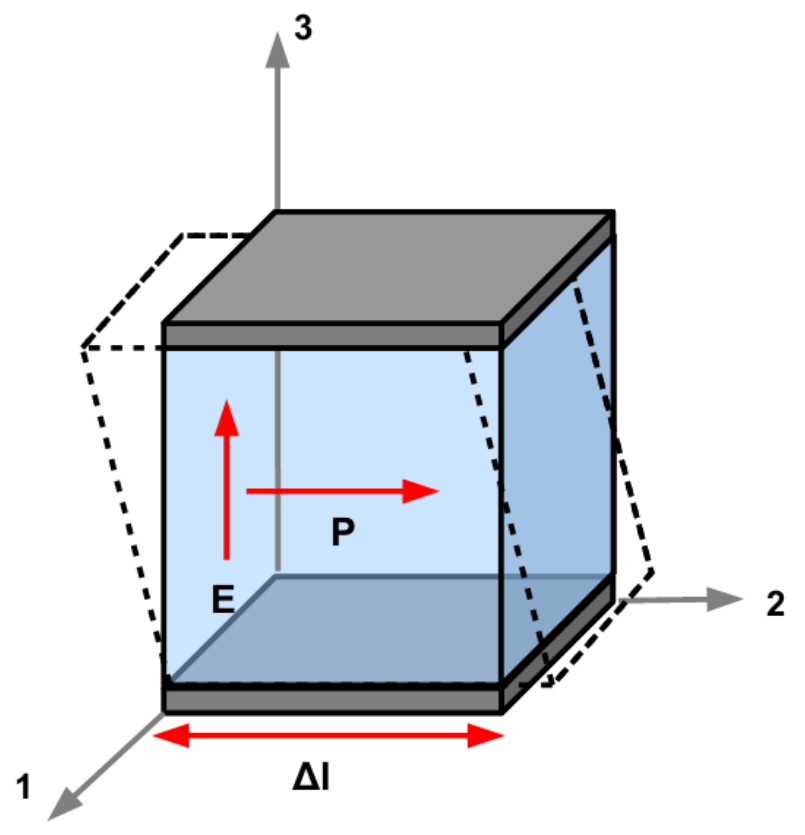

Figure 11 - When a piezoelectric is in the shear mode, the $d_{15}$ piezoelectric charge coefficient is used. This kind of device would be called a thickness-shear mode actuator.

The transverse mode is nearly the same as the axial mode because it has the same polarization and electric field direction. The only difference between the 
two is the displacement direction that we are interested in. An easy way to understand the differences is to take for example kneading bread dough (Figure 12).

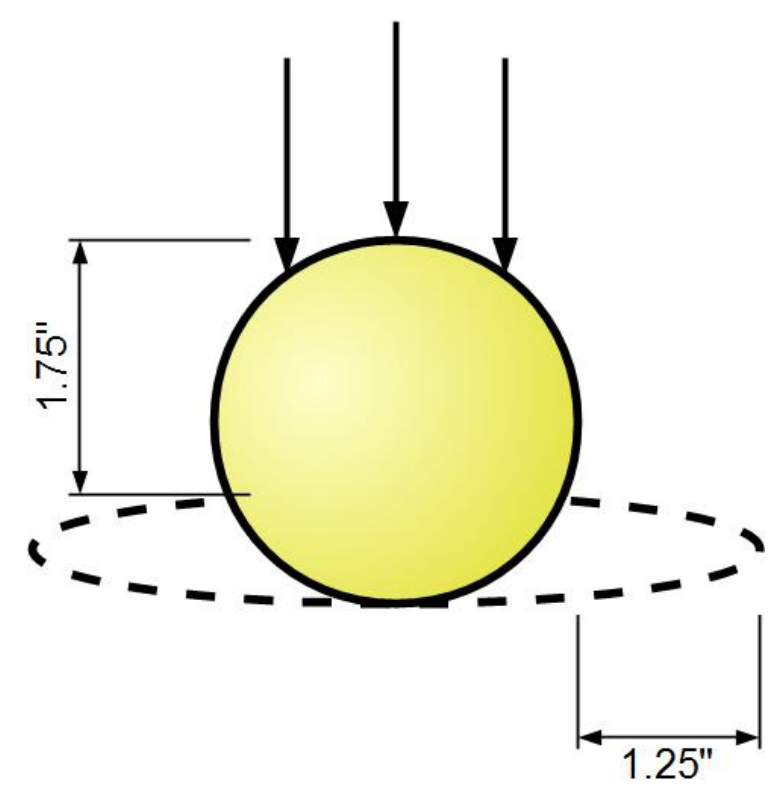

Figure 12 - When thinking of the transverse mode in piezoelectrics, think of when kneading bread dough. The dough will get smaller in the thickness direction, but will simultaneously get longer in the other direction.

In this example, a 2.5" ball of dough is kneaded and shrinks 1.75 inches in the thickness direction. Simultaneously, the dough also gets 1.25 inches longer in the other direction. If we are interested in how much the dough shrinks in the thickness direction (1.75 inches), then we talk about the axial mode $\left(d_{33}\right)$. When we want to know how much longer the dough gets (1.25 inches), then we use the transverse mode $\left(d_{31}\right)$. Despite the two different piezoelectric coefficients, the piezoelectric material has the same polarization and electric field direction. Due to the nature of piezoelectrics, a piezoelectric material will typically have a $d_{31}$ value that is negative and about half the value of the $d_{33 .}{ }^{5}$ The relationship between $d_{33}$ and $d_{15}$ is not as simple as that between $d_{33}$ and $d_{31}$ and will change depending on the type of piezoelectric material. For example, in the ferroelectric 
material, lead zirconate titanate (PZT), the $d_{15}$ is much higher than the $d_{33}$, but in zinc oxide the $d_{15}$ is smaller. If the displacement and applied voltage are known, then the following equation can be used to get an estimate of a piezoelectric material's $d_{33}$ (Equation 1). Where $d_{33}$ is the piezoelectric charge coefficient $[\mathrm{V} / \mathrm{m}], U$ is the applied voltage [V], and $\Delta L$ is the amount of displacement the piezoelectric experiences [m].

$$
d_{33}=\frac{\Delta h}{V}
$$

Equation 1 - To calculate an estimate of the piezoelectric charge coefficient, $d_{33}$, use the displacement and the applied voltage, which will result in units of $\mathrm{pm} / \mathrm{V}$.

In addition to the piezoelectric charge constants $\left(d_{33}, d_{31}, d_{15}\right)$, which are figures of merit for actuation, there are also the piezoelectric voltage constants, $g_{33}, g_{31}, g_{15}$, which are figures of merit for sensors. A figure of merit is a quantitative method of characterizing and comparing the performance of similar devices to each other. For the charge and voltage constants, the higher the figure of merit, the better the piezoelectric material is at actuating or sensing. The piezoelectric voltage constant, $g$, is the electric field that is generated per unit of mechanical stress that is applied to the piezoelectric material. Similar to the charge constants, the piezoelectric voltage constants also come with the subscripts 33,31 , and 15 . The first subscript is the direction of the generated electric field and the second subscript is the direction of the applied stress or force. Additional piezoelectric properties, electrical properties, and mechanical properties that are commonly used are listed and defined in the table below (Table I). For this study, I have found that the most important constant is the piezoelectric charge constant, $d_{33}$, because a higher $d_{33}$ value will result in a 
larger displacement of the microspeaker's diaphragm and a larger sound pressure. The biggest problem that I encountered was getting the diaphragm to oscillate enough to generate sound, although the issue was most likely a combination of insufficiencies such as displacement and force. In a microspeaker you want the polarization and displacement to be in the same direction because it will result in the most displacement and the greatest sound pressure level.

Table I - Common Piezoelectric, Electrical, and Physical Properties ${ }^{6}$

\begin{tabular}{|l|l|l|}
\hline \multicolumn{3}{|l|}{ Piezoelectric Properties } \\
\hline Variables & Units & Definition \\
\hline$d_{33}, d_{31}, d_{15}$ & $\mathrm{~m} / \mathrm{V}, \mathrm{C} / \mathrm{N}$ & Piezoelectric Charge Constants \\
\hline$g_{33}, g_{31}, g_{15}$ & $\mathrm{Vm} / \mathrm{N}$ & Piezoelectric Voltage Constants \\
\hline$k_{33}, k_{31}, k_{t}, k_{p}$ & & Electromechanical Coupling Factors \\
\hline Dielectric Properties & $\mathrm{F} / \mathrm{m}$ & $\begin{array}{l}\text { Permittivity, dielectric constants (constant } \\
\text { stress, constant strain) }\end{array}$ \\
\hline$\varepsilon^{\top}{ }_{11}, \varepsilon^{\mathrm{s}}{ }_{33}$ & & Relative dielectric constant (constant stress) \\
\hline $\mathrm{K}^{\top}$ & & Dielectric dissipation factor \\
\hline$\delta$, tan $\delta$ & & Quality factor \\
\hline $\mathrm{Q}$ & $\mathrm{N} / \mathrm{m}^{2}$ & Young's Modulus \\
\hline Physical Properties & $\mathrm{m}^{2} / \mathrm{N}$ & $\begin{array}{l}\text { Elastic Compliance (constant electric field, } \\
\text { constant displacement) }\end{array}$ \\
\hline $\mathrm{E}$ & \multicolumn{2}{|l}{} \\
\hline $\mathrm{s}^{\mathrm{E}}{ }_{11}, \mathrm{~s}^{\mathrm{D}}{ }_{33}$ &
\end{tabular}

The electromechanical coupling factors, $k_{33}, k_{31}, k_{t}$, and $k_{p}$, are a measure of a piezoelectric material's efficiency in converting mechanical energy into electrical energy or vice versa. The first subscript in $k$ indicates the direction of the electric field and the second subscript denotes the direction the mechanical force is applied. The electromechanical coupling factor, $k_{t}$, is used for discs or plates whose surface dimensions are small compared to their thickness. The planar coupling factor, $k_{p}$, is used for thin piezoelectric discs that experience radial 
coupling. The permittivity of a piezoelectric material, $\varepsilon$, is the resistance that is experienced when an electric field is formed in the medium or how an electric field affects or is affected by a piezoelectric material. $K$ is the relative dielectric constant and is the ratio between the amount of charge that the piezoelectric can store and the permittivity of free space, $\varepsilon_{0}$. The dissipation factor, $\tan \delta$, is a measure of the rate of energy loss in an oscillating piezoelectric system. It is typically measured with a capacitance bridge and is the reciprocal of $Q$, the quality factor. The quality factor, $Q$, is the quality of the oscillation of the system. A high $Q$ means that the system is oscillating efficiently and there is little energy loss over time. Young's Modulus, $E$, is a measure of a ceramic material's stiffness or elasticity. The elastic compliance, $s$, is the reciprocal of Young's Modulus and is followed by superscripts and subscripts. The superscript indicates whether the electric field $(E)$ or the electric displacement $(D)$ is constant. The first subscript in the elastic compliance indicates the direction of the strain and the second subscript indicates the direction of the stress. Despite this plethora of material properties, the most important one is still the piezoelectric charge coefficient, $d_{33}$. The other properties were not pursued in this study and are of minor importance for the fabrication of a working microspeaker. In different applications, these other properties may be of more importance to their operation.

\subsection{Zinc Oxide (ZnO)}

Zinc oxide $(\mathrm{ZnO})$ is the focus of this study and is an optically transparent piezoelectric material that is most often deposited using physical vapor 
deposition (PVD) and chemical vapor deposition (CVD) processes. It is also a semiconducting material with a wide direct bandgap of $\sim 3.3-3.4 \mathrm{eV}^{7}$ and a noncentrosymmetric hexagonal wurtzite crystal structure (Figure 13). ${ }^{8}$ Materials are considered to have wide band gaps if their bandgap energy is significantly greater than that of the common semiconductors like silicon $(1.1 \mathrm{eV})$ and gallium arsenide $(1.4 \mathrm{eV}) .{ }^{9}$ Wider bandgap materials like zinc oxide, silicon carbide, gallium nitride, and diamond are more efficient in high power and high temperature applications where the material limits of silicon based devices are being reached. ${ }^{10}$ They can withstand high powers because the electric breakdown voltage for wide bandgap materials tends to be much higher. $\mathrm{ZnO}$ is also a direct bandgap material that can undergo ultraviolet photoluminescence and can be used in UV light emitting applications. ${ }^{11}$ In a direct bandgap, the holes and electrons have the same momentum through the conduction and valence band, allowing the emission of photons from the electrons as they transition to lower energy states. However, in indirect bandgaps, such as in silicon, photons are not usually emitted because the electron has to transfer the momentum to the crystal lattice through an intermediate state. 

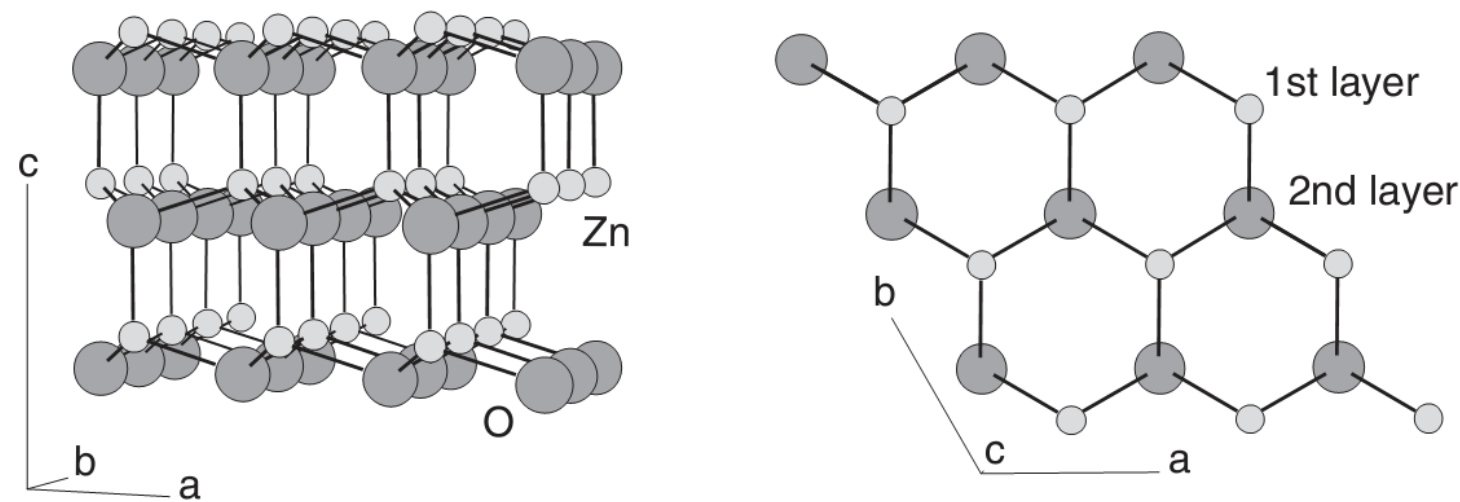

Figure 13 - Zinc oxide has a hexagonal wurtzite crystal structure that is non-centrosymmetric, which gives $\mathrm{ZnO}$ its unique piezoelectric properties. ${ }^{7}$

When deposited, this piezoelectric material is polycrystalline and the crystals typically form a columnar structure and must be similarly distributed and oriented in the c-axis direction to exhibit piezoelectric behavior. ZnO's piezoelectric properties are quantitatively characterized with several different piezoelectric coefficients, the most important of which is called the $d_{33}$, or the piezoelectric charge constant (Table II). ZnO has a $d_{33}$ piezoelectric coefficient that can vary depending on the quality of the film and the orientation of the crystals, but it is often reported as $12.4 \mathrm{pm} / \mathrm{V}$, which means that for every volt that is applied it will move only 12.4 picometers. $^{12}$ 
Table II - Material Properties of ZnO

\begin{tabular}{|c|c|c|}
\hline Parameter & Value & Units \\
\hline Density & 5.675 & $10^{3} \mathrm{~kg} / \mathrm{m}^{3}$ \\
\hline$\varepsilon_{11} / \varepsilon_{0}$ & 8.5 & dimensionless \\
$\varepsilon_{33} / \varepsilon_{0}$ & 10.9 & \\
\hline$d_{33}$ & 12.4 & \\
$d_{31}$ & -5 & $10^{12} \mathrm{pm} / \mathrm{V}$ \\
$d_{15}$ & -8.3 & \\
\hline$e_{33}$ & 1.32 & \\
$e_{31}$ & -0.57 & $\mathrm{C} / \mathrm{m}^{2}$ \\
$e_{15}$ & -0.48 & \\
\hline$c_{11}$ & 210 & \\
$c_{12}$ & 121 & \\
$c_{13}$ & 105 & $10^{9} \mathrm{~N} / \mathrm{m}^{2}$ \\
$c_{33}$ & 211 & \\
$c_{44}$ & 43 & \\
\hline
\end{tabular}

For comparison, lead zirconate titanate (PZT), one of the most common types of piezoelectric materials, has one of the highest $d_{33}$ values of about $500 \mathrm{pm} / \mathrm{V}$. A low piezoelectric charge constant, $d_{33}$, correlates to a weak piezoelectric response and small amounts of displacement, but will tend to have other benefits such as a much higher resonance frequency and quality factor, $\mathrm{Q}$. ZnO is typically used for high frequency applications such as surface acoustic wave (SAW) devices, microspeakers, and highly sensitive sensors. PZT is more useful for micropositioning devices like in piezoelectric stacks, which require lots of displacement. Unlike PZT, ZnO is not usually used for micropositioning purposes because of its small displacements.

$\mathrm{ZnO}$ is becoming a much more popular material because it's starting to replace other piezoelectrics and can even replace other transparent conductive electrodes like indium tin oxide (ITO) that are becoming increasingly expensive. $\mathrm{ZnO}$ is commonly used in microfabrication because it is simple and easy to deposit and can be deposited in a variety of different ways that are commonly 
available such as chemical vapor deposition, physical vapor deposition, pulsed laser deposition, and thermal evaporation. Another allure of $\mathrm{ZnO}$ is that it is biocompatible and contains no lead, unlike other common piezoelectric materials PZT and barium titanate $\left(\mathrm{BaTiO}_{3}\right)$. One of the big differences between $\mathrm{ZnO}$ and other powder based piezoelectric ceramics like PZT, is that it has an extremely low piezoelectric coefficient, which is the number that piezoelectric materials are typically measured with.

\subsubsection{Deposition Techniques}

$\mathrm{ZnO}$ can be deposited using a number of different deposition techniques, such as physical vapor deposition, chemical vapor deposition, pulsed-laser deposition, molecular beam epitaxy, spray-pyrolysis, and electro-chemical deposition. ${ }^{7}$ Physical vapor deposition (PVD), which is also known as sputtering, is what will be utilized because sputtering $\mathrm{ZnO}$ is well-documented and is possible with the equipment in the Cal Poly Microfabrication laboratory.

\subsubsection{DC Sputtering}

DC sputtering, which is a form of physical vapor deposition, originates from a momentum transfer of fast moving ions colliding with a cathode or target material. Essentially, the fast ions hit the surface of the target and cause ejection of the topmost atoms of the target. ${ }^{13} \mathrm{DC}$ sputtering begins with the ignition of a glow discharge or plasma through a large DC bias between a cathode and an

anode under a vacuum (Figure 14) $\cdot{ }^{16}$ In this case, the cathode of the system is a pure zinc target and the anode is the chamber of the vacuum system. When a vacuum has been achieved, high purity $\mathrm{Ar}$ and $\mathrm{O}_{2}$ gas are pumped into the 
chamber in desired ratios. This causes the target to oxidize into $\mathrm{ZnO}$ and when the argon and oxygen ions collide with the target they will sputter a $\mathrm{ZnO}$ thin film onto the substrate's surface.

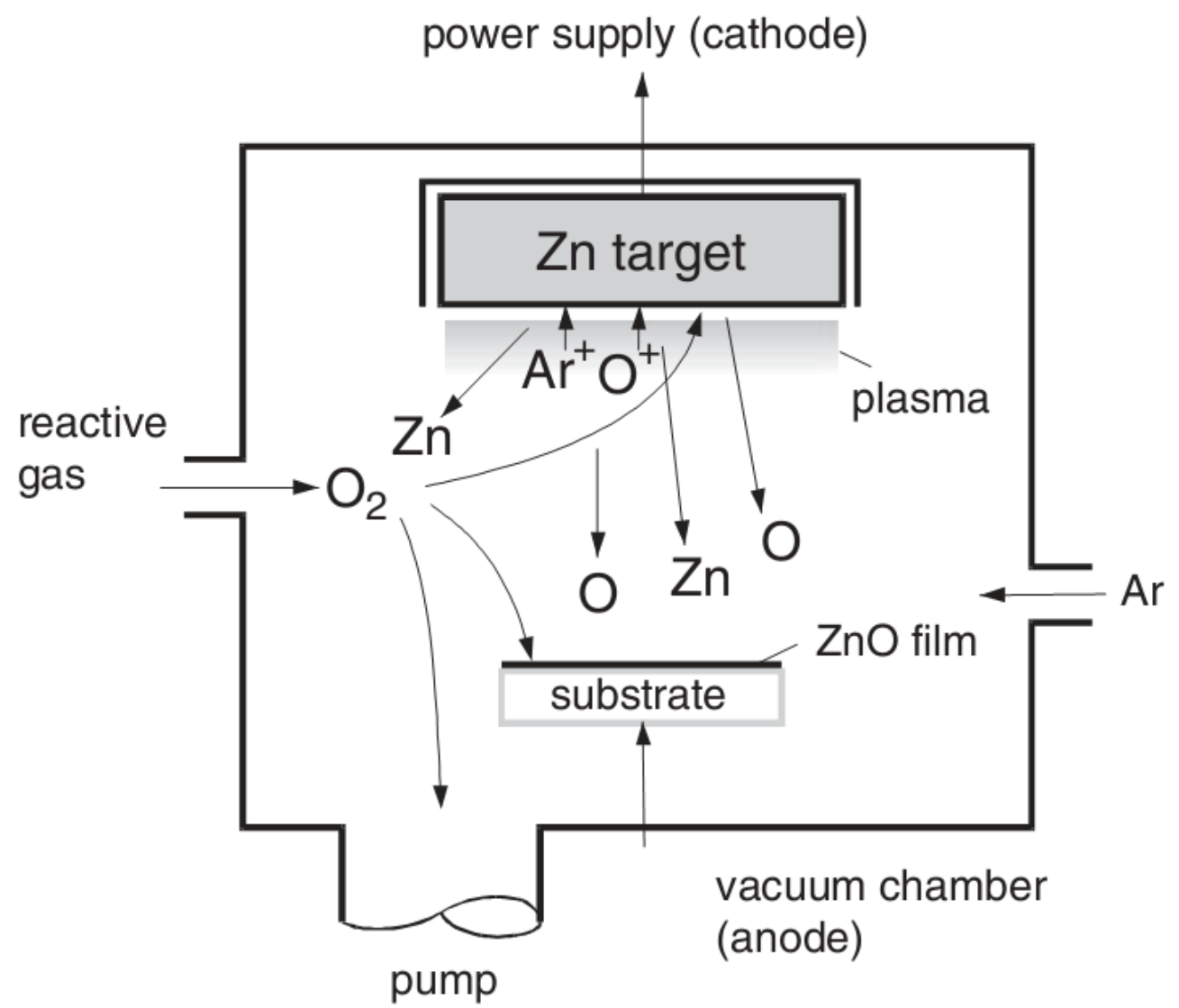

Figure 14 - The $\mathrm{ZnO}$ thin film is deposited using physical vapor deposition (PVD), or sputtering. Sputtering occurs when ionized argon and oxygen gas collide with the surface or the zinc target, ejecting zinc atoms that react with the oxygen gas in the chamber and deposit onto the surface of the substrate. ${ }^{10}$

The collision of ions with the target's surface can cause a number of events to occur, such as ion implantation, ion reflection, the ejection of secondary electrons, as well as the ejection of the target's atoms (Figure 15). ${ }^{13}$ These reactions can cause intense heating of the target material and therefore the target must be cooled, typically by water. During sputtering, the substrate is also affected by this ion bombardment, which can cause a number of reactions to 
occur, such as heating of the substrate up to temperatures around $150^{\circ} \mathrm{C} .{ }^{14}$ This increased temperature is actually beneficial for the resulting film because the surface mobility of the atoms is enhanced. If the bombarding particle's energy is high enough, it can either implant itself into the film, damaging the film, or it can transfer its momentum to a surface atom, causing it to resputter. ${ }^{15} \mathrm{~A}$ common side effect of sputtering $\mathrm{ZnO}$ is the creation of voids in the film.

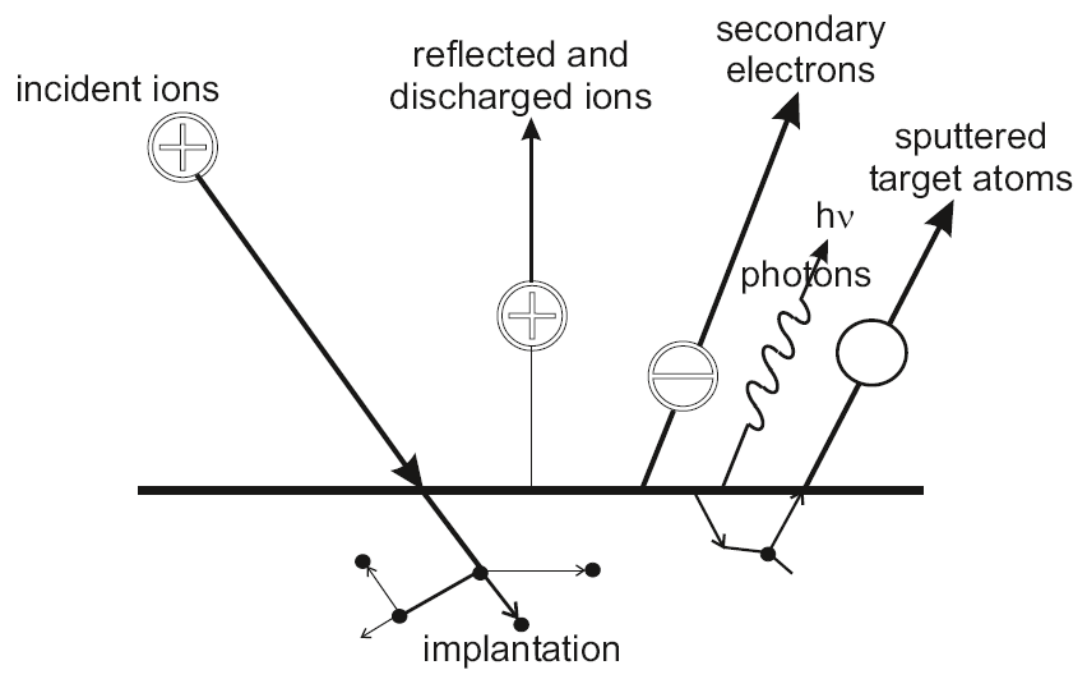

Figure 15 - During sputtering, incident ions collide with the surface of the target material, which causes several cascading events to occur, including ion implantation, ejected target atoms, and the release of secondary electrons. ${ }^{13}$

The two methods that are commonly used for physical vapor deposition are radio-frequency $(\mathrm{RF})$ sputtering and direct current (DC) sputtering. These PVD techniques are often accompanied by what is called magnetron sputtering. Magnetron sputtering is a type of sputtering that uses magnets to confine the electrons around a certain location. The magnets are usually placed behind the cathode and keep the plasma close to the target, allowing more collisions to occur and thus resulting in a faster sputtering rate (Figure 16). ${ }^{16}$ Unfortunately, magnetron sputtering is often wasteful and cannot utilize the entirety of a 
sputtering target because of the racetrack. Only a circular racetrack of the target is deposited and the rest is not utilized. However, magnetron sputtering also allows sputtering to occur at lower pressures, which often improves the quality of the deposited thin films.

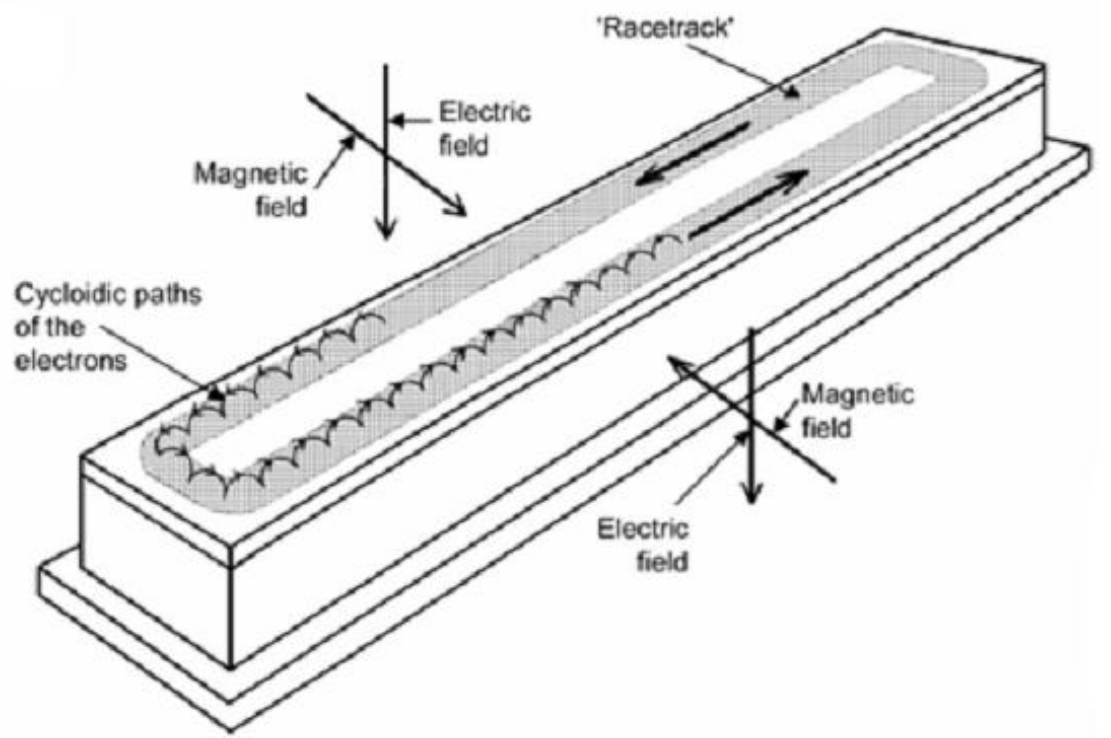

Figure 16 - Magnetron sputtering uses magnetic fields to confine the plasma close to the cathode in what is called the racetrack. This increases the sputter rate and allows for sputtering at lower pressures. ${ }^{16}$

DC sputtering techniques can only sputter conductive materials and cannot sputter ceramic targets because their insulating properties causes positive charge to build up on the target, which halts the sputtering process.

Sputtering and characterizing $\mathrm{ZnO}$ thin films is the first step to being able to utilize piezoelectrics in microelectromechanical systems (MEMS). A piezoelectric material has never been produced in the Materials Engineering department at Cal Poly and being able to create a high quality piezoelectric $\mathrm{ZnO}$ thin film should help the fabrication of a working microspeaker MEMS device. 


\subsubsection{Reactive Sputtering}

Reactive sputtering is extremely similar to DC sputtering or RF sputtering except instead of a single gas being injected into the vacuum chamber, another reactive gas is introduced (Figure 14). For sputtering $\mathrm{ZnO}$, the reactive gas is an ultra-high purity oxygen gas. The oxygen gas is called a reactive gas because it is not a noble gas and will undergo chemical reactions to gain electrons. When the oxygen is introduced into the chamber, the zinc atoms that are ejected from the zinc target by the argon ions react with oxygen atoms and form zinc oxide. DC reactive sputtering was performed in this project because DC reactively sputtered $\mathrm{ZnO}$ thin films generally result in films with larger grain sizes and higher piezoelectric coefficients. ${ }^{14}$

\subsubsection{RF Sputtering}

$\mathrm{RF}$ sputtering is when a high frequency alternating signal is applied to a metal plate with an insulating target attached. The metal plate and insulating target are placed within a low-pressure plasma discharge, which causes positive charge to accumulate on the target and neutralize during half of every cycle (Figure 17). ${ }^{17}$ A RF driven sputtering has more efficient ion generation than DC sputtering and therefore results in higher sputtering rates. ${ }^{13}$ However, as a result of RF sputtering's high deposition rate, the grains and crystals do not have time for growth and nucleation, resulting in extremely small crystal diameters. Schuler compared the properties of DC sputtered and RF sputtered $\mathrm{ZnO}$ and reported that DC sputtered grain sizes were around $200 \mathrm{~nm}$ and RF sputtered grains were around $50 \mathrm{~nm} \cdot{ }^{18}$ Schuler also found that the surface roughnesses of DC 
sputtered $\mathrm{ZnO}$ thin films were significantly greater than RF sputtered ones.

These small crystal sizes can sometimes result in RF sputtered $\mathrm{ZnO}$ thin films

with little to no piezoelectric properties. ${ }^{18}$ Also, reactive sputtering is not generally utilized in RF sputtering techniques.

RF sputtering enables the sputtering of non-conductive targets because it can avoid charge build up on the target because the sign of bias at the anode and cathode is switched at high rates. ${ }^{17} \mathrm{RF}$ sputtering of $\mathrm{ZnO}$ thin films typically involves a high purity ceramic $\mathrm{ZnO}$ target that is used in conjunction with an $\mathrm{Ar}$ sputtering gas. ${ }^{18}$

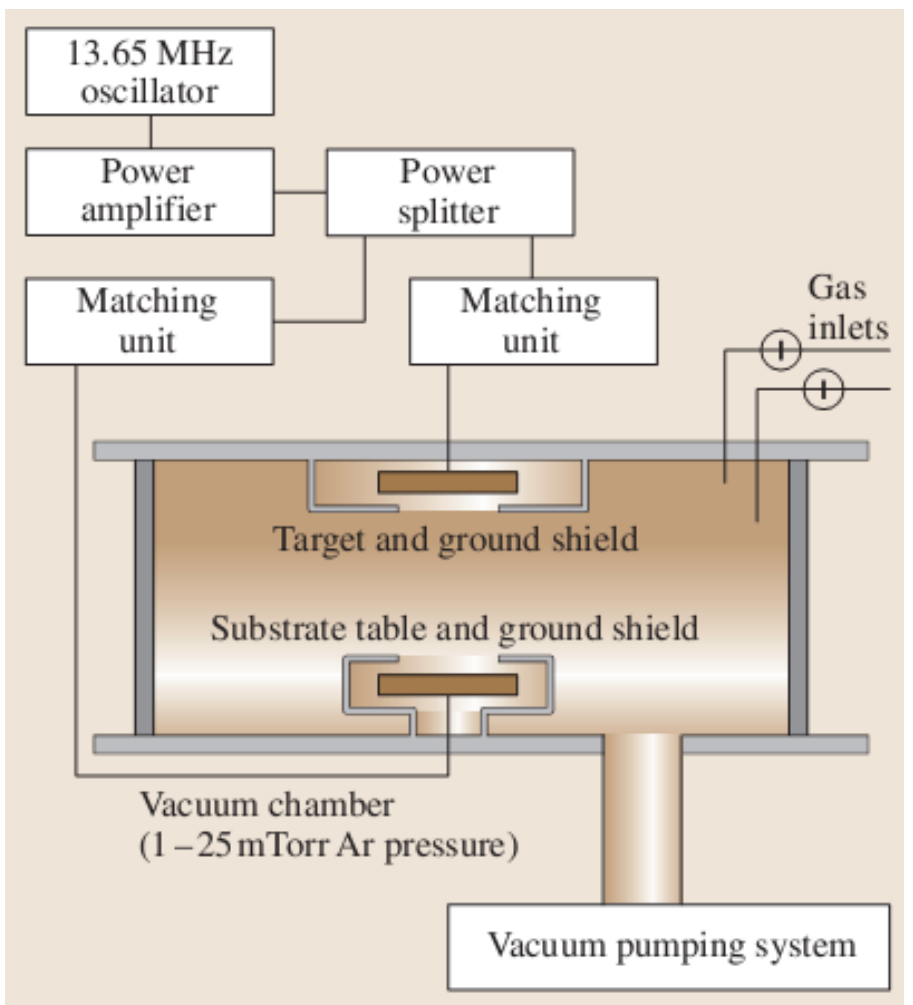

Figure 17 - A typical RF sputtering system that can sputter both non-conductive and conductive materials because of its high frequency alternating signal provided by an oscillator. 


\subsection{Characterization Techniques}

After attempting to fabricate a microspeaker with little characterization of the deposited ZnO films I experienced little success. I realized that I needed to know more about the deposited thin films before I could fabricate a working device. $\mathrm{ZnO}$ characterization is typically performed by looking at the thin film's structural properties, optical properties, electrical properties, and piezoelectric properties. Once these properties are better known, I will know the correct sputtering parameters to obtain the best properties for a microspeaker.

\subsubsection{Structural Properties}

The simplest method of characterization is to determine ZnO's structural properties. With structural characterization, I will be able to find out the size of the crystals, the thickness of the films, and the physical structure and shape of the crystals. Highly piezoelectric $\mathrm{ZnO}$ thin films typically have a columnar structure and therefore cross-sectional imaging needs to be performed. With crosssectional SEM imaging and profilometer scans, I will be able to find the thickness of the films. The SEM images will also be able to verify the profilometer's results. Topographical images of the $\mathrm{ZnO}$ surface will also be taken in order to find out the size and shape of the crystals. Scanning electron microscopy (SEM) is the best method of obtaining these micrographs.

\subsubsection{Scanning Electron Microscopy (SEM)}

Unlike in optical microscopy, where photons from a light source are focused through a series of lenses, scanning electron microscopy uses the emission of electrons to see a specimen. A SEM can determine the topography of an object 
by quickly scanning the object's surface with an electron beam in a raster pattern under an ultra high vacuum. The electrons penetrate and interact with the surface layer of atoms in the specimen and produce signals that the electron detector can interpret and deliver to the computer to convert into visual signals. Typically there are three different signals that are received from the specimen when hit with the electron beam, secondary electrons, backscattered electrons, and x-rays. There are also other types of signals that can be used to determine the properties of the specimen, such as Auger electrons, cathodluminescence, transmitted electrons, and specimen current. When the electron beam hits the surface of the specimen, the electrons will penetrate the surface in what is called the "interaction volume" (Figure 18). ${ }^{19}$

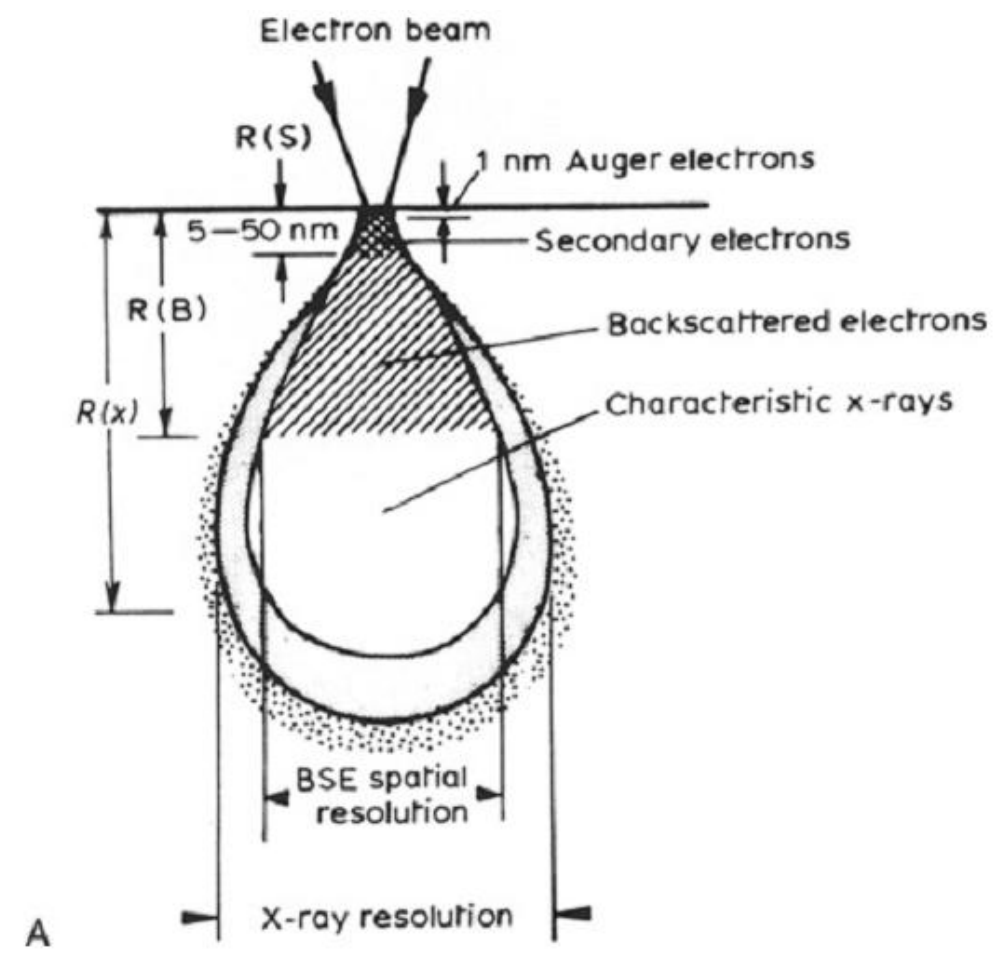

Figure 18 - When the electron beam hits the specimen's surface, secondary electrons, backscattered electrons, and characteristic x-rays are emitted from the specimen. ${ }^{19}$ 
SEMs can have several detectors in the specimen chamber, typically a secondary electron detector, a backscattered electron detector, and an energydispersive x-ray spectrometer (EDS) or wavelength-dispersive x-ray spectrometer (WDS). A vacuum system, which typically consists of a mechanical pump and a diffusion pump, keeps the electron gun contaminant free and the electron beam from scattering off of other atoms in the chamber. When the electron beam hits the specimen, secondary electrons are emitted from 5-50 nm below the surface with low energies around $50 \mathrm{eV}$. These secondary electrons are emitted into the vacuum chamber and are detected with the secondary electron detector. Because the secondary electrons are emitted from the very top of the surface, they only can give topographical information about the specimen. In addition to the object's topography, a SEM can also determine the structural features deeper under the surface. To get this sub-surface information about the specimen, higher energy backscattered electrons are required. If $E_{0}$ is the energy of the incident electron beam (which can often vary from 1-20 keV) and $E_{B S E}$ is the energy of the backscattered electrons, then $50 \mathrm{eV}<E_{B S E}<E_{0 .}{ }^{20}$ These backscattered electrons experience elastic collisions with the specimen and therefore have much more energy than secondary electrons. This means that the backscattered electrons can come from much deeper within the interaction volume and can give more information about the specimen's underlying structure, such as grain boundaries, phase boundaries, composition, etc. In addition to backscattered electrons, characteristic $\mathrm{x}$-rays are also produced and can be used to determine the composition of the specimen. With the energy- 
dispersive x-ray spectrometer (EDS), the SEM can detect the discrete energy levels of the emitted characteristic $x$-rays. The EDS will typically result in a spectrum of intensity over energy, where each element has a specific energy level that can be classified. The power of the electron beam has no effect on the position of these discrete energy levels and thus, these energy levels can be used to determine the composition of the specimen. ${ }^{19}$

SEM imaging typically begins with the thermionic emission of electrons from a tungsten filament (Figure 19). The electron beam is aimed towards the specimen through an aperture and its size and shape are changed by magnets, similar to how optical lenses change the shape and size of the light in an optical microscope. Magnetic fields or electrostatics can focus the electron beam, but magnetic fields produce smaller aberrations than electrostatics, although both handle aberrations poorly compared to glass lenses. ${ }^{21}$ The magnetic fields that adjust the electron trajectories are produced with coils of wire that are known as electromagnets and the magnetic field can be adjusted by the current applied to the coils. When the current is adjusted, it will change the position of the focal point of the beam. 


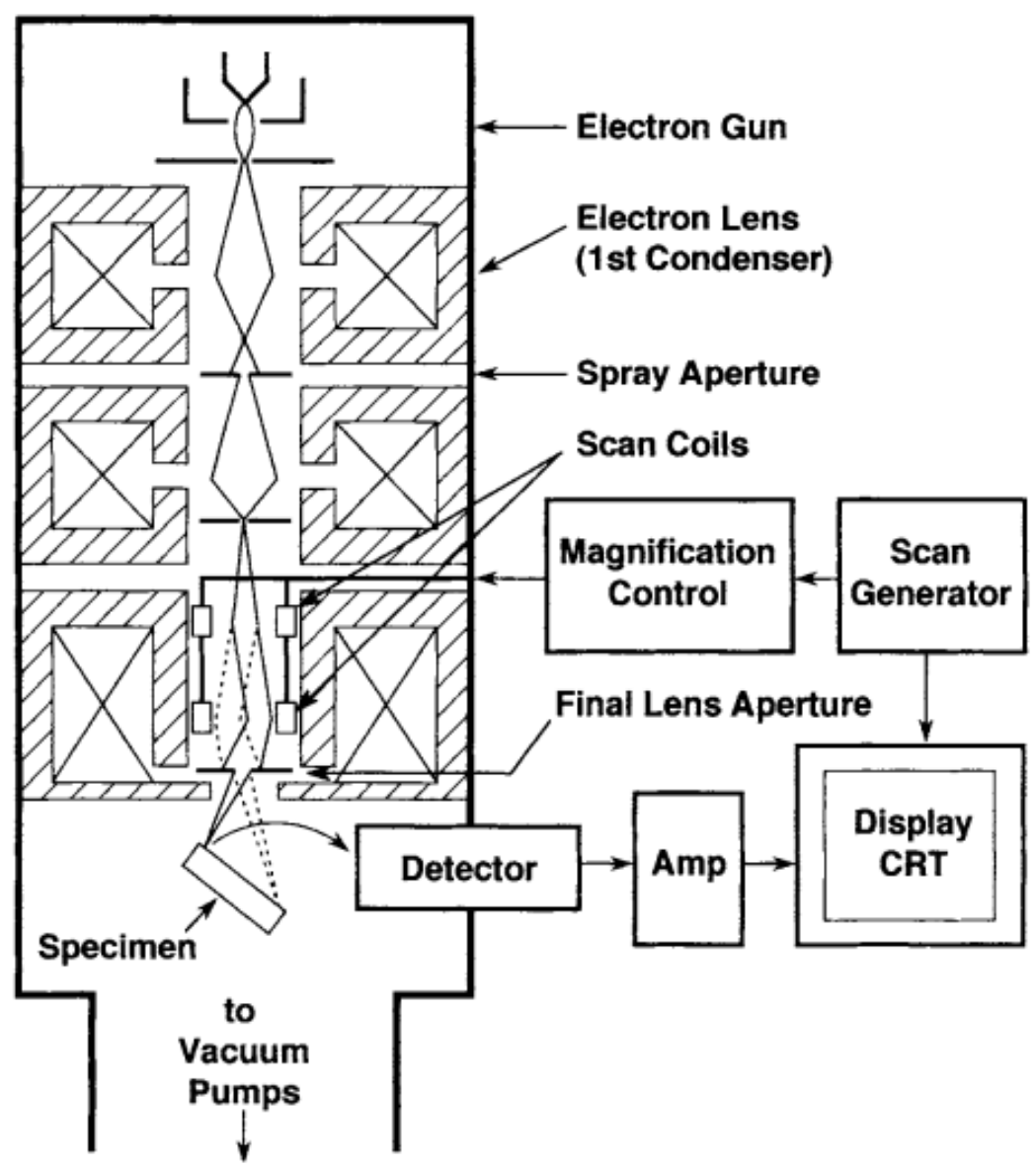

Figure 19 - A schematic of an SEM. The essential piece of an SEM is the electron beam that is thermionically emitted from a tungsten filament and is then condensed and focused onto the specimen using electromagnetic lenses. ${ }^{19}$

The first lens that the electron beam goes through is the condenser lens. The condenser lens takes the wide beam coming out of the aperture and condenses and collimates it. In modern electron microscopes, the beam is sometimes sent through a second condenser lens in order to provide more control over the beam. After the condenser lenses, the beam is sent through objective lenses that focus the beam into a fine point at the specimen surface and supply demagnification. After the objective lens, the beam goes through a final lens aperture called the "real" aperture. The apertures before this final aperture are referred to as virtual apertures and perform the same as the real aperture by excluding scattered 
electrons. The real aperture affects the beam shape and the beam edge sharpness and can be altered by the SEM operator using spot size, which adjusts the aperture size and enhances the image resolution and depth of field. ${ }^{21}$ The cross-sectional shape of the final beam can be affected by lens defects from machining, in the winding of the coils, or contamination of the apertures. The defects typically cause the beam shape to become elliptical instead of circular and can cause distortion and stretching in the final image when in-focus, overfocused, or underfocused (Figure 20). ${ }^{21}$ A stigmator, which is a series of coils that surrounds the electron beam, can correct for astigmatism and improve the resolution of the image.

(a)

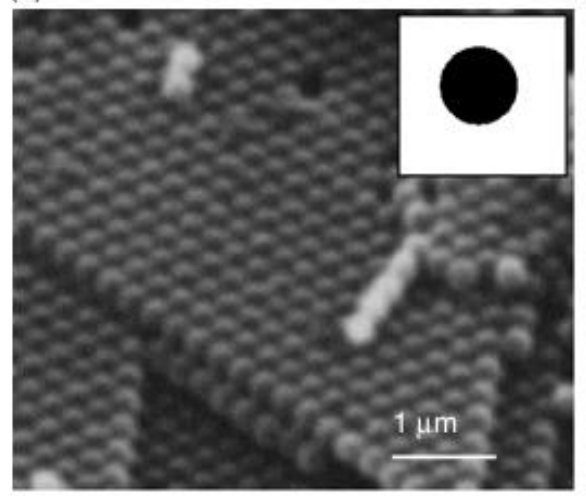

(c)

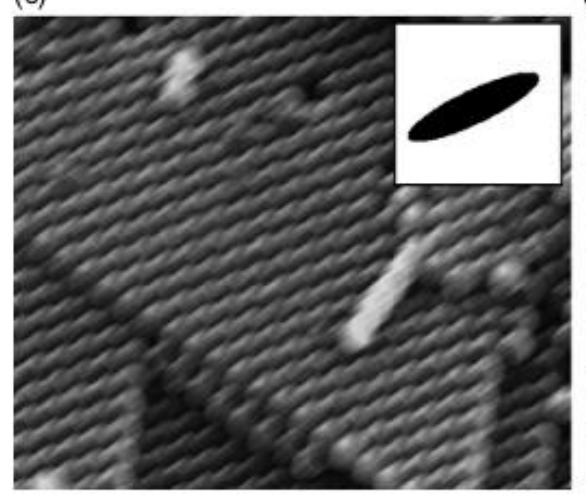

(b)

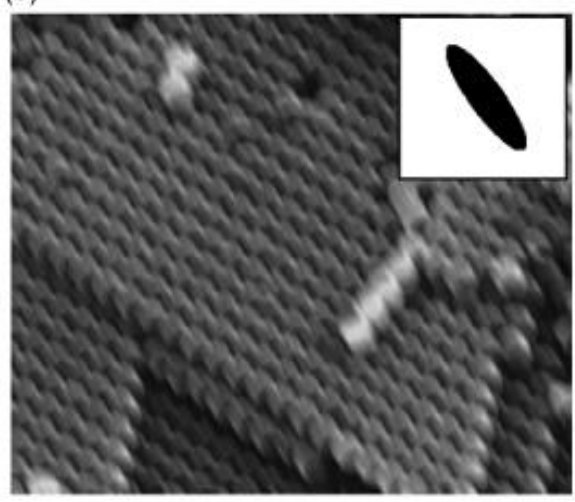

(d)

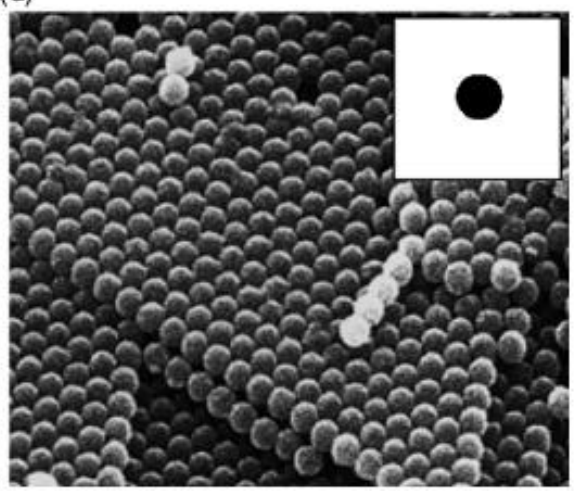

Figure 20 - SEM images of an opal structure with astigmatism in the a) in-focus condition, b) underfocused condition, c) over-focused condition, and d) in-focus condition without astigmatism. ${ }^{21}$ 
The working distance, or WD, is the distance between the specimen and the real aperture and can affect the depth of field in the image and the resolution of the image. A higher WD results in more depth of field, which makes more of the image in focus. A large WD is useful when taking images of samples with lots of height variation in their topography. When depth of field is not important because the specimen's surface is flat, a smaller WD should be used because higher resolutions can be achieved. ${ }^{21}$

There are other types of filaments that are sometimes used, such as lanthanum hexaboride $\left(\mathrm{LaB}_{6}\right)$, but they typically require a better vacuum to operate in a stable and reliable manner. ${ }^{19}$ The better vacuum is required because $\mathrm{LaB}_{6}$ burns at a lower temperature than tungsten. The high temperature of the tungsten makes it a self-cleaning device that can burn off residual gases from the vacuum system that can poison the filament. ${ }^{19}$ The $\mathrm{LaB}_{6}$ is not self-cleaning and therefore requires a better vacuum to prevent emitter poisoning. A tungsten filament is used because tungsten is relatively inexpensive, has an extremely high melting temperature, and a low vapor pressure.

\subsubsection{X-Ray Diffraction (XRD)}

$\mathrm{X}$-ray diffraction $(\mathrm{XRD})$ is a material characterization technique that is used to determine the crystalline quality, chemical composition, and atomic structure of a solid material. X-rays are typically generated by accelerating electrons at a metal target at several $\mathrm{keV}$, which causes the core electrons in the metal target to become knocked out by the energized electrons. When the higher shell electrons fall into the vacancies in the core shell, it causes x-rays to be emitted. A copper 
source is one of the most common metal targets. These $\mathrm{x}$-rays are called characteristic $\mathrm{x}$-rays because they have well-defined wavelengths that correspond to the energy difference between the two shells in the metal target. ${ }^{22}$ The characteristic x-rays are sent towards the sample and cause the atoms and their electrons to scatter the x-rays (Figure 21).

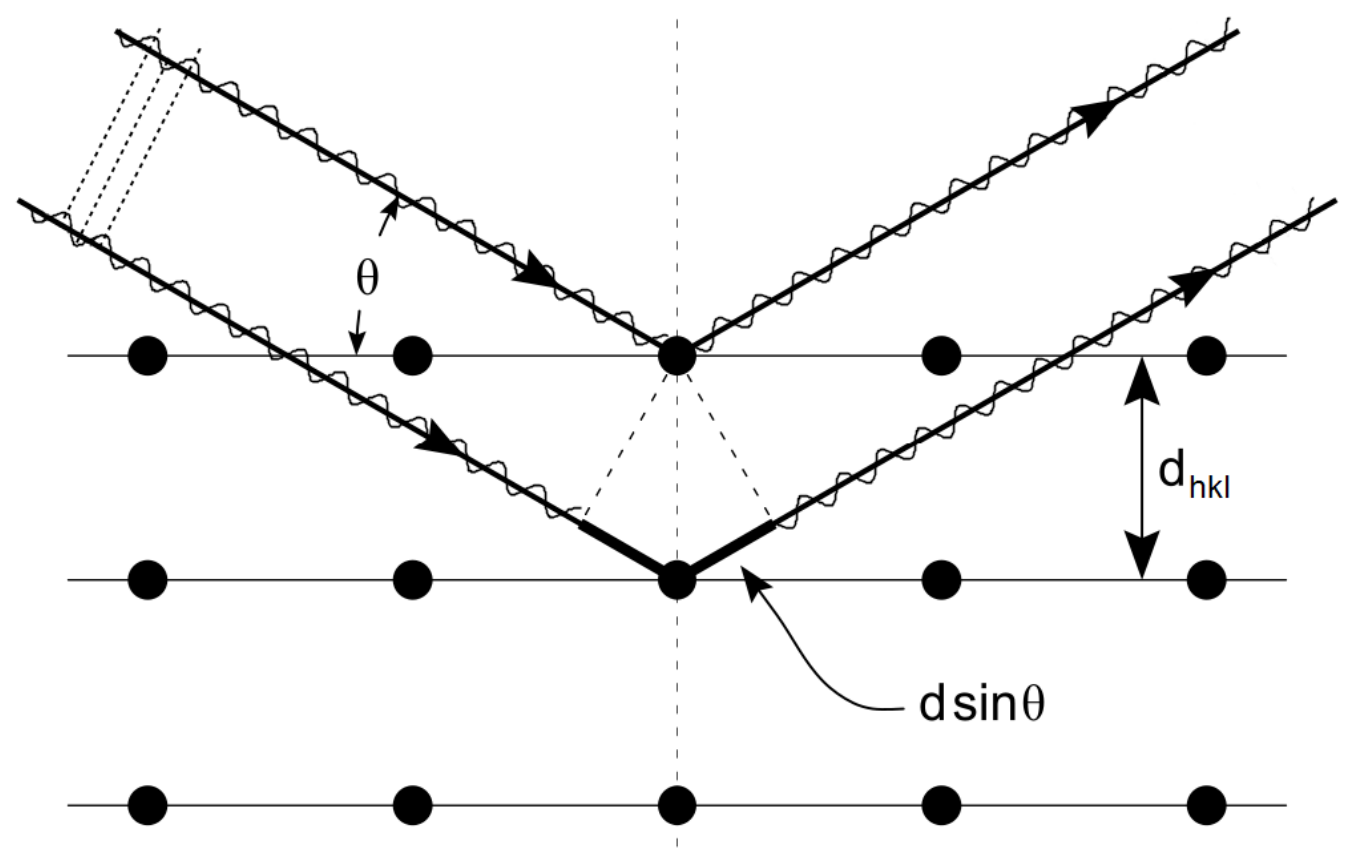

Figure 21 - When incident $x$-rays penetrate the surface of a sample the electrons in the atoms scatter the $x$ ray waves and send out new waves that can constructively interfere with one another in certain directions according to Bragg's law.

Since the wavelengths of the $x$-rays $(1-100 \AA)$ are similar in size to the interatomic spacing $\left(d_{h k l}\right)$ of the material, the waves diffract and scatter, gathering and carrying information about the material's individual atoms and their arrangement. Crystalline samples, which possess long-range order in their atomic arrangement represented by crystal structures, are easy to measure because they scatter strongly in certain directions. The building block of the crystalline sample is the crystal structure and its unit cell. According to Bragg's law, these unit cells will all scatter the $\mathrm{x}$-rays in the same direction and if the path-length difference 
$2 d_{h k l} \sin \theta$ equals an integer multiple of the wavelength $n \lambda$, then constructive interference occurs (Equation 2). ${ }^{23}$

$$
n \lambda=2 d_{h k l} \sin \theta
$$

Equation 2 - Bragg's law describes at which angles and wavelengths an incident $x$-ray wave will cause constructive interference of the scattered waves. ${ }^{23}$

Where $n$ is an integer, $\lambda$ is the wavelength of the incident wave, $d_{h k l}$ is the spacing between the planes in the atomic lattice, and $\theta$ is the angle between the scattered wave and the atomic plane. X-rays scattered in other directions will experience destructive interference and will be out of phase with each other. A diffraction pattern is obtained by measuring the intensity of the scattered waves at across a number of incident x-ray angles (Figure 22).

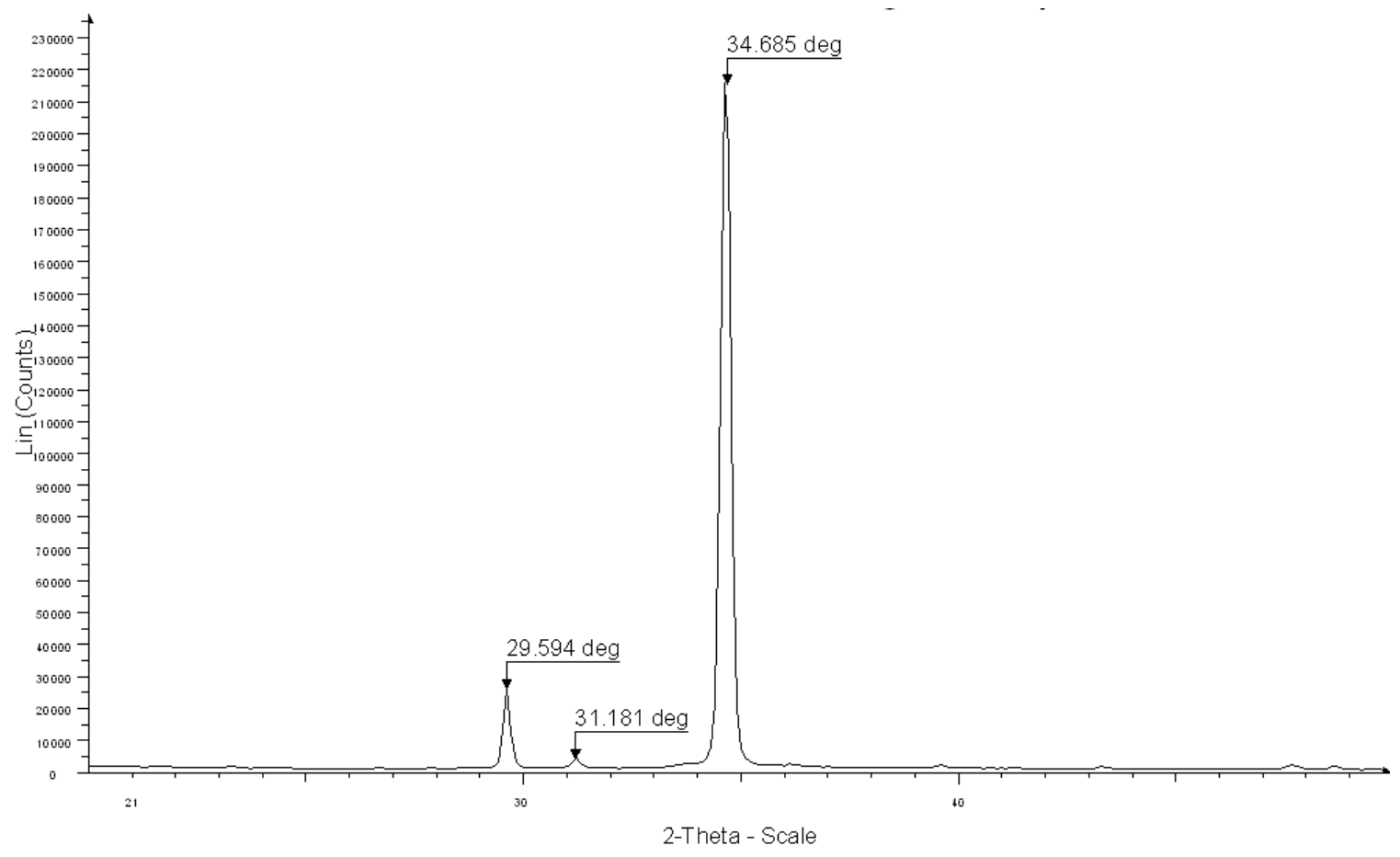

Figure 22 - The diffraction pattern for an annealed $\mathrm{ZnO}$ sample deposited on silicon. The distinctive Bragg's peak at $34.685^{\circ}$ indicates the (002) crystal plane, which also indicates that the $\mathrm{ZnO}$ crystals are c-axis oriented.

Bragg peaks are formed when the Bragg condition of constructive interference is satisfied. The intensity of the Bragg peaks is determined by the 
atomic arrangement in the unit cell. One of the parameters that the XRD can calculate is the full-width at half-maximum (FWHM), which can be used to measure a material's crystalline quality. ${ }^{24} \mathrm{~A}$ high FWHM value describes a Bragg's peak that is wide and broad, which indicates poor crystallinity and small crystallite size..$^{25}$

\subsubsection{Stylus Profilometry}

Stylus profilometry is a characterization technique that is used to determine properties like a film's thicknesses, surface roughness, or radius of curvature. A stylus profilometer uses a diamond coated stainless steel tip that is dragged across the surface of the specimen a specific distance with a specific contact force. There are several different configurations for a stylus profilometer to receive its signals. In one configuration, a laser is bounced off of the tip of the cantilever into a quad photodiode that will detect the changes in height through the changes in the voltages from the photodiode. Another configuration drags the stylus over the surface, which is sensed by a pick-up and then sent to a piezoelectric or inductive transducer that converts the movement into an electrical signal (Figure 23). ${ }^{26}$ The signal is amplified, digitized, filtered, and then it results in calculated parameters and a surface profile. 


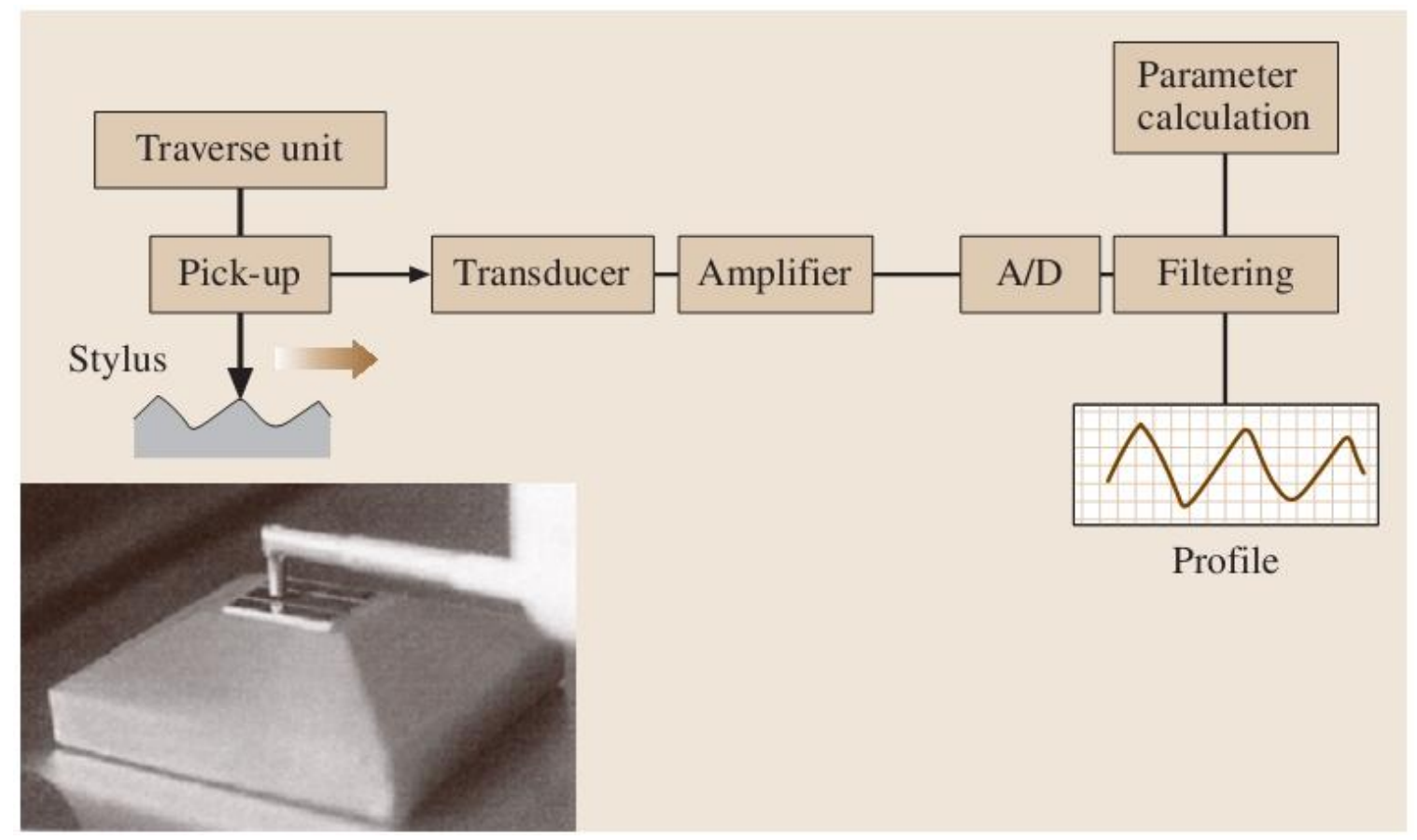

Figure 23 - One type of stylus profilometer configuration uses a pick-up to drag the stylus across the surface, and then height changes are measured using a transducer that can convert the analog signals into electrical signals. ${ }^{26}$

The radius of the diamond tips can range from 1 to $10 \mu \mathrm{m}$ and can achieve resolutions on the nanometer scale. The contact force that is applied to the surface can be varied between 0.1 and $10 \mathrm{mg}$, depending on the type of material that is being characterized. For softer films, a smaller force is generally used so as to not scratch the film's surface. The importance of nondestructive testing came around in the 1960s and 1970s with the microelectronics industry that often had soft, high quality films and surfaces that are unacceptable if they are scratched. This need for nondestructive testing also resulted in the invention of optical profilometers that did not even have to touch the film's surface. For harder films, a larger force is used because it can withstand more pressure than the softer films before becoming scratched. An advantage of contact profilometry is that it is not as susceptible to contaminates and debris when it measures the 
surface. In non-contact and optical profilometer techniques they can often measure the contaminants instead of the surface itself, throwing off results and calculations.

To understand the calculations that a profilometer performs, it is useful to understand the microscopic nature of a surface. An ideal surface would be completely flat with no height changes or roughness, but unfortunately there are no ideal surfaces in the real world. A surface is made up of macrodeviations, waviness, roughness, and microroughness (Figure 24).

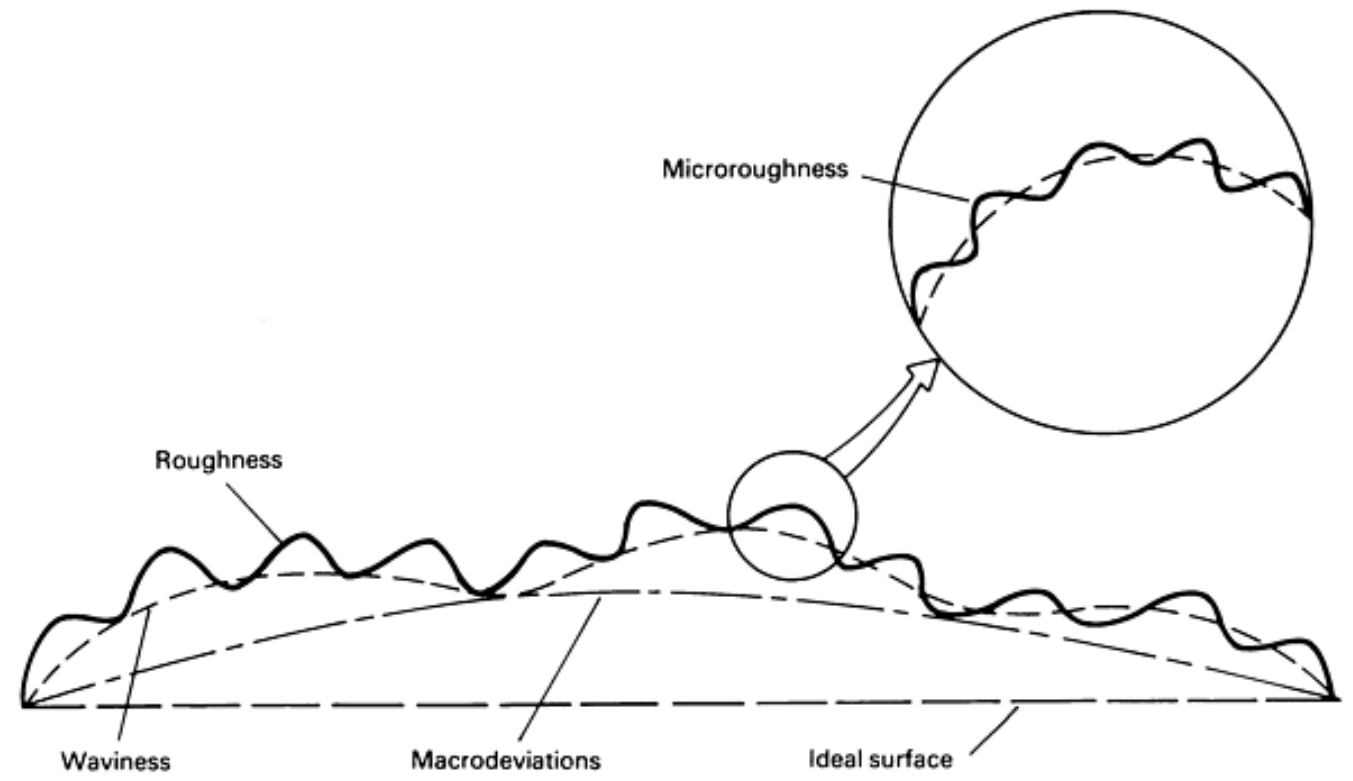

Figure 24 - In the microscopic scale, a typical surface contains macrodeviations, waviness, roughness, and finally microroughness. ${ }^{27}$

Although some of these definitions pertain to surfaces that are machined with equipment like CNC mills, they are still useful for understanding the ideas. Macrodeviations are large errors in the surface that are undesired and are departures from the designed profile that are often caused by a lack of accuracy or stiffness in the machine system. Waviness is periodic deviations in the surface that could be caused by low-level oscillations of the machining system. The 
waviness wavelengths can range from 1 to $10 \mathrm{~mm}$ and the height can range from a few to several hundred micrometers. ${ }^{27}$ Roughness is the deviations on the wavy surface itself and can be caused by the wearing of a cutting tool, machining conditions, or the microstructure of the workpiece. Microroughness is the deviations superimposed on the surface roughness and may extend down to the atomic scale. A profilometer will typically measure and calculate roughness and waviness, which are represented by the variables $R$ and $W$. The average roughness parameter, $R_{a}$, is most commonly used worldwide to measure surface texture and is typically expressed in units of height. The root mean square roughness, $R_{q}$ and sometimes $R_{R M S}$, is also used, but more commonly for modeling purposes.

\subsubsection{Optical Properties}

The optical properties of the $\mathrm{ZnO}$ thin films, such as the film thickness and band gap, were determined using a technique called spectral reflectance or thinfilm interference. The advantages of using an optical technique are that the measurements are quick, accurate, non-destructive, and require little to no sample preparation. Spectral reflectance is an optical technique that measures how the films interact with the light from the light source and can be used to measure thickness, roughness, and optical constants like the index of refraction and extinction coefficient. ${ }^{28}$ Reflectance, which is often used interchangeable with reflectivity, is the amount of incident light that is reflected by a surface. The index of refraction of a material is a number that indicates the speed at which light moves through a material compared to how it moves through a vacuum. The 
extinction coefficient is an imaginary portion of the index of refraction that indicates the absorption loss when the wave passes through the film. These optical constants give information about how the light moves through the film and reflects off the material and can be used to measure the band gap of the material.

Spectral reflectance starts with a light source pointed nearly perpendicular to the film's surface. The light emitted from the light source penetrates through the air and into the film's surface, which causes the light to reflect, absorb, and transmit through the film (Figure 25). Where $n_{1}$ is the index of refraction of the air, $n_{2}$ is the index of refraction of the $\mathrm{ZnO}$ film; $\theta_{1}$ is the angle of the incident light, $\theta_{2}$ is the angle of the transmitted light, and $d$ is the thickness of the film. These variables are used when calculating the optical constants of the film. When measuring the thickness of the film with a Filmetrics F20, the incident light is perpendicular to the surface and therefore the angle of the incident light is $0^{\circ}$. If the angle of the incident light is non-normal, then the technique is called ellipsometry. Ellipsometry is a more advanced optical technique that allows the reflectance measurement of more complex structures and at two different polarizations, but requires more expensive equipment. ${ }^{28}$ Once the light hits the $\mathrm{ZnO}$, it will simultaneously reflect and transmit through the thin film and then again transmit and reflect at the next interface if possible. The light that is reflected through the upper and lower boundaries of the film will interfere with one another and form a new wave that is sent back to the spectrometer with information about the film's thickness and the refractive index. 


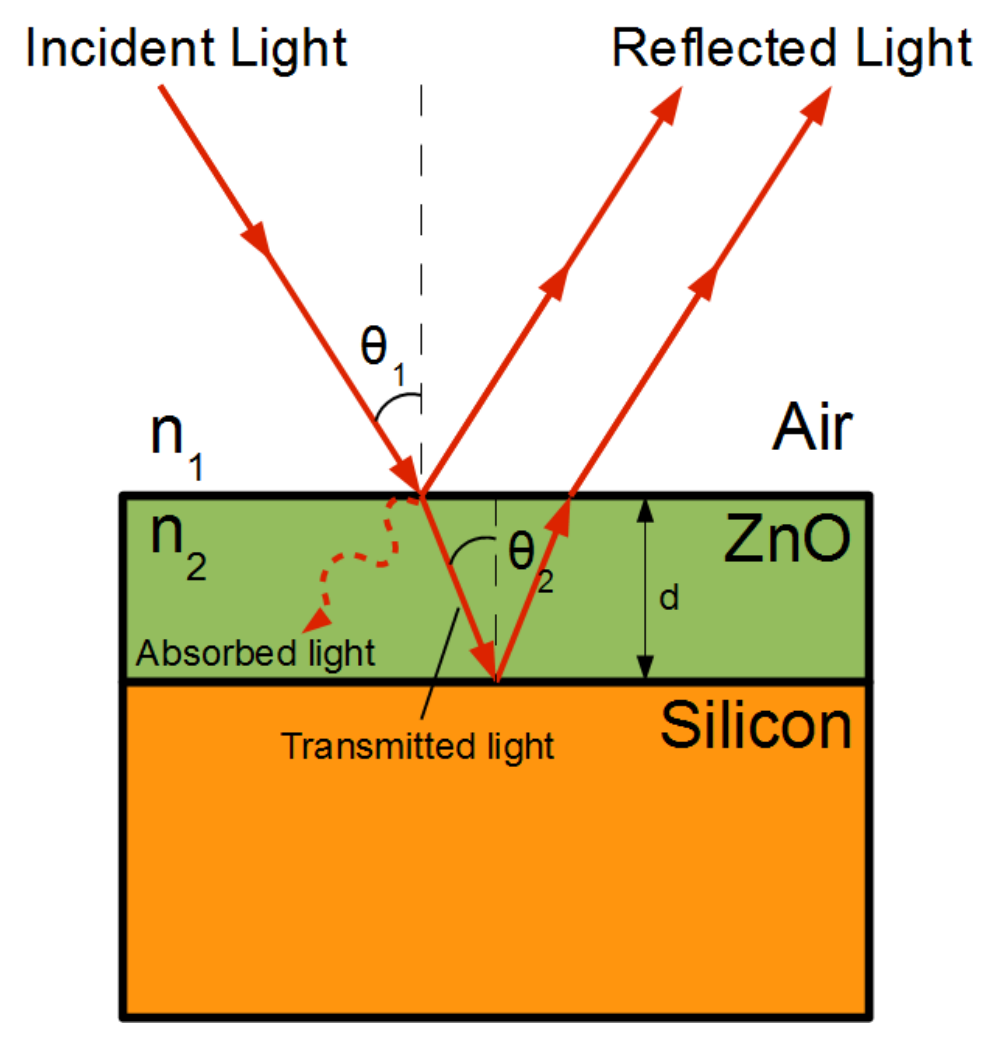

Figure 25 - To measure the ZnO film's reflectance, an incident light is direct to the surface of the film, where it transmits into the film and reflects at both the upper and lower boundaries. The resulting reflected waves cause either destructive or constructive interference and are sent back to the spectrometer for analysis.

The film's thickness, optical constants, and roughness will determine the amplitude and periodicity of the measured reflectance spectrum (Figure 26). The reflectance spectrum is a film's percent reflectance versus the wavelength of light, where 1 is equivalent to $100 \%$ reflectance. A film's properties are determined by using mathematical models that tell about how a material's index of refraction $(n)$ and extinction coefficient $(k)$ change over a range of wavelengths. The mathematical model uses theoretical values for thickness, $n$, and $k$ that are used to calculate a theoretical reflectance spectrum and adjusts the calculated spectrum until it matches the measured reflectance spectrum. ${ }^{28}$ How well the calculated and measured spectrums match each other is quantified by the goodness of fit, GOF, which is a value from 0 to 1 , with 1 being the best fit 
possible. The GOF of the measurement can often be reduced by slight amounts of surface or interface roughness, but can be accommodated by entering in estimated values for the surface roughness. Haziness in the film, which causes the spot of light to be seen, generally means that there is surface roughness that is greater than $200 \AA .{ }^{29}$ The mathematical model that is utilized is determined by the type of material, such as dielectric, semiconductors, metals, and amorphous materials. The Cauchy model, often called a Lorentzian distribution, was utilized for $\mathrm{ZnO}$ because its calculated spectrums fitted the measured spectrums best.

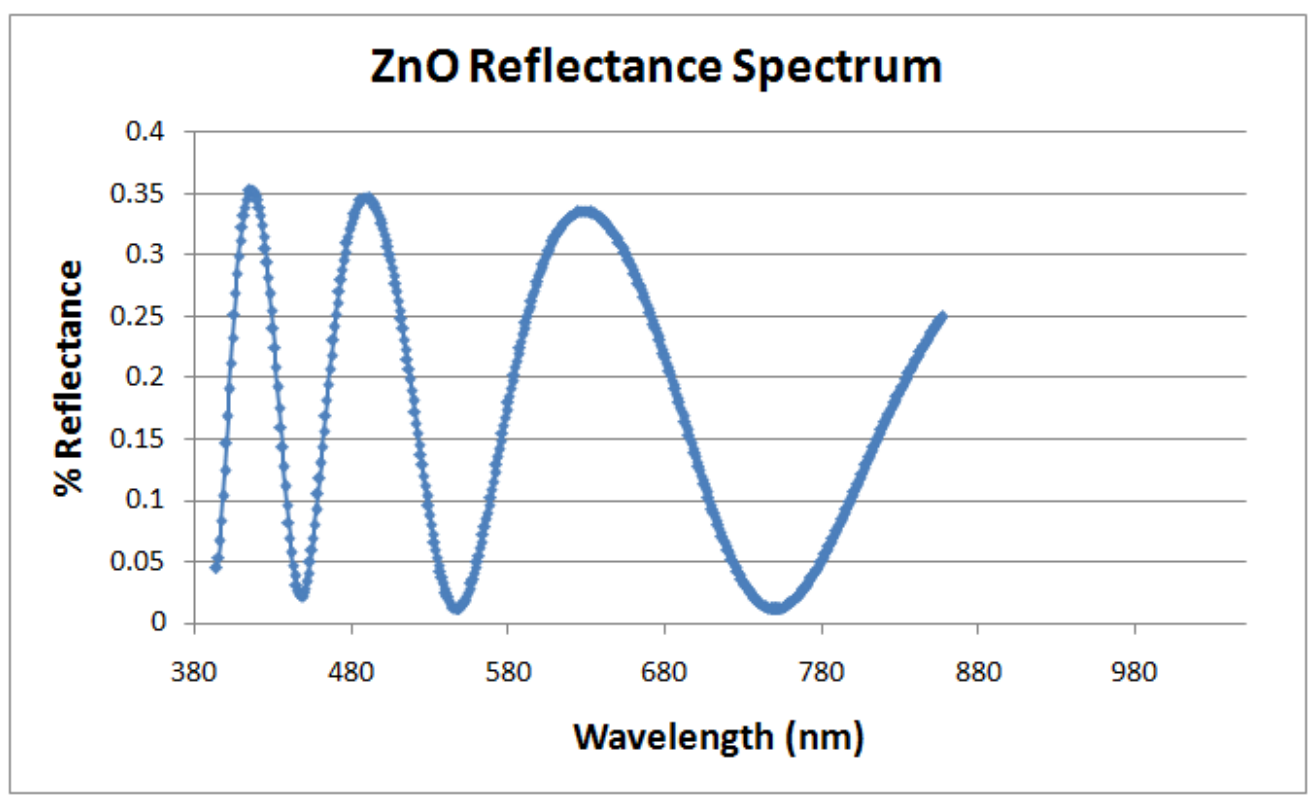

Figure 26 - The amplitude and period of the measured reflectance curve are dependent on the film's thickness, optical constants, and roughness.

The reflectance spectrum tends to form waves because of the wavelike nature of the light that is received from the reflected surface. The reflected light will interfere with one another constructively or destructively depending on the phase relationship. The phase relationship of the light will depend on the light's path length back to the spectrometer and the path length is determined by the 
thickness of the film. The thicker the films, the more oscillations there will be in the reflectance spectrum (Figure 27). ${ }^{28}$

\section{Determination of thickness (d)}
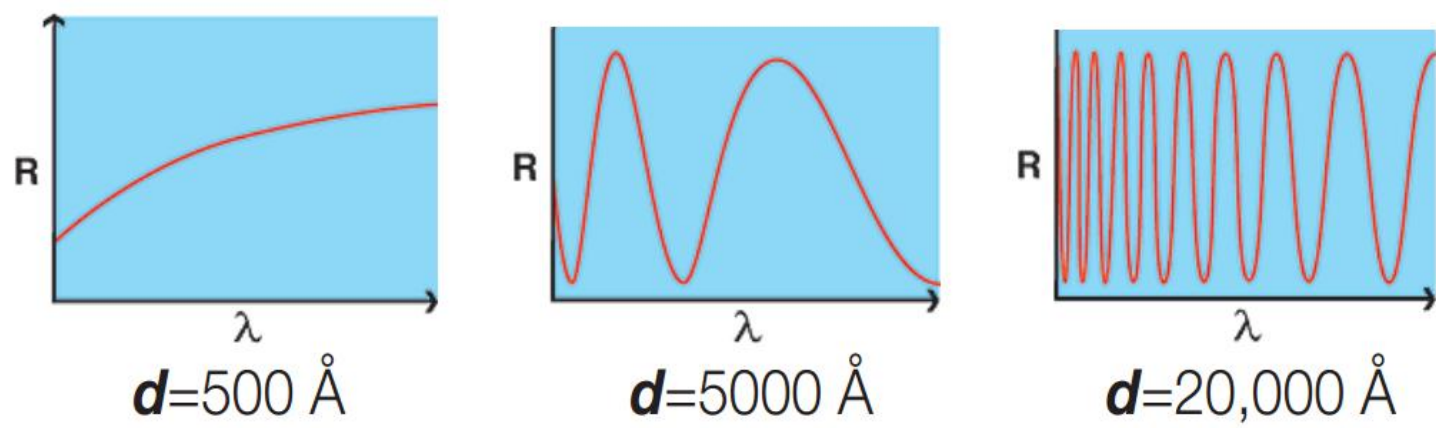

Figure 27 - As the thickness of the film increases, so do the number of oscillations in the reflectance spectrum because of the wavelike nature of the light and the increased path length that the reflected light must take.

Large changes of the index of refraction are an indication of some differences in the bonding and thus the polarizability of the material. One can obtain good reproducibility of the value of the index of refraction in bulk amorphous materials, but in thin films the refractive index depends considerable on their growth mechanism and thermal history. ${ }^{30}$

\subsubsection{Optical Band Gap}

$\mathrm{ZnO}$ typically has a wide optical band gap of around $3.3 \mathrm{eV}$ at room temperature, which provides it with high electrical breakdown voltages, low electronic noise, the potential for high power input, and high operating temperatures. ${ }^{10}$ Reflectance data and the optical constants, which are easily obtained for thick films, can be used to calculate the band gap of the material. To understand how the optical band gap can be calculated through reflectance measurements and optical constants, it is useful to understand the following 
relationship between the absorption coefficient, $\alpha$, and the band gap, $E_{g}$ (Equation 3). ${ }^{31}$

$$
\alpha h v=A\left(h v-E_{g}\right)^{1 / 2}
$$

Equation 3 - This relationship between a direct band gap material and its absorption coefficient can be used to determine the band gap of the material. ${ }^{31}$

Where $\alpha$ is the absorption or attenuation coefficient, $h$ is Planck's constant $\left(4.136 \times 10^{-15} \mathrm{eV} \cdot \mathrm{s}\right), v$ is the frequency of a photon, $A$ is a constant that is different for different transitions, and $E_{g}$ is the band gap of the material. From this equation and the Tauc relation for direct band gap materials ${ }^{32}$, comes a graph of the photon energy $h v$ (abscissa) and $(\alpha h v)^{2}$ (ordinate), which results in what is called the Tauc plot (Figure 28). ${ }^{31}$ 


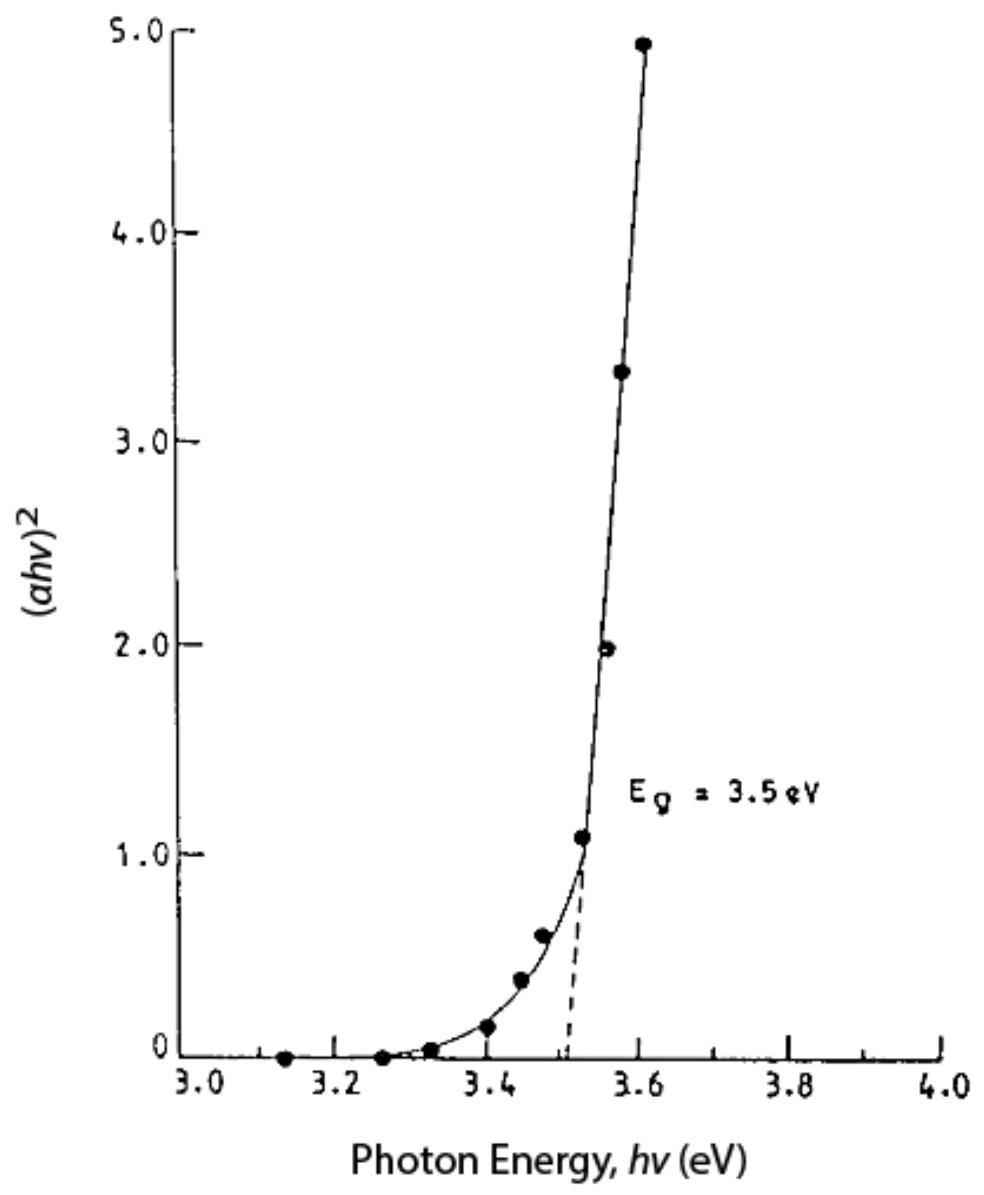

Figure 28 - A typical Tauc plot of ZnS, showing how the optical band gap of a material can be determined by extrapolating the linear portion of the curve to the $\mathrm{x}$-axis. ${ }^{31}$

From the Tauc plot, the optical band gap can be determined by extrapolating a straight line from the linear portion of the curve until it intersects with the $x$-axis. The energy value that the line intersects with on the $x$-axis is the optical band gap of the material. ${ }^{31}$ However, in order to create the Tauc plot the absorption coefficient, $\alpha$, needs to be determined. The absorption coefficient can be determined by using the extinction coefficient, $k$, which is the imaginary part of the refractive index and the following relation (Equation 4). ${ }^{33,34}$ 


$$
\begin{gathered}
k=\frac{\alpha \lambda}{4 \pi} \\
\alpha=\frac{4 k \pi}{\lambda}
\end{gathered}
$$

Equation 4 - Once the relation is rearranged, the absorption coefficient can be determined and thus the Tauc plot for $\mathrm{ZnO}$ can be created for each sample. ${ }^{33,34}$

Where $\alpha$ is the absorption coefficient, $k$ is the extinction coefficient, and $\lambda$ is the wavelength of light in meters. The reflectance and optical constants are determined using a Filmetrics F20 thin-film analyzer and the measurements result in reflectance values, refractive indices, and extinction coefficients at wavelengths between $380 \mathrm{~nm}$ and $900 \mathrm{~nm}$. The photon energy, $h v$, is determined using the Planck relation or Planck-Einstein equation between the energy of a photon $(E)$ and the frequency $(v)$ of its associated electromagnetic wave (Equation 5).

$$
E=h v=\frac{h c}{\lambda}
$$

Equation 5 - The Planck relation or Planck-Einstein equation that relates a photon's energy $(E)$ with the frequency $(v)$ of its electromagnetic waves, or inversely with its wavelength $(\lambda)$ and the speed of light $(c)$.

Once the absorption coefficient and the photon energy are known, the Tauc plot for the $\mathrm{ZnO}$ samples can be created and in the same way as before, the optical band gap of $\mathrm{ZnO}$ can be determined. It has been found that the optical energy band gap is dependent upon the thickness of film, meaning that thinner samples will have higher optical band gaps and thicker samples will have lower band gaps. It is believed that this occurs as a result of differences in grain size, lattice strain, and defect states. ${ }^{35}$ 


\subsubsection{Electrical Properties}

The electrical properties of thin films, such as electrical conductivity and resistivity, are most commonly performed in the semiconductor industry using a four-point probe system. The four-point probe is often used on test structures throughout the semiconductor fabrication process to ensure and verify the condition of the device between the various processing steps. The resistivity measurement can also be used to quantify the active doping concentration and mobility of the semiconductor. A four-point probe uses four electrically conductive electrodes that are set up to separately carry current and sense voltage. The most common layout is to have the four pins lined up linearly with the outer two electrodes injecting current into the film and the two inner electrodes measuring the resulting electrical potential (Figure 29) ${ }^{36}$ The benefit of injecting current and measuring voltage separately is that the contact resistance between the metal electrodes and the underlying material will not appear in the resistivity

measurements. ${ }^{36}$ Since the contact resistance is often large and usually depends on the electrodes' condition and material, it is easier to interpret the measured data. Two-point probe systems must take into account contact resistance and therefore it makes the measurements much more difficult. 


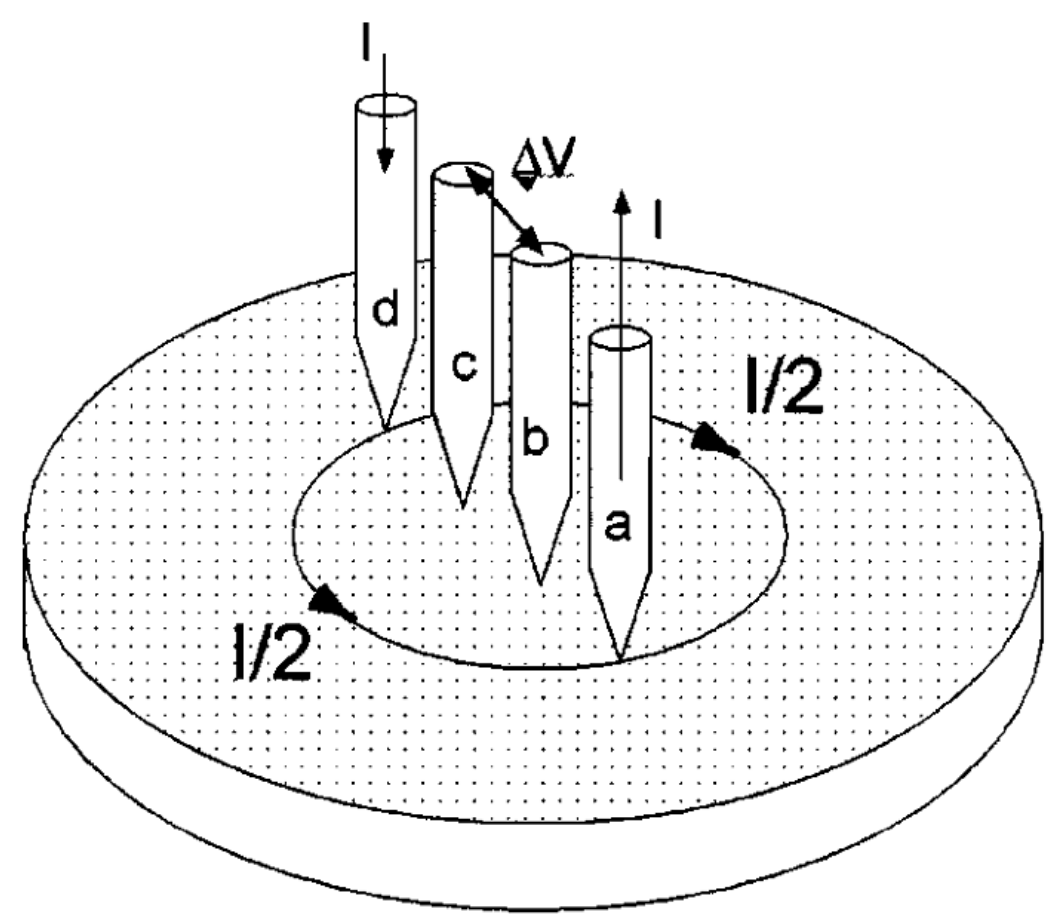

Figure 29 - Four-point probes are generally laid out in a linear fashion with the two outer electrodes carrying the current from the power supply and the inner electrodes sensing the changes in voltage. ${ }^{36}$

The reason that this setup works for calculating resistivity is because of Kelvin sensing, which uses what is called a Kelvin bridge that was developed by Lord Kelvin in 1861 in order to measure extremely small resistances below $1 \Omega$. In this measurement method, instead of using only an ohmmeter to calculate the resistance of a load, an ammeter and a voltmeter would be used together. Take for example a situation where the resistance of a piece of aluminum foil needed to be found. If you tried to measure the resistance of the aluminum using a typical ohmmeter, which is a two-wire method, you would get an incorrect resistance measurement (Figure 30). ${ }^{37}$ You would get an incorrect measurement because an ohmmeter measures all of the resistance in the circuit, which includes the resistance of the wires as well as the resistive load. 


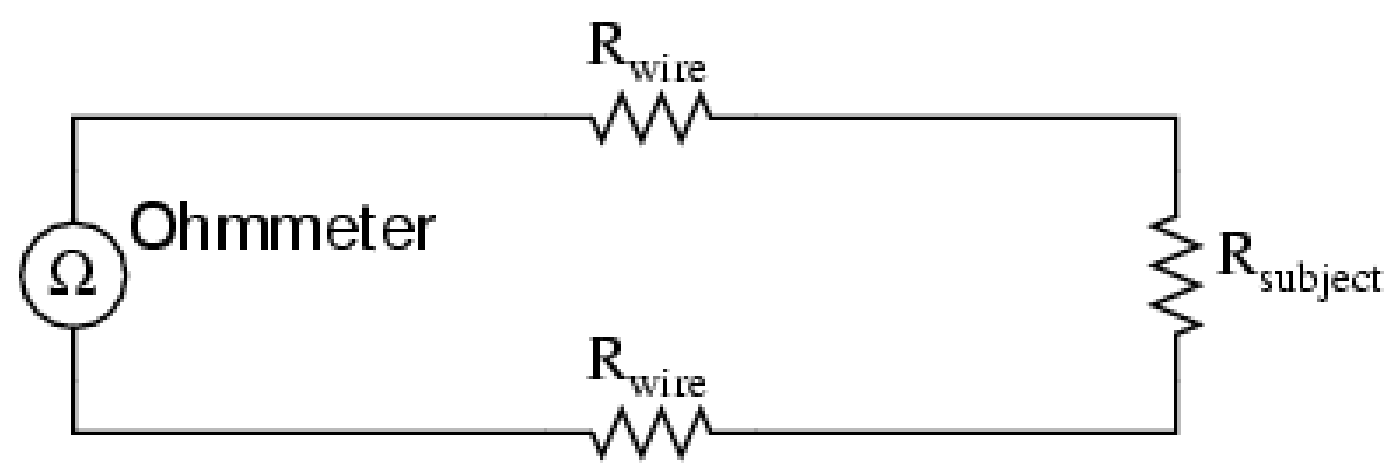

\section{Ohmmeter indicates $R_{\text {wire }}+R_{\text {subject }}+R_{\text {wire }}$}

Figure 30 - In a two-wire method an ohmmeter would be used to directly measure the resistance of the component, $R_{\text {subject, }}$ but would include the resistance of all of the components in the circuit. ${ }^{37}$

Instead, you would connect a DC power supply to both ends of the piece of aluminum. Then you would dial in the current you want to use and apply it to both ends of the aluminum. Then you would put a voltmeter in the circuit and measure the voltage drop across the aluminum (Figure 31$).{ }^{37}$

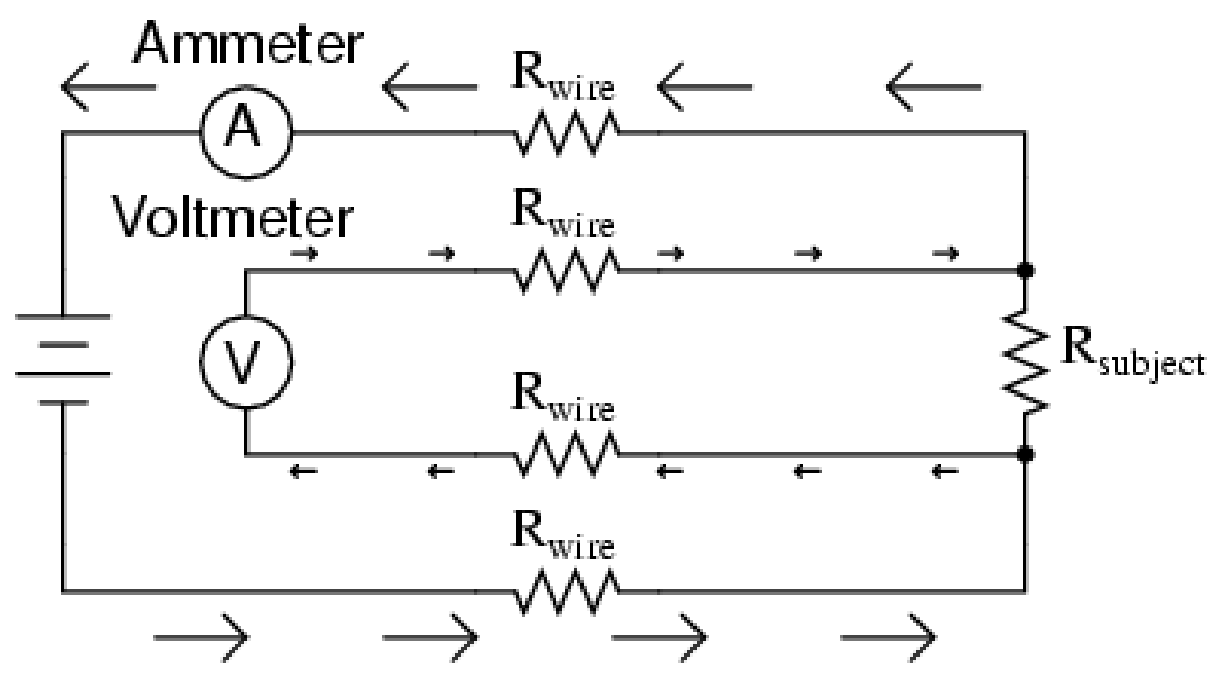

Figure 31 - The four-wire measurement method uses both an ammeter and a voltmeter to measure the resistance of a subject, thereby avoiding measurement of the other loads in the circuit. ${ }^{37}$

Using Ohm's law, where $\mathrm{R}=\mathrm{V} / \mathrm{I}$, you can calculate the wire's resistance by dividing the measured voltage by the applied current. Because this is a series loop, the current is the same throughout the entire circuit and because only the 
voltage drop across the aluminum is being measured, we receive only the resistance of the aluminum. The four-point probe uses this same technique, but instead uses four electrodes that are brought into contact with the sample's surface. The sheet resistivity of the thin film is usually calculated using Equation 6.

$$
\begin{gathered}
\rho=R C F\left(\frac{V_{\text {measured }}}{I_{\text {applied }}}\right) \\
\rho=\frac{\pi}{\ln 2} \cdot t\left(\frac{V_{\text {measured }}}{I_{\text {applied }}}\right)
\end{gathered}
$$

Equation 6 - To measure the sheet resistivity of the thin film, the resistivity correction factor (RCF) is multiplied by the measured voltage over the applied current.

Where $\rho$ is the sheet resistivity of the thin film, RCF is the resistivity correction factor, $t$ is the thickness of the measured film, $V_{\text {measured }}$ is the measured electrical potential between the two inner electrodes, and the $I_{\text {applied }}$ is the current that was applied to the thin film from the outer electrodes. The resistivity correction factor takes into account the size of the test structure, the thickness of the material being measured, the size of the electrodes, and the position of the electrodes with respect to the boundaries of the test structure ${ }^{36}$ When using the four-point probe on an infinite thin sheet, the RCF is $\frac{\pi}{\ln 2} t$.

\subsubsection{Piezoelectric Properties}

There are a variety of methods for characterizing the piezoelectric properties of piezoelectric thin films, but all of the methods require extremely high sensitivity and accuracy to detect the small displacements of the piezoelectric thin films. Initially, the fabricated piezoelectric $\mathrm{ZnO}$ microspeaker would have been used to 
characterize the ZnO's piezoelectricity because there is a simple relationship between the speakers sound pressure level (SPL), the displacement that the microspeaker diaphragm experiences, and the applied voltage. Unfortunately, the microspeaker fabrication was unsuccessful and therefore an alternative technique was required. One of the most common techniques is called interferometry, which can measure the surface displacement of thin films by applying an $\mathrm{AC}$ voltage to the piezoelectric film and then firing a He-Ne laser at the surface. The reflected laser beam is modulated by the surface displacement of the piezoelectric film and is sent to a photodetector, a signal processor, and a spectrum analyzer that can interpret the data. Through a simple equation, this method will give a quantitative measurement of the thin film's piezoelectricity. Unfortunately, access to an interferometer is unavailable. Therefore another method called piezoresponse force microscopy (PFM) was utilized to measure and quantify the piezoelectricity of the deposited $\mathrm{ZnO}$ thin films.

\subsubsection{Piezoresponse Force Microscopy (PFM)}

An AFM technique called piezoresponse force microscopy (PFM) was utilized to characterize ZnO's piezoelectric properties. An Asylum Research MFP-3D AFM along with AC240TM ElectriLever conductive cantilevers were used to obtain the data. In the typical AFM contact imaging mode, a microfabricated silicon or silicon nitride cantilever with a sharp tip touches the surface and rasterscans the surface while its deflection and oscillation amplitude is measured. The measurements are usually performed with an optical tracking system that reflects a laser off of the back of the cantilever into a position sensitive detector (Figure 
32). ${ }^{38}$ When changes in the deflection or oscillation that are different then the specified setpoint voltage are measured, a piezoelectric stack actuates and corrects the cantilever back to the setpoint. The voltages that are sent to the correction piezo are recorded and used to determine the height at a specific $\mathrm{XY}$ position.

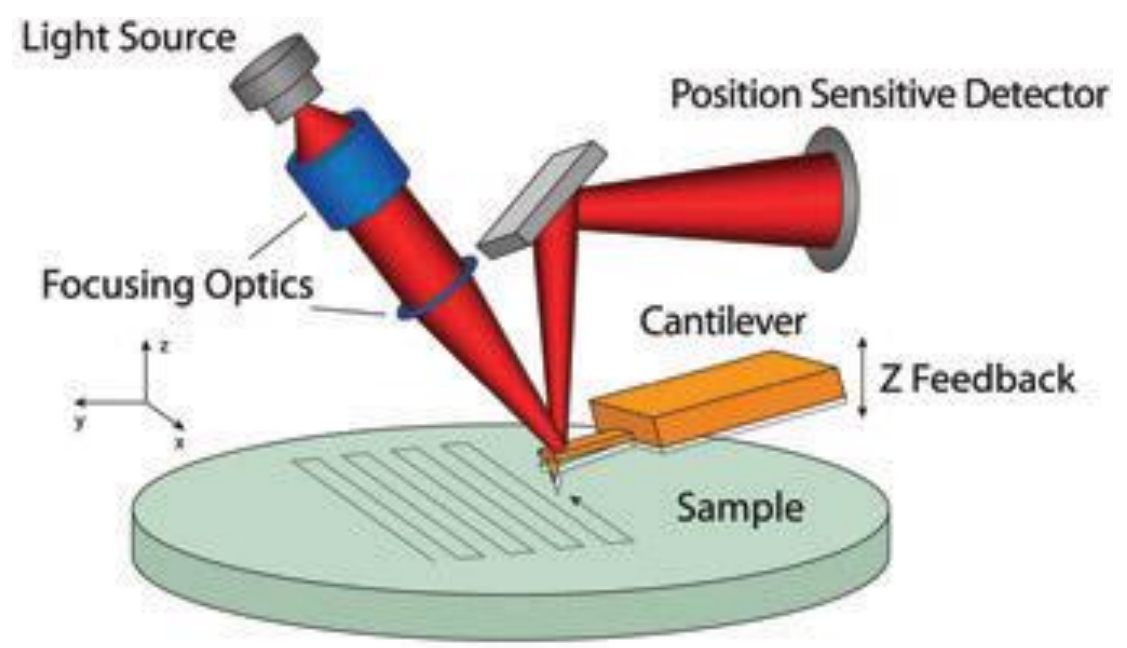

\section{Unrestricted Optical Access from Below the Sample Plane}

Figure 32 - In a typical AFM, a silicon cantilever with a sharp tip is raster-scanned across the surface of a sample while the deflection and oscillation amplitude of the cantilever is measured with an optical tracking system. $^{38}$

PFM is similar to typical contact mode imaging except an AC voltage is applied to the surface of a piezoelectric sample through the tip of a conductive AFM cantilever. In reaction to the applied voltage, the piezoelectric thin film changes shape and causes the "tip-to-surface contact" to experience a height change. Because the cantilever is in contact with the surface, the height change can be tracked and measured as the tip is scanned over the surface. This height change is usually tracked with a piece of equipment called a lock-in amplifier. The lock-in amplifier tracks the contact resonance frequency of the tip-to-surface contact. In Asylum's MFP-3D, this lock-in amplifier system is built into the AFM's 
controller. The MFP-3D system also has a special technique for measuring piezoelectricity called Dual AC Resonance Tracking (DART) that makes it easier to measure the small displacements of the thin film. DART-PFM is the mode utilized because it results in better signal-to-noise ratio than in a single AC mode. In DART-PFM, two AC signals are sent to the cantilever, which causes the piezoelectric surface to change shape and actuate the cantilever (Figure 33). ${ }^{39}$ DART-PFM uses the contact resonance of the tip and surface to enhance or amplify the received piezoresponse signal.

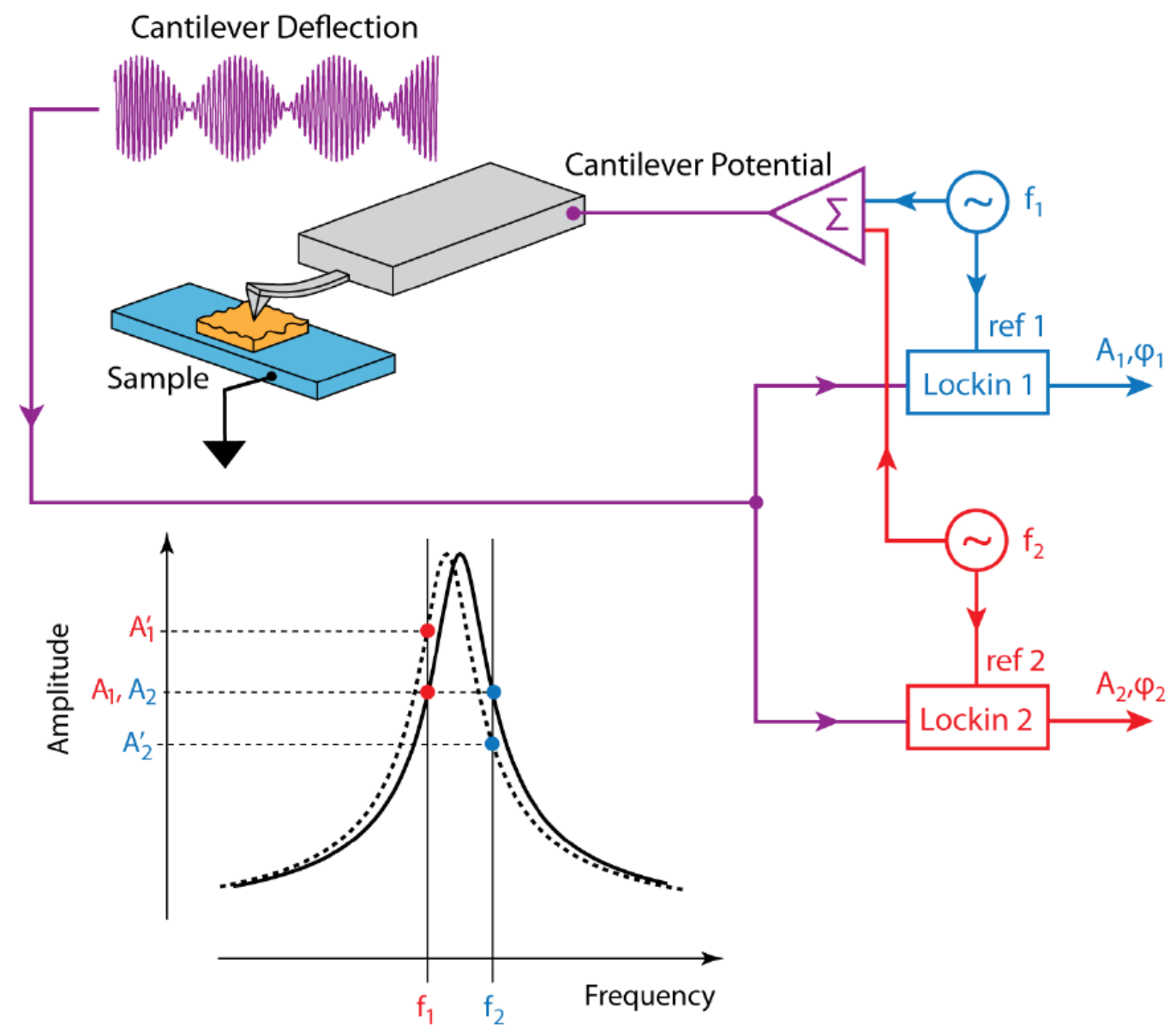

Figure 33 - DART-PFM on the MFP-3D atomic force microscope involves applying two AC voltages to the tip to sample contact, which causes the surface of the piezoelectric to change shape. The cantilever's deflection and contact resonance frequency is tracked and measured using two lock-in amplifiers. 
This tip-to-surface contact and the way that it is modeled is an important part of the piezoelectric calculations that are performed in the software. The tip-tosurface contact is modeled as a damped harmonic oscillator (DHO), which is composed of a spring and a dashpot (Figure 34$).{ }^{39}$

(a)

(b)

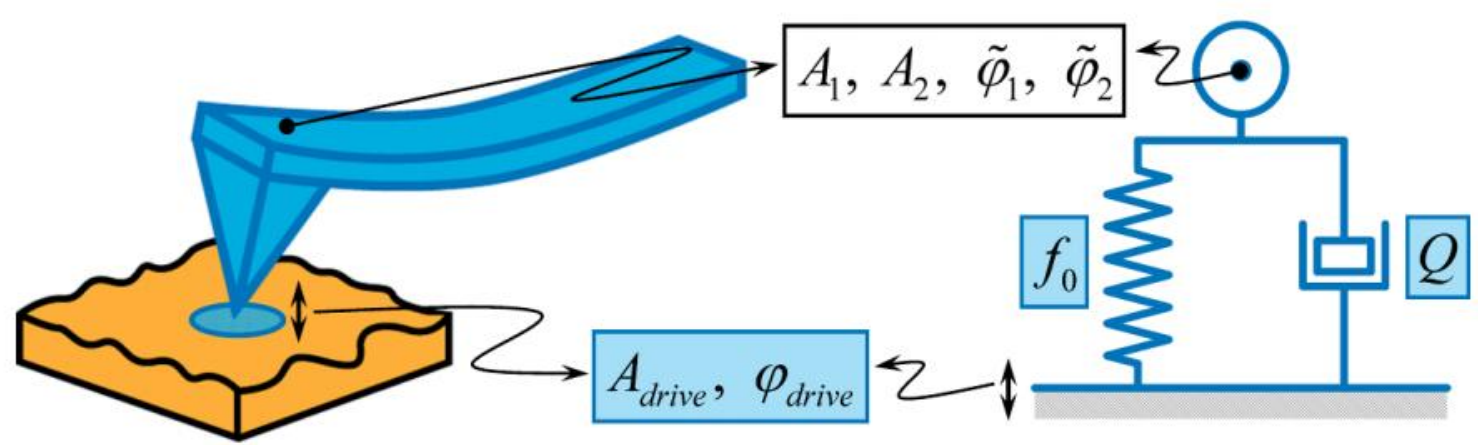

Figure 34 - The tip-to-surface contact between the piezoelectric thin film and the conductive cantilever is modeled as a damped harmonic oscillator. (a) Shows the tip-to-contact system, involving the piezoelectric film and the cantilever and the way that it oscillates when an AC voltage is applied. (b) Shows how the system is modeled as a $\mathrm{DHO}^{39}$

In this model, the dual AC excitation voltages result in sample surface motion with amplitude $\left(A_{\text {drive }}\right)$ and phase $\left(\varphi_{\text {drive }}\right)$. The resonance frequency $f_{0}$ and quality factor $Q$ represent the viscoelastic nature of the tip-sample contact dynamics. $A_{\text {drive }}, \varphi_{\text {drive }}, f_{0}$, and $Q$ are four unknown parameters and can be calculated using the four measurements $A_{1}, A_{2}, \tilde{\varphi}_{1}$, and $\tilde{\varphi}_{2}$. Depending on the imaging technique being used, the unknown parameters will carry different information about the material properties of the surface. In PFM, the $A_{\text {drive }}$ is linked to the local piezoelectric coefficient of the sample and $\varphi_{\text {drive }}$ gives information about the polarization direction of the crystals. The $d_{33}$ is determined from dividing the $A_{\text {drive }}$ by the voltage applied to the conductive tip. The process of obtaining the $d_{33}$ is explained in more detail in section 2.2.6. 


\section{Chapter 2: Experimental Procedures}

Initially, the goal of this thesis was to fabricate a microspeaker structure using a $\mathrm{ZnO}$ thin film as the actuator for the microspeaker. Multiple microfabrication processing steps were required to fabricate the microspeaker structure, which consisted mainly of a thin silicon diaphragm approximately $10 \mu \mathrm{m}$ thick. Several problems were encountered during the processing of this silicon diaphragm, which led to alternative designs to the microspeaker that will be explained later in more detail. After numerous setbacks and design changes, the scope of the project changed instead from device fabrication to the characterization of the $\mathrm{ZnO}$ thin film in order to understand more about its material properties. However, the microfabrication techniques utilized were not performed in vain since similar techniques would be used for the fabrication of a working device. This section will first cover the procedures that were utilized to fabricate the microspeaker devices and will then cover the characterization procedures.

\subsection{Device Processing and Fabrication}

Fabrication of the microspeaker started with a $100 \mathrm{~mm}$-type $\{100\}$ oriented silicon wafer (Figure 35). This type of wafer was chosen because they are test wafers that are inexpensive and in abundance and will not be missed if broken. Because the microspeaker will be actuated by a $\mathrm{ZnO}$ thin film sandwiched between two aluminum electrode layers, the microspeaker structure requires processing of both sides of the wafer. This also means that the etched diaphragms must be aligned with the $\mathrm{ZnO}$ structure on the other side of the 
wafer. A summary of all of the processing steps involved in the fabrication are detailed in a cross-sectional step-by-step diagram (Figure 36).

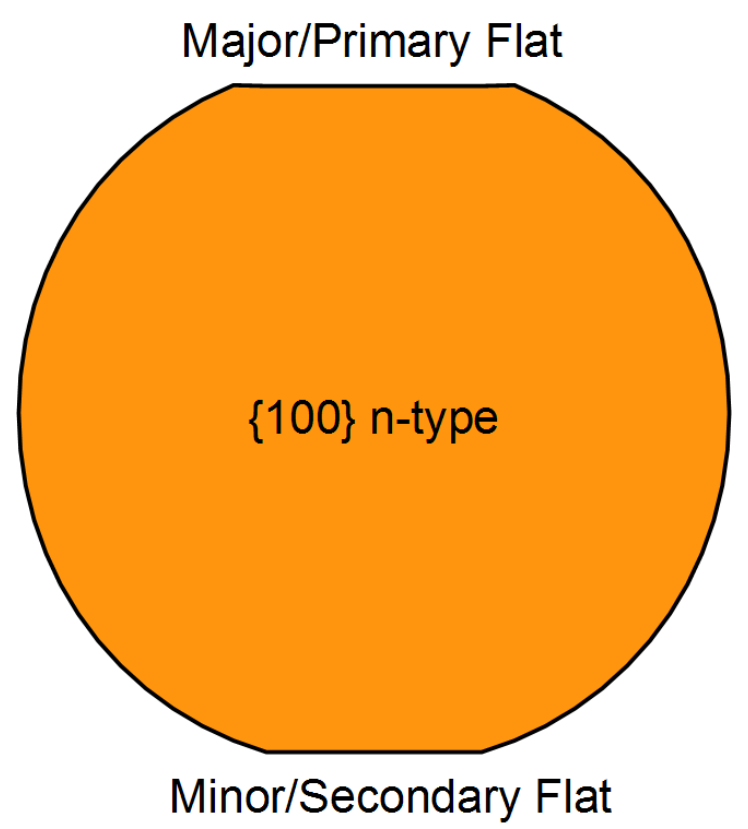

Figure 35 - The single crystal silicon test wafers that were used to fabricate the microspeaker structures were 4 inch wafers with $\{100\}$ orientation and n-type doping. 
1

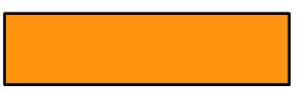

Clean bare wafer in BOE and Piranha

2

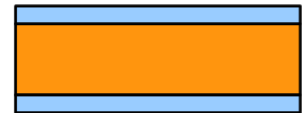

Thermal oxidation, forming $\mathrm{SiO}_{2}$

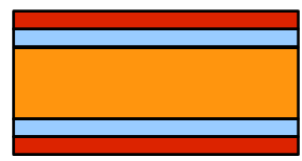

BOE Dip $\rightarrow$ Spin

Coat PR $\rightarrow$ Soft

Bake $\rightarrow$ Expose

4

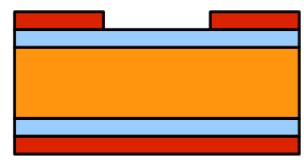

Develop PR $\rightarrow$ Hard

Bake

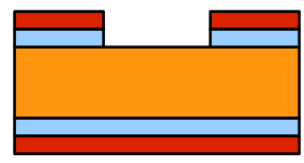

$\mathrm{SiO}_{2}$ Etching in $\mathrm{BOE}$

6

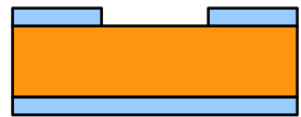

Photoresist

Stripping

7

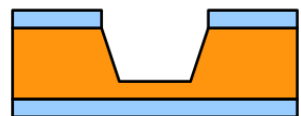

Deep Wet Etching in $25 \%$ TMAH @ $80^{\circ} \mathrm{C}$

8

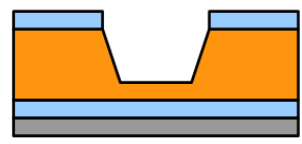

DC Sputter Aluminum Electrodes

9

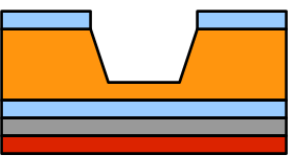

Spin Coat PR $\rightarrow$ Soft

Bake $\rightarrow$ Expose

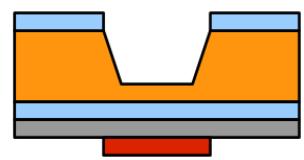

Develop PR $\rightarrow$ Hard

Bake
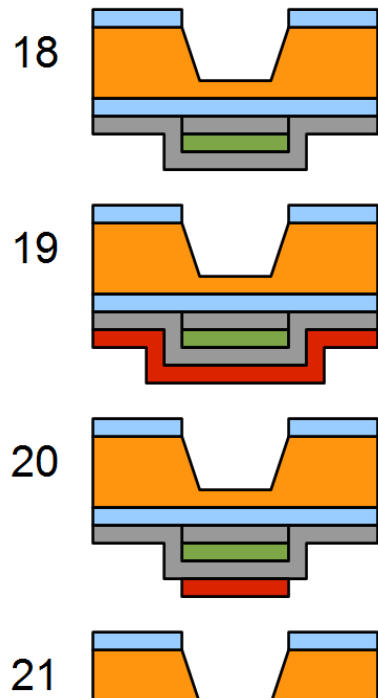

19

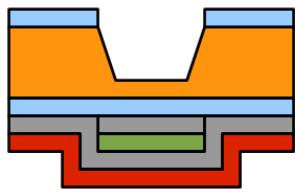

20

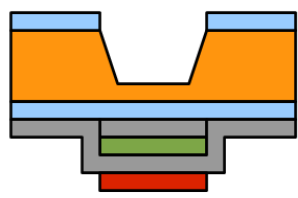

21

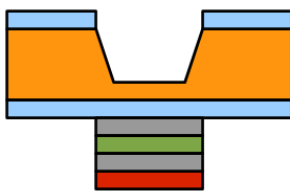

22

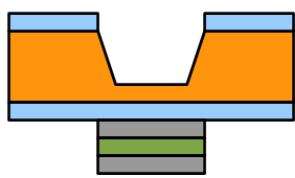

Photoresist Stripping

Etch Al electrodes in Piranha or CD-26

Developer

Photoresist

Stripping
DC Sputter $2^{\text {nd }}$ layer of Al electrodes

Spin Coat PR $\rightarrow$ Soft Bake $\rightarrow$ Expose

Develop PR $\rightarrow$ Hard

Bake

Etch Al electrodes in Piranha or CD-26

Developer

DC Sputter ZnO thin

Develop PR $\rightarrow$ Hard

Bake

Etch $\mathrm{ZnO}$ in 1 to $\mathbf{3 0}$ solution of acetic

Photoresist

Stripping
Spin Coat PR $\rightarrow$ Soft
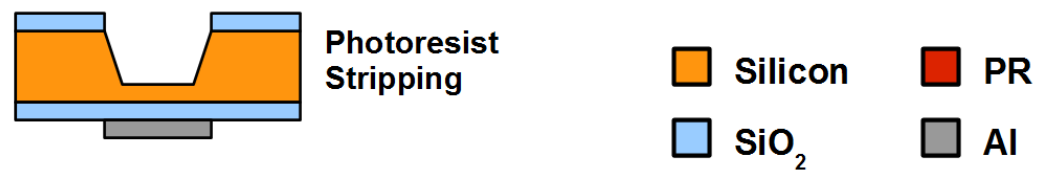

Figure 36 - To fabricate the microspeaker structure, the following processing steps had to be performed. 
The processing started with bare double-side polished silicon wafers that were cleaned of organic compounds and contamination for about 10 minutes in a piranha solution at $70^{\circ} \mathrm{C}$ (Figure 37). Piranha is a highly toxic mixture of sulfuric acid $\left(\mathrm{H}_{2} \mathrm{SO}_{4}\right)$ and hydrogen peroxide $\left(\mathrm{H}_{2} \mathrm{O}_{2}\right)$ that is a strong oxidizer and removes organic matter while also causing the wafer's surface to become hydrophobic. The piranha solution that was used has a sulfuric acid to hydrogen peroxide ratio of 9:1, but it can vary anywhere from 3:1 to $7: 1$ in other clean rooms. The hydrogen peroxide should always be added to the sulfuric acid and its concentration in the solution will determine how aggressively the solution removes organic contaminates. Organic contamination on the surface can interfere with the growth of oxide on the surface of the silicon. The organic substance could act as a thermal barrier to the silicon, resulting in less heat reaching the silicon substrate, which would cause a thinner oxide thickness in specific locations. A clean silicon surface is desired to ensure that the silicon dioxide thickness is uniform and consistent. 


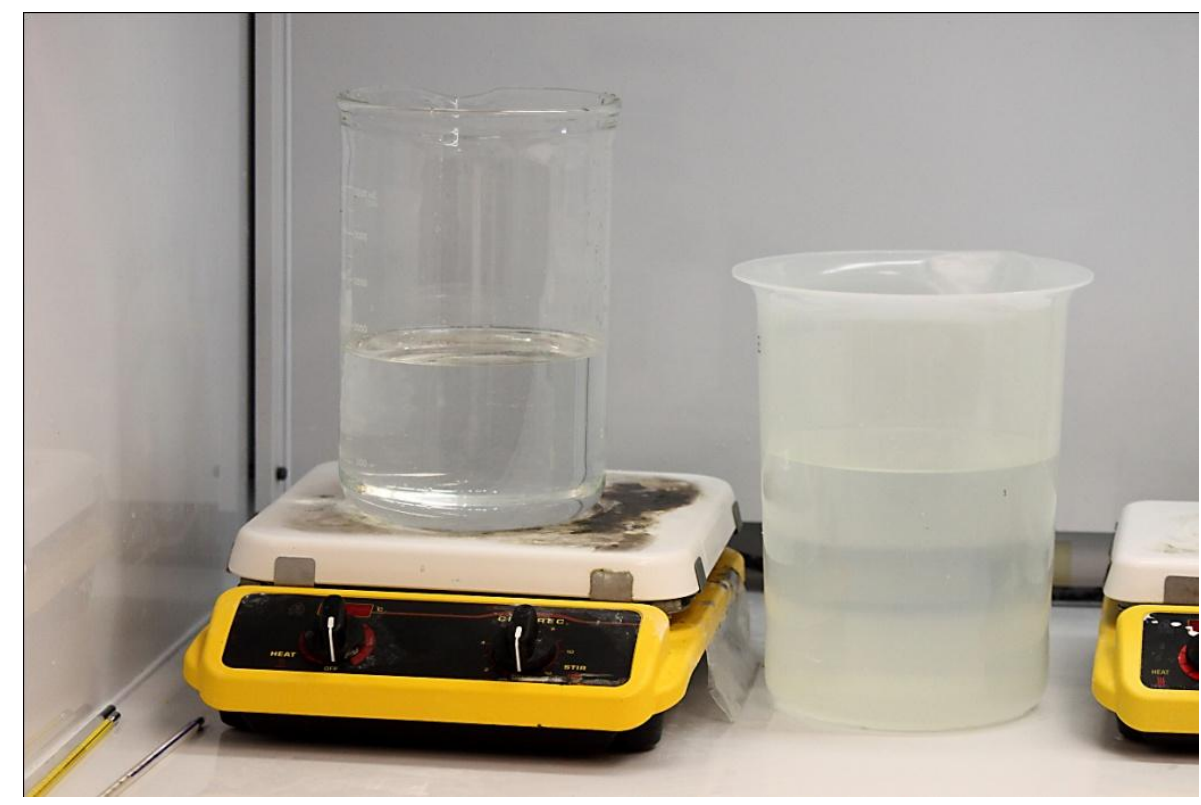

Figure 37 - Before growing a thermal oxide on the surface of the silicon wafers, the wafers were cleaned of organic contamination by dipping them in a piranha solution at $70^{\circ} \mathrm{C}$ for 10 minutes.

After being cleaned in piranha, the wafers were etched for about 10-20 seconds in room temperature buffered oxide etch (BOE) to remove any natural oxides that might have grown on the wafer when it was exposed to the atmosphere (Figure 38). BOE is a mixture of a buffering agent such as ammonium fluoride $\left(\mathrm{NH}_{4} \mathrm{~F}\right)$, water, and hydrofluoric acid (HF). Typically, the percentages in the solution are $30-40 \% \mathrm{NH}_{4} \mathrm{~F}, 1-10 \%$ hydrogen fluoride (hydrofluoric acid when in aqueous solution), and $55-65 \%$ water. ${ }^{40} \mathrm{BOE}$ is an extremely hazardous chemical in both its liquid and vapor form, causing burns and bone damage if absorbed into the skin and therefore it was handled under a vent hood with a lab coat, safety goggles, face mask, apron, closed-toe shoes, and chemical gloves. 


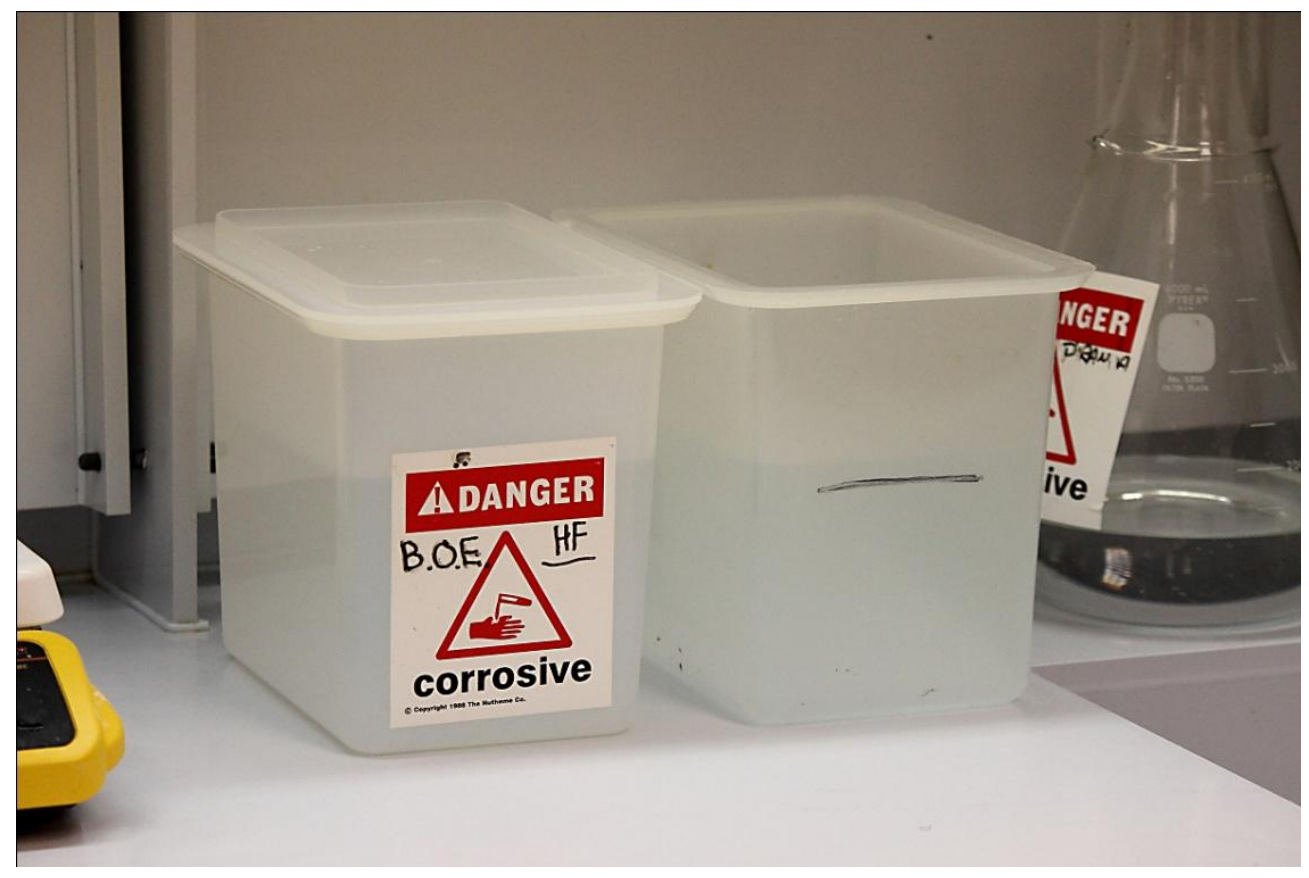

Figure 38 - After the wafers were cleaning in piranha, the native oxides on the surface of the silicon wafers were etched away by dipping them in BOE for 10-20 seconds.

After cleaning, an oxide was grown onto the surface of the double-side polished n-typed single crystal silicon wafers. This silicon dioxide layer will provide protection to the silicon from the wet etching solution, tetramethylammonium hydroxide (TMAH), which will be used to etch the diaphragm structures into the silicon. Oxide layers were thermally grown in a Thermo Products (Mini-Brute) thermal oxidation furnace on five device wafers in a furnace at $1050^{\circ} \mathrm{C}$ for 1 hour and 20 minutes (Figure 39). The device wafers in the quartz tube were surrounded by "dummy" or "junk" wafers that protected the inner wafers from heat fluctuations, allowing the oxide growth to be more evenly distributed. 


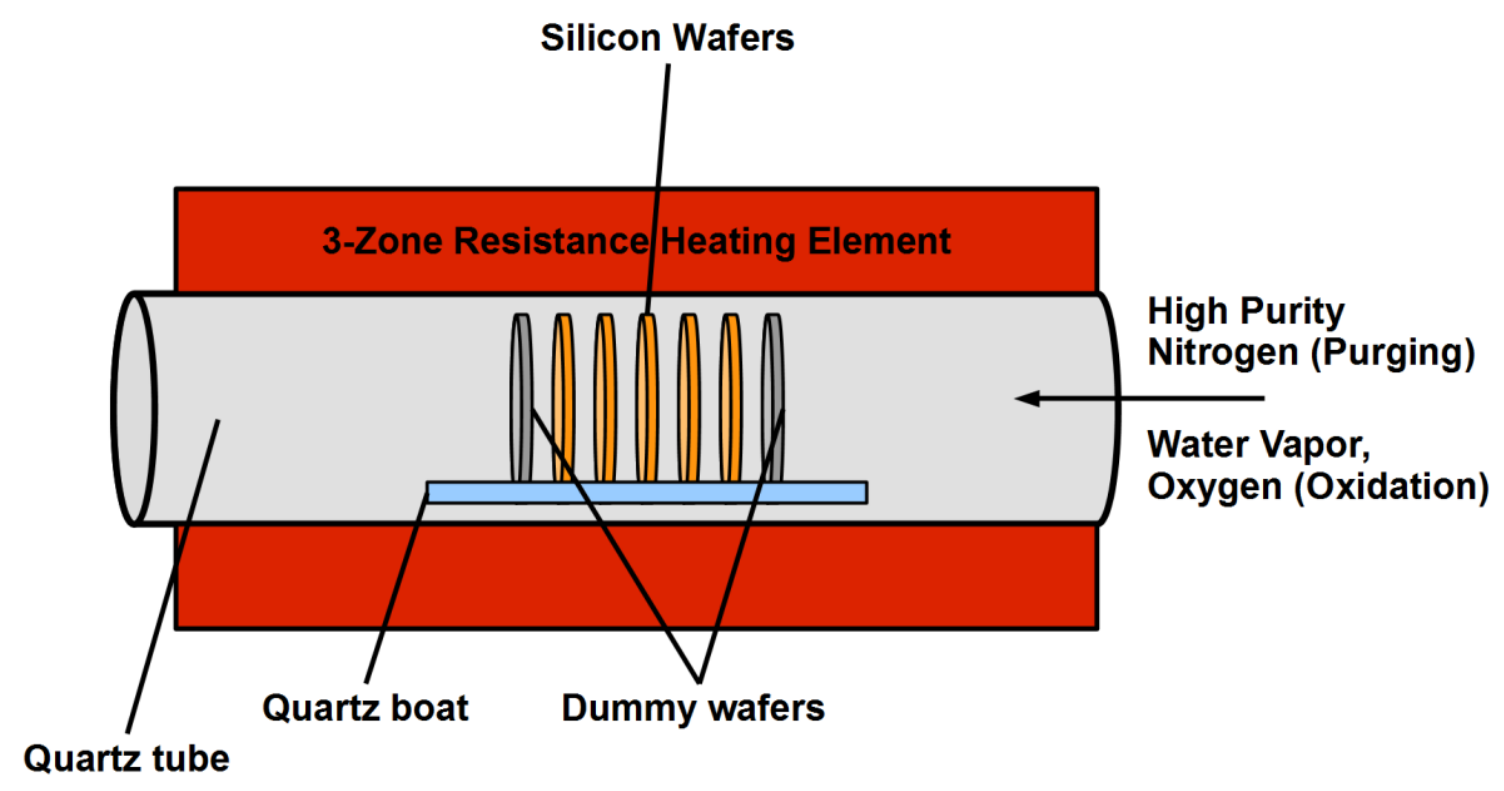

Figure $39-\mathrm{A} \mathrm{SiO}_{2}$ layer was grown on the silicon wafers using a thermal oxidation furnace that was injected with water vapor, allowing the oxidation of the silicon's surface.

Upon reaching about $200^{\circ} \mathrm{C}$ below the target temperature of $1050^{\circ} \mathrm{C}$, the wafers are slowly placed and pushed into the quartz tube on a quartz boat with a hooked quartz rod at approximately 0.25 inches/second. To avoid oxide growth during heating of the furnace, high purity nitrogen was pumped into the tube furnace at 5 LPM until reaching the final temperature. The wafers are pushed slowly to avoid thermal shock, which can cause the wafers to bow and "potato chip." Once the wafers were inside, the temperature of the furnace was increased to the final temperature of $1050^{\circ} \mathrm{C}$. Meanwhile, deionized water was heated in a large round-bottom flask with a heating mantle until boiling and bubbling. Once the furnace reached $1050^{\circ} \mathrm{C}$ and the DI water was boiling, the high-purity $\mathrm{N}_{2}$ was switched off and high-purity $\mathrm{O}_{2}$ was pumped through the bubbling DI water at 5 LPM, causing the $\mathrm{O}_{2}$ to become saturated with the water vapor. The process of "wet" oxidation or saturating the $\mathrm{O}_{2}$ with water vapor 
increases the oxide growth rate by causing the following oxidation reaction to occur.

$$
\mathrm{Si}+2 \mathrm{H}_{2} \mathrm{O} \rightarrow \mathrm{SiO}_{2}+2 \mathrm{H}_{2}(\mathrm{~g})
$$

Wet oxidation is usually the preferred and most often used method of thermal oxidation because of its increased growth rate, but this higher growth rate also leaves a "dirty" lower-density oxide that can often have dangling bonds at the silicon interface that can cause current leakage. In applications where this dielectric layer is crucial (transistors), a dry-wet-dry oxidation cycle is usually performed to get the advantages of both the increased growth rate and the purity of the dry oxidation. However, in this microspeaker device the oxide is only used as a mask and a thin dielectric layer and therefore the quality of oxide is not crucial. The time that was required to grow a $500 \mathrm{~nm}$ thick oxide was calculated using the Deal-Grove model (Equation 7).

$$
t+\tau=\left(\frac{x_{\text {oxide }}^{2}}{B}\right)+\left(\frac{x_{\text {oxide }}}{\frac{B}{A}}\right)
$$

Equation 7 - To determine the time required for thermal oxidation, the thickness of the desired oxide must be known.

Where $t$ is the required oxidation time, $x_{\text {oxide }}$ is the desired oxide thickness, $\tau$ is the time correction factor to account for any oxide already present, $B$ is $0.35 \frac{\mu m^{2}}{h r}$, and $\frac{B}{A}$ is the growth rate at $1.2 \frac{\mu m}{h r}$. No native oxides were on the wafers since they were immersed in BOE before oxidation, which etched away any oxide present on the silicon, resulting in an oxidation time of 1 hour and 18 minutes. After 1 hour and 18 minutes, the heating elements were shut off and 
nitrogen was again pumped into the tube for 20 minutes until the wafers cooled to stop more oxidation from occurring.

The thicknesses of the oxide layers were measured on a Filmetrics F20 thinfilm analyzer, which contains an integrated spectrometer with a wavelength range from 380 to $1050 \mathrm{~nm}$ and a tungsten-halogen light source. The thin-film analyzer uses spectral reflectance to take its thickness measurements by reflecting light through the oxide and analyzing the reflected light as it bounces back from the wafer's surface (Figure 40). In addition to the reflectance, the optical constants, $n$ and $k$, are also simultaneously measured and recorded. Five thickness measurements were taken across each of the device wafers and then the thicknesses were averaged.

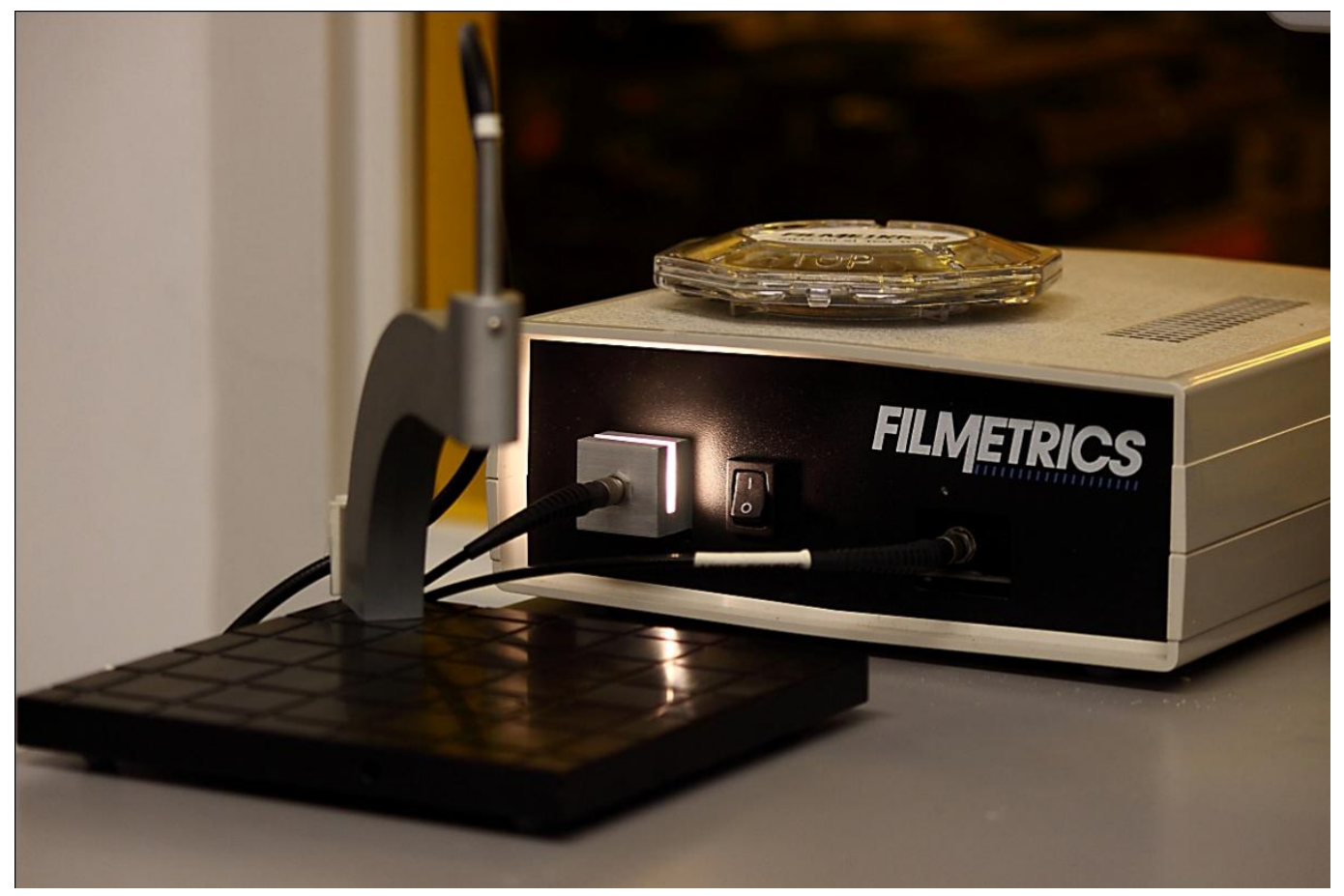

Figure 40 - A Filmetrics F20 Reflectometer, which uses spectral reflectance, was used to measure the thickness of the silicon dioxide film on the wafers. 
By growing a silicon dioxide layer on the silicon wafer, it allows the patterning of the surface with photoresist so that the silicon can be selectively etched in only certain areas (Figure 41).
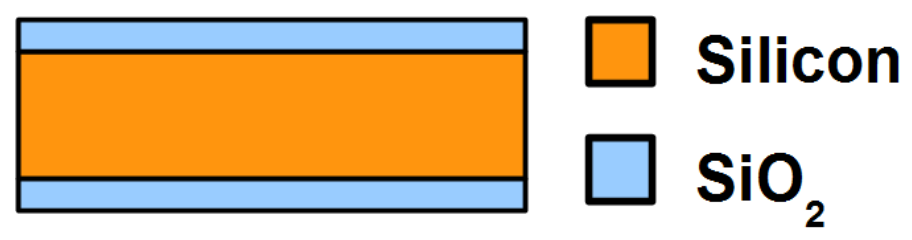

Figure 41 - The thermally grown silicon dioxide film on the silicon wafers acts as protection for the silicon during the etching process and allows the patterning of the diaphragms.

We have found that after growing an oxide, positive photoresist tends to adhere in splotches. This occurs because of surface contamination that was introduced during thermal oxidation. Therefore, the wafers were cleaned in Piranha at $70^{\circ} \mathrm{C}$ for 10 minutes and then briefly dipped in BOE for a few seconds to roughen the oxide's surface and to etch off the contaminated surface layer. Performing this BOE dip causes the photoresist to spin much more evenly. Spin coating was performed with Shipley's Microposit S1813 Positive Photoresist on a Laurell Technologies WS-400B-6NPP spin coater (Figure 42). The photoresist, which is typically stored at around $40^{\circ} \mathrm{F}$ to increase its shelf life, was left out to return to room temperature before application. Positive photoresist is a viscous light sensitive liquid polymer used in photolithography that becomes soluble in a photoresist developer solution after being exposed to ultraviolet light. 


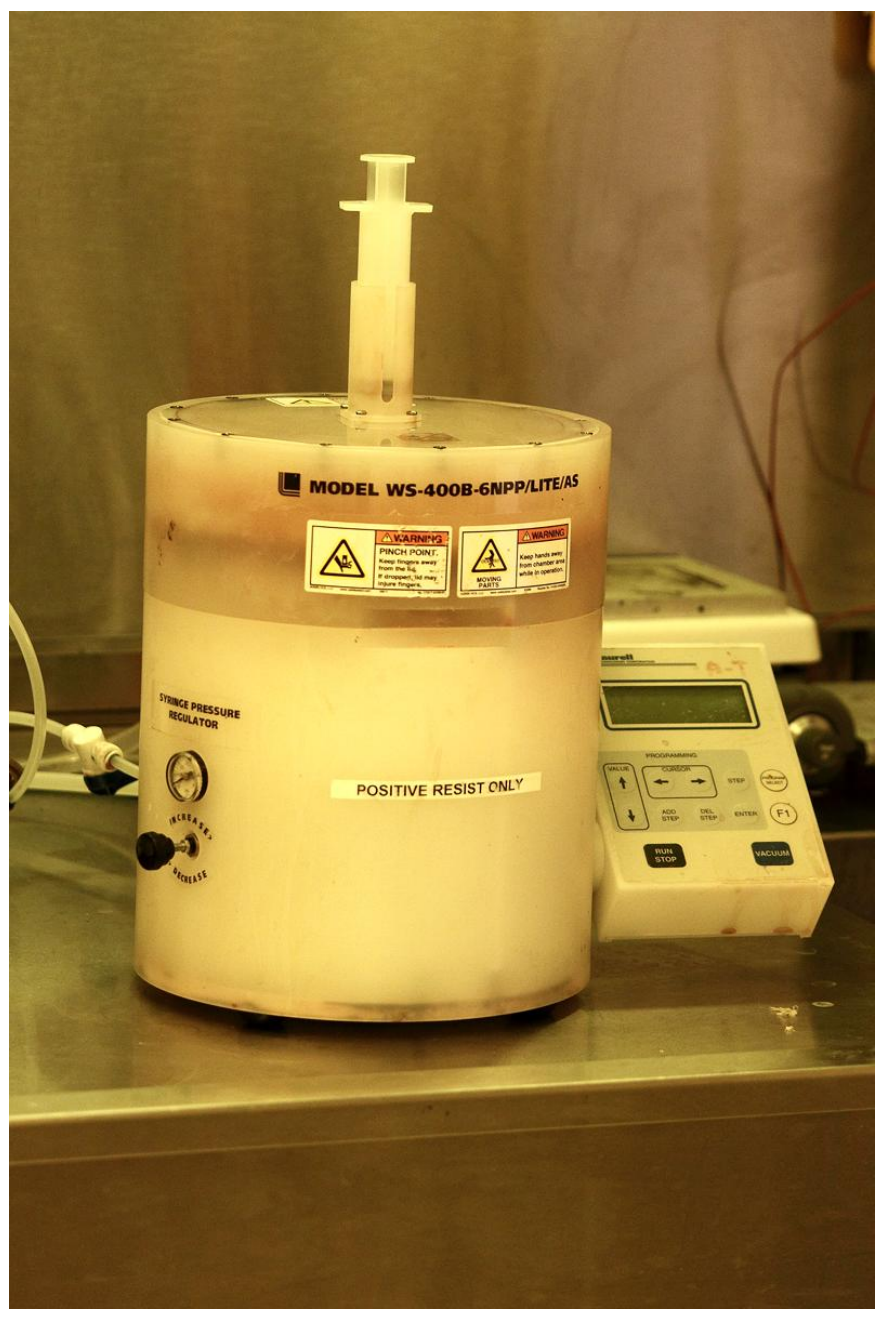

Figure 42 - Positive photoresist was spun onto the silicon wafers using a Laurell Technologies WS-400B6NPP spin coating system.

The silicon wafer was aligned using an alignment tool and then attached to a vacuum chuck in the spin coating chamber. Throughout the spin coating process low purity nitrogen gas was injected into the system. To improve the adhesion of the photoresist to the wafer's surface, about $5 \mathrm{~mL}$ of a primer, hexamethyldisilazane (HMDS), was applied to the wafer and then spun to cause evaporation. This process is called priming the surface and there are typically two methods, liquid priming and vapor priming. HMDS is an adhesion promoter that stops water vapor from adsorbing on the surface of the wafer. If water vapor 
is adsorbed onto the surface then the photoresist will tend to adhere to the water vapor instead of the surface, resulting in poor adhesion. ${ }^{41}$ HMDS gives the best adhesion when it is introduced in its vapor phase. Quickly after the HMDS finished spinning, $3-5 \mathrm{~mL}$ of positive photoresist was manually deposited on the wafer's surface with a syringe through the top of the spin coating chamber. Care was taken to ensure a constant stream of photoresist was deposited because the introduction of air and bubbles onto the surface can cause splotches to appear. An incomplete or poor coating of photoresist will leave locations of the silicon dioxide surface exposed and vulnerable to the wet etching process. The positive photoresist was spun at about 4000 RPM for 20 seconds and then removed from the spin coating vacuum chuck and placed on a hot plate at $90^{\circ} \mathrm{C}$ for 60 seconds to soft bake (Figure 43). Because the oxide on both sides of the wafer needed to be protected from the BOE, the wafer was spin coated with photoresist again on the other side of the wafer and then soft baked.
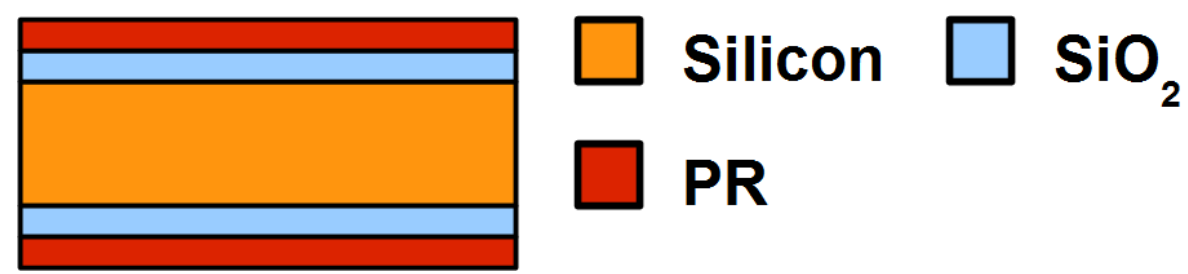

Figure 43 - Positive photoresist was spun onto the surface of the silicon device wafers in order to protect the surface of the oxide from the BOE.

The pre-exposure bake or soft baking process was used to evaporate the solvent from the photoresist. Failure to remove enough of the solvent or baking for too long will affect the resist profile. If baked for too long the photoactive compound deteriorates, thereby reducing the sensitivity of the photoresist. ${ }^{41}$ 
Once the positive photoresist on the wafers was soft baked, it was exposed with UV light provided by a high powered mercury-arc lamp from a Canon PLA501 FA aligner (Figure 44). The UV light was shone through a 5 inch mask above the wafer, exposing only certain locations of the photoresist to the UV light and causing those locations to become soluble in a photoresist developer solution (Figure 45). The time that the photoresist is exposed to the UV light can be changed with a parameter called the light integral, where higher light integrals correspond to longer exposure times. The best looking and most detailed features were found using a light integral of 5.5. Light integrals below 5.5 tended to result in underexposed features and light integrals above 5.5 tended to result in overexposed features.

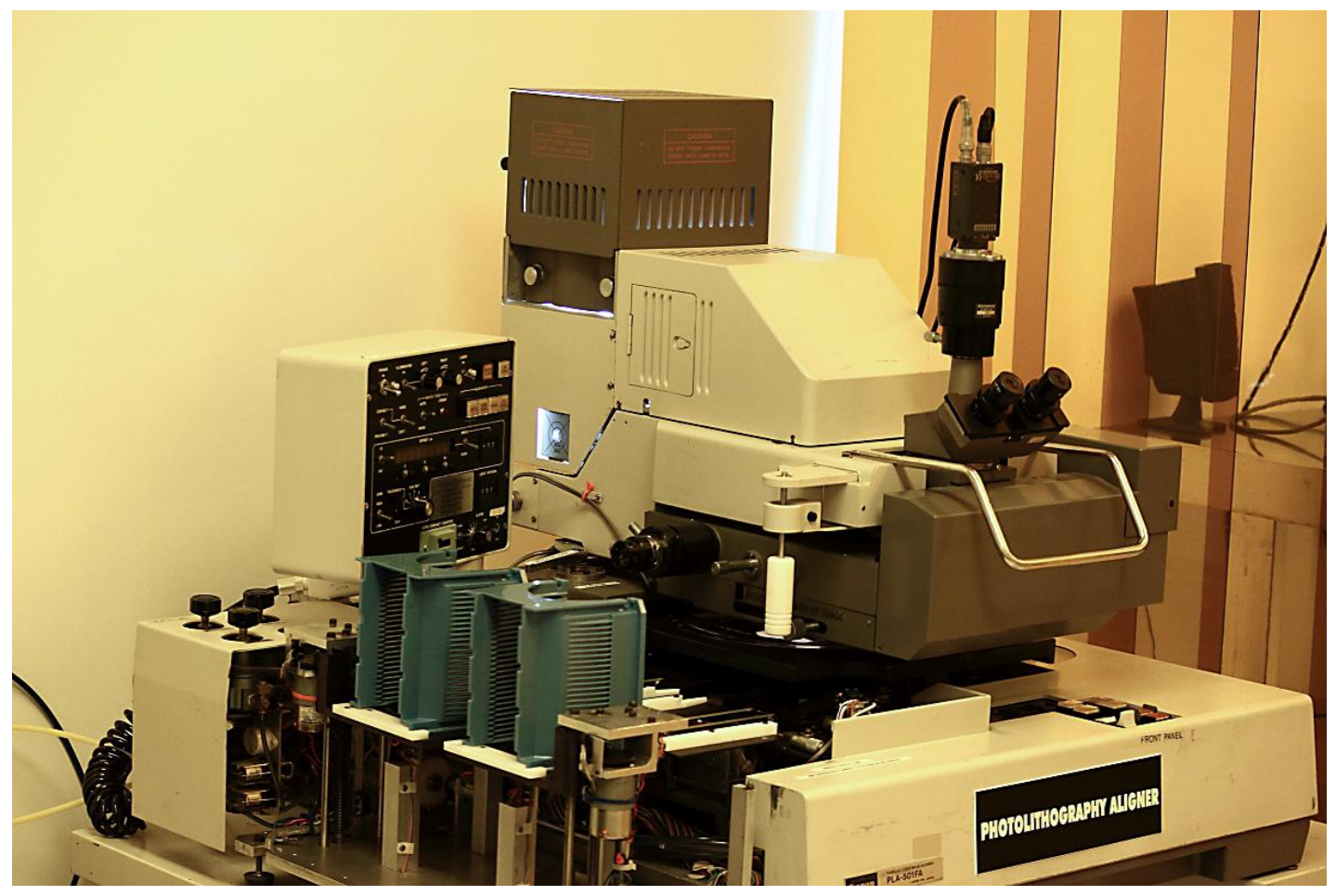

Figure 44 - The positive photoresist was masked and exposed to UV light using a Canon PLA-501 FA aligner with a light integral of 5.5 . 


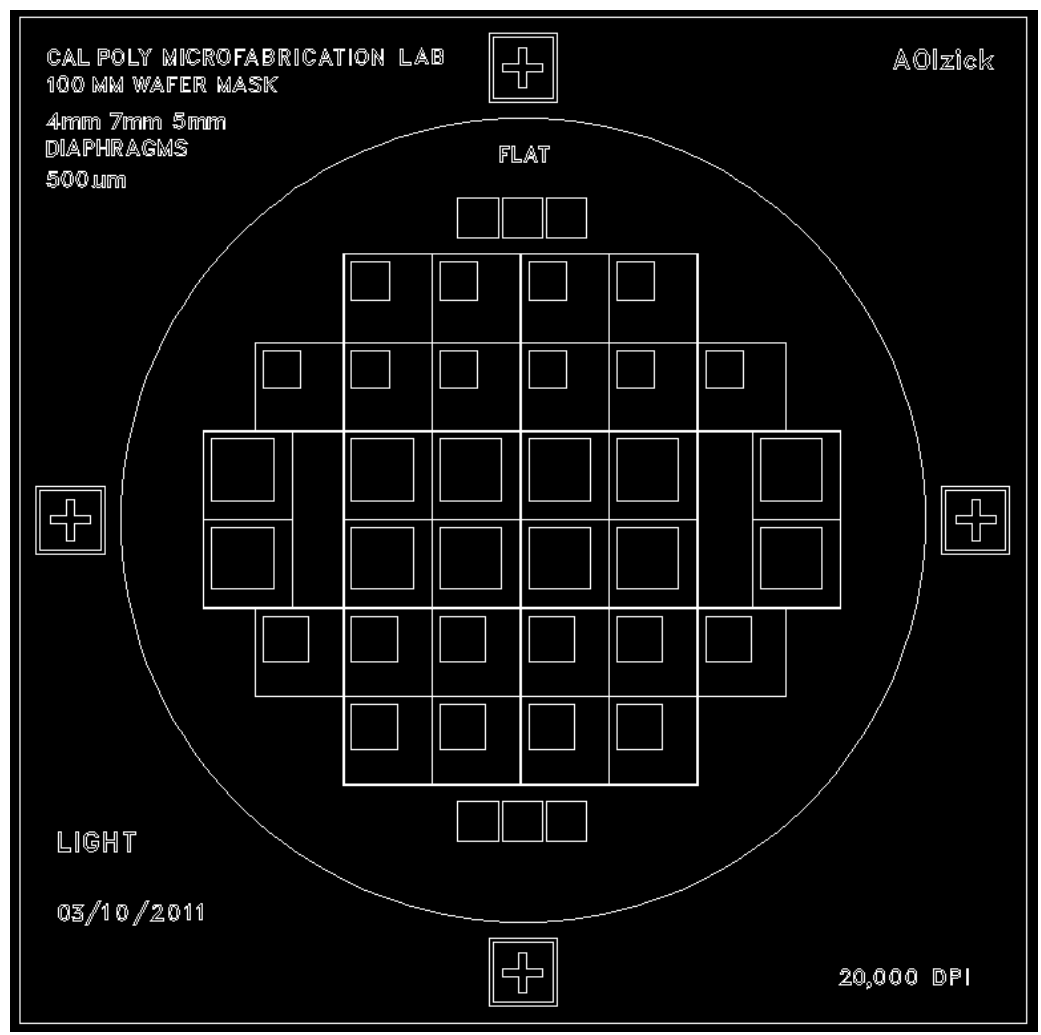

Figure 45 - To etch the microspeaker diaphragms in the silicon, the follow mask was used to pattern the surface. The mask features $4 \mathrm{~mm}, 5 \mathrm{~mm}$, and $7 \mathrm{~mm}$ sized squares surrounded by scribing channels.

Once the photoresist was exposed to the aligner's UV light, the wafer was placed in a beaker of Microposit CD-26 Developer solution, which contains approximately $2.5 \%$ TMAH. The photoresist was developed for approximately 10 minutes with periodic agitation or until the smallest features became visible (Figure 46).
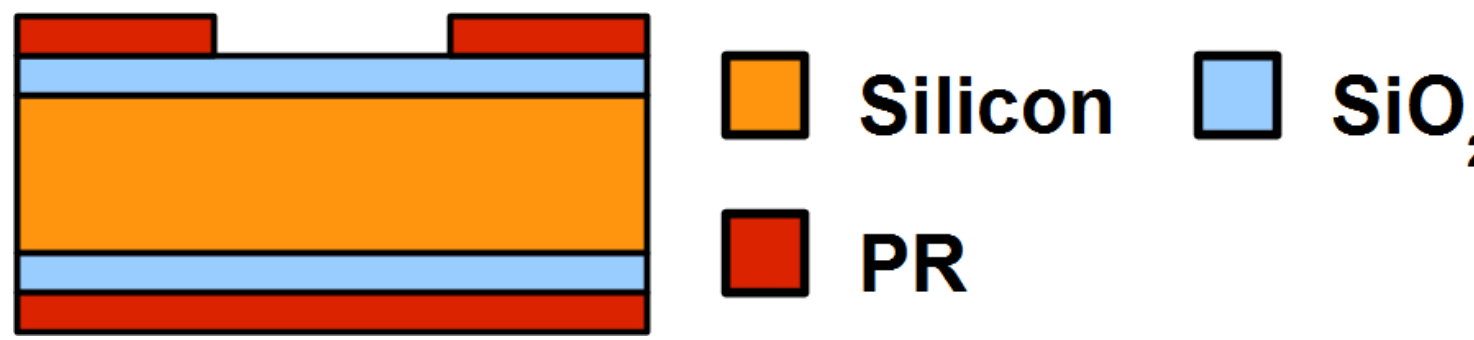

Figure 46 - After being exposed to UV light from the aligner, the photoresist (PR) was developed in a Microposit CD-26 solution for 10 minutes. 
The agitation will wash away old developer and allow fresh developer to come in contact with the photoresist and will result in faster developing rates as well as better defined features. To determine whether the photoresist was properly exposed, the wafer was rinsed with DI water and dried with low-purity nitrogen gas and then examined under an optical microscope. If the features were welldefined and were not overexposed or underexposed then the wafer would be hard baked on a hot plate at $140^{\circ} \mathrm{C}$ for 60 seconds. Performing a hard-bake or post-exposure bake (PEB) will harden the polymer and improve the photoresist's resistance to wet and dry etching, but will also make it more difficult to remove. If patterning for example, channels, overexposure of the photoresist results in channels that are larger than anticipated and underexposing results in channels that are too small (Figure 47). ${ }^{42}$ Underexposing tends to appear when the photoresist is not exposed for a long enough time because the UV light does not reach through the entire film and undergo the chemical reaction to make the resist soluble. Overexposing results in features that are larger than usual because the light at the pattern's edges are scattered and diffracted.

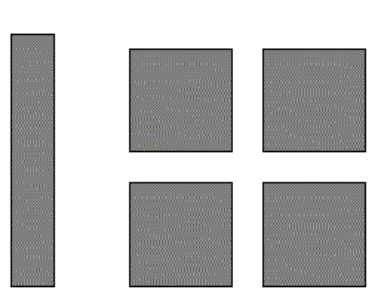

Desired pattern

Resist

Substrate

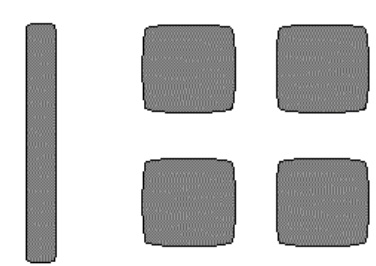

Over-exposed pattem

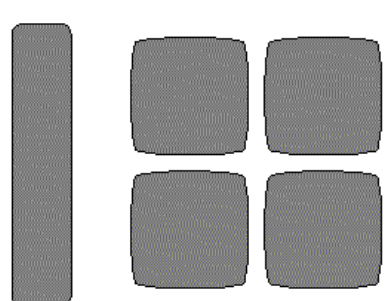

Under-exposed pattem

Figure 47 - Overexposing and underexposing the photoresist can cause undesirable features to appear in the resulting pattern. ${ }^{42}$ 
Once finished cooling after the hard bake, the wafers were placed in room temperature BOE for 7 minutes to etch away the oxide layer (Figure 48). The positive photoresist protected the oxide in certain locations from being etched in the BOE. The etch time was determined by finding the average etch rate of the silicon dioxide in $\mathrm{BOE}$, which was $22.30 \pm 6.34 \AA$ A/second.
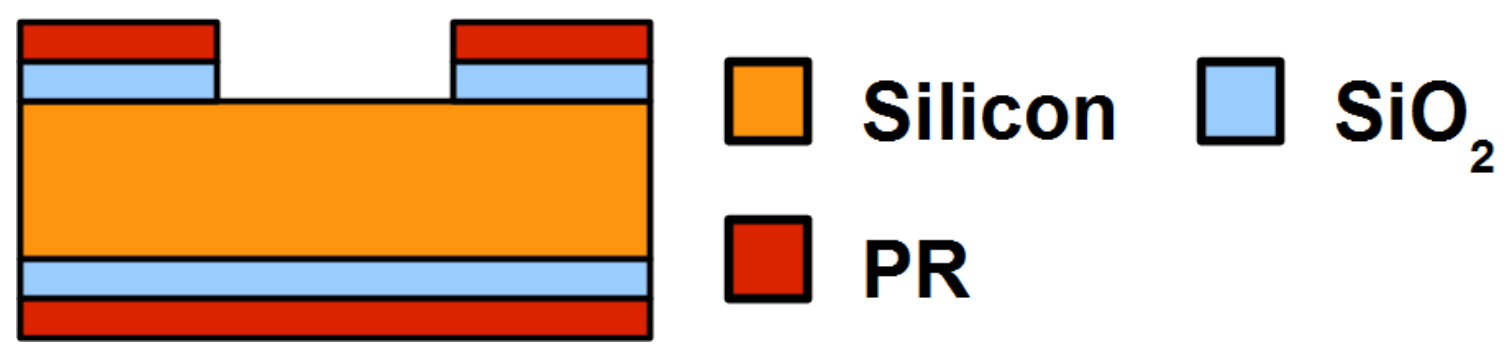

Figure 48 - After the photoresist was developed and hard baked, the silicon dioxide was etched away by BOE for several minutes.

Because the photoresist may contaminate the TMAH silicon wet etching solution and affect the etching quality and etching rate, the photoresist must be removed. The photoresist was removed by immersing the wafers in Microposit Remover 1165 at $60^{\circ} \mathrm{C}$ for approximately 5 minutes or however long it took to remove all of the photoresist (Figure 49). Afterwards, the wafers were cleaned of organic contaminates in a Piranha solution at $70^{\circ} \mathrm{C}$ for 10 minutes.
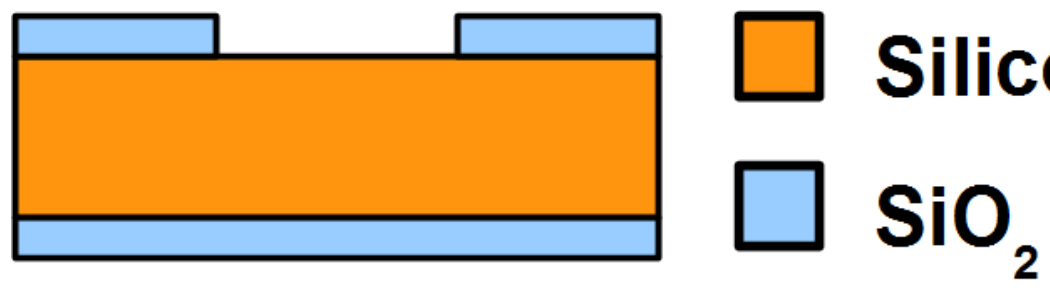

Figure 49 - Before etching the exposed silicon, the photoresist was stripped away using the Microposit Remover 1165 resist stripping solution.

To get the desired diaphragm structure for the microspeakers, the silicon device wafers were deep wet etched in a $25 \%$ solution of TMAH. The TMAH solution was heated to $80^{\circ} \mathrm{C}$ using a hot plate and a resistive heating element 
wrapped around the vessel (Figure 50). The TMAH etchant was stirred with a magnetic stirring bar at the slowest rate possible. To relieve the resistive heating element from heating the entire solution, it was brought up to about $60^{\circ} \mathrm{C}$ with a hot plate and then the resistive heating element was turned on using a temperature controller until it hit $80^{\circ} \mathrm{C}$. Since the temperature of the $\mathrm{TMAH}$ would sometimes overshoot if the resistive heating wrap heated too much, the solution was left until it stabilized at $80^{\circ} \mathrm{C}$.

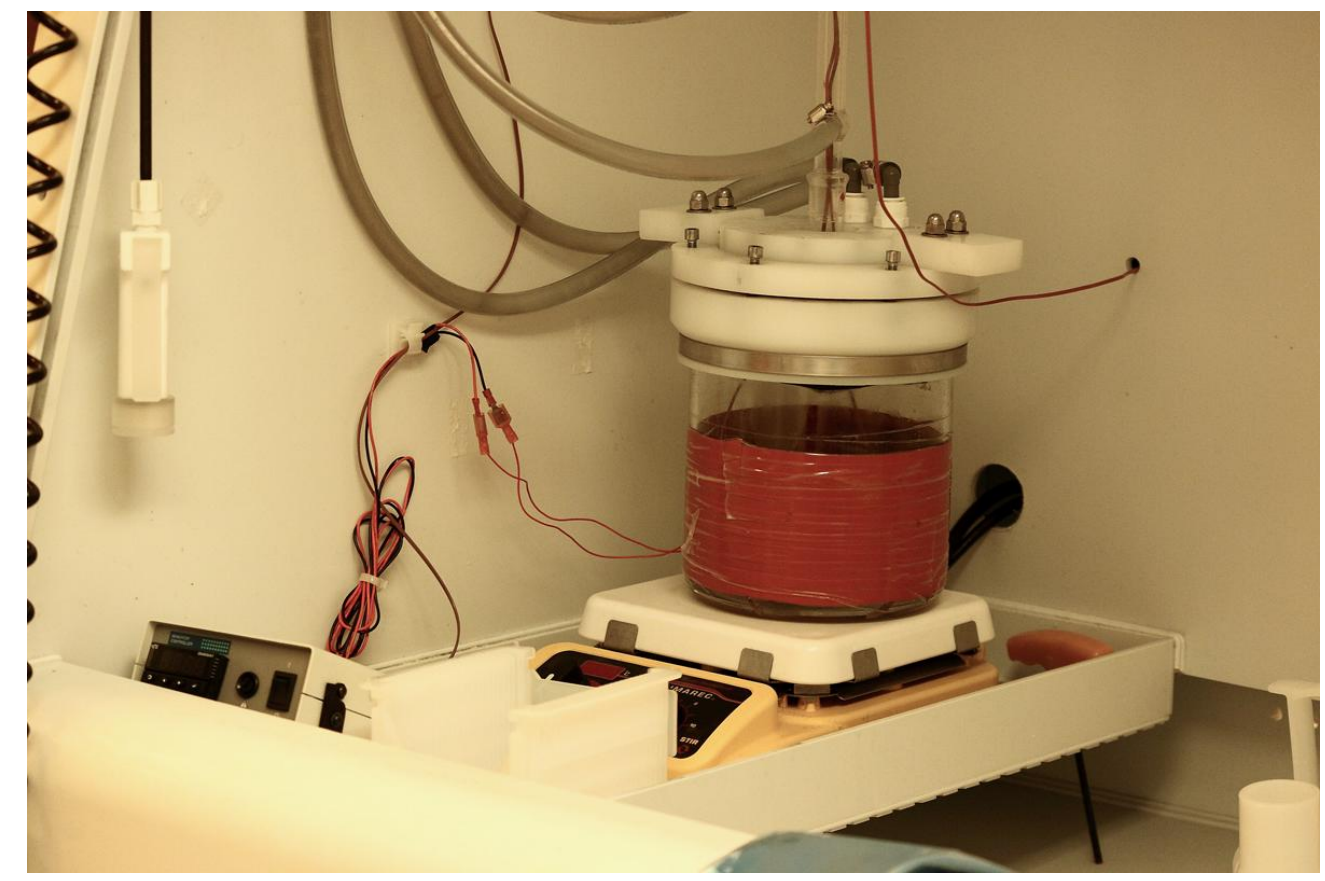

Figure 50 - The $25 \% \mathrm{TMAH}$ silicon wet etching solution was heated to $80^{\circ} \mathrm{C}$ using a resistive heating element and a magnetic stirring hotplate.

The temperature of the solution was monitored with two thermocouples, one for when the hot plate is heating the TMAH and the other for the temperature controller. Because of the number of variables that are involved in the etching process, such as dopant concentration, temperature, stirring speed, etc., the etch rates changed with each wafer. This means that the etch rate $(E R)$ had to be determined for each wafer by performing a short etch at the beginning of each 
wet etch. The etch rate was measured by etching for a few hours and then measuring the depth with a profilometer. However, due to the limits of the profilometer, the depth of the etch could not be measured past $400 \mu \mathrm{m}$. I found that the etch rate for each individual wafer would stay relatively consistent, but would not be the same between wafers. For example, one wafer would have an ER of $22 \mu \mathrm{m} / \mathrm{hr}$ and another would have an ER of $28 \mu \mathrm{m} / \mathrm{hr}$. The total etch time could be calculated because the silicon wafers are about $500 \mu \mathrm{m}$ thick and because the silicon etches anisotropically at an angle of $54.74^{\circ}$ in TMAH. The etching goal was to achieve a $10 \mu \mathrm{m}$ thick silicon diaphragm and it was performed in 5-6 hour increments to allow for the greatest control over the etch depth (Figure 51). The typical etchants used for wet etching silicon are alkali hydroxide based, such as $\mathrm{KOH}$ and $\mathrm{TMAH}$, and they undergo the following chemical reaction ${ }^{43}$ :

$$
\mathrm{Si}+2 \mathrm{OH}^{-}+2 \mathrm{H}_{2} \mathrm{O} \rightarrow \mathrm{Si}(\mathrm{OH})_{2}^{2-}+2 \mathrm{H}_{2}
$$

Unfortunately, the etch rate seems to change at the beginning and end of the etching process, making it difficult to obtain a thin diaphragm without etching through the diaphragm or making it too thick.
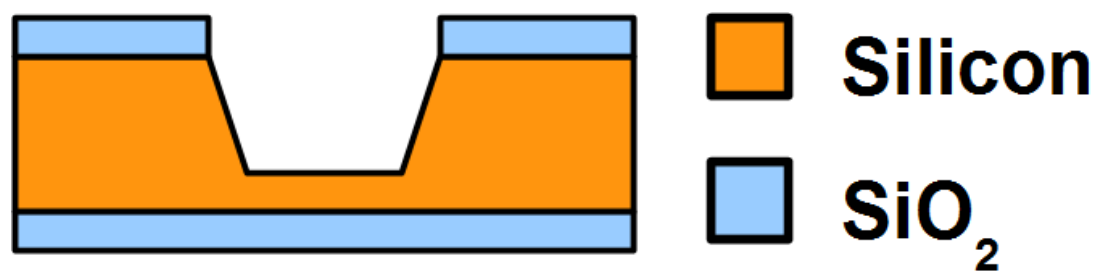

Figure 51 - After deep wet etching the silicon, a thin silicon layer was all that was left. The $\mathrm{ZnO}$ structure was deposited on the other side of the wafer to cause oscillations of the silicon layer.

Unfortunately, the actual thickness of the diaphragm could not be measured with the profilometer so the thickness is only an estimated value. After carefully 
removing the delicate silicon wafers from the TMAH, they were rinsed with DI water, dried with nitrogen, and then examined under an optical microscope (Figure 52).

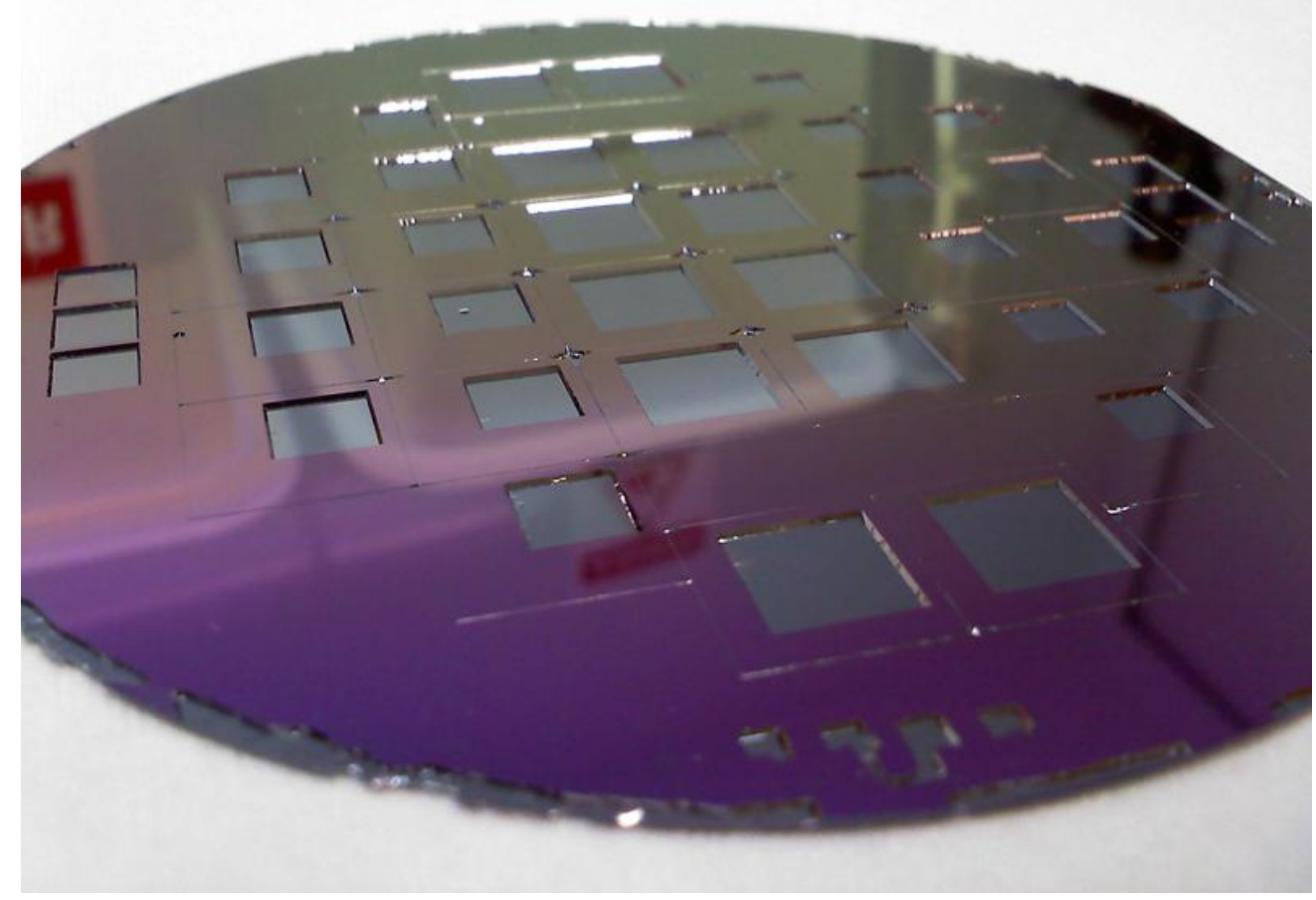

Figure 52 - The silicon device wafers were delicate after deep etching and had to be handled with care. In certain locations around the wafer some of the diaphragms were etched all of the way through.

The next step was to deposit a thin layer of aluminum on the opposite surface to act as the electrode for the $\mathrm{ZnO}$ actuator structure. DC magnetron sputtering was performed on an Advanced Micro Devices Torr CRC-150 physical vapor deposition (PVD) machine at $100 \mathrm{~W}$ and 10 mTorr Ar with a 3 minute presputter and a 30 minute sputter (Figure 53). A 99.99\% pure aluminum target was used to sputter and the chamber was pumped down for about 22 hours. The total pressure of the vaccum chamber was 15 mTorr, but because the base pressure of the chamber was $5 \mathrm{mTorr}$, only $10 \mathrm{mTorr}$ of argon was actually injected into 
the chamber. The aluminum thin film was $711 \mathrm{~nm}$ thick and therefore the sputter rate at $100 \mathrm{~W}$ and $15 \mathrm{mTorr}$ Ar was determined to be $23.7 \mathrm{~nm} / \mathrm{min}$.
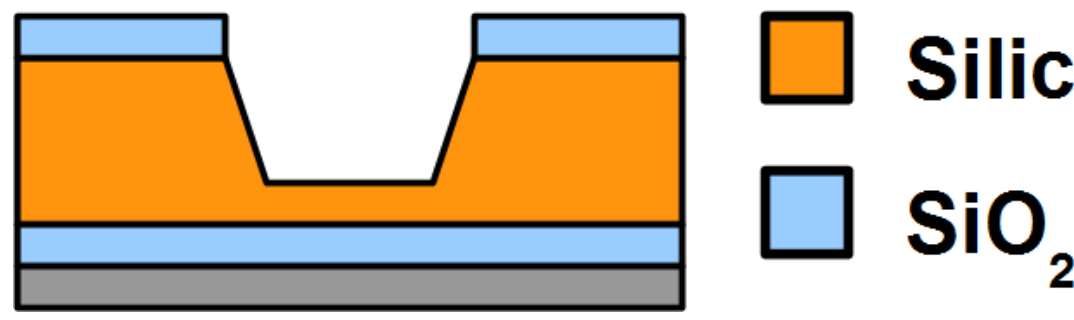

Figure 53 - A thin film of aluminum was deposited onto the silicon wafer to act as the bottom electrode of the $\mathrm{ZnO}$ actuator structure.

Next, the aluminum electrodes were patterned with photoresist. The patterning was performed using the same process as the patterning for the silicon etching, except for a few changes that were required because of the etched backside of the wafer. Because of the etched diaphragms, the vacuum chuck on the spin coater would not hold onto the wafer well enough or would cause the wafer to break. To accommodate the diaphragms and allow spin coating, carefully shaped aluminum foil was taped to the bottom of the silicon wafer (Figure 54). After spin coating and soft baking the photoresist, the aluminum foil was removed and the photolithography process continued normally, starting with alignment, patterning, and developing (Figure 55). 


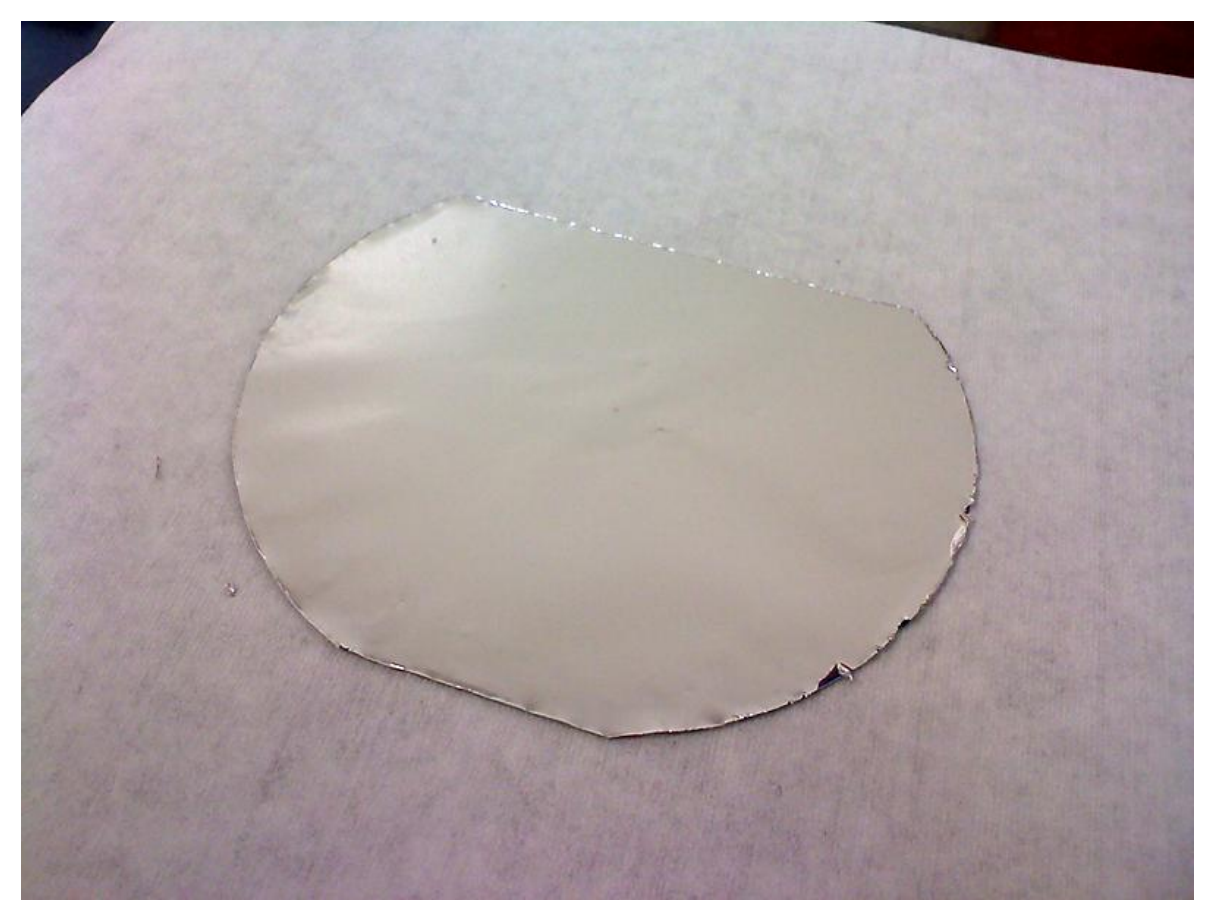

Figure 54 - To spin coat more photoresist onto the wafer for lithography of the deposited aluminum electrodes, aluminum foil had to be taped to the backside of the wafer.

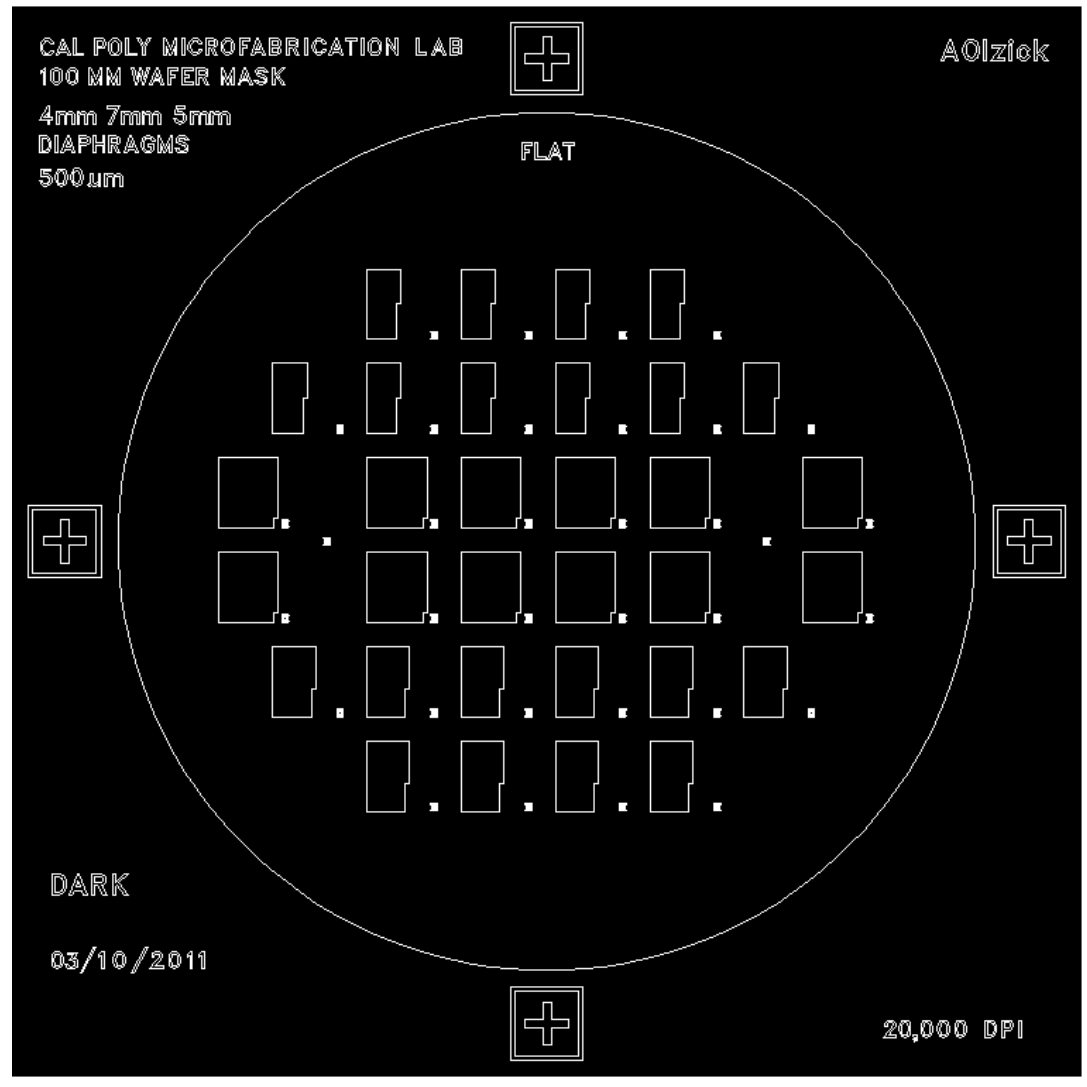

Figure 55 - The first layer of the $\mathrm{ZnO}$ actuator structure consisted of patterned aluminum electrodes that would provide electrical access to the bottom of the $\mathrm{ZnO}$ film. 
After patterning the photoresist, the aluminum electrodes were etched away with Transene Aluminum Etchant Type $A$ at $50^{\circ} \mathrm{C}$. The etchant is an aqueous solution containing acetic, nitric, and phosphoric acid and does not etch $\mathrm{Si}, \mathrm{SiO}_{2}$, or positive photoresists. The etchant's etch rate is approximately $100 \AA / \mathrm{sec}$ and therefore the $711 \mathrm{~nm}$ thick aluminum film was etched for about 1 minute and 30 seconds (Figure 56).
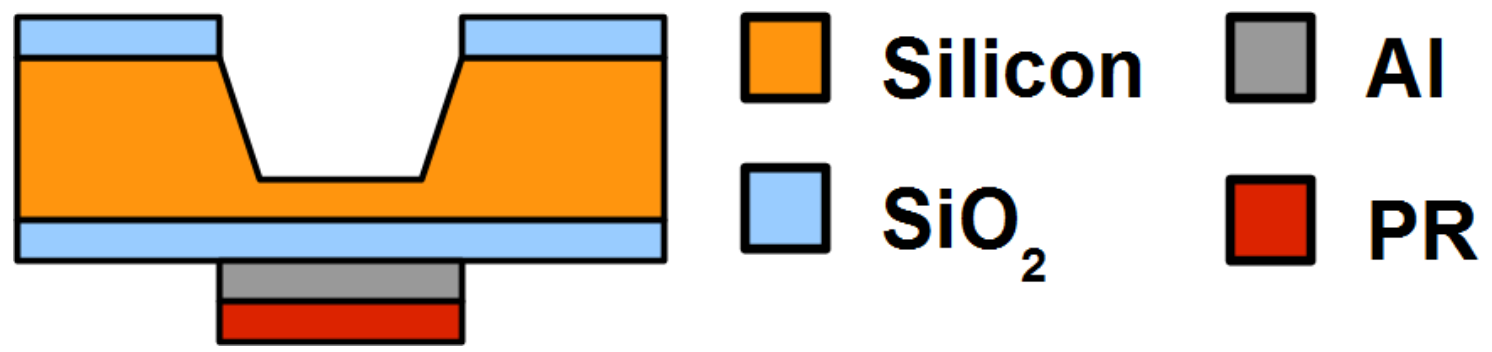

Figure 56 - After sputtering aluminum on the silicon wafers, photoresist was patterned on top of the aluminum and then the aluminum was etched away with an aluminum etchant.

After etching the aluminum, the photoresist was stripped away and $\mathrm{ZnO}$ was deposited onto the surface. Unfortunately, not many device wafers survived up to this point and only a 1:1 oxygen to argon gas ratio was attempted. In addition, at this stage in the thesis the main focus was to fabricate a device and to determine the processing steps and its feasibility, not to extensively characterize its properties. The $\mathrm{ZnO}$ was reactively $\mathrm{DC}$ magnetron sputtered at $100 \mathrm{~W}$ for 45 minutes with a 15 minute pre-sputter and with argon and oxygen partial pressures both at $11 \mathrm{mTorr}$. Since this thesis evolved into more of a characterization of $\mathrm{ZnO}$, a more detailed description of the PVD process will be described later in the characterization section.

After the $\mathrm{ZnO}$ deposition was performed, the $\mathrm{ZnO}$ was patterned with positive photoresist with the same process as was used on the aluminum thin film (Figure 
57). However, instead of an aluminum etchant, a $\mathrm{ZnO}$ etchant of acetic acid and DI water in a 1:30 ratio was used to etch away the excess $\mathrm{ZnO}$ (Figure 58).

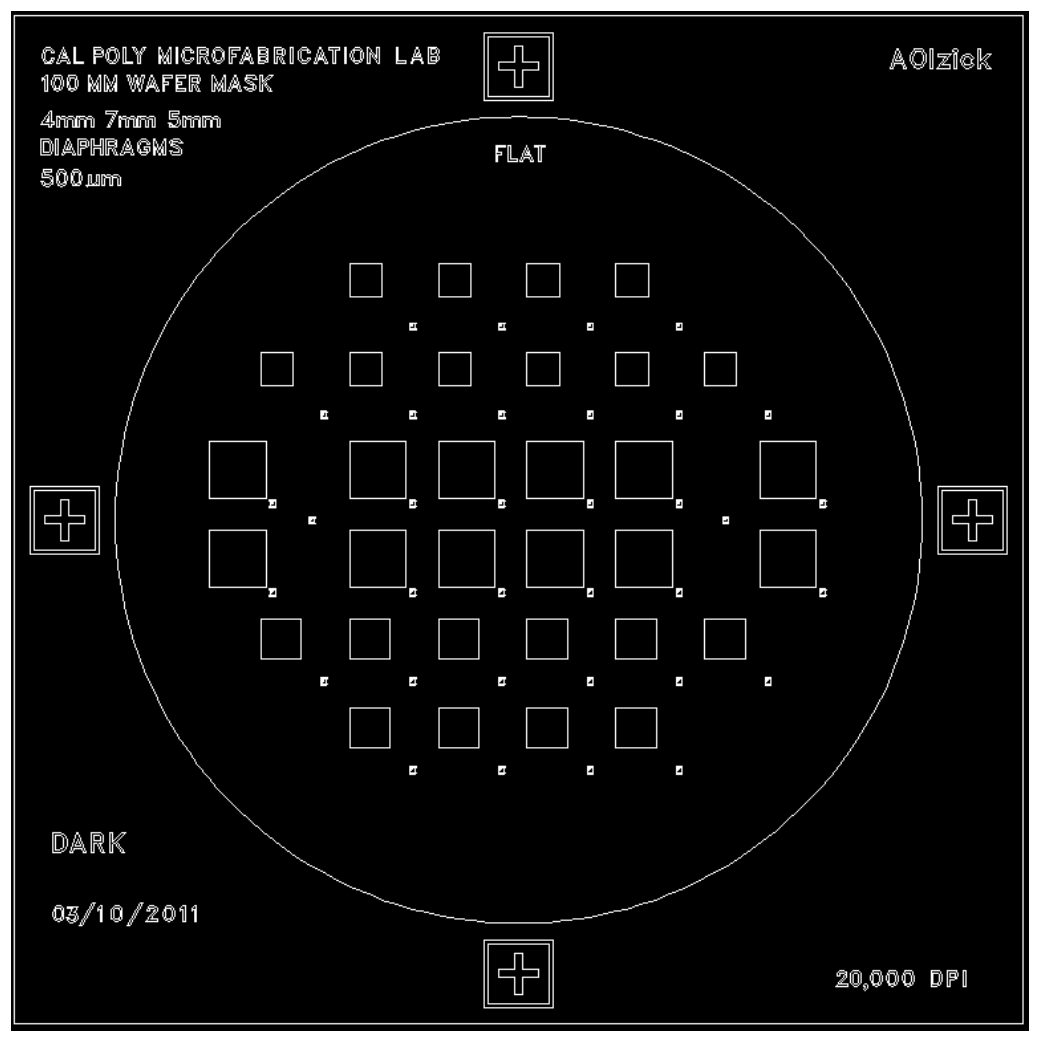

Figure 57 - The ZnO was patterned with photoresist into $4 \mathrm{~mm}, 5 \mathrm{~mm}$, and $7 \mathrm{~mm}$ squares directly over the diaphragm structure.
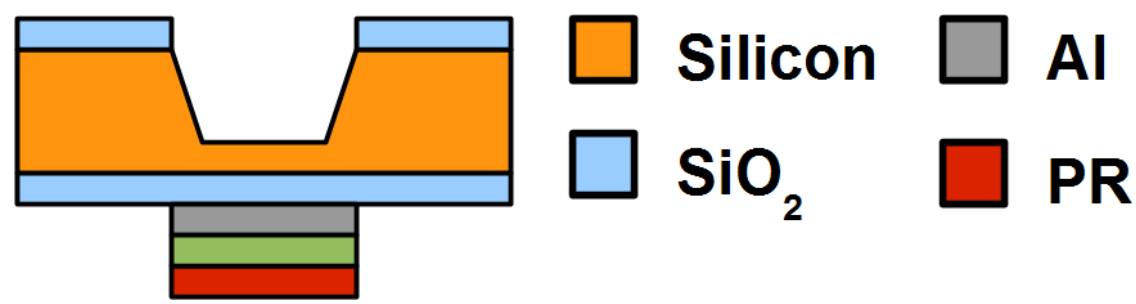

Figure 58 - After depositing $\mathrm{ZnO}$, spin coating on photoresist, and patterning the photoresist, the $\mathrm{ZnO}$ was etched away with a weak acetic acid aqueous solution.

The positive photoresist was stripped away again using Microposit Remover 1165 and then one more layer of aluminum was sputtered onto the $\mathrm{ZnO}$ to finish the actuator structure. The aluminum was sputtered with the same parameters as before, which was a 3 minute pre-sputter followed by a 30 minute sputter at 100 
watts with a 10 mTorr argon pressure. Then positive photoresist was spun onto the wafer's surface, exposed in the aligner, developed in Microposit CD-26, and then hard baked at $140^{\circ} \mathrm{C}$ for 60 seconds. The resulting photoresist protected the second aluminum electrodes from the aluminum etchant and created an electrical contact (Figure 59). Once the aluminum etching was complete, the photoresist was stripped, leaving only the $\mathrm{ZnO}$ actuator structure with electrical contacts (Figure 60).

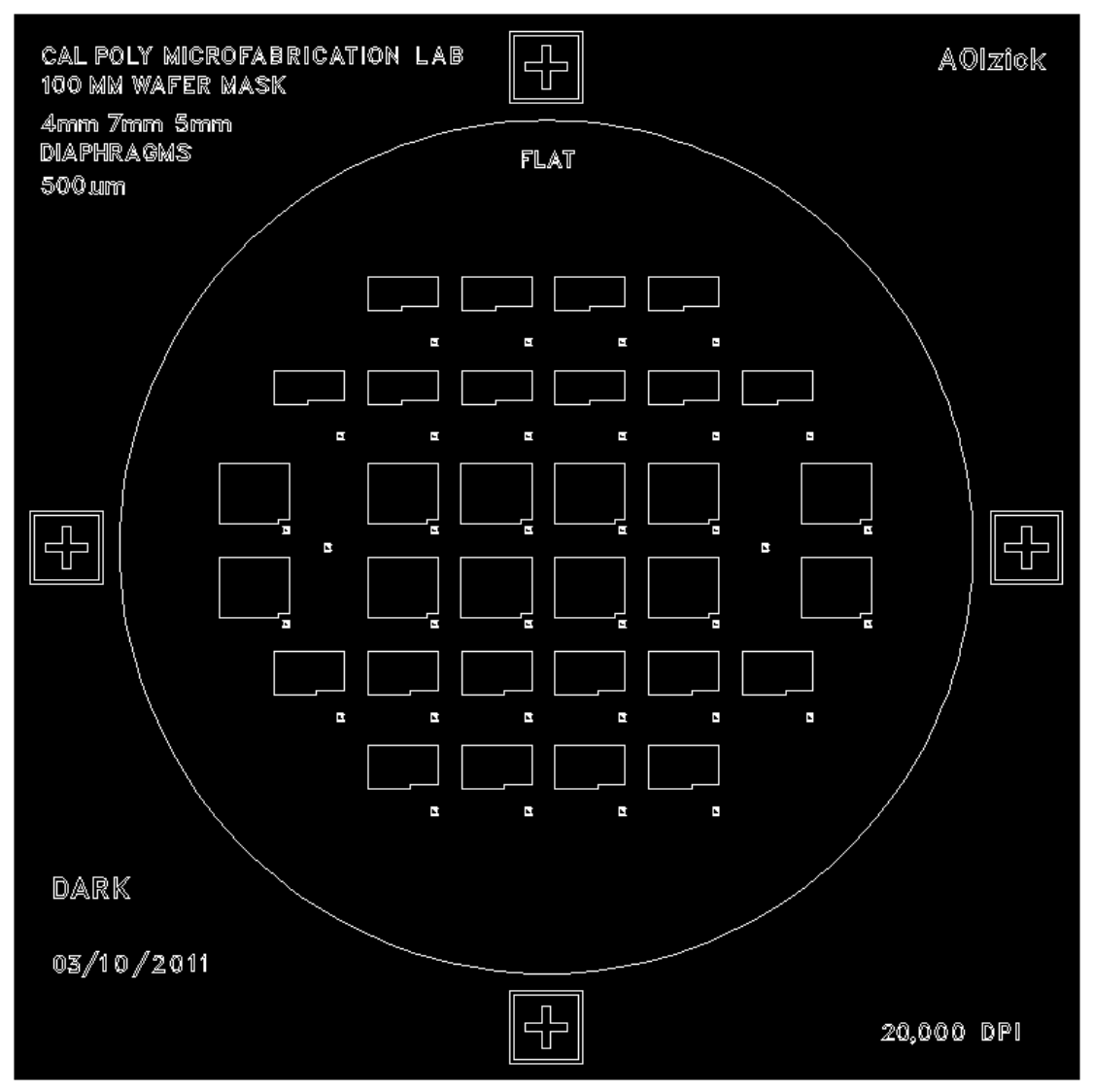

Figure 59 - The last layer of the structure was an aluminum layer that acted as the top electrode of the microspeaker. This layer is similar to the first aluminum electrode layer, except oriented in another direction to avoid shorting with the first aluminum layer. 


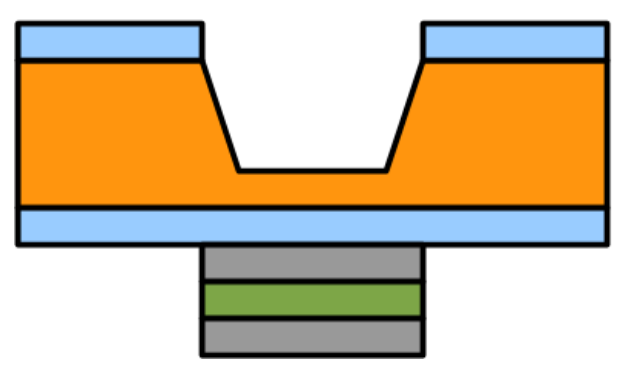

$\square$ Silicon $\square$ Al

$\square \mathrm{SiO}_{2}$

$\square \mathrm{ZnO}$

Figure 60 - The complete microspeaker structure was obtained after sputtering and etching the last layer of aluminum.

Once the complete structure was finished, each device was broken off of the wafer and tested by attaching the top and bottom electrodes to a function generator (Figure 61). The function generator provided up to $10 \mathrm{~V}_{\mathrm{p}-\mathrm{p}}$ of alternating current at varying frequencies from one hertz to hundreds of kilohertz. Because I was unsure whether $10 \mathrm{~V}_{\mathrm{p}-\mathrm{p}}$ was enough voltage to actuate the $\mathrm{ZnO}$, the function generator was also attached to a power amplifier that could amplify the $A C$ voltage to hundreds of volts. However, the device still produced no sound.

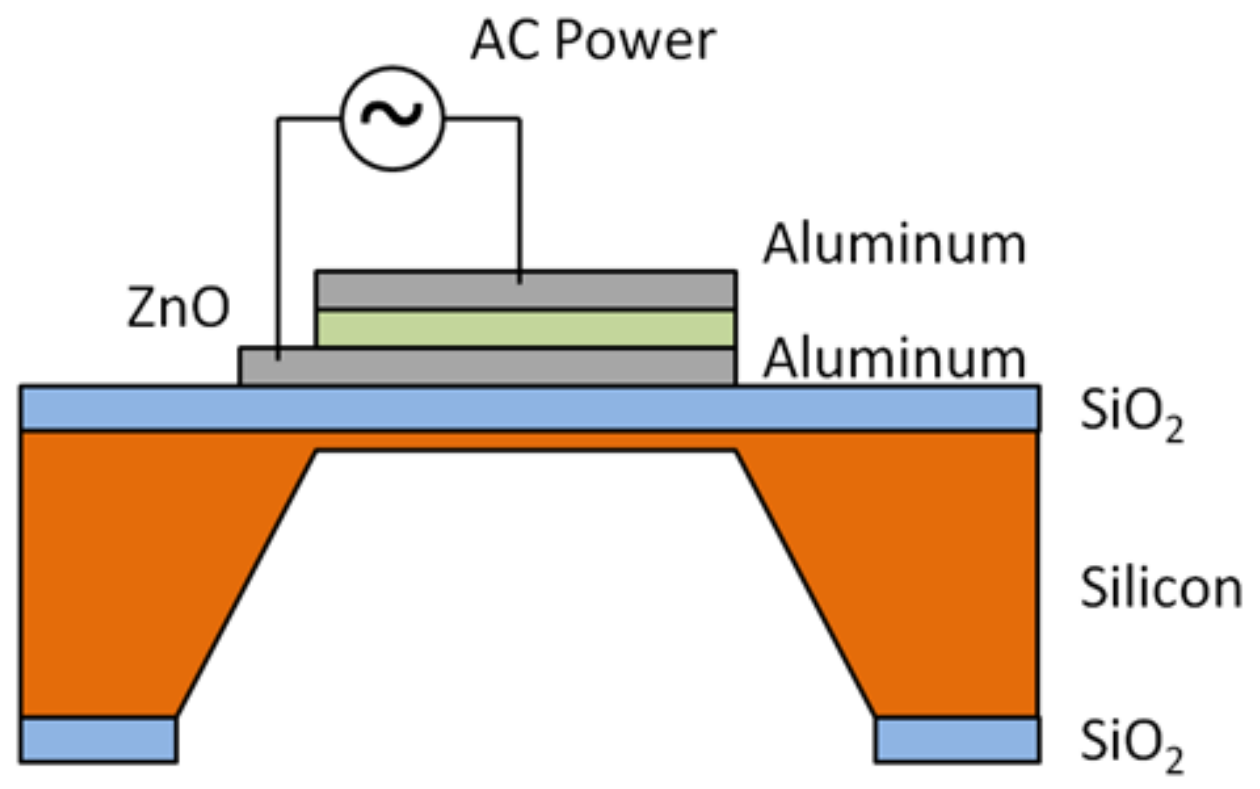

Figure 61 - In order to create a microspeaker, the $\mathrm{ZnO}$ thin film must be able to oscillate a thin silicon diaphragm that was etched into a $100 \mathrm{~mm}$ n-type single crystalline silicon wafer. 


\subsection{Characterization}

Characterization of the $\mathrm{ZnO}$ thin films were completed using the techniques outlined in section 1.5 to evaluate the structural, optical, electrical, and piezoelectric properties of the films. Each process will be thoroughly explained so that future students may reference this document for guidance.

\subsubsection{Physical Vapor Deposition (PVD)}

For the characterization portion of the thesis, $\mathrm{ZnO}$ thin films were deposited onto n-type silicon wafers using physical vapor deposition (PVD) on an Advanced Micro Devices (AMD) Torr CRC-150 sputtering system (Figure 62). The "Torr" sputtering system was connected to a DC Plasma Generator and the plasma gases were controlled using a custom built gas flow controller. Ultra-high purity argon and high-purity oxygen gas were used to ignite the plasma in the glass vacuum chamber. A 99.99\% pure zinc target was used as the cathode, which was attached to the water chilled top plate of the sputtering system using thermally conductive paste. The sputtering target was held in with a stainless steel cathode holder and was shielded from the sputtering material with a stainless steel cathode shield. An adjustable stainless steel shutter blocks the sputtering target and sputtered material from hitting the substrate during the presputtering phase of the sputtering process. 


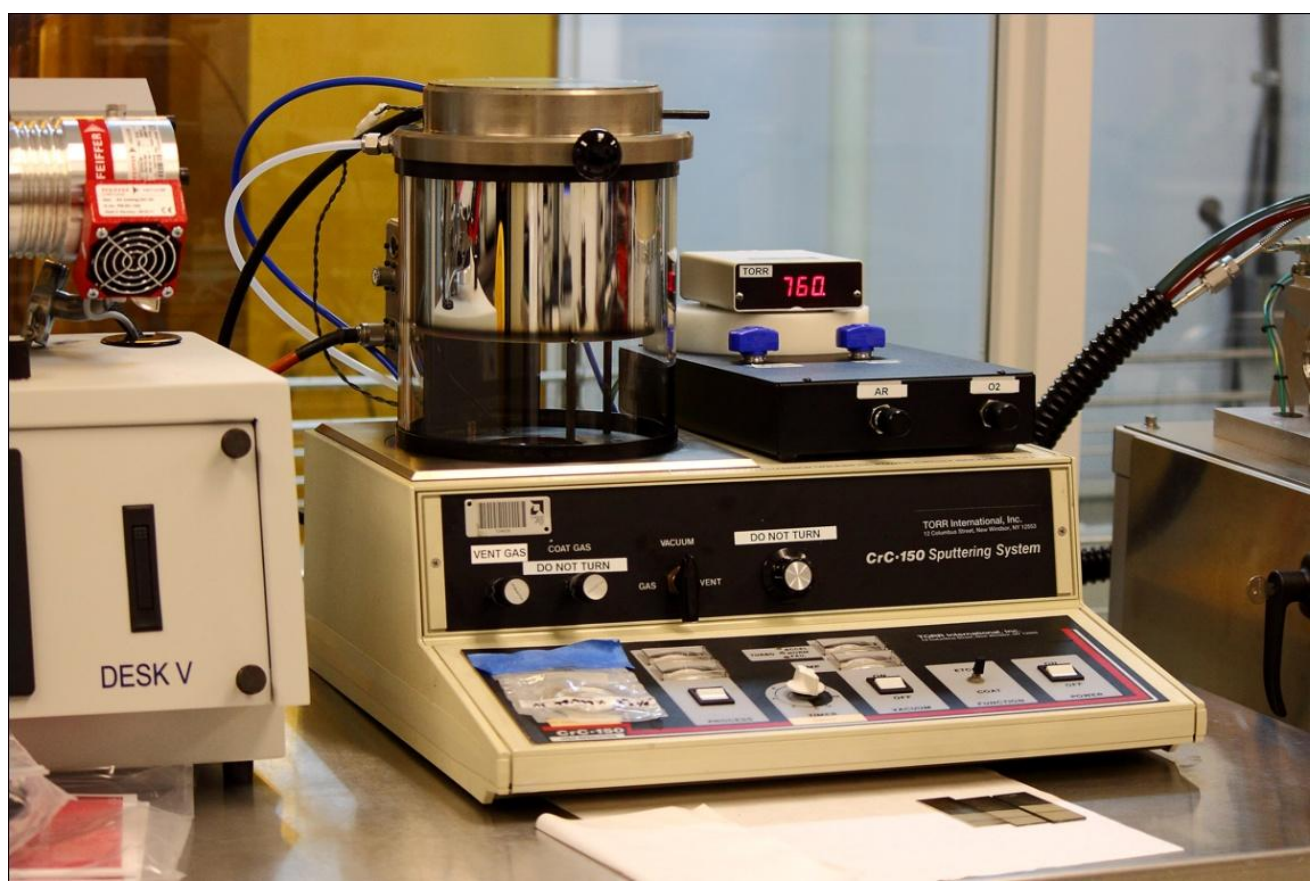

Figure 62 - Physical vapor deposition of aluminum and zinc oxide thin films is performed using the Torr CRC-150. Zinc oxide is deposited by flowing high purity oxygen gas into the chamber using the gas flow controller on the right.

For characterization, the samples were typically pre-sputtered with the shutter closed for 15 minutes and then sputtered with the shutter open for either 30,45 , or 60 minutes. The DC sputtering power was varied between 100 watts and 70 watts. The gas ratio of argon to oxygen $\left(\mathrm{Ar}: \mathrm{O}_{2}\right)$ was varied between a 2:1, 1:1, $1: 2$, and 1:3 gas ratio. Each gas ratio was sputtered at the two power levels (70, $100 \mathrm{~W})$ and the three sputtering times $(30,45,60$ mins.), resulting in a total of 24 $\mathrm{ZnO}$ samples. In order to conserve the amount of silicon wafers utilized in this characterization process, the $\mathrm{ZnO}$ films were sputtered onto diced pieces of $\mathrm{n}$ type silicon wafers that were ultrasonically cleaned for 15 minutes in acetone, rinsed in isopropyl alcohol, rinsed in deionized water, and then dried with low purity nitrogen (Figure 63). 


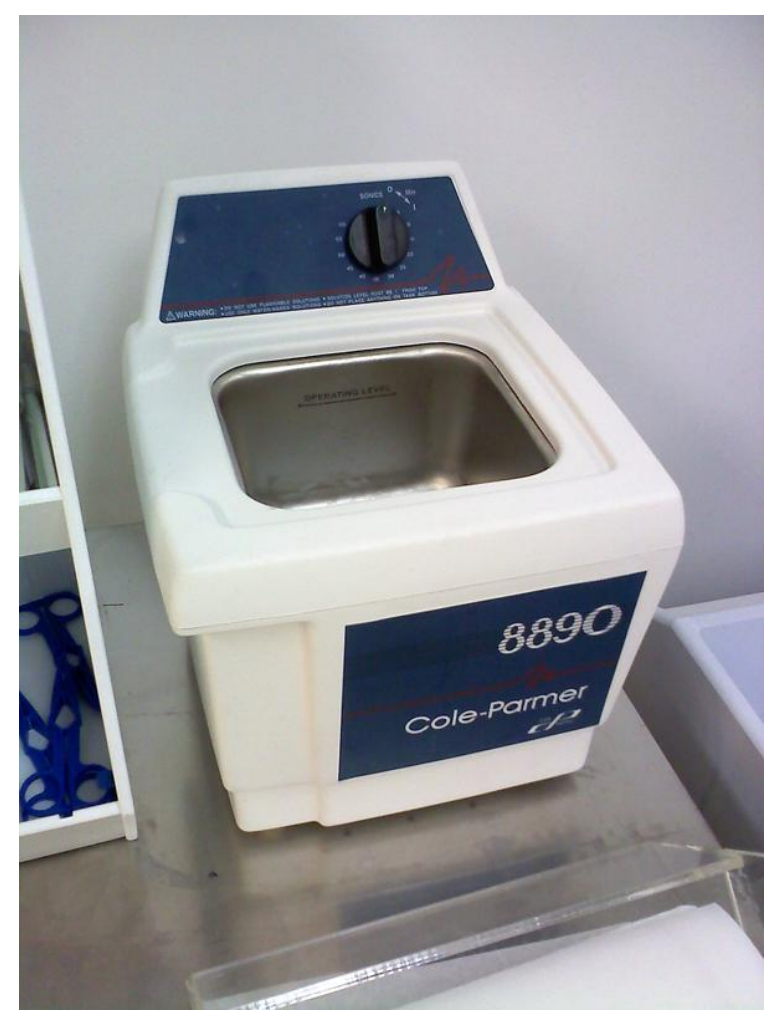

Figure 63 - The diced pieces of n-type silicon were ultrasonically cleaned for 15 minutes in an acetone bath and then rinsed with isopropyl alcohol, DI water, and then dried with nitrogen.

Before sputtering, I would ensure that the chamber, the cathode shield, and the cathode holder were clean. If the glass chamber had another type of metal deposited onto it, such as aluminum, I would put the chamber in a ceramic dish and clean it in Piranha at $70^{\circ} \mathrm{C}$ until the aluminum was completely dissolved in the Piranha. If chromium was on the chamber, a chromium etchant would have to be used. The chamber would then be rinsed with DI water and dried with lint-free clean room wipes, IPA, and nitrogen. A very thin layer of vacuum grease would be applied to the top and bottom of the chamber where the O-rings sit. A thin layer of vacuum grease would also be applied to the inner parts of the O-rings. When the O-rings are placed on top of the chamber, another thin layer of vacuum grease is applied on top of the O-rings. The samples were placed into the chamber and then the power to the Torr was turned on and a vacuum pulled for 
at least an hour. A mechanical roughing pump is used to get the system to around 1 Torr and then a turbo-molecular pump automatically turns on and gets the chamber to a base pressure of 4-5 mTorr. After pumping down, the ultra-high purity argon and high purity oxygen were turned on. In order to achieve the desired gas ratios, the argon gas tank pressure was increased to about 55-60 psi. The system was then turned from Vacuum to Gas. Because the flow is controlled by the gas flow controller, no gas has entered the chamber yet. The argon valve on the flow controller was turned on and allowed to stabilize until it reached the desired partial pressure, making sure to account for the 4-5 mTorr base pressure. As an example, a 1:1 gas ratio will be set, which means 15 mTorr of $\mathrm{Ar}$ and $15 \mathrm{mTorr}$ of $\mathrm{O}_{2}$. The flow is controlled with knobs on the front of the flow controller. After the argon stabilized to about 20 mTorr, the oxygen was turned on until the total pressure reached $35 \mathrm{mTorr}$. Once the pressure stabilized again, the sputtering power would be turned on and the plasma ignited.

\subsubsection{Annealing}

Annealing $\mathrm{ZnO}$ can be a useful method to improve the alignment and orientation of the $\mathrm{ZnO}$ crystals, can increase the size of the crystals, increase the electrical conductivity, and improve the piezoelectric response of the $\mathrm{ZnO}$. Annealing of $\mathrm{ZnO}$ was performed at varying temperatures and times to identify which annealing temperature and time resulted in the best piezoelectric response. The $\mathrm{ZnO}$ thin films were deposited onto $\mathrm{n}$-type (100) single crystalline silicon wafers and samples were annealed at $600^{\circ} \mathrm{C}, 800^{\circ} \mathrm{C}$, and $1000^{\circ} \mathrm{C}$ in a high-purity nitrogen environment. In addition, a control wafer that underwent no 
annealing was included in the analysis. The $\mathrm{ZnO}$ thin films were annealed for thirty minutes at each temperature and at $800^{\circ} \mathrm{C}$ for one hour to see the effects of increasing annealing time. SEM and AFM images of the $\mathrm{ZnO}$ surface were taken after annealing to determine the effect of the annealing on its structural properties. XRD was also performed on the films to determine how annealing affected the orientation of the $\mathrm{ZnO}$ crystals.

Before deposition of $\mathrm{ZnO}$, the silicon wafer substrates were first cleaned of organic contaminates by placing the wafers in a Teflon boat and then immersing them in a piranha solution at $70^{\circ} \mathrm{C}$ for ten minutes. Afterwards, the wafers were rinsed with deionized water and then immersed in a BOE solution at room temperature that would remove any residual oxides from the surface. The silicon wafers were then rinsed again with deionized water and then dried with low purity nitrogen gas. Once the wafers were clean and dry, they were placed in the sputtering chamber of the AMD Torr CRC-150 sputtering machine. Kapton tape was placed over the wafers in order to keep the wafer in place and to obtain a step height that could be used later to measure the thickness of the $\mathrm{ZnO}$ thin film with a profilometer. Next, the chamber was closed and the chamber was pumped for approximately 1 hour to a base pressure of $5 \mathrm{mTorr}$. After the $5 \mathrm{mTorr}$ base pressure was reached, 15mTorr of ultra-high purity argon gas was introduced into the chamber, resulting in a total chamber pressure of 20 mTorr. Next, $15 \mathrm{mTorr}$ of high purity oxygen gas was introduced into the $20 \mathrm{mTorr}$ chamber to act as the reactive gas during the sputtering process. The final sputtering pressure was 35 mTorr, which results from the 15 mTorr of argon and oxygen 
and the $5 \mathrm{mTorr}$ base pressure. The DC plasma generator was turned on and set to a power of $100 \mathrm{~W}$ and the shutter was placed over the $\mathrm{Zn}$ target for a 5 minute pre-sputter to remove contaminates from the surface. After 5 minutes, the shutter was moved, allowing the $\mathrm{ZnO}$ to deposit onto the silicon substrate. $\mathrm{ZnO}$ was sputtered on the substrate for 90 minutes. The films were deposited at room temperature, although some heating of the substrate did occur during the sputtering process.

After sputtering, the chamber was vented and the samples were removed. The Kapton tape on the surface was removed and the residual adhesive on the surface of the wafer was cleaned with acetone and a cotton swab. The film thickness was then measured using an Ambios XP-1 profilometer and the step height that was created from the Kapton tape. After the thickness was measured in several locations across the wafer, they were placed in a quartz boat and were prepared for the thermal oxidation furnace. Two "dummy" wafers were placed on each side of the $\mathrm{ZnO}$ wafer to ensure that the $\mathrm{ZnO}$ wafer received an even amount of heat from the furnace and did not receive any drafts of heat that could cause a non-uniformly heated surface. For the $600^{\circ} \mathrm{C}$ anneal, once the middle of the furnace tube in the thermal oxidation furnace reached $400^{\circ} \mathrm{C}$, high purity $\mathrm{N}_{2}$ at around 25 psi was pumped into the quartz tube and then the quartz boat was placed inside and slowly moved to the center of the tube. The temperature was increased to $600^{\circ} \mathrm{C}$ and then left in the furnace for 30 minutes. After 30 minutes, the furnace's temperature controller was shut off, but the high purity $\mathrm{N}_{2}$ was left on until the furnace reached about $400^{\circ} \mathrm{C}$ in order to prevent any oxidation of the 
surface of the $\mathrm{ZnO}$ coated wafer. The wafers were then left to air cool overnight. The backside of the wafers were scribed with a glass scribe and diced into small square sections and mounted onto $10 \mathrm{~mm}$ slotted mounts and flat mounts for examination of the cross section and topography in a JOEL JSM-6390 scanning electronic microscope. Imaging was performed on all of the annealed samples and the as-sputtered sample. This annealing process was performed for the rest of the annealing temperatures and times.

\subsubsection{Structural Properties}

The structural properties of the $\mathrm{ZnO}$ thin films were characterized using scanning electron microscopy (SEM), x-ray diffraction (XRD), and stylus profilometry.

\subsubsection{Scanning Electron Microscopy (SEM)}

Cross sectional and topographical images of the $\mathrm{ZnO}$ surface were taken with a JOEL JSM-6390 scanning electron microscope (Figure 64). This SEM has both high vacuum and energy-dispersive $\mathrm{X}$-ray spectrometry capabilities at accelerating voltages between 0.5 and $30 \mathrm{kV}$. The SEM generates its electron beam through a pre-centered tungsten hairpin filament and is capable of imaging at $3.0 \mathrm{~nm}$ resolutions at $30 \mathrm{kV}, 8 \mathrm{~nm}$ at $3 \mathrm{kV}$, and $15 \mathrm{~nm}$ at $1 \mathrm{kV}$. Chamber pressures of $3.0 \times 10^{-6}$ Torr are achieved through a rough mechanical pump and a diffusion pump. The chamber pressure is monitored with an ionization gauge that is attached to the chamber. During imaging, the ionization gauge is disconnected because it can result in electronic and thermal noise in the images and degrade image resolution and quality. Topographical and cross-sectional 
images were taken at 40,000X magnification at working distances (WD) between $8 \mathrm{~mm}$ and $10 \mathrm{~mm}$. Moderate resolution images can be taken at a working distance of $10 \mathrm{~mm}$, but to take clear images at higher resolutions, shorter working distances are required. $8 \mathrm{~mm}$ was found to be the optimal working distance for this SEM. The spot sizes on this SEM can range from 1 to 100 , where the higher the spot size, the bigger the diameter of the electron beam. Spot sizes for these images generally ranged from 5 and 30, enabling high resolution imaging of extremely small surface features.

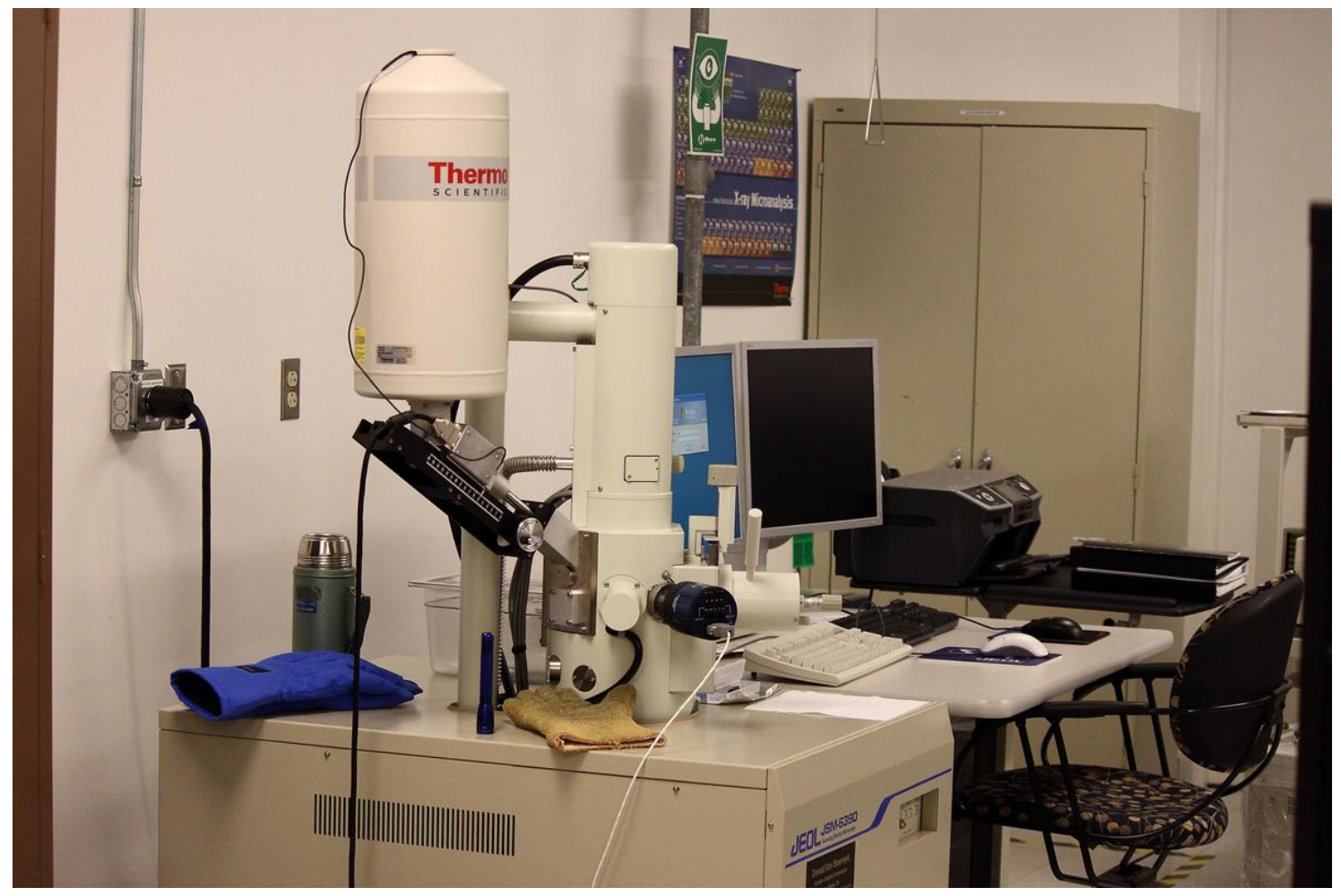

Figure 64 - The JOEL JSM-6390 scanning electron microscope was used to take topographical and crosssection images of the $\mathrm{ZnO}$ samples.

Because there were 24 different samples and both the cross-section and topography of each sample needed to be known, 48 diced samples were imaged. Cross-sectional images were obtained by placing the diced $\mathrm{ZnO}$ samples into a slotted $10 \mathrm{~mm}$ sample mount. For the cross-sectional images, the $\mathrm{ZnO}$ samples 
were scribed and diced and did not require additional sample preparation.

Topographical images were taken by dicing and placing the $\mathrm{ZnO}$ samples faceup on a $10 \mathrm{~mm}$ diameter flat sample mount with carbon tape (Figure 65). Because the $\mathrm{ZnO}$ is extremely resistive and insulating, conductive aluminum tape was attached to the samples to give the electrons a way to escape the $\mathrm{ZnO}$ and thus prevent charging. The thicknesses of the films were verified against the thickness values found through profilometry in the cross-sectional images.

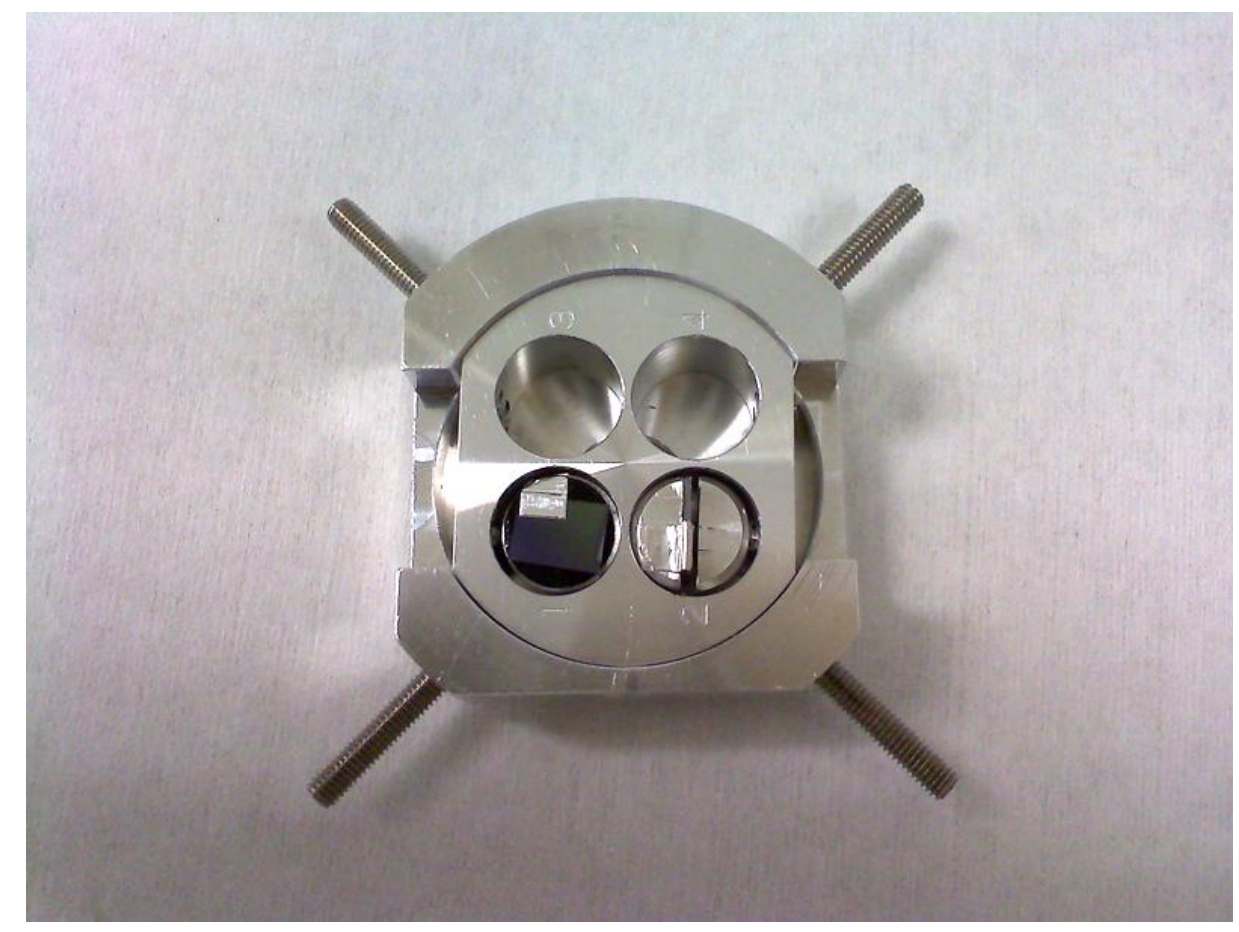

Figure 65 - To obtain cross-sectional and topographical images, ZnO samples were diced and placed into slotted and flat sample mounts and then tightened into place into a multi-sample holder with set screws.

\subsubsection{X-Ray Diffraction (XRD)}

$\mathrm{X}$-ray diffraction (XRD) was performed on as-sputtered and annealed $\mathrm{ZnO}$ samples with a Siemens D5000 Diffraktometer (Figure 66). The D5000 uses a copper x-ray source. Scans were performed between $30^{\circ}$ and $40^{\circ}$ at a scan speed of $1 \% \mathrm{~min}$ in 0.05 increments at $40 \mathrm{keV}$ and $40 \mathrm{~mA}$. 


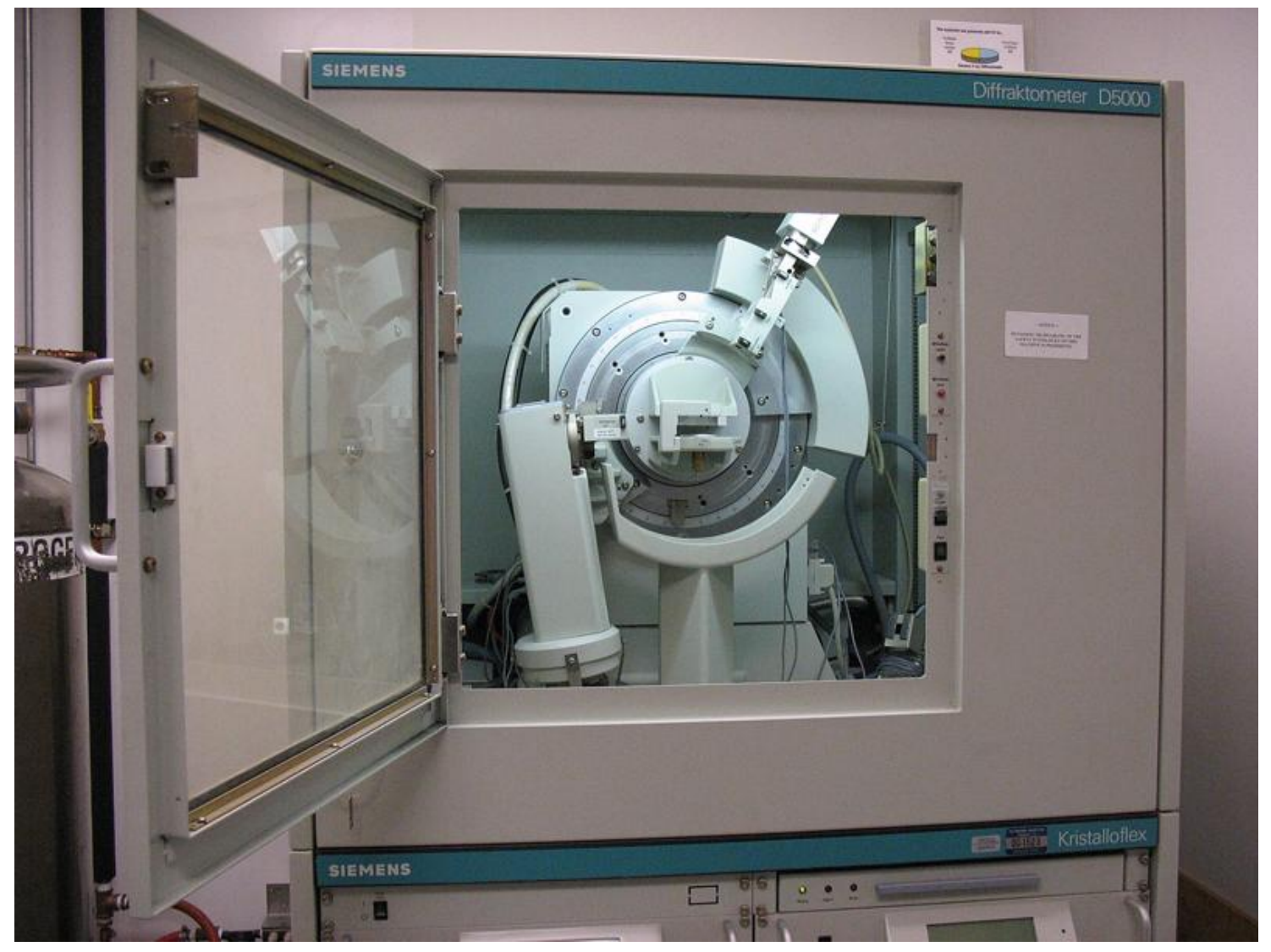

Figure 66 - In order to determine the quality of the crystals and their orientation, X-ray diffraction (XRD) was performed on the $\mathrm{ZnO}$ thin film samples.

The diced $\mathrm{ZnO}$ on silicon samples were mounted with clay into a threepronged sample holder and were leveled with a flat piece of glass (Figure 67). Before taking measurements, the XRD was warmed up on stand-by for about 20 minutes. In the XRD Commander software, the scanning parameters were entered and then the scan was started. Before opening the door to the XRD, the shutter was closed and the machine put onto standby. Once all of the diffraction peaks and their raw data were obtained using XRD Commander, they were input into the XRD analysis software, EVA, where they were analyzed. In EVA, the background noise from the scan was extracted and the patterns were smoothed with a Fourier transform function. The peaks were found and from them the $2 \theta$ 
peak angles, planar spacings $(d)$, intensity counts, and FWHM were identified. The FWHM at the peak angle corresponding to the $\mathrm{ZnO}$ crystal plane (002) were obtained using the area tool.

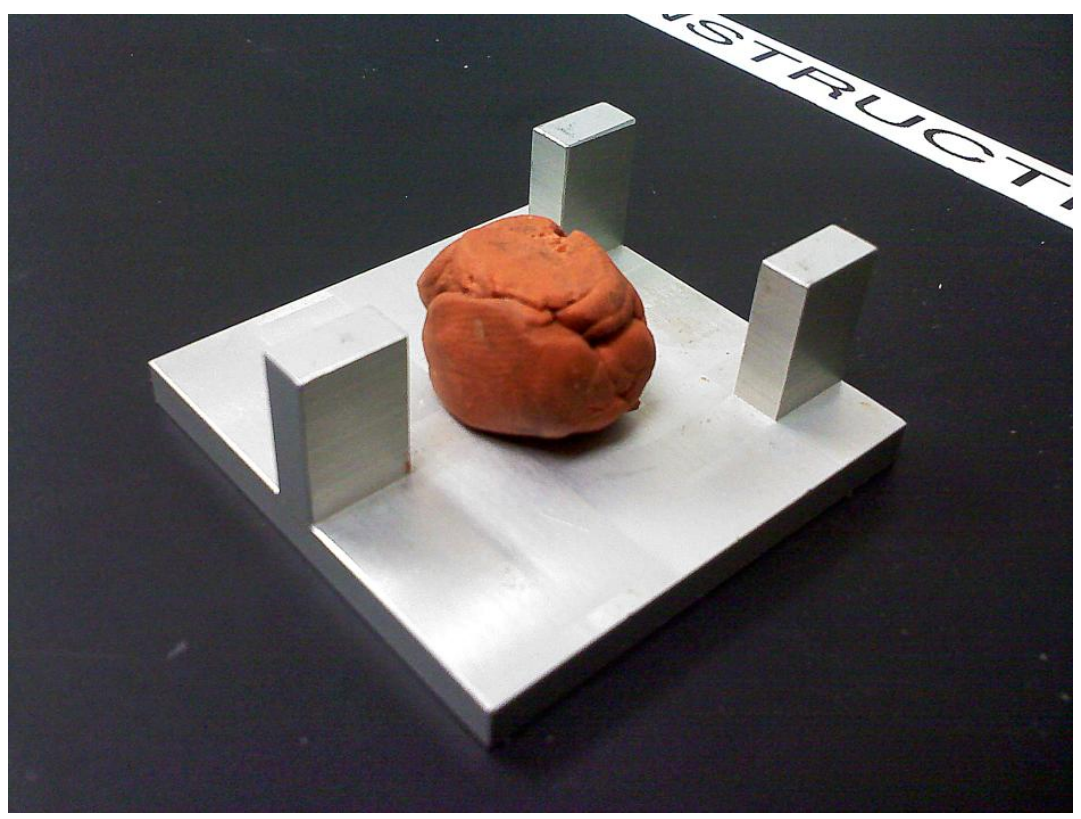

Figure 67 - The $\mathrm{ZnO}$ samples were placed onto clay and mounted onto a three-pronged sample mount which was leveled with a thick glass plate.

\subsubsection{Stylus Profilometry}

Thin film thicknesses after sputtering were determined using step heights and an Ambios XP-1 stylus profilometer (Figure 68). The stylus is housed in an acoustic isolation hood to reduce vibrational noise in the scans and placed on a large vibration damped granite block. The vertical range of the profilometer is 400 $\mu \mathrm{m}$ and its vertical resolution is $1 \AA$ at a $10 \mu \mathrm{m}, 15 \AA$ at $100 \mu \mathrm{m}$, and $62 \AA$ at 400 $\mu \mathrm{m}$. The XP-1 profilometer's stylus is diamond coated and has a tip radius of 2.0 $\pm 0.1 \mu \mathrm{m}$ with an adjustable force range between $0.05-10 \mathrm{mg}$. It also has a step-height repeatability of either $10 \AA$ or $0.1 \%$ of the nominal step height. The $\mathrm{XP}-1$ can also calculate surface roughness, waviness, and perform stress analyses on thin films. 


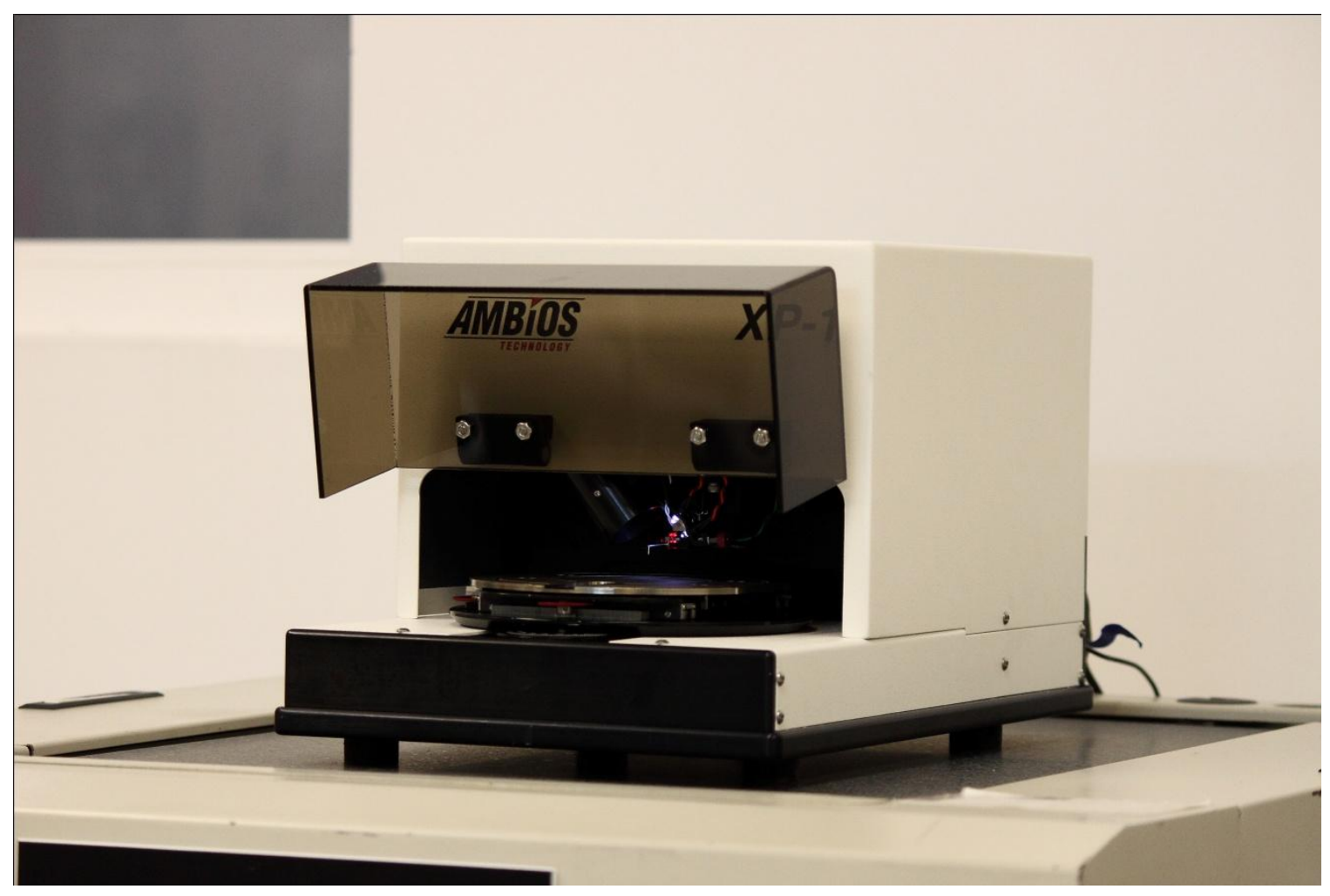

Figure 68 - An Ambios XP-1 stylus or surface profilometer was used to calculate the thicknesses of the $\mathrm{ZnO}$ thin films.

The thicknesses of the samples were determined by measuring the step height of the 24 characterization samples. The stylus would be brought close to the surface of the sample where a camera could see the interface between the $\mathrm{ZnO}$ and silicon. When the shadow of the stylus appeared in the camera, the stylus was told to engage the surface and then to perform the scan (Figure 69). 


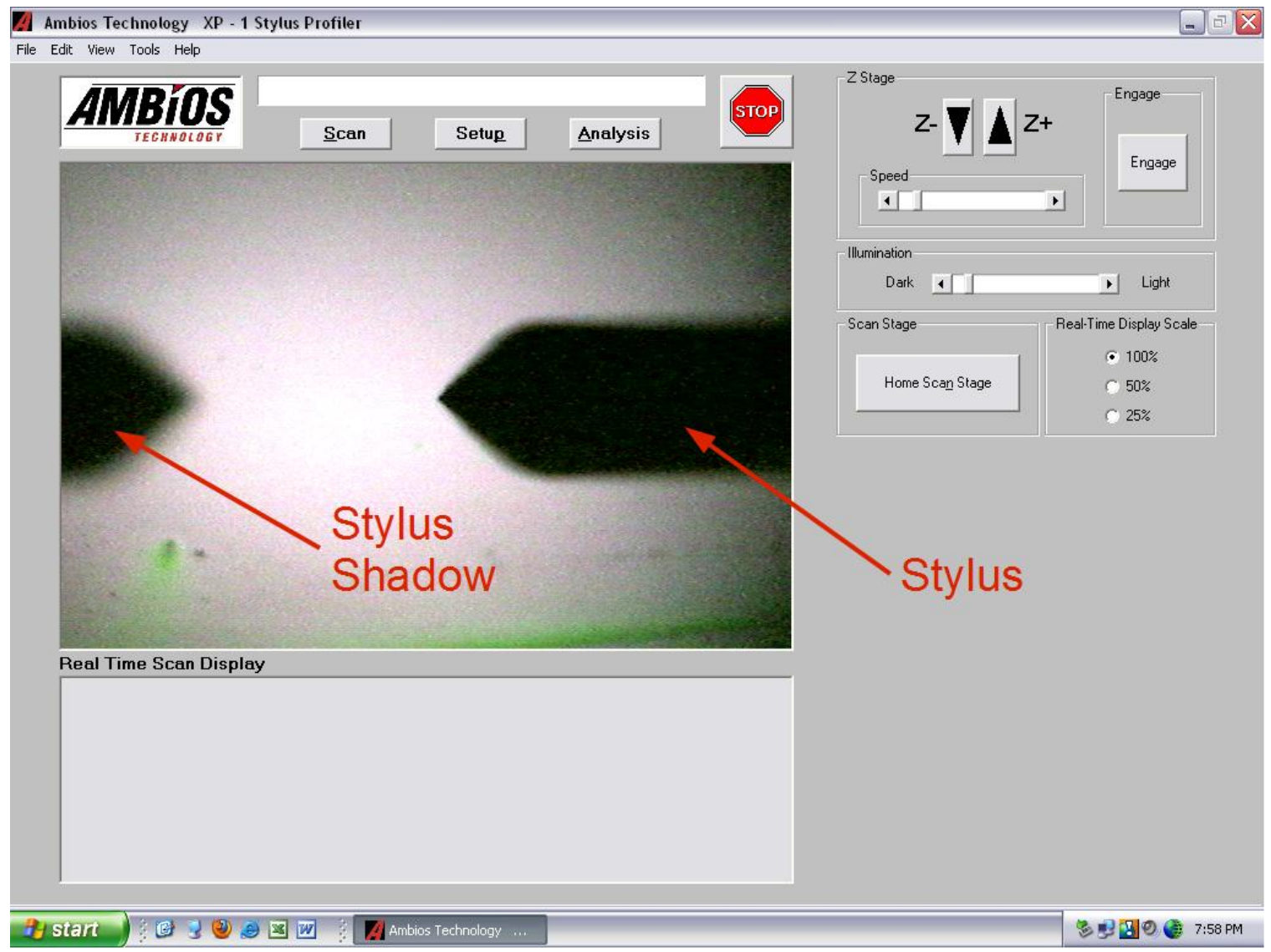

Figure 69 - As the stylus approaches the surface (on the right), a shadow of the stylus will start to appear on the left hand side of the screen. As the stylus gets closer to the sample's surface, the two will come closer together.

The step heights were obtained by placing Kapton tape on the diced samples before sputtering and then removing the tape after sputtering. Measurements were taken with a scan speed of $0.1 \mathrm{~mm} / \mathrm{sec}$, a scan length of $1 \mathrm{~mm}$, a $10 \mu \mathrm{m}$ vertical range, and $1.0 \mathrm{mg}$ of force. Three measurements were taken across the step and were averaged to get the thickness value. In addition, the two cursors in the software were resized across the film and silicon to get an even more true and averaged thickness value (Figure 70). Because the silicon surface will always be flat, the silicon was used as a reference to level the data. 


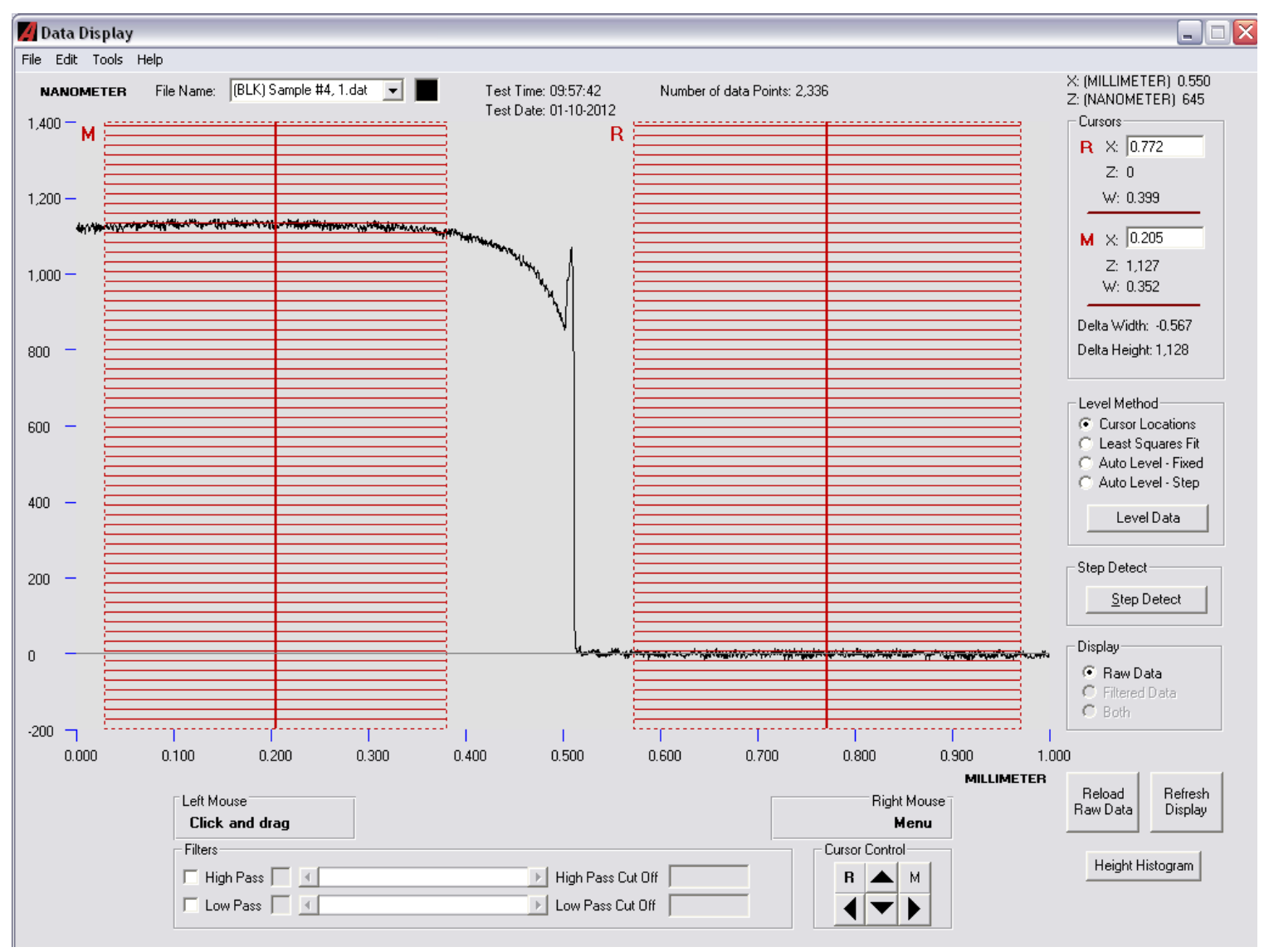

Figure 70 - To get a better representation of the change in height, the analysis cursors were resized along the surface of the $\mathrm{ZnO}$ and the silicon. The spike in the step height seen in the image above is a result of the removal of the Kapton tape creating an uneven edge on the $\mathrm{ZnO}$.

\subsubsection{Optical Properties}

The optical properties of the $\mathrm{ZnO}$ thin films were determined with a Filmetrics

F20 Reflectometer. This reflectometer features a built-in tungsten-halogen light source and spectrometer. The wavelengths the spectrometer can measure are between $380 \mathrm{~nm}$ and $1050 \mathrm{~nm}$. It measures the thicknesses of thin films using an optical technique called spectral reflectance, which emits light at the sample's surface and then analyzes the reflected light. The reflectometer can also measure the index of refraction $(n)$ and extinction coefficient $(k)$ of the material. To make these measurements, ZnO's material file containing wavelength versus refractive index values was obtained from Filmetrics and added into the 
reflectometer's material library. The Edit Structure function was used to tell the FILMeasure software that the first medium the light moved through was air, then $\mathrm{ZnO}$ of a certain thickness, and then silicon. Before taking $\mathrm{ZnO}$ measurements, a baseline measurement had to be performed using a silicon calibration wafer. To make the baseline measurement, the silicon reference/calibration wafer was measured with the spectrometer. Then a "dark" measurement was taken, where the calibration wafer was tilted $80^{\circ}$ so that the light was reflected away from the spectrometer. Afterwards, the $\mathrm{ZnO}$ samples were placed on the stage under the light from the fiber optic cable and then measured (Figure 71). Reflectance, $n$, and $k$ data at wavelengths between $380 \mathrm{~nm}$ and $1050 \mathrm{~nm}$ were recorded and then exported to be analyzed.

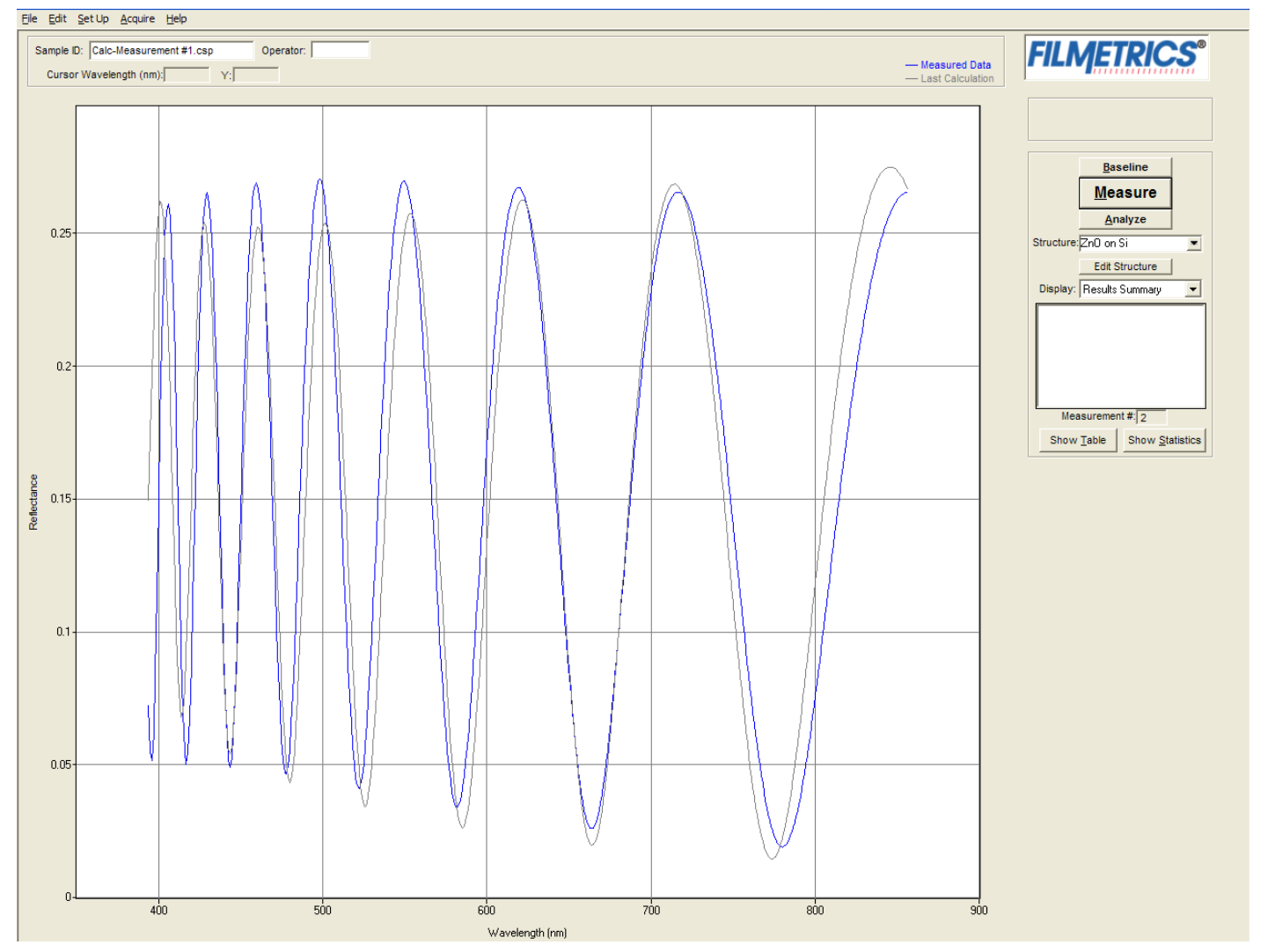

Figure 71 - Reflectance spectrums of the $\mathrm{ZnO}$ thin films were calculated and measured with a Filmetrics F20 thin-film analyzer. 


\subsubsection{Electrical Properties}

Determining the electrical resistivity and electrical conductivity of the $\mathrm{ZnO}$ thin films was attempted using a Signatone SP-2 manual four-point probe stand and a Signatone SP 4 probe head connected to a Keithley 2400 SourceMeter (Figure 72). Four-point probes feature separate pairs of current-carrying and voltagesensing electrodes, which offer increased accuracy over resistivity measuring methods with only two probes. The 4-Wire Sense mode was utilized for the measurement of sheet resistance. In this configuration, pin 1 (far left) is set to the Source HI output, pin 2 is set to Sense HI, pin 3 is set to Sense LO, and pin 4 (far right) is set to Source LO. Which pin is connected to which lead can be determined using a continuity tester on a digital multimeter. Setting up the SourceMeter in the correct configuration is essential to getting accurate measurements. 


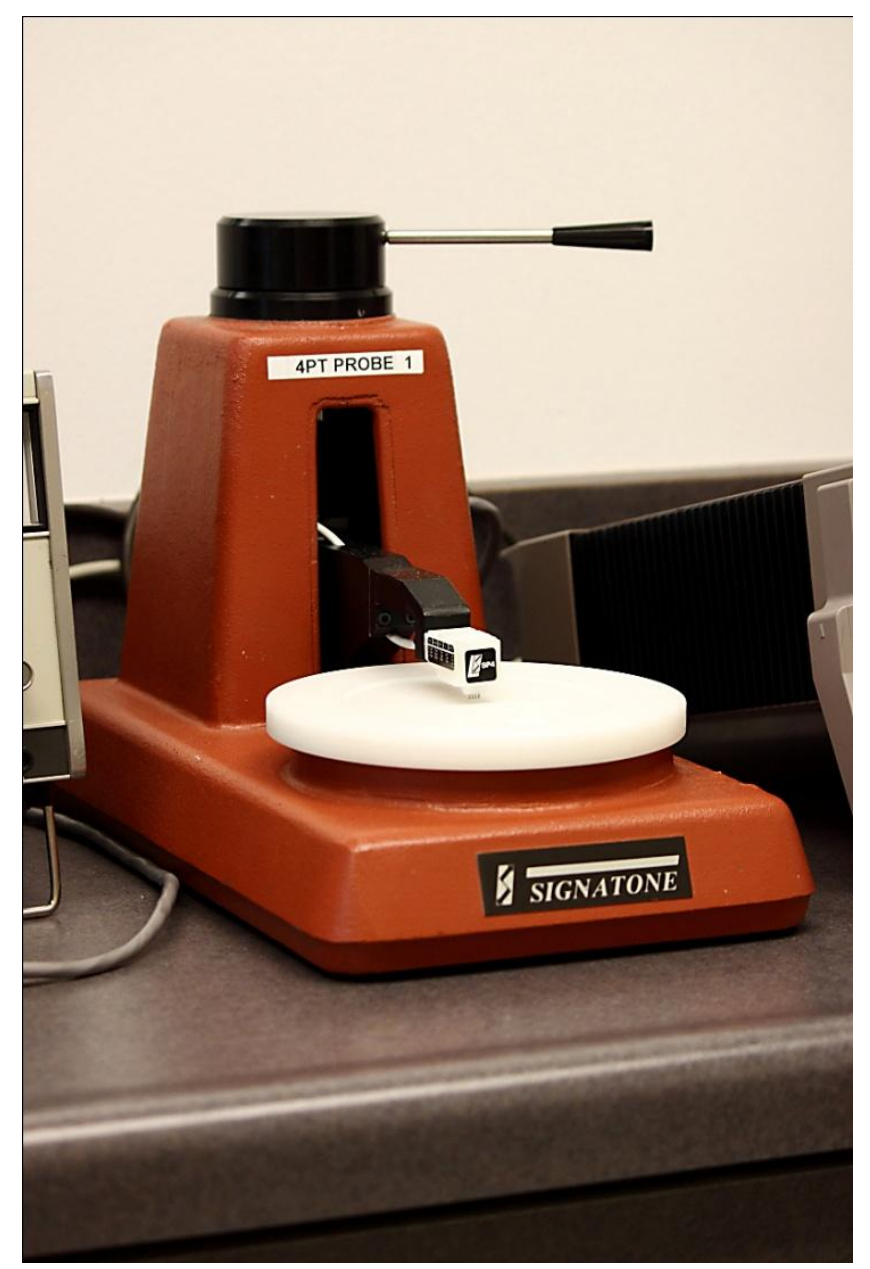

Figure 72 - The electrical resistivity of the $\mathrm{ZnO}$ films was attempted using a Signatone SP4 four-point probe head.

The SP4 heads are assembled in several different configurations of tip radius, tip material, tip spacings, and tip force. The SP4 probe head used in these measurements had tips that were made of tungsten carbide $(\mathrm{TC})$ with a tip radius of 5 mils, a tip spacing of 50 mils, 85 grams of spring pressure, and a standard flying lead termination. Direct current is supplied through the two outer pins and the voltage is measured through the two inner pins. The as-sputtered and annealed $\mathrm{ZnO}$ samples were positioned below the probe head and the probe head was lowered with the lever on the manual probe stand. Rotating the lever clockwise brings the probe head closer to the surface and rotating it counter- 
clockwise raises the head. The lever clicks when it reaches the end of its path, which is about a quarter of a turn. If the probe head does not make contact with the surface of the $\mathrm{ZnO}$ then the probe head can be lowered further with a black dial on the bottom of the probe stand. Because these $\mathrm{ZnO}$ thin films are highly resistive, you want to supply the film with the lowest amount of current possible to avoid resistive heating. The Keithley 2400 SourceMeter is capable of sourcing as little as $50 \mathrm{pA}$ of current. However, $50 \mathrm{pA}$ was too low of a current for stable measurements. 1-10 nA was a good intermediate that would sometimes give reasonable voltage measurements, but unfortunately the measurements were inconsistent and not repeatable. In order to calculate the resistivity of the $\mathrm{ZnO}$ thin films, the sheet resistance was first determined using Equation 8.

$$
R_{S}=\left(\frac{\pi}{\ln 2}\right) \frac{V}{I}
$$

Equation 8 - To calculate the resistivity of the piezoelectric material, the sheet resistance needs to be found first.

Where $R_{s}$ is the sheet resistance of the thin film, $V$ is the measured voltage from the digital multimeter, and I is the applied current from the DC power supply. Once the sheet resistance of the thin film was known, the resistivity was calculated by multiplying by the thickness of the film (Equation 9).

$$
\rho=R_{S} t
$$

Equation 9 - Once the sheet resistance of the film is known, the resistivity can be calculated.

Where $\rho$ is the resistivity of the film $[\Omega-\mathrm{cm}], R_{s}$ is the sheet resistance of the film, and $t$ is the thickness of the film [cm]. Due to the lack of repeatability, the resistivity measurements obtained for the as-sputtered samples were not used. 
However, a thick sample of $\mathrm{ZnO}$ that was sputtered at a 1:1 gas ratio at $100 \mathrm{~W}$ for 90 minutes was sent out to Signatone for resistivity measurements.

\subsubsection{Piezoelectric Properties}

The piezoelectric properties of the $\mathrm{ZnO}$ thin films were obtained using piezoresponse force microscopy (PFM). PFM is particularly effective at measuring the local piezoelectric properties of a thin film and their individual crystals, but not the bulk piezoelectric properties. These PFM measurements were performed using an Asylum Research MFP-3D atomic force microscope in the Dual AC Resonance Tracking (DART) PFM mode (Figure 73). To perform piezoresponse force microscopy on piezoelectric samples, a conductive AFM tip is necessary to make the measurements. 


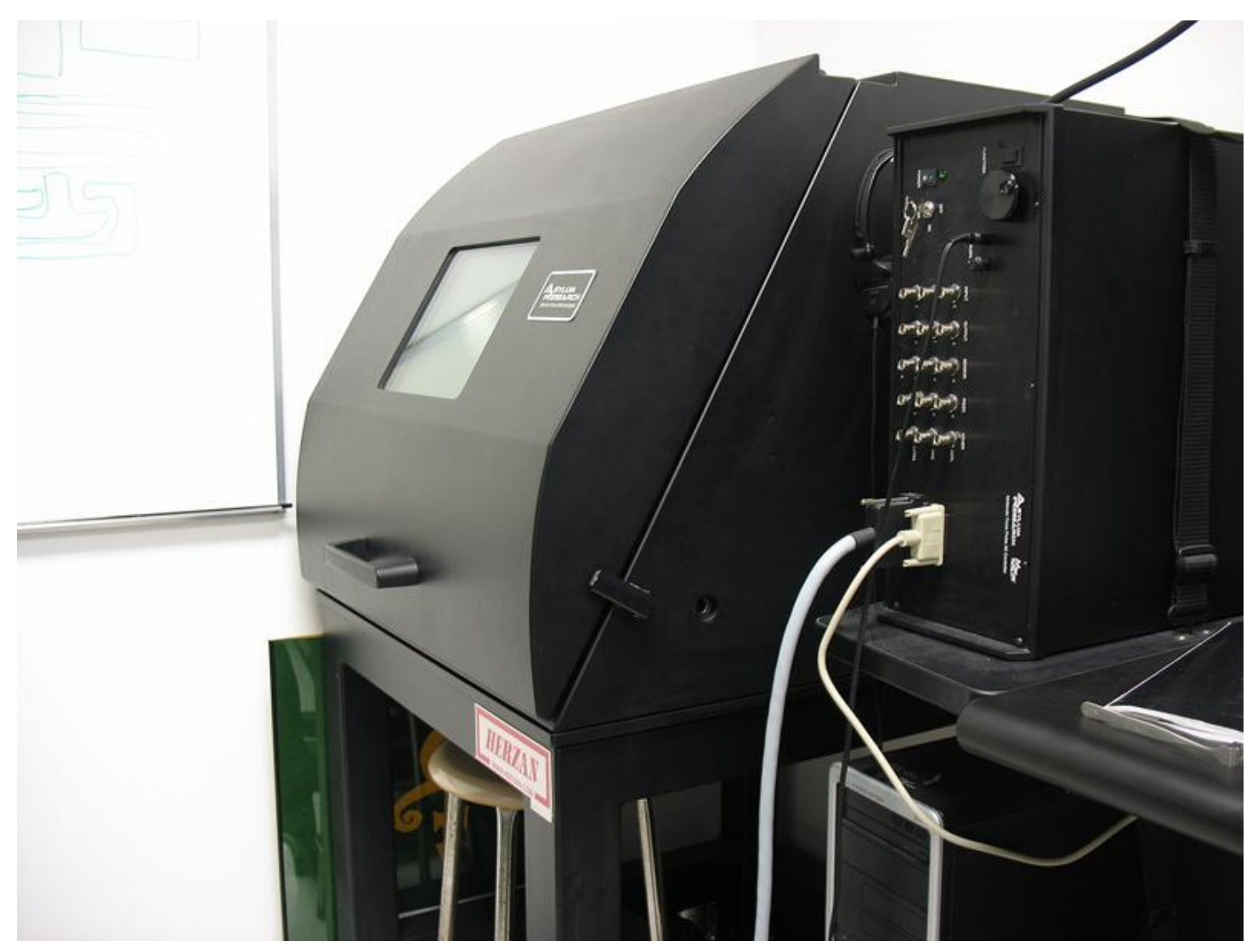

Figure 73 - The MFP-3D AFM system is enclosed in an acoustic and vibration damping housing that enables the measurement of incredible small features without the effects of noise.

The DART-PFM mode is a type of contact mode that applies two AC voltages through a conductive cantilever into the piezoelectric sample. The piezoelectric sample will deflect and change shape in response to the $A C$ voltages. The change in height can be detected by the amount of correctional voltage that must be applied to the cantilever to maintain a setpoint voltage value. The conductive cantilevers used were Olympus AC240TM silicon ElectriLevers that were purchased from Asylum Research (Figure 74). 


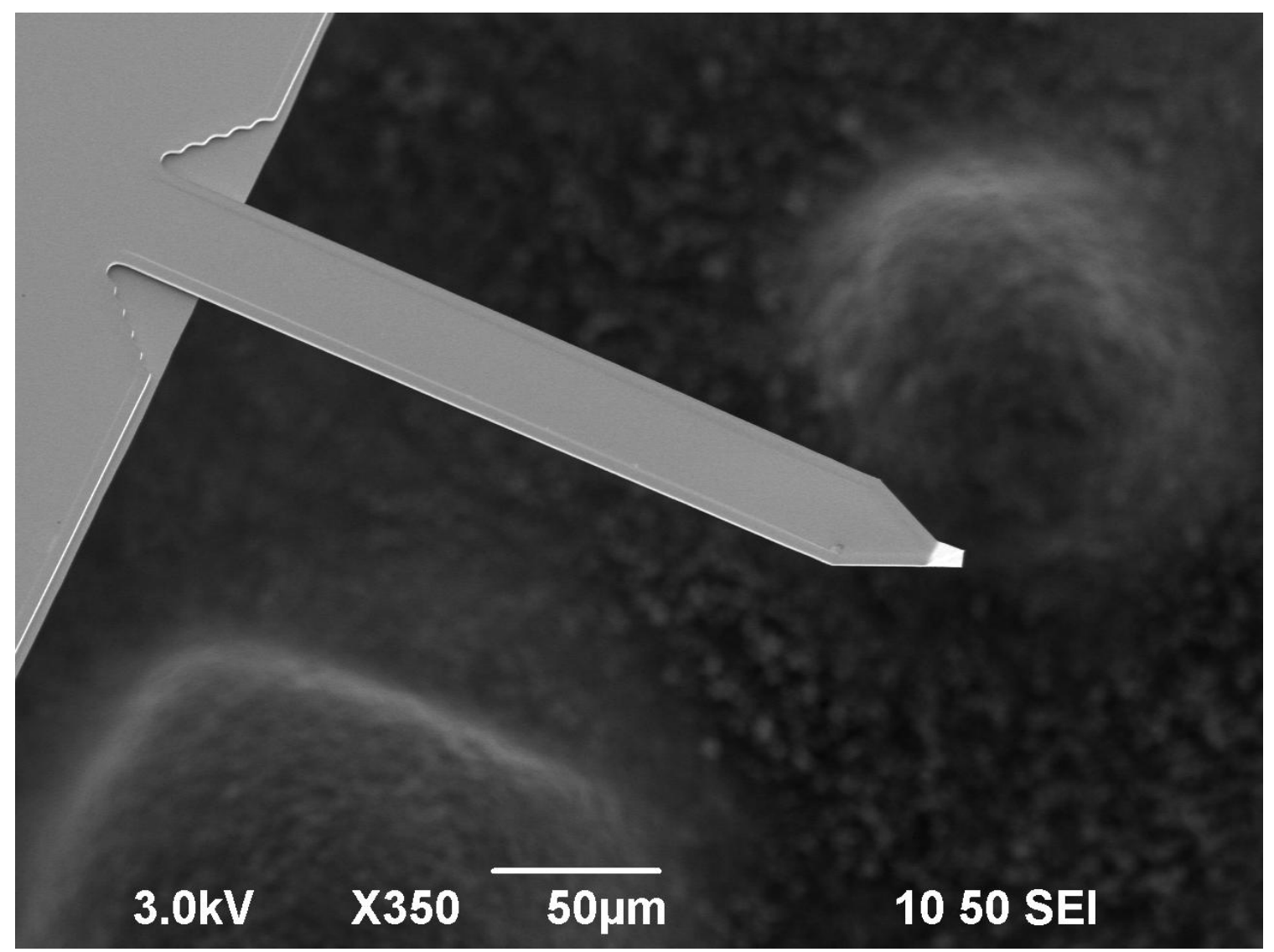

Figure 74 - The conductive cantilevers that were used in the PFM measurements were Olympus AC240TM ElectriLevers purchased from Asylum Research.

The ElectriLevers feature an aluminum reflex coating, a titanium/platinum tip coating, a $28 \pm 10 \mathrm{~nm}$ tip radius, $240 \mu \mathrm{m}$ lever length, spring constant of $2 \mathrm{~N} / \mathrm{m}$, and a resonant frequency of approximately $70 \mathrm{kHz}$. Electrilevers are the most commonly used conductive cantilevers for PFM imaging with MFP-3D machines. As a potential alternative, conductive tips were also fabricated by sputtering a thin film of gold onto the surface of VistaProbe CL-25 tips using a Denton V sputtering machine. Care was taken before and after sputtering to ensure that the tips experienced no damage. A disadvantage to using a gold coated tip is the fact that in contact mode the gold surface wears rapidly and results in an insulating tip. The way that tips can become insulating is either through the 
wearing of the conductive coating or through an insulating barrier forming on the tip and coating. Since these tips are being coated with gold, which does not form an oxide, the only way that the tip can become insulating is if the film wears off (Figure 75$).{ }^{44}$ Typically, the gold conductive film will wear off after taking an image and therefore the tips generally cannot be reused after imaging. Underneath the gold coating is a naturally thin $\mathrm{SiO}_{2}$ layer that is extremely insulating and is the reason why silicon cantilevers are not conductive. Wear rate on the coating will also depend on the hardness of the material that is being imaged, i.e. harder surfaces will wear the coating faster than soft surfaces and most imaged surfaces are harder than gold. O'Shea noted that depositing a very thick gold film and imaging in tapping mode did not reduce the rapid wear of the gold film. ${ }^{44} \mathrm{O}$ 'Shea also found that diamond coated conductive tips are the best for imaging purposes because of its hardness and chemical inertness. For these reasons, the AC240TM ElectriLevers were used for all of the PFM imaging.

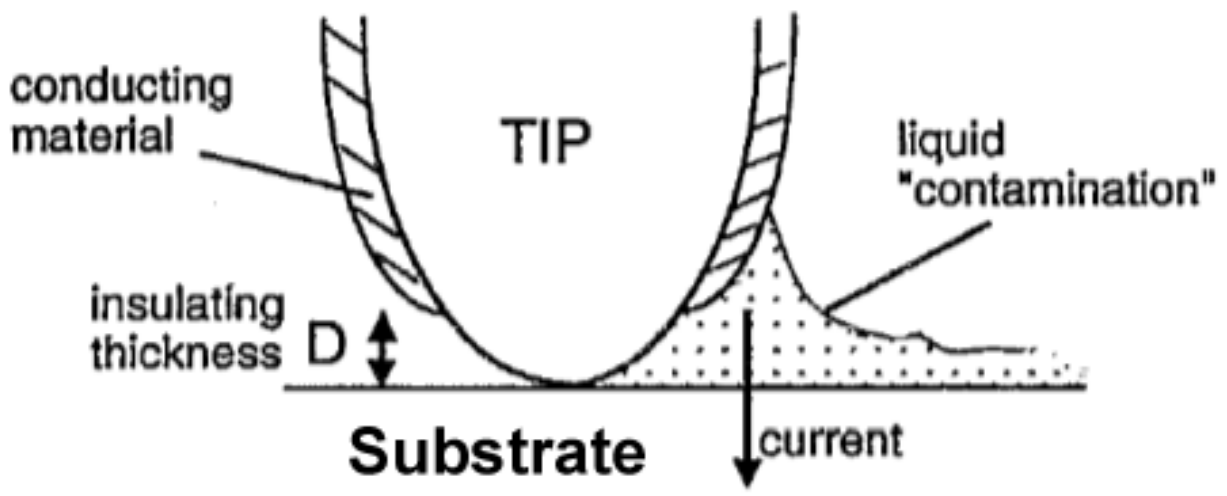

Figure 75 - The deposited conductive layer on the tip can easily be worn during imaging. ${ }^{44}$

The AC240TM cantilevers were first loaded into the MFP-3D's standard cantilever holder, while making sure that the cantilever was not crooked and not pushed too far back into the holder. Diced $\mathrm{ZnO}$ samples were taped to 
microscope slides and loaded onto the MFP-3D stage, where they were held in place by magnets. The cantilever holder was then placed into the MFP-3D's head and then the head was carefully turned over and placed onto the sample stage. Before setting all three pegs of the MFP-3D head down on the sample stage, I ensured that there was a sizable gap between the sample and cantilever tip. In the ModeMaster panel in the Igor software, the DART-PFM mode was selected, which opens up the DART Panel (Figure 76). The DART panel was used to find the contact-resonance of the tip-surface contact between the cantilever and the piezoelectric sample. It was also used to adjust the voltage applied to the conductive tip, the scan range, the Integral/DART gains, etc. The light source for the built-in optics was turned on and then the CCD camera as well. The light source and CCD camera allowed me to adjust the position of the laser onto the tip of the cantilever using the $x$ - and y-positioning knobs until the sum was maximized. 


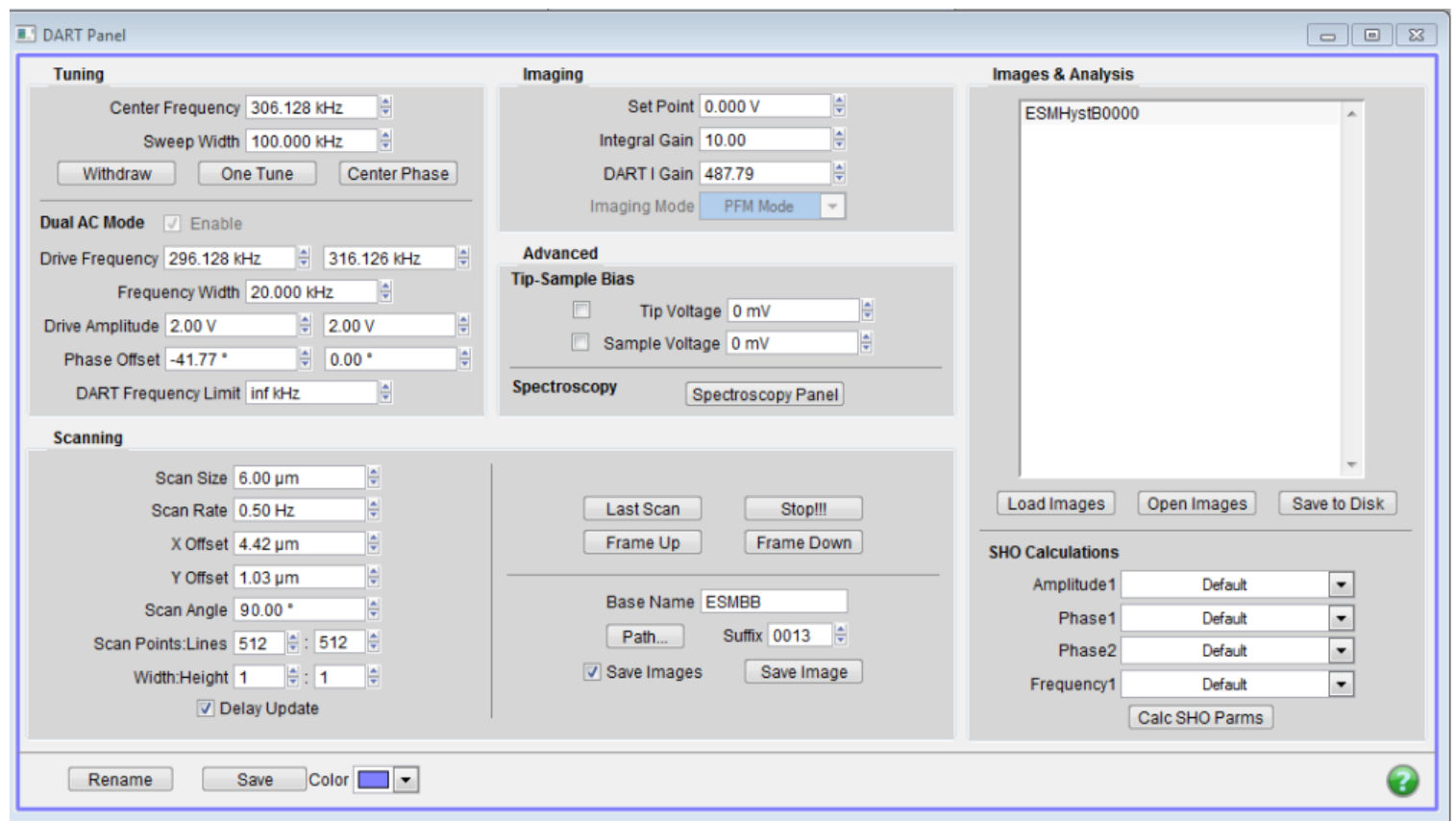

Figure 76 - The DART Panel is a unique panel for DART-PFM that allows contact-resonance tuning to be performed and the drive voltage and scanning parameters changed.

Next, the InvOLS and the spring constant of the cantilever were calibrated by opening the SpringConstTutor User Panel and following the onscreen directions. With everything calibrated, the deflection voltage was put at $-0.5 \mathrm{~V}$ with the setpoint voltage set to $0 \mathrm{~V}$ in order to achieve the $10 \mathrm{x}$ signal gain from the photodetector. The tip was lowered and engaged onto the surface and then the contact resonance was found by increasing the voltage on the tip slowly until the resulting signal voltage was above $5 \mathrm{mV}$ (Figure 77). The tip of peak should be kept inside of the red lines created by the sweep width. 


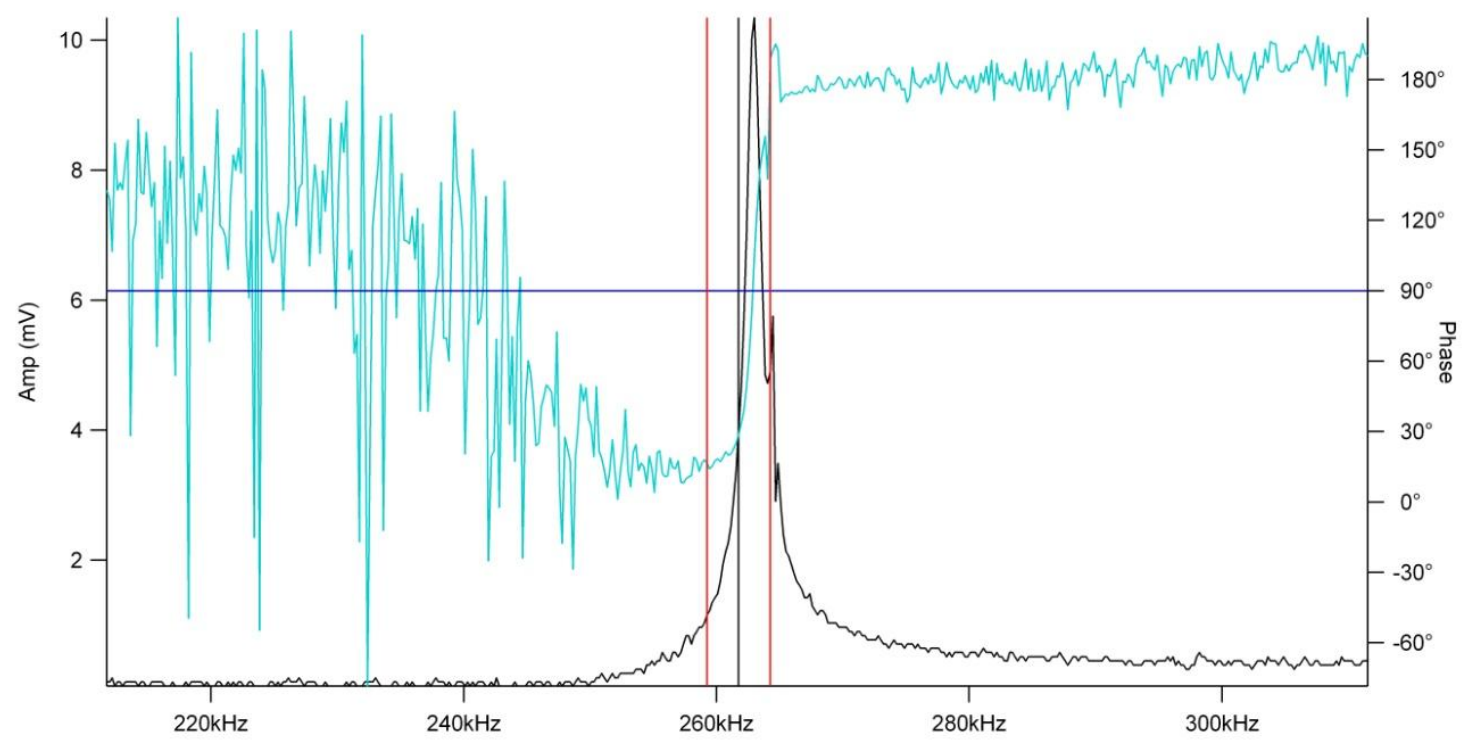

Figure 77 - The contact resonance is tuned by increasing the voltage on the tip until the received signal is over $5 \mathrm{mV}$.

The scans were started quickly after finding the contact resonance in order to avoid the contact resonance from drifting. Images with $1 \mu \mathrm{m}$ scan ranges were taken with 320 scan points and 320 lines to get a higher resolution image, but at the expensive of scan speed. The Integral Gain was typically set to around 30-40 and the DART I Gain was almost always set at 300 in order to improve the clarity of the frequency image. The data was not leveled or "fixed" in the Amplitude retrace it changed the scale and resulted in negative displacement values.

To quantify the piezoelectric response of the thin film, a few more calculations need to be performed. Once a PFM image was acquired, the image would be loaded into the DART panel and then opened. Once opened, the Calc SHO Parms button was pressed which generated four new images. SHO stands for a simple harmonic oscillator and that is how the piezoelectric and tip are modeled. The AmDR image can be used to quantify the piezoelectric response because it possesses information about the local piezoelectric coefficients of the imaged 
sample (Figure 78). The bright areas in this image represent locations that experienced the most displacement when the conductive tip was rastered over the surface. The extremely bright areas around the edges of the grains are artifacts and are not locations with high piezoelectric coefficients.

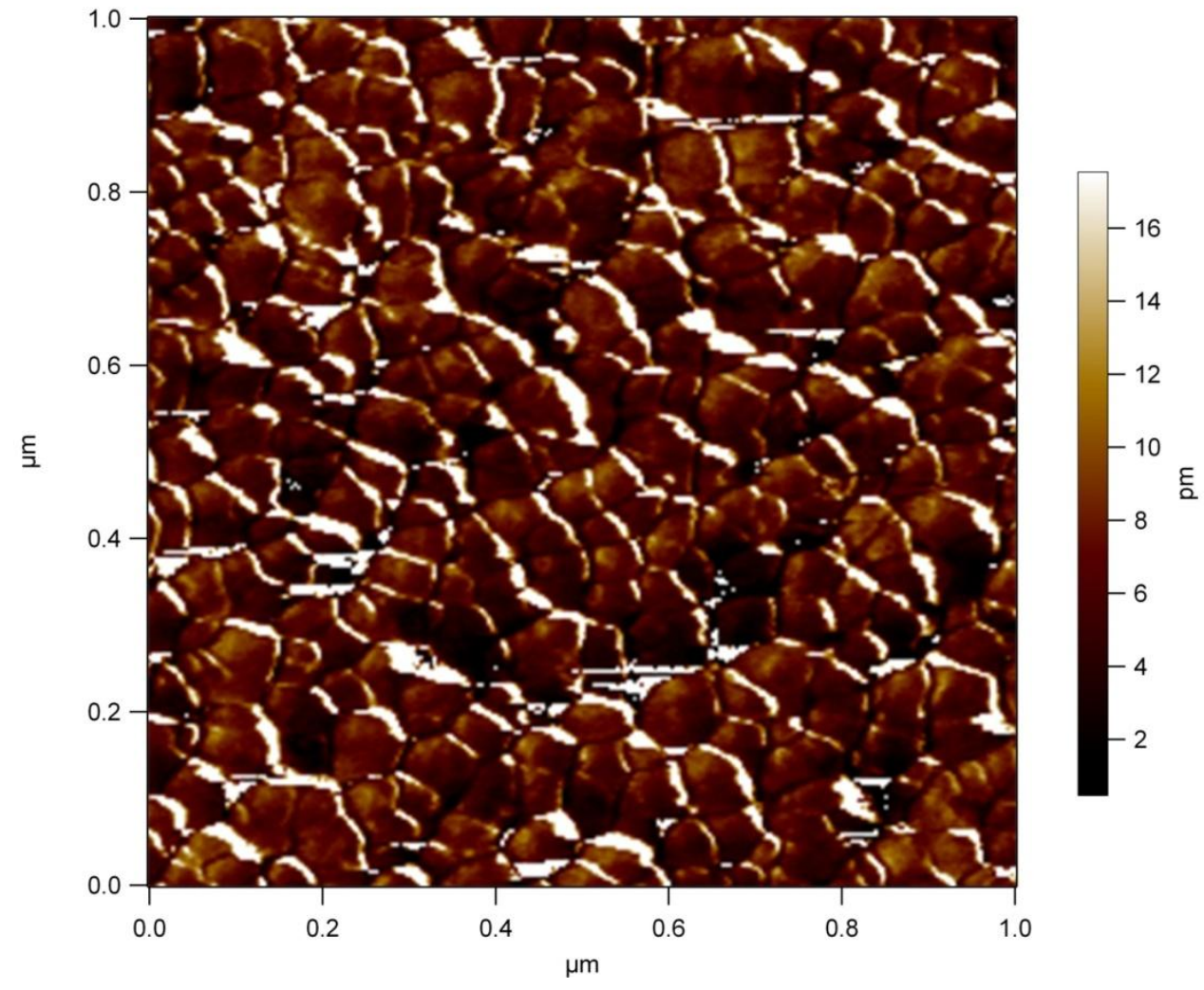

Figure 78 - The AmDR image that is generated after pressing the Calc SHO Parms button represents the local piezoelectric response of the crystals.

To get the raw data extracted from this image, a histogram needs to be created by going to the Analyze panel and selecting New Histogram (Figure 79). The software will take the raw data from the image and transport it into a convenient histogram. In the histogram panel, the data can be fitted with a few different types of distributions. I found that the Lorentzian distribution modeled the data the best. Once the data was fitted, it will gave a mean displacement 
value in units of picometers. This value is used, along with the drive voltage applied to the piezoelectric through the conductive tip, to calculate the $d_{33}$ value.

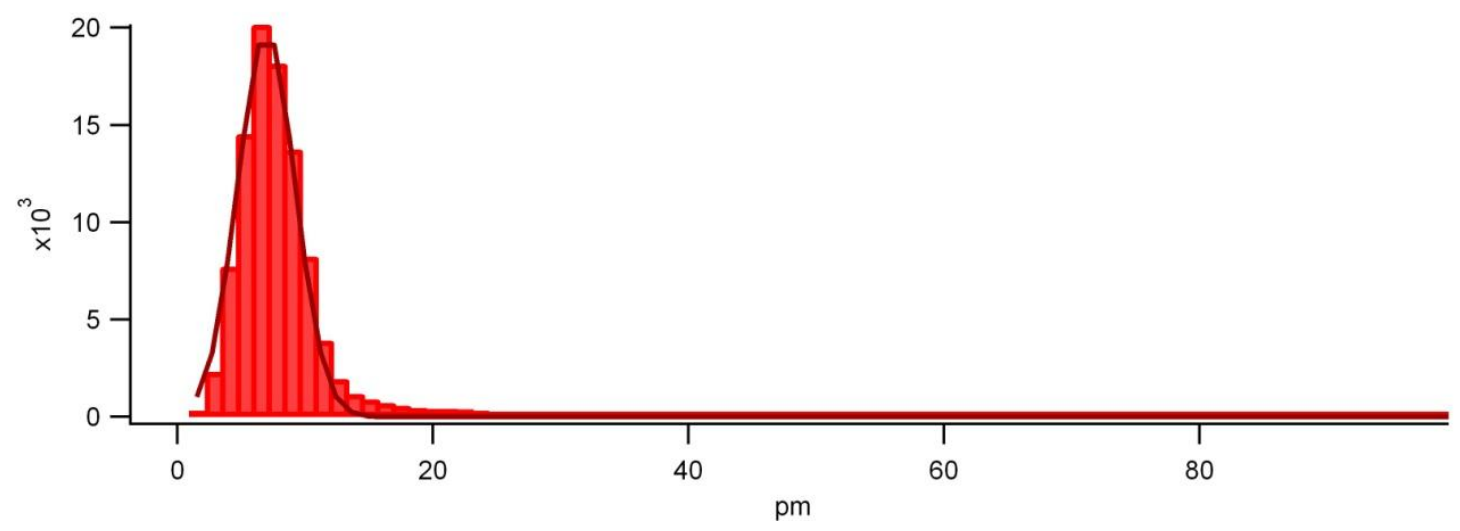

Figure 79 - The raw data is inputted into histogram form, where the mean displacement value can be determined by using a fitting the data with a Lorentzian distribution.

The $d_{33}$ value can be calculated using the simple equation:

$$
d_{33}=\frac{\Delta h}{V}
$$

Where $d_{33}$ is the piezoelectric coefficient, $\Delta h$ is the mean displacement that is obtained from the histogram, and $V$ is the voltage that is applied to the piezoelectric sample through the conductive tip. Unfortunately, this $d_{33}$ value cannot be used as a comparison to other reported $d_{33}$ values for bulk $\mathrm{ZnO}$ because this measures the local piezoelectric response of the $\mathrm{ZnO}$ and not the bulk response. However, this value can be used to as relative data to compare the piezoelectric response of the $\mathrm{ZnO}$ samples that have been sputtered. 


\section{Chapter 3: Results and Discussion}

\subsection{Microspeaker Fabrication}

Unfortunately, the $\mathrm{ZnO}$ thin film microspeakers did not function properly and did not exhibit any sound when tested with a function generator. This was due to several problems that were encountered during the fabrication process, especially during the wet etching step. The poor piezoelectric response of the $\mathrm{ZnO}$ could also be the result of a clamping effect that is caused by the adhesion of the thin film to a rigid silicon substrate. ${ }^{45}$ Because the thin film is constrained to the rigid substrate, it cannot experience any displacement or strain in the direction parallel to the substrate's surface. As a result, this can limit the displacement along the direction perpendicular to the substrate's surface and can make the piezoelectric coefficients much smaller in thin films than in the bulk.

Ko fabricated a $\mathrm{ZnO}$ piezoelectric microspeaker with a $1.5 \mu \mathrm{m}$ thick silicon nitride diaphragm and produced $76.3 \mathrm{~dB}$ SPL from a distance of $1 \mathrm{~cm}$ from the microphone with an applied voltage of $15 \mathrm{~V}_{0-p}$ at a resonant frequency of 7.6 $\mathrm{kHz} .{ }^{46} \mathrm{Ko}$ etched through all of the silicon and used a more flexible silicon nitride film as a support structure for the $\mathrm{ZnO}$ structure. Using a laser Doppler vibrometer (LDV), he found that the diaphragm was displacing $1 \mu \mathrm{m}$ at the center of the diaphragm using those frequencies and voltages. Using Equation 10 below, the pressure required to produce sound from a $4 \mathrm{~mm}$ square silicon diaphragm $10 \mu \mathrm{m}$ thick was calculated using $1 \mu \mathrm{m}$ as the amount of displacement required at the center of a square diaphragm to produce sound. ${ }^{47}$ 


$$
w_{\text {max }}=\frac{0.0138 p a^{4}}{E h^{3}}
$$

Equation 10 - The maximum deflection of the square plate was calculated at the center of the plate.

Where $w_{\max }$ is the maximum displacement at the center of the diaphragm, $p$ is the pressure applied to the silicon diaphragm, $a$ is the length of one of the sides of the square diaphragm, $E$ is the Young's Modulus for silicon, and $h$ is the thickness of the diaphragm. The pressure required to produce $1 \mu \mathrm{m}$ of displacement at the center of the diaphragm is 53.8 pascals. Despite knowing the relative piezoelectric coefficients of the $\mathrm{ZnO}$ thin films, it is difficult to predict and model the behavior of the $\mathrm{ZnO}$ film and the silicon diaphragm. I could find no simple linear equations to calculate the displacement induced by a piezoelectric film on a silicon diaphragm from applied voltages. Therefore, it is difficult to predict whether a functional $\mathrm{ZnO}$ microspeaker is possible with the current microspeaker design.

During fabrication, the initial oxide thicknesses were measured using the Filmetrics F20 thin-film analyzer (Table III). The oxide thicknesses are consistent and there is not much variance in the thickness which means that drastic heat fluctuations did not occur in the furnace. The target oxide thickness was $500 \mathrm{~nm}$ after 1 hour and 20 minutes in a $1050^{\circ} \mathrm{C}$ furnace, but the resulting oxide was about $100 \mathrm{~nm}$ thicker than was predicted by the Deal-Grove model.

Table III - Average Device Wafer Thicknesses

\begin{tabular}{|c|c|}
\hline Wafer Number & Average Thickness (nm) \\
\hline Wafer 1 & $617.3 \pm 3.3 \mathrm{~nm}$ \\
\hline Wafer 2 & $617.7 \pm 4.3 \mathrm{~nm}$ \\
\hline Wafer 3 & $616.1 \pm 4.5 \mathrm{~nm}$ \\
\hline Wafer 4 & $612.5 \pm 4.6 \mathrm{~nm}$ \\
\hline Wafer 5 & $601.9 \pm 4.9 \mathrm{~nm}$ \\
\hline
\end{tabular}


Because the wafers were left overnight and not removed after cooling to about $600^{\circ} \mathrm{C}$, this thicker than anticipated oxide could have resulted after the nitrogen was shut off when the wafers were cooling. Without the nitrogen, atmospheric oxygen in the quartz tube could have oxidized the still hot wafers and resulted in thicker oxides. Another potential and more likely possibility could be the result of the time that it took for the furnace to reach its final temperature. Even though the quartz tube was being pumped with high purity nitrogen, not all of the oxygen could be purged from the tube. Some oxidation is still bound to occur because of oxygen still present in the tube. If it took a long time for the furnace to reach its final temperature of $1050^{\circ} \mathrm{C}$, then it is possible that the wafers had more time to grow than was anticipated. However, because the thickness and quality of these oxides is not crucial for this application, an extra $100 \mathrm{~nm}$ of oxide should have little to no effect on the final device. The oxide is being used simply as a mask for the wet etching process and as an electrically insulating layer.

Since both sides of the wafer had to be processed, it was much more difficult to spin coat photoresist onto the wafer. Since there were often holes in the diaphragms after wet etching, it proved to be even more difficult to both obtain a vacuum on the vacuum chuck and to spin on an even coating. Rudimentary steps had to be taken to finish processing the wafer, for example taping aluminum foil to the back of the wafer to cover the diaphragms. Unfortunately, the wet etching process had to be the first step in the fabrication of the 
microspeakers. If the other processing steps were performed first, the structure would become damaged during the wet etching process.

\subsubsection{Wet Etching}

Due to the nature of wet etching, the fabrication of the piezoelectric microspeaker devices proved to be difficult and time consuming. Many of the variables were beyond control and were unpredictable. For example, certain areas on the wafer would preferentially etch faster or some locations would form holes. These phenomena could be the result of cold or hot spots in the TMAH etching solution because the solution is being heated by both a resistive heating element and a hot plate from the bottom. It was found that the speed of the magnetic stirring rod had an enormous impact on the surface quality of the etched surface and would also impact the etch rate. In the end, too many things had gone wrong and the final devices would not function. This was most likely due to several types of problems and not just the wet etching process. For example, at the time of fabrication the as-sputtered $\mathrm{ZnO}$ was not well characterized and not many different types of sputtering parameters were attempted. The silicon diaphragms might have been too thick as well, making them too stiff to oscillate.The fabrication of more devices from silicon was not pursued mostly because of time limitations and poor yield from the wet etching process.

Unfortunately, these silicon wafers were not ideal for the fabrication of diaphragms because they did not possess an etch-stop for the wet etching step. Because it was difficult to control the etch rate throughout the etching process, 
either a boron etch stop or silicon-on-insulator (SOI) wafer would need to be used to obtain more thickness accuracy. In SOI wafers, the selectivity of TMAH to silicon dioxide is extremely high. During wet etching I measured the oxide thickness of the wafers before and after 3 hours of etching. I found that $25 \%$ TMAH etched only $\sim 160 \AA$ in three hours, which correlates to about $53.43 \AA / \mathrm{hr}$ or $0.89 \AA / \mathrm{min}$. Compared to approximately $3800 \AA / \mathrm{min}$ of silicon, the TMAH is highly selective and allows the oxide to act as an etch stop. Similarly, silicon with boron concentrations of $2-3 \times 10^{19} \mathrm{~cm}^{-3}$ slows down the etch rate almost independently of the crystallographic orientation and can be masked with an oxide. ${ }^{43}$ However, because boron atoms are smaller than silicon atoms, using boron as an etch stop causes stresses in the resulting membrane. The boron doping also significantly increases the electrical conductivity of the silicon.

Another alternative to using SOI wafers or a boron etch stop is to use a different type of etching technique. Reactive ion etching (RIE) is often used to "dry" etch silicon using a plasma, but it is not as feasible due to the amount of time that would be required to etch through a $500 \mu \mathrm{m}$ wafer. However, it would be possible to perform both wet etching and dry etching. Wet etching would be used for the bulk of the silicon etching and then RIE could be used to obtain greater thickness accuracy and etch the rest of the way through. Alvarez fabricated thin diaphragms in silicon by performing a combination of wet etching and dry etching with success. ${ }^{48}$ This technique would be beneficial because wet etching could be stopped early when the wafers are not extremely fragile and the rest of the processing could be performed without as much risk of breaking. 


\subsubsection{Photolithography}

After becoming more familiar with the lithography and microfabrication in general, I found that patterning and masking of the electrodes may have been unnecessary. By masking and patterning, I made the fabrication of the device more complicated than it needed to be. I believe that masking could have been avoided all together and could have been solved with just Kapton tape.

After wet etching, the devices could have been fabricated in a simple five step process with little masking (Figure 80). Step 1 is to sputter a bottom electrode of aluminum. Step 2 is to place small pieces of Kapton tape above each diaphragm device. Step 3 is to sputter a thin film of $\mathrm{ZnO}$ over everything. Step 4 is to sputter the top aluminum electrode over the $\mathrm{ZnO}$. Step 5 is to remove the Kapton tape and then break off the individual devices, resulting in devices with access to the bottom electrode and top electrode. This process would coat the entire devices with aluminum electrodes and $\mathrm{ZnO}$, but that will not affect the actuation of the film. 

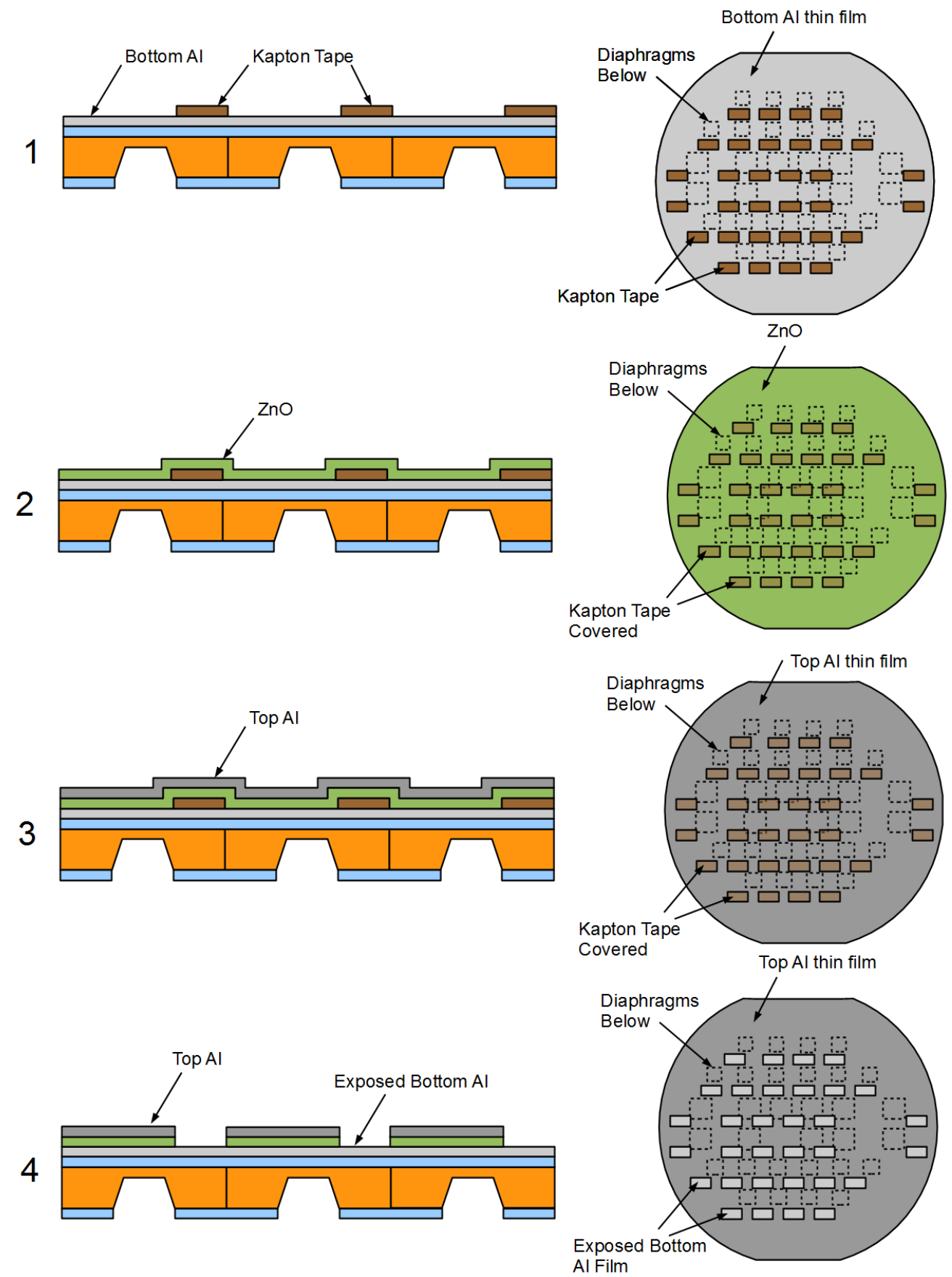

$$
\text { Al Film }
$$

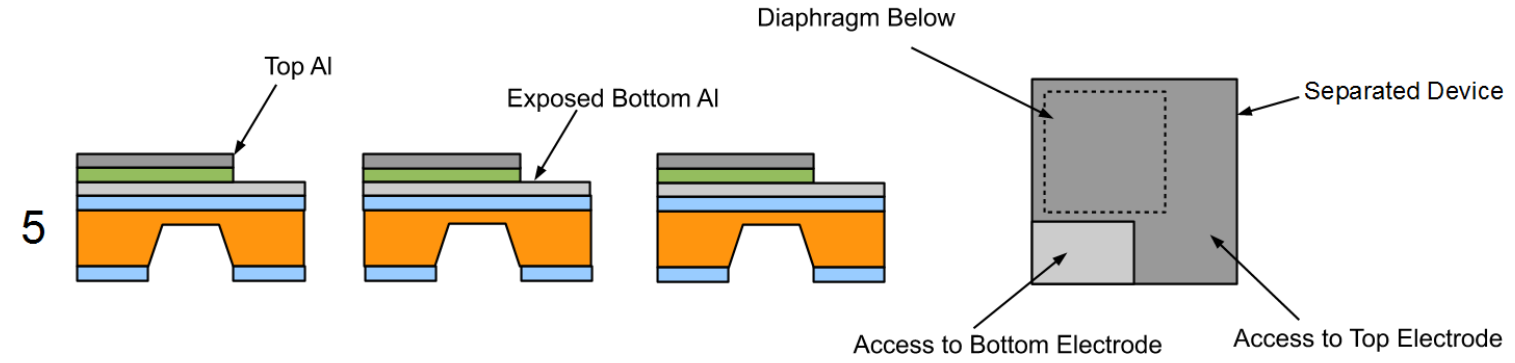

Figure 80 - Instead of a complicated photolithography process with spin coating and photoresist, a working device could be made with just Kapton tape acting as the mask. 


\subsection{Physical Vapor Deposition}

Reactive $\mathrm{DC}$ magnetron sputtering of $\mathrm{ZnO}$ is a relatively simple process, but also contains many nuances that can affect film quality and sputtering rate. Cleanliness of the chamber and its components is one of the most important parts of sputtering $\mathrm{ZnO}$. Because the $\mathrm{DC}$ sputtered $\mathrm{ZnO}$ is a highly resistive and insulating material, it can cause a phenomenon called the "disappearing" anode. ${ }^{14}$ Since the sputtered material coats nearly everything in the chamber with $\mathrm{ZnO}$, it means almost the entire chamber is partially insulating. Since the vacuum chamber and all of the parts are usually grounded and act as the anode, it may be difficult for the sputtered particles to become attracted to the substrate. Cross-contamination between different sputtering materials can also occur and affect the quality of the deposited $\mathrm{ZnO}$ films. Thoroughly cleaning the shutter, the cathode shield, the cathode holder, the stage, and the chamber every so often will prevent some of these problems from occurring and will improve the quality of the films and the plasma stability (Figure 81).

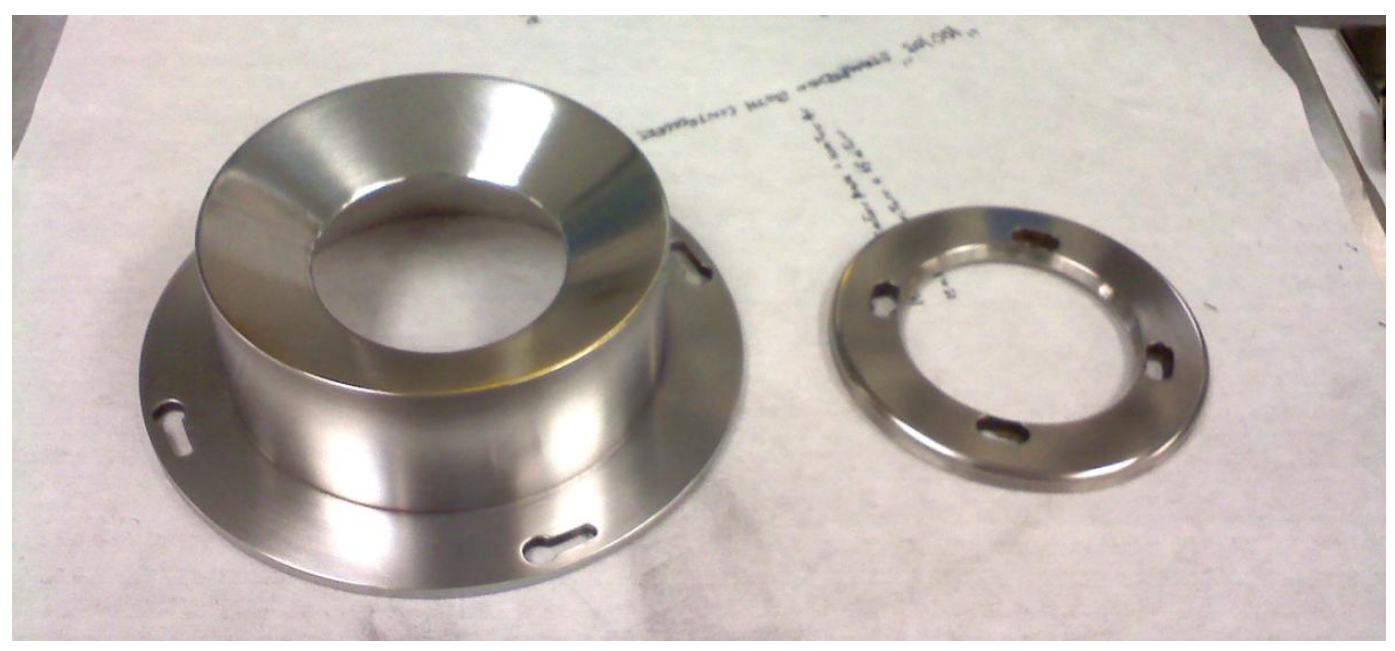

Figure 81 - Cleaning the cathode shield and cathode holder will help improve the quality of the deposited films and the plasma's stability. A clean sputtering machine is a happy sputtering machine. 


\subsection{Structural Properties}

In order for the $\mathrm{ZnO}$ crystals in a thin film to be piezoelectric, the crystals need to be oriented in the c-axis direction, which is perpendicular to the substrate's surface. If ZnO crystals are not oriented in the c-axis direction they will still be piezoelectric, but not piezoelectric in a useful direction that can be utilized in a device (Figure 82).

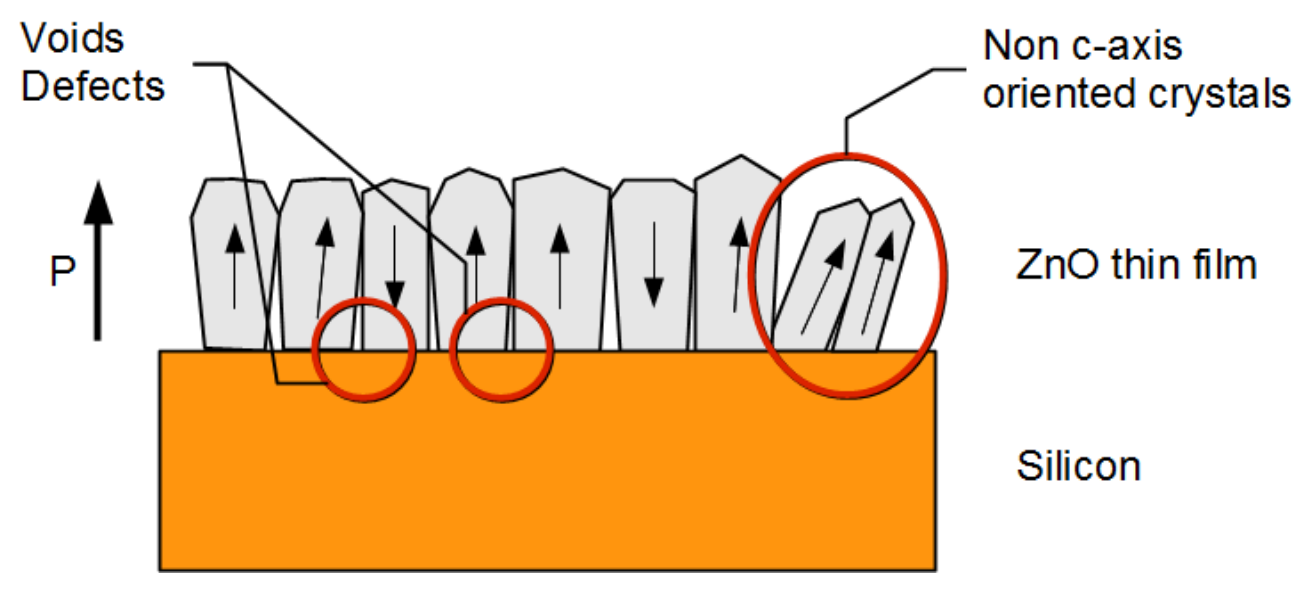

Figure $82-\mathrm{ZnO}$ crystals that are not oriented in the c-axis direction will still be piezoelectric in nature, but will be oriented in a direction that will result in smaller piezoelectric coefficients.

$\mathrm{DC}$ magnetron reactive sputtering of $\mathrm{ZnO}$ tends to form crystals that have a columnar structure in the c-axis direction. The $\mathrm{ZnO}$ crystals can also contain defects and vacancies that can affect its material properties. These structural properties were investigated with scanning electron microscopy (SEM), x-ray diffraction (XRD), and stylus profilometery. Additional XRD and thickness values can be found in the Appendix A and B.

\subsubsection{Scanning Electron Microscopy (SEM)}

In order to determine the effects that $\mathrm{Ar}: \mathrm{O}_{2}$ gas ratios, sputtering power, and sputtering time had on the structural properties of $\mathrm{ZnO}$, cross-sectional and topographical images were taken. ZnO was deposited onto 100mm n-type silicon 
wafers and imaged with a JOEL JSM-6390 scanning electron microscope. The $\mathrm{ZnO}$ samples were diced and then placed into slotted sample mounts for crosssectional imaging and flat sample mounts for topographical imaging. Through cross-sectional images, I found that most of the $\mathrm{ZnO}$ samples possessed crystals with a distinctive columnar structure that are defined by voided growth boundaries (Figure 83). These voided growth boundaries manifest because of what is called atomic shadowing, where high points on a growing surface receive more coating than the valleys, causing the high points to get higher and the valleys to get lower. ${ }^{14}$
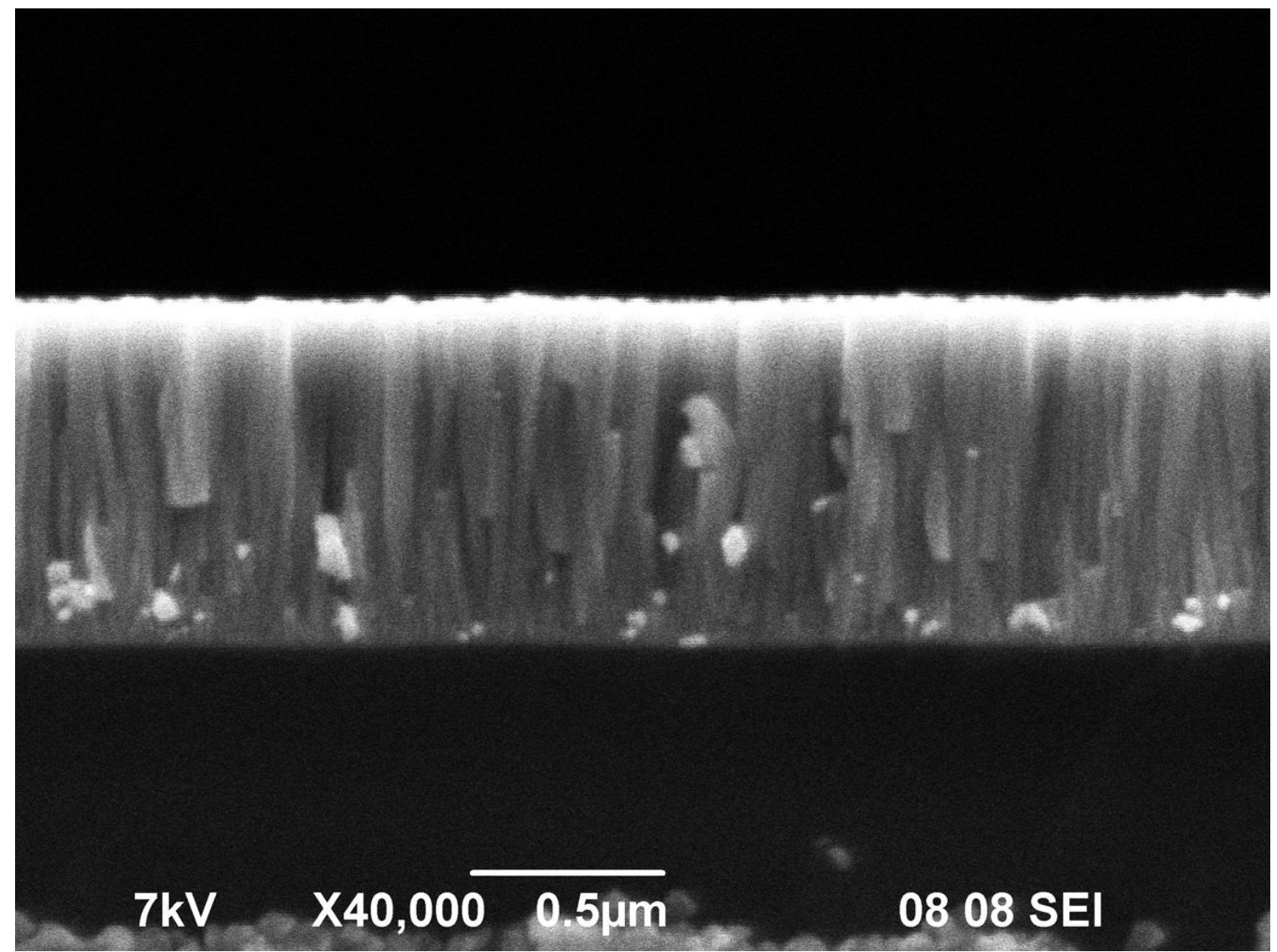

Figure 83 - The columnar structure of the ZnO crystals can be seen in this micrograph of a sample that was sputtered for 60 minutes at $100 \mathrm{~W}$ with a $1: 1 \mathrm{Ar}: \mathrm{O}_{2}$ gas ratio. 
It was difficult to determine whether some films had a columnar structure because of how thin they were. Cross-sectional images of low power and low sputtering time samples were difficult to obtain because the films could be as thin as $200 \mathrm{~nm}$ (Figure 84). These thin $\mathrm{ZnO}$ samples would reach the limits of the SEM and my skill as an operator. Images may have been difficult to obtain due to a number of issues, such as incorrect imaging parameters like accelerating voltage, spot size, and stigmation, or because of a noisy environment and the fact that the SEM is located on the second floor of a building. SEMs will operate at their best when vibrational noise is kept to a minimum.

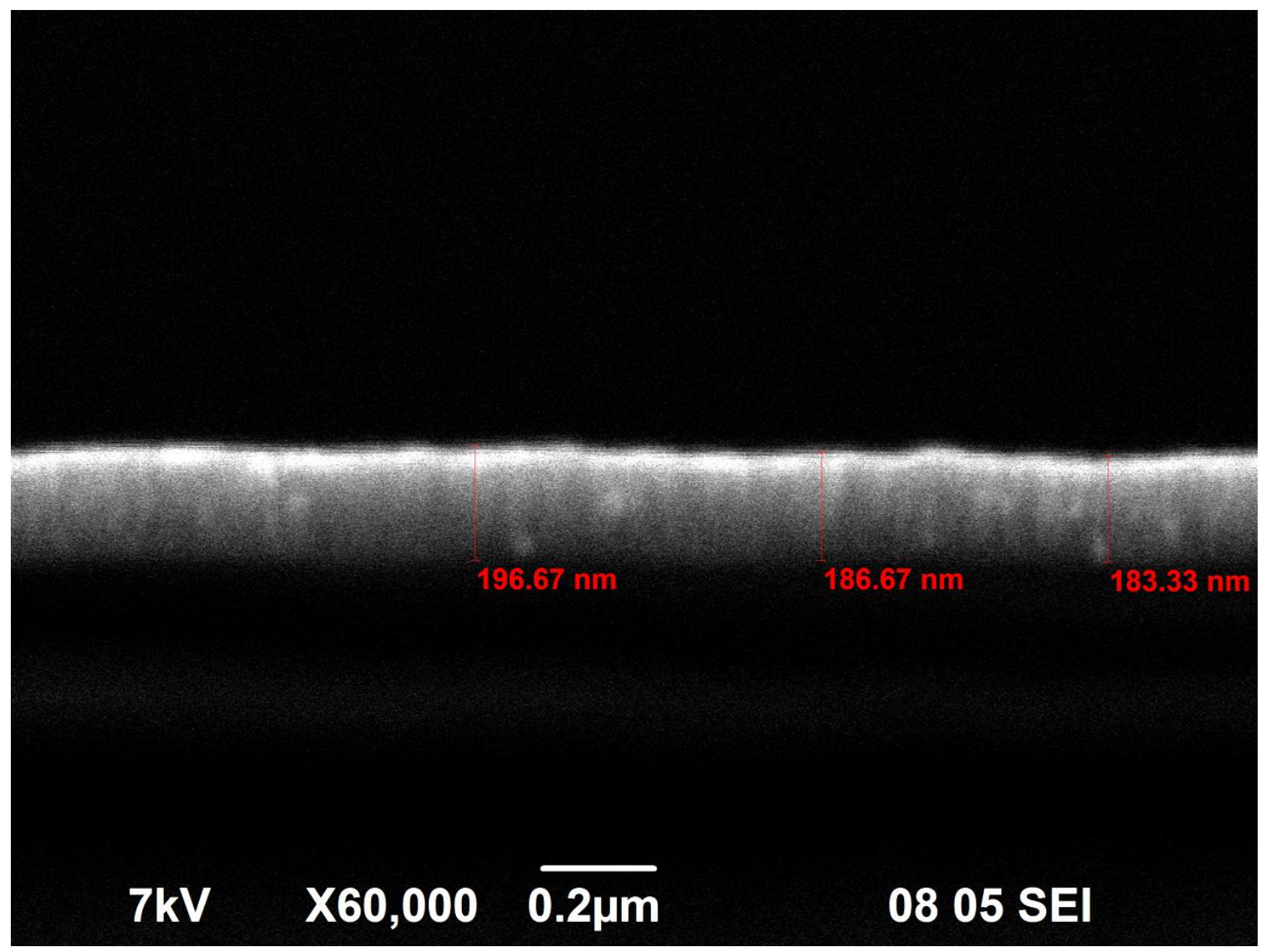

Figure 84 - This incredibly thin ZnO film that was sputtered for 30 minutes at $70 \mathrm{~W}$ with a 1:2 gas ratio was difficult to image because of how thin it was. However, a columnar structure can still be seen in the crystals. 
The effects of the argon to oxygen gas ratio on the structure of $\mathrm{ZnO}$ were investigated with cross-sectional micrographs. The four gas ratios, 2:1, 1:1, 1:2, and 1:3 were used for the comparison. The 2:1 gas ratio used $20 \mathrm{mTorr}$ of $\mathrm{Ar}$ and 10 mTorr of $\mathrm{O}_{2}$; the $1: 1$ gas ratio used 15 mTorr of $\mathrm{Ar}$ and 15 mTorr of $\mathrm{O}_{2}$; the $1: 2$ gas ratio used 10 mTorr of $\mathrm{Ar}$ and $20 \mathrm{mTorr}$ of $\mathrm{O}_{2}$; and the 1:3 gas ratio used 10 mTorr of $\mathrm{Ar}$ and 30 mTorr of $\mathrm{O}_{2}$ (Table IV)

Table IV - Gas Ratios and Partial Pressures

\begin{tabular}{|c|c|c|}
\hline Gas Ratio & Ar Partial Pressure & $\mathbf{O}_{\mathbf{2}}$ Partial Pressure \\
\hline $2: 1$ & 20 mTorr & 10 mTorr \\
\hline $1: 1$ & 15 mTorr & 15 mTorr \\
\hline $1: 2$ & 10 mTorr & 20 mTorr \\
\hline $1: 3$ & 10 mTorr & 30 mTorr \\
\hline
\end{tabular}

However, it is difficult to visually identify the differences between crosssectional pictures. For the $\mathrm{ZnO}$ samples sputtered at $100 \mathrm{~W}$ for 60 minutes at varying gas ratios, the most basic differences between the ratios are the thicknesses of the films (Figure 85). The films with greater argon pressure grew thicker than the films with greater oxygen pressures. In Figure 85a, the 2:1 gas ratio $\mathrm{ZnO}$ film was about $1.14 \mu \mathrm{m}$ thick, compared to the $885 \mathrm{~nm}$ thickness of the 1:1 gas ratio film in (b), the $775 \mathrm{~nm}$ from a $1: 2$ gas ratio in (c), and the $690 \mathrm{~nm}$ thick film from the $1: 3$ ratio in (d). There is nearly a $0.5 \mu \mathrm{m}$ difference in thickness between the 2:1 and 1:3 gas ratio samples. The difference in thickness is rather dramatic and is seen consistently in all of the films, whether they were sputtered for 60 minutes, 45 minutes, 30 minutes, or even at different powers (Figure 86, Figure 87). 


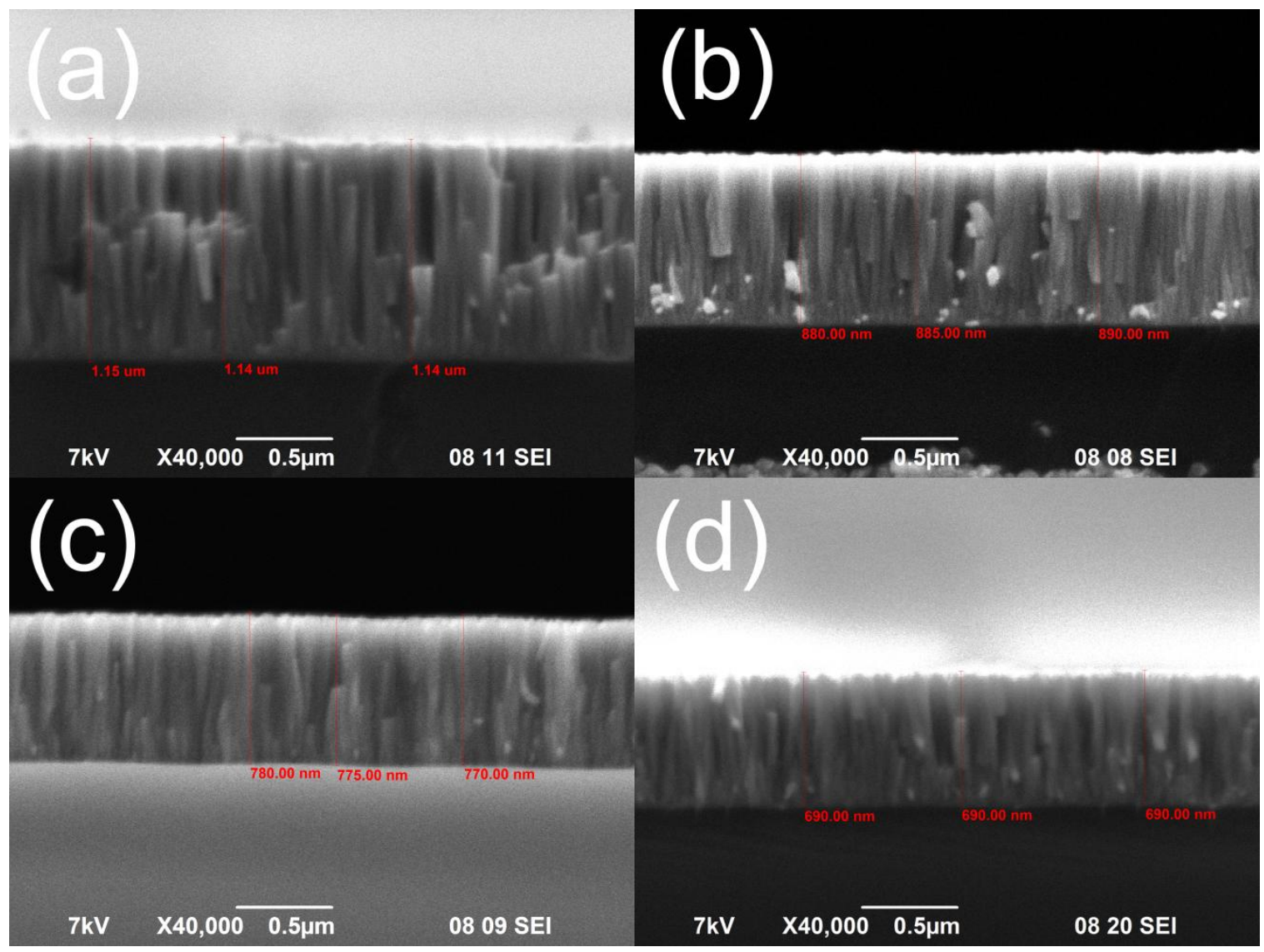

Figure 85 - The effect of gas ratios on $\mathrm{ZnO}$ that has been sputtered for 60 minutes at $100 \mathrm{~W}$ at (a) 2:1 gas ratio, (b) 1:1 gas ratio, (c) 1:2 gas ratio, and (d) 1:3 gas ratio. 


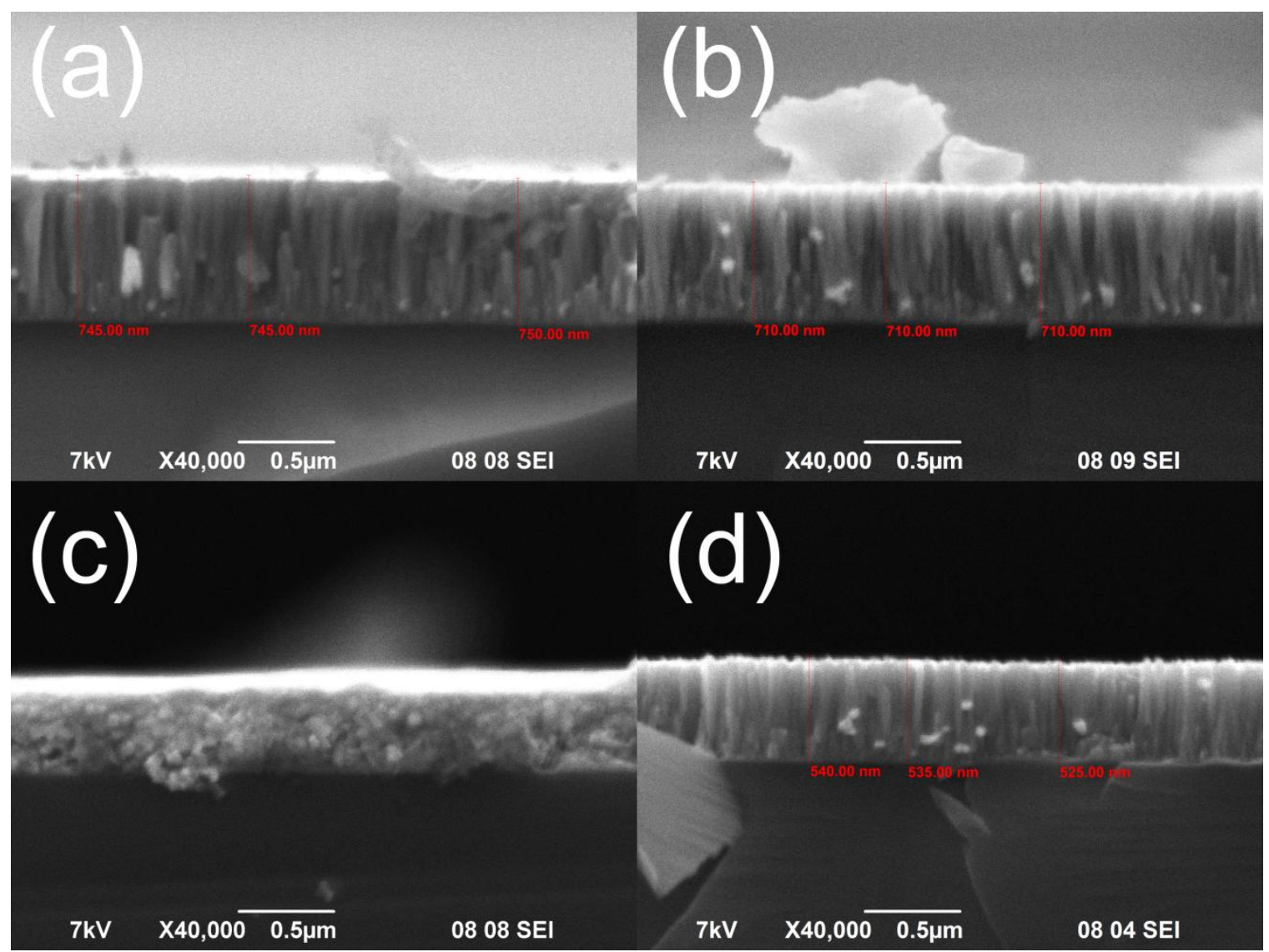

Figure 86 - Effect of gas ratios on $\mathrm{ZnO}$ samples that have been sputtered for 45 minutes at $100 \mathrm{~W}$ at a, (a) 2:1 gas ratio, (b) 1:1 gas ratio, (c) 1:2 gas ratio, and (d) 1:3 gas ratio.

The most obvious effect seen between the different sputtering times are the film thicknesses. Intuitively, the films sputtered for longer amounts of time tended to be thicker because they had more time to grow and deposit. There is also a smooth transition between the $\mathrm{ZnO}$ crystals and the substrate, which signifies good adhesion and a good quality film. ${ }^{49}$ In Figure 86c, the deposited $\mathrm{ZnO}$ with a gas ratio of 1:2 looks drastically different than the other films. In the film, the crystals do not look columnar and seemed to be scattered in all directions. This was merely the effect of a poor break when the silicon wafer was diced. Judging by the way the crystals are strewn about around the interface tells me that this is 
what caused this phenomenon. Most of the cross-sectional samples had locations similar to what is seen in this micrograph, but they were simply avoided.

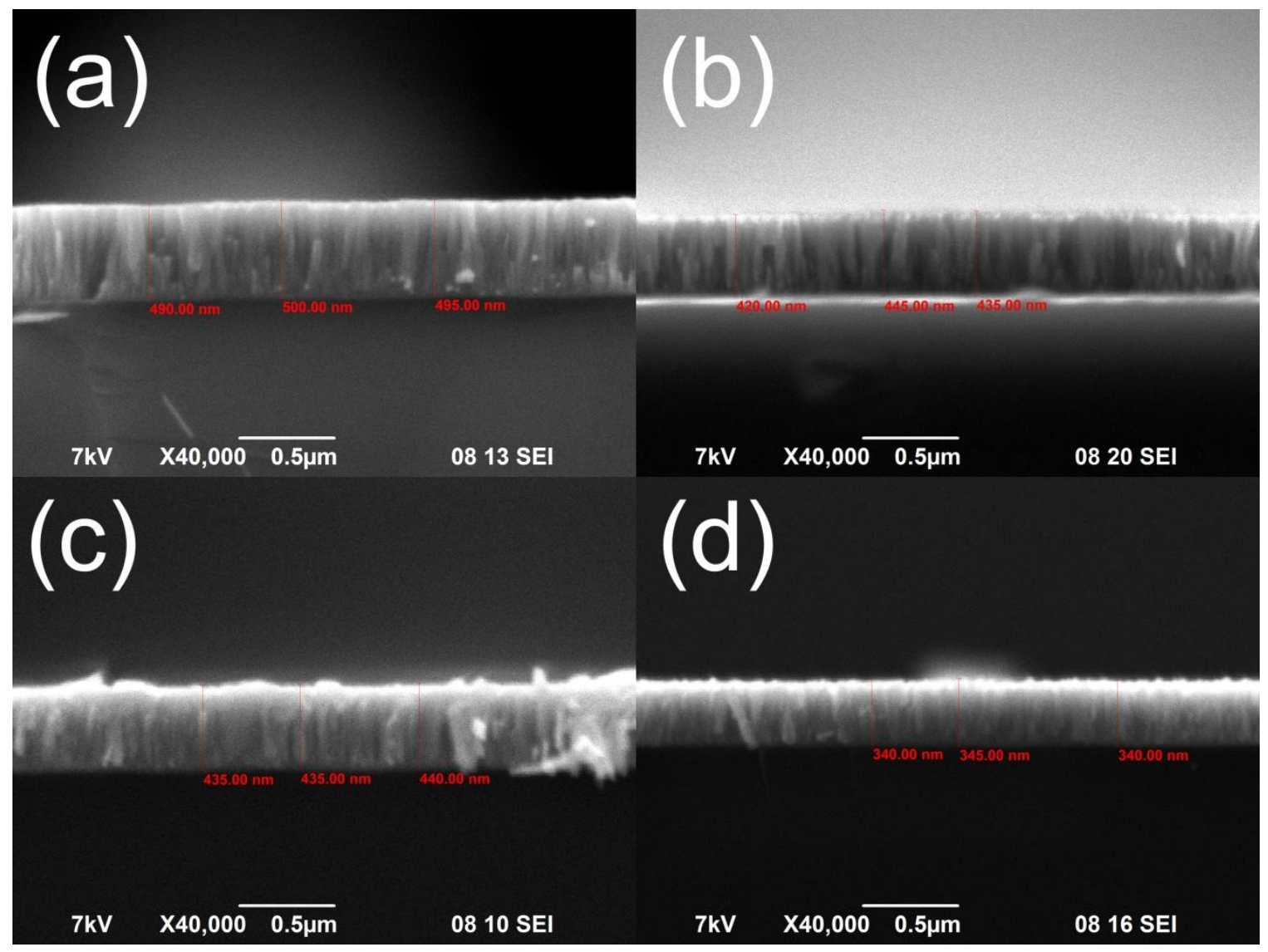

Figure 87 - The effect that gas ratios had on the structural properties of $\mathrm{ZnO}$ sputtered for 30 minutes at $100 \mathrm{~W}$ at a (a) 2:1 gas ratio, (b) 1:1 gas ratio, (c) 1:2 gas ratio, and (d) 1:3 gas ratio.

The changes in thickness with increasing amounts of oxygen content could be the result of target poisoning. A drawback to $D C$ reactive sputtering is that the chemical reactions between the $\mathrm{Zn}$ and $\mathrm{O}_{2}$ can also occur on the target's surface. This can lead to the creation of an insulating coating on the target and therefore lower current and lower sputter rates. A typical hysteresis curve of when a metal target is sputtered in a reactive gas shows that as the reactive gas flow rate increases, a sudden transition occurs where a compound forms on the target (Figure 88). ${ }^{14}$ When the compound forms, the sputter rate drops and then 
the partial pressure of the reactive gas continues to increase, which leads to further target poisoning. Another possibility is the effect of higher pressures on the mean-free path of the sputtered particles. With higher pressures in the vacuum chamber, there is more argon and oxygen particles present that can get in the way and cause collisions to occur between the sputtered particles on its journey from the target to the substrate. The total pressure for the $1: 3$ gas ratio is about $45 \mathrm{mTorr}$ (10 mTorr of $\mathrm{Ar}, 30 \mathrm{mTorr}$ of $\mathrm{O}_{2}$, and a $5 \mathrm{mTorr}$ base pressure), which is about 10 mTorr higher than the $35 \mathrm{mTorr}$ of the $2: 1,1: 1$, and 1:2 gas ratios. These higher pressures could play a role in the sputter rate and thicknesses of the films, but this is not the sole cause of the decreased sputter rates. I know this because the total pressure was constant between the $2: 1,1: 1$, and 1:2 gas ratios and yet there were still significant changes in thickness.

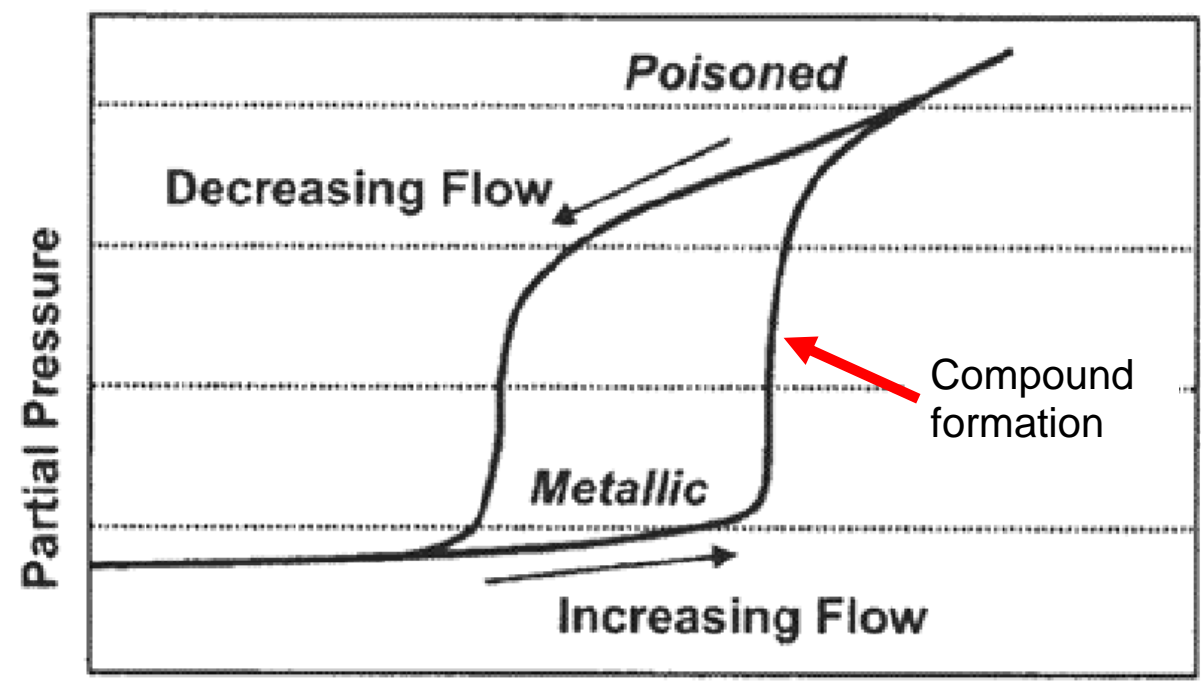

Reactive Gas Flow

Figure 88 - This typical hysteresis curve for sputtering with a metal target in a reactive gas shows how an insulating compound can form on the surface of the target and causing "poisoning" by decreasing sputter rates. 
Topographical images were also taken to see what effect the gas ratio had on the surface of the $\mathrm{ZnO}$ samples. Figure 89 shows a topographical image of $\mathrm{ZnO}$ sputtered on silicon for 60 minutes at $100 \mathrm{~W}$ and with gas ratios of $2: 1,1: 1,1: 2$, and 1:3. The most significant effect the gas ratio had on the topography was the size of the grains and the density of the grains. In the $2: 1$ gas ratio image, the crystals are densely packed and have large diameters. As the oxygen pressure increases, the grains tend to become less dense and smaller in size. The average grain size for the $2: 1$ gas ratio sample was $190 \mathrm{~nm} ; 146.33 \mathrm{~nm}$ for the 1:1 gas ratio sample; $132.67 \mathrm{~nm}$ for the $1: 2$ gas ratio sample; and $125.33 \mathrm{~nm}$ for the 1:3 gas ratio sample.

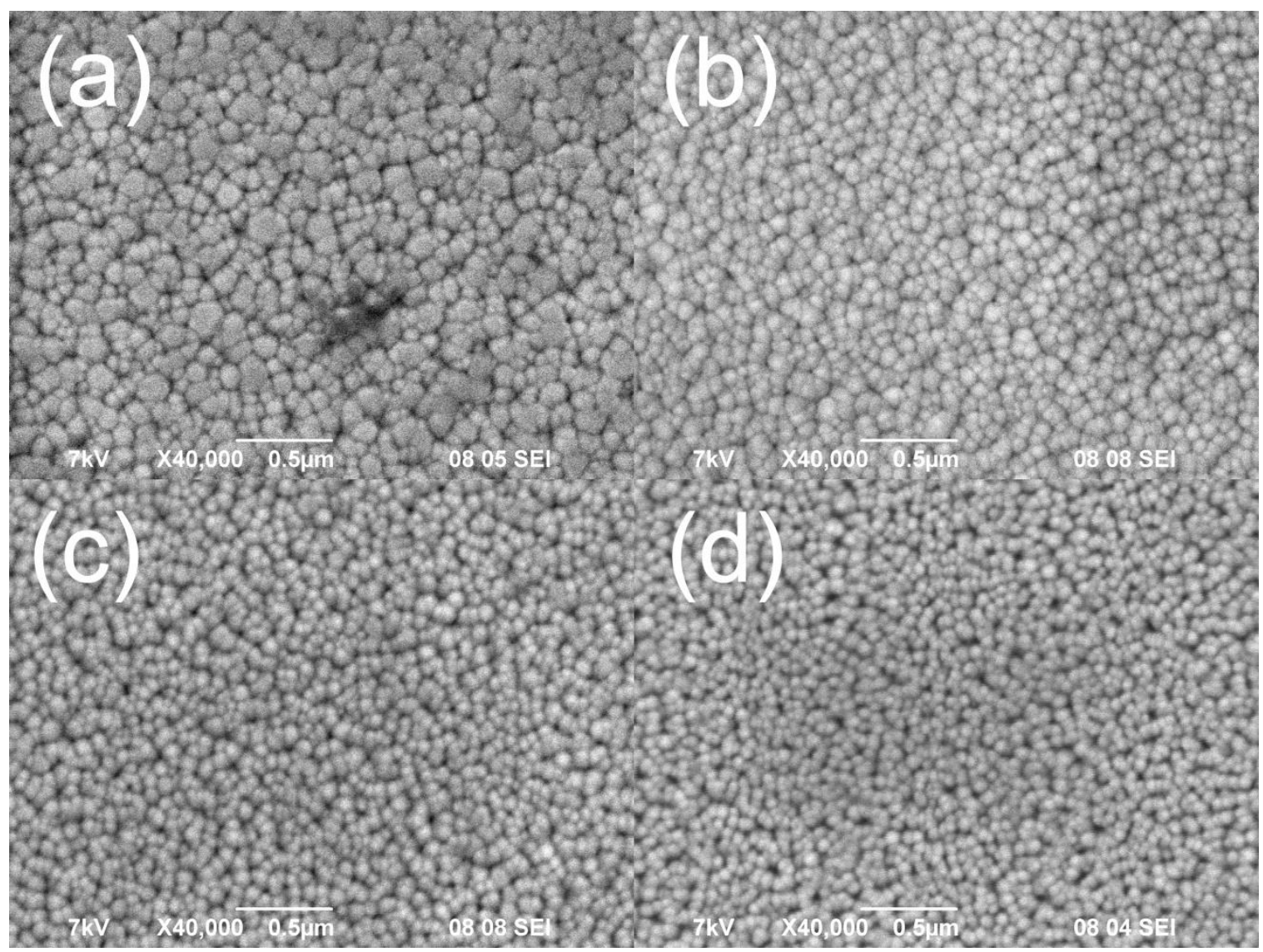

Figure 89 - Topographical image of $\mathrm{ZnO}$ sputtered for 60 minutes at $100 \mathrm{~W}$ with a gas ratio of (a) 2:1, (b) $1: 1$, (c) $1: 2$, and (d) $1: 3$. 


\subsubsection{Annealing}

To determine the effects of annealing on the $\mathrm{ZnO}$ crystals, topographical micrographs were taken of the $\mathrm{ZnO}$ surface at the same magnifications. The crystal sizes were determined using the measurement tools built into the SEM software. $\mathrm{ZnO}$ was deposited with a 1:1 argon to oxygen gas ratio. Partial pressures were 15 mTorr of argon and 15 mTorr of oxygen and the vacuum chamber reached a base pressure of $5 \mathrm{mTorr}$. $\mathrm{ZnO}$ film thicknesses were approximately $1.2 \mu \mathrm{m}$ for all the samples, which is confirmed by the cross sectional micrographs, profilometer scans, and reflectance measurements. The samples in this experiment were annealed at $600^{\circ} \mathrm{C}, 800^{\circ} \mathrm{C}$, and $1000^{\circ} \mathrm{C}$ for 30 minutes, with one sample annealed at $800^{\circ} \mathrm{C}$ for 1 hour, in a high purity nitrogen gas environment (Figure 90). My results were similar to other reported SEM images of $\mathrm{ZnO}$ post-annealed on silicon. ${ }^{50}$ The annealed samples show crystal growth as the temperature of the annealing increases. At the highest annealing temperatures, the $\mathrm{ZnO}$ crystals started to experience crystal modification in the form of melting. Under XRD measurements, these annealed samples have increased crystal alignment, which is shown by a more narrow or decreasing FWHM value. There is also a slightly increasing peak angle for the (002) crystal plane due to the relaxation of the films. ${ }^{50}$ 


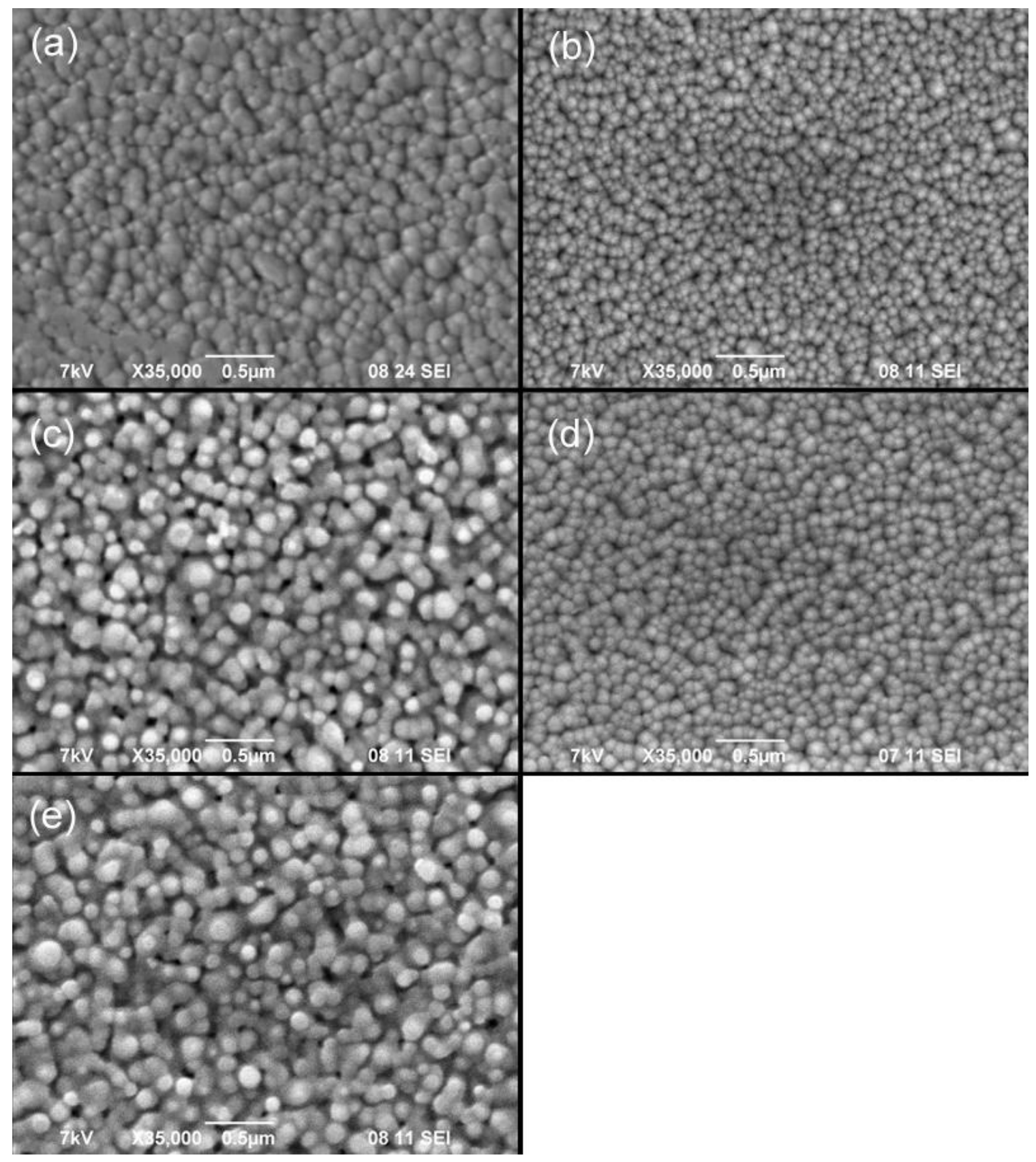

Figure 90 - SEM micrographs of 1:1 $\mathrm{Ar}: \mathrm{O}_{2}$ deposited $\mathrm{ZnO}$ annealed for 30 minutes at varying temperatures. (a) As sputtered $\mathrm{ZnO}$ control sample with no annealing, (b) $\mathrm{ZnO}$ annealed at $600^{\circ} \mathrm{C}$, (c) $\mathrm{ZnO}$ annealed at $800^{\circ} \mathrm{C}$ for 30 minutes, (d) $\mathrm{ZnO}$ annealed at $800^{\circ} \mathrm{C}$ for 1 hour, (e) $\mathrm{ZnO}$ annealed at $1000^{\circ} \mathrm{C}$.

Cross-sectional images of the annealed samples were also taken and with them the structural changes are extremely apparent (Figure 91). Instead of the edges of the $\mathrm{ZnO}$ crystals being sharp and well defined with distinct voided growth boundaries like in Figure 91a, the boundaries have started to meld 
together. This post-annealing process has caused the $\mathrm{ZnO}$ crystals to undergo almost a kind of sintering process, where the atoms in the crystals start to diffuse across the boundaries to the other crystals. Schuler encountered the same phenomenon when annealing $\mathrm{ZnO}$ samples deposited on silicon. ${ }^{14}$

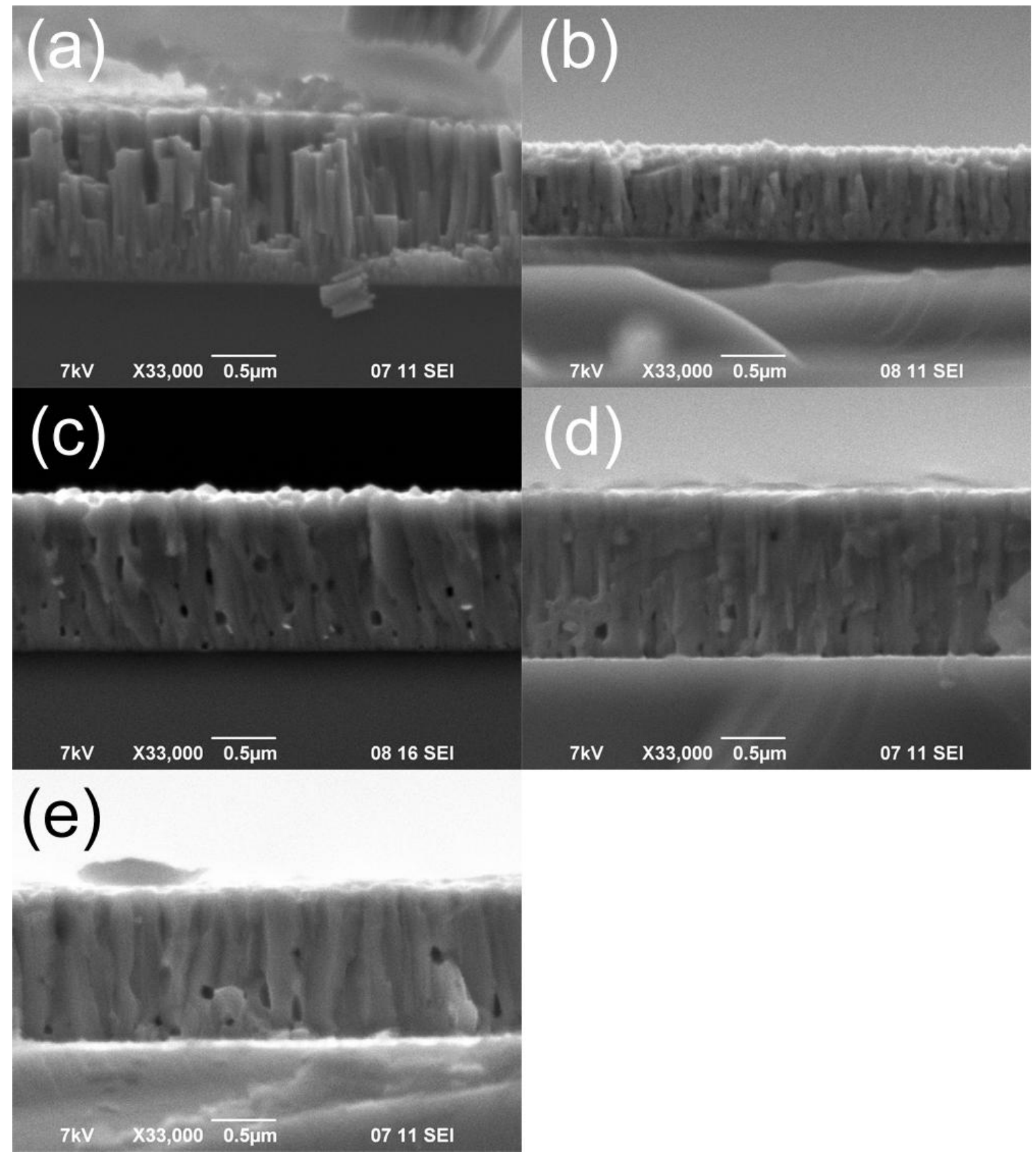

Figure 91 - ZnO thin films deposited with a 1:1 gas ratio were annealed in a high-purity nitrogen environment at various temperatures and times: (a) as-sputtered, (b) $600^{\circ} \mathrm{C}$ anneal for 30 minutes, (c) $800^{\circ} \mathrm{C}$ anneal for 30 minutes, (d) $800^{\circ} \mathrm{C}$ anneal for 1 hour, (e) $1000^{\circ} \mathrm{C}$ anneal for 30 minutes. 
This closing of the large, voided boundaries between the crystals would explain the increased electrical conductivity of the annealed samples. Without the voided boundaries, the electrons can travel across and through the crystals unhindered by large the gaps that were previously between the crystals. DC sputtered $\mathrm{ZnO}$ tends to form larger sized grains, which promotes the formation of these highly resistive grain boundaries that inhibit the transfer of charge between individual crystals. This crystal separation of DC sputtered $\mathrm{ZnO}$ also enhances the film's piezoelectric properties. ${ }^{14}$ The annealing process improved the piezoelectric response of the $\mathrm{ZnO}$ at $600^{\circ} \mathrm{C}$, but the piezoelectric response decreased at higher annealing temperatures.

\subsubsection{X-Ray Diffraction (XRD)}

Diffraction patterns for the sputtered $\mathrm{ZnO}$ samples were obtained through $\mathrm{x}$ ray diffraction $(\mathrm{XRD})$. The $\mathrm{ZnO}$ samples were sputtered onto diced $\mathrm{n}$-type silicon wafers with different combinations of gas ratios, sputtering powers, and sputtering times. The presence of a diffraction peak around $34^{\circ}$ corresponds to the c-axis oriented $\mathrm{ZnO}$ crystal growth along the (002) crystal plane. This c-axis crystal orientation is essential to providing useful piezoelectric properties for devices. The full-width at half-maximum (FWHM) values, which indicate crystalline quality, were also obtained from the XRD measurements. Slight shifts in the (002) peak position indicate relaxation of the stresses in the film, which is especially apparent in the annealed samples (Figure 92). 


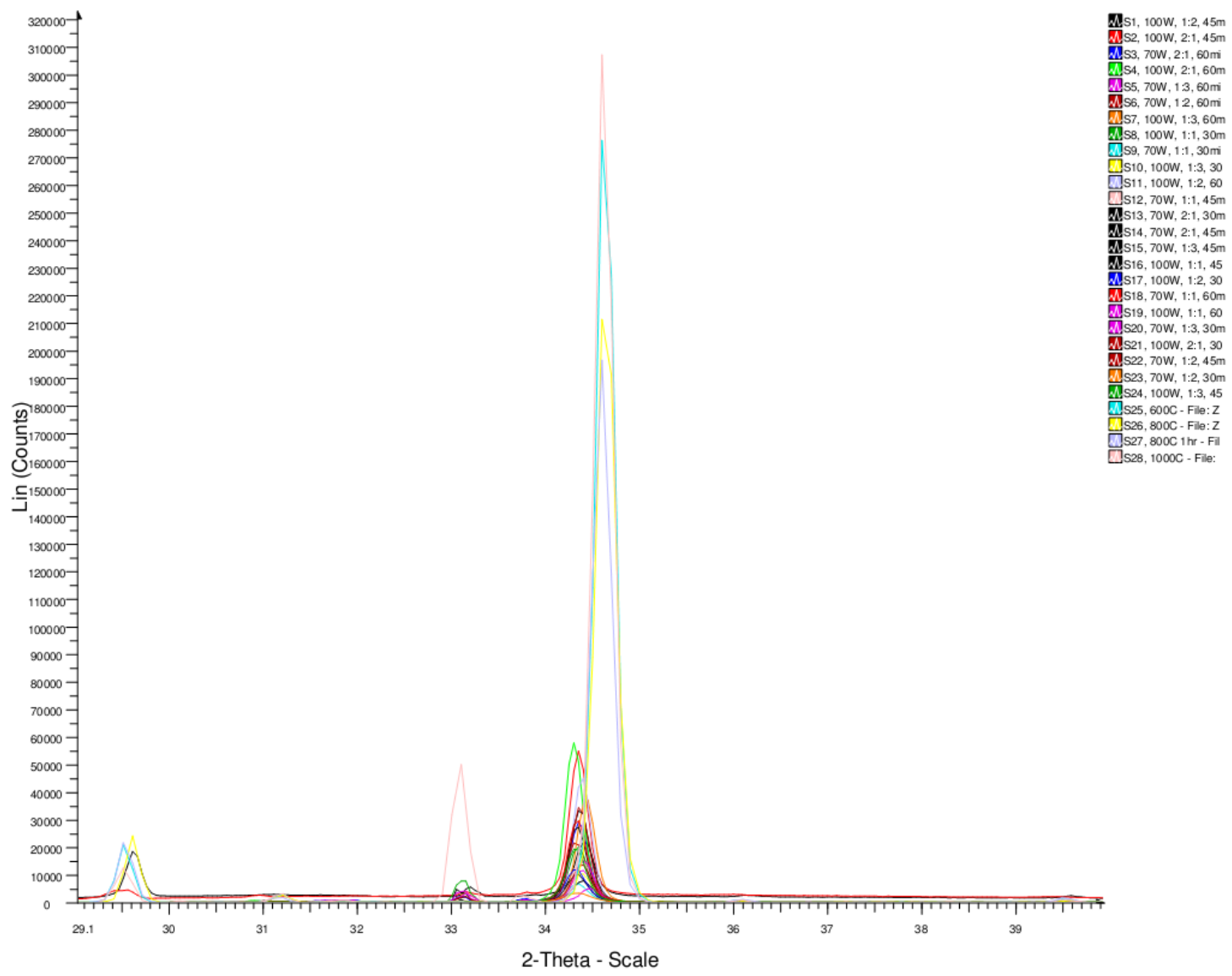

Figure 92 - All 24 as-sputtered samples and the four additional annealed samples are shown in this XRD graph. The four annealed samples had much higher intensities and also showed a shift in the (002) peak angle, indicating relaxation of the crystals.

The effect of the argon to oxygen gas ratios on the structure of the $\mathrm{ZnO}$ was examined for samples sputtered at $100 \mathrm{~W}$ for 60 minutes (Figure 93). The 2:1 gas ratio sample had the highest (002) peak intensity and a low FWHM of $0.220^{\circ}$, which indicates an extremely narrow peak with excellent crystalline quality. The high intensity also indicates that the 2:1 gas ratio features the strongest preferred orientation along the c-axis. Increasing the oxygen partial pressure tends to reduce the intensity of the peaks and also shift the (002) peak angle slightly, but the FWHM values do not seem to follow the same trend. 


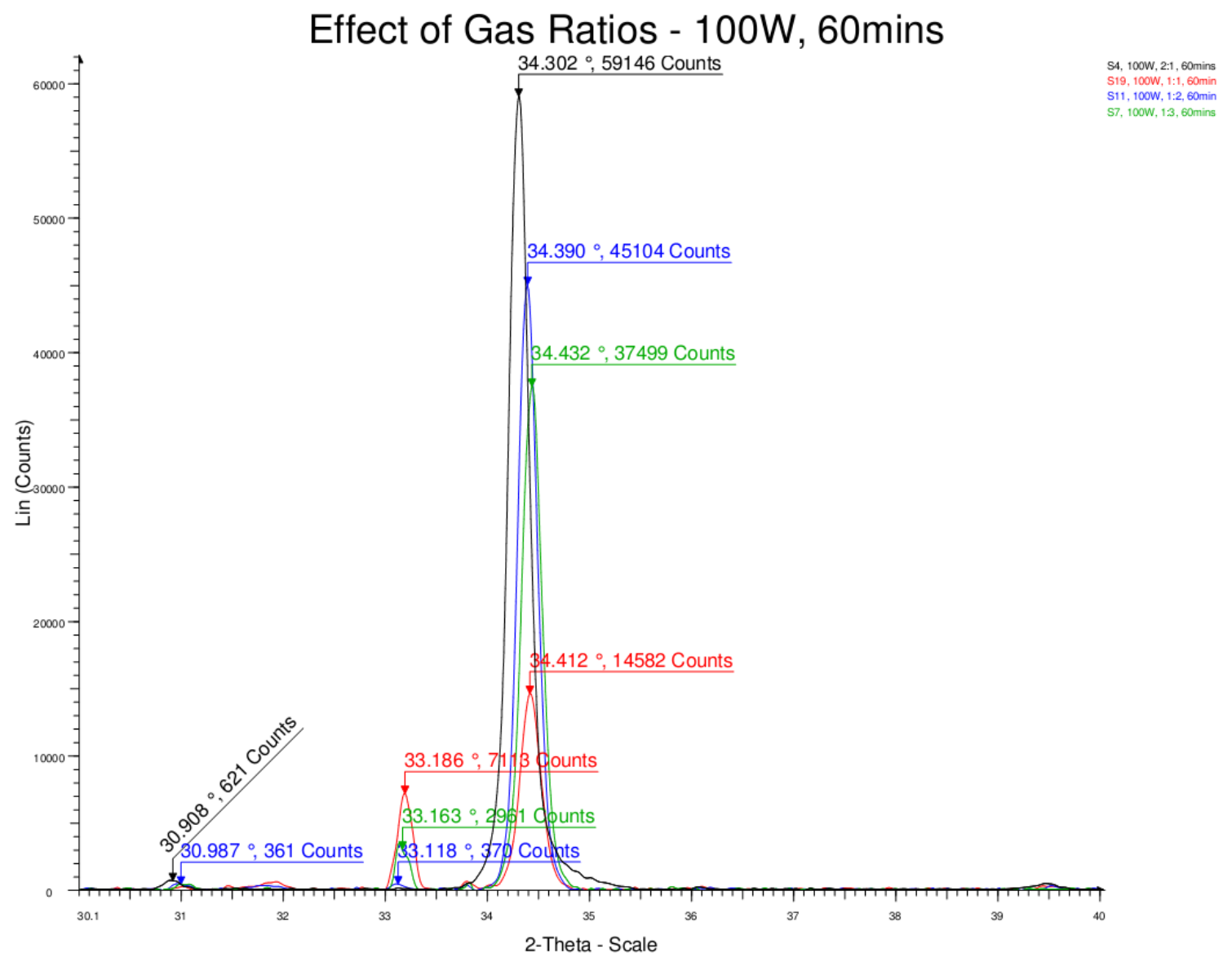

Figure 93 - The 2:1 gas ratio $\mathrm{ZnO}$ sample had the highest intensity (002) peak at $34.302^{\circ}$, indicating that the crystal is strongly c-axis oriented.

The effect of varying the sputtering power on the ZnO's structure, with everything else held constant, was also examined. I found that $100 \mathrm{~W}$ of sputtering power resulting in the highest peak intensities with lower (002) peak angles. Again, the 2:1 ratio samples still had the highest peak intensities out of all of the samples. The samples sputtered with $70 \mathrm{~W}$ of sputtering power resulted in films that had lower (002) peak intensities and were slightly shifted to higher (002) peak angles. Also examined was the effect that sputtering time had on the ZnO's crystalline structure. I found that decreasing the sputtering time also decreases the intensity of the (002) peaks and causes slight variations in the peak angle. Films sputtered for 60 minutes had the highest intensities, followed 
by 45 minutes, and then the lowest intensities at 30 minutes. It seems that there is a correlation between the thickness of the sample and the intensity of the (002) peak angle.

Through the analysis of a DOE for my $24 \mathrm{ZnO}$ samples and a main effects plot, I found that the gas ratio has a small effect on the FWHM of the (002) diffraction peak (Figure 94).The 2:1 gas ratio samples tended to have lower FWHM values than the 1:1,1:2, and 1:3 gas ratios, which indicates that the $2: 1$ $\mathrm{ZnO}$ crystals are of higher quality and are more c-axis oriented. Longer sputtering times and higher sputtering powers also tended to decrease the FWHM. The annealed $\mathrm{ZnO}$ samples had high FWHM values

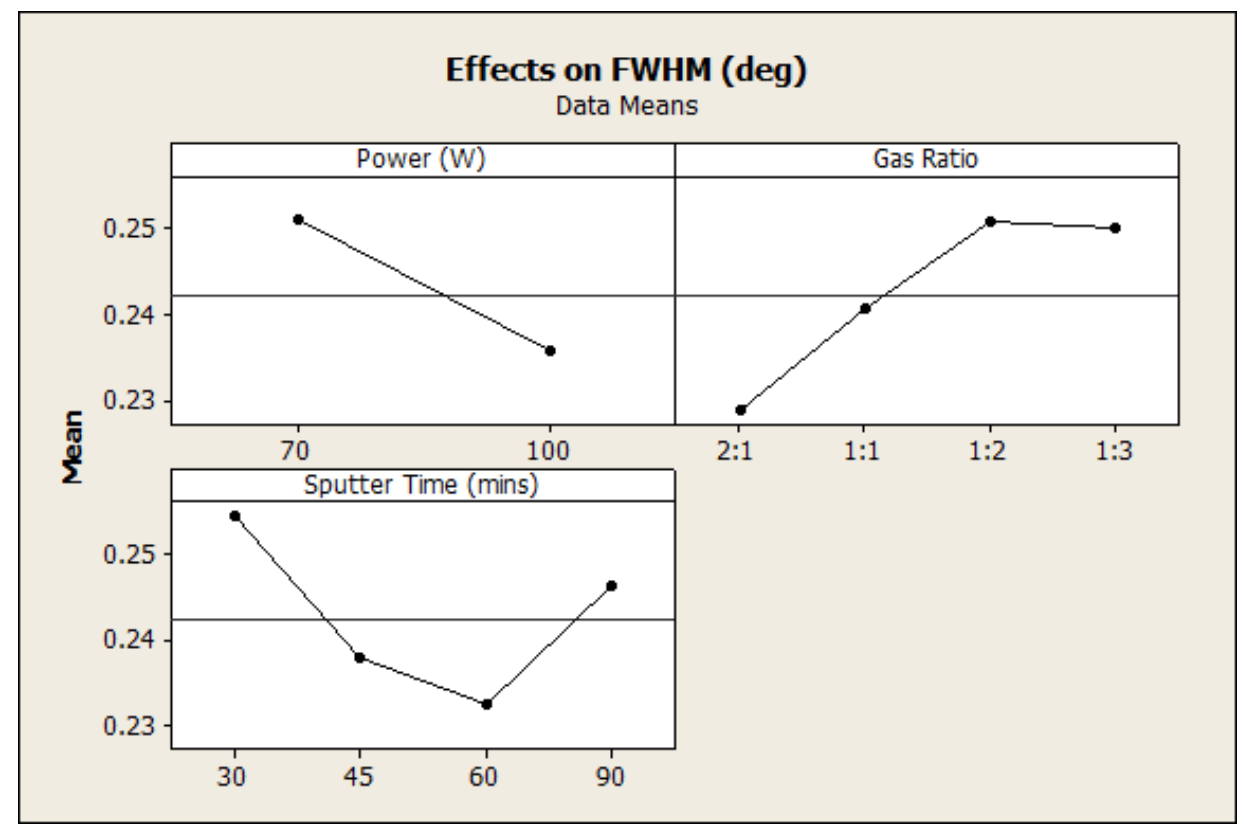

Figure 94 - A graph relating the gas ratios to the FWHM found through XRD measurements shows that as the oxygen partial pressure increases, the FWHM tends to increase as well. The 90 minute sputter represents the four 1:1 ratio, $100 \mathrm{~W}$ annealed $\mathrm{ZnO}$ samples.

\subsubsection{Atomic Force Microscopy (AFM)}

The topography of the $\mathrm{ZnO}$ thin film was obtained using an Asylum Research MFP-3D atomic force microscope. AC mode and AC160TS cantilevers were 
used to obtain this topography image of the $\mathrm{ZnO}$ surface (Figure 95). This technique was not used on all of the samples and was used to get a general idea of the look of the surface. At the surface of this $\mathrm{ZnO}$ sample, which was sputtered for 60 minutes at $100 \mathrm{~W}$ and a gas ratio of $2: 1$, the $\mathrm{ZnO}$ crystals tended to form valleys about 40-50 nm deep. Grains in this sample appear to be between 100$200 \mathrm{~nm}$ in diameter, which is consistent with the results from the SEM.
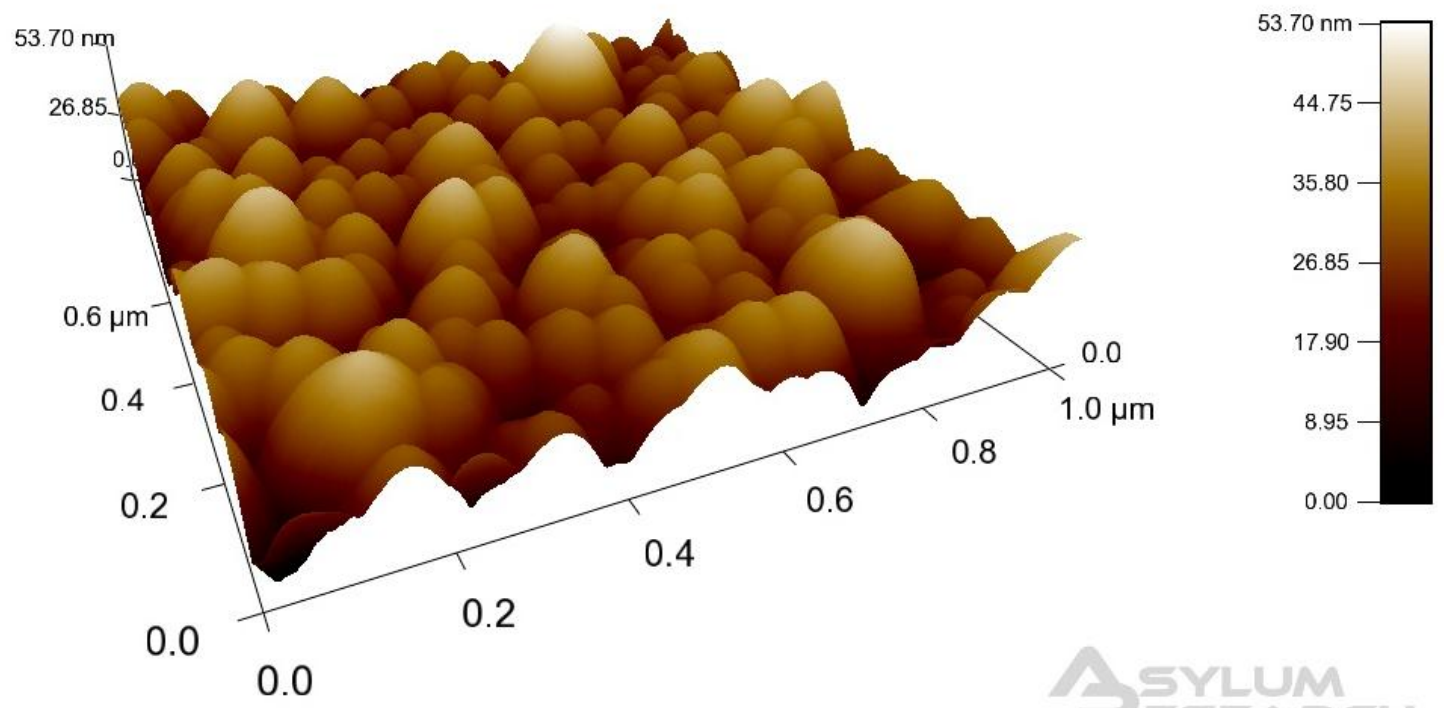

0.0

Figure 95 - The surface topography of a ZnO sample that was sputtered for 60 minutes at $100 \mathrm{~W}$ with a 2:1 gas ratio was obtained using the AC mode on an MFP-3D AFM.

\subsection{Optical Properties}

The optical properties and thicknesses of the $\mathrm{ZnO}$ thin films were determined using a Filmetrics F20 thin-film analyzer, which contains a built-in light source and spectrometer. The reflectometer uses an optical technique called spectral reflectance to gather information about the films thickness and optical constants. It obtains a reflectance spectrum of the material by shining light perpendicular to the surface and analyzing the reflected light (Figure 96). 


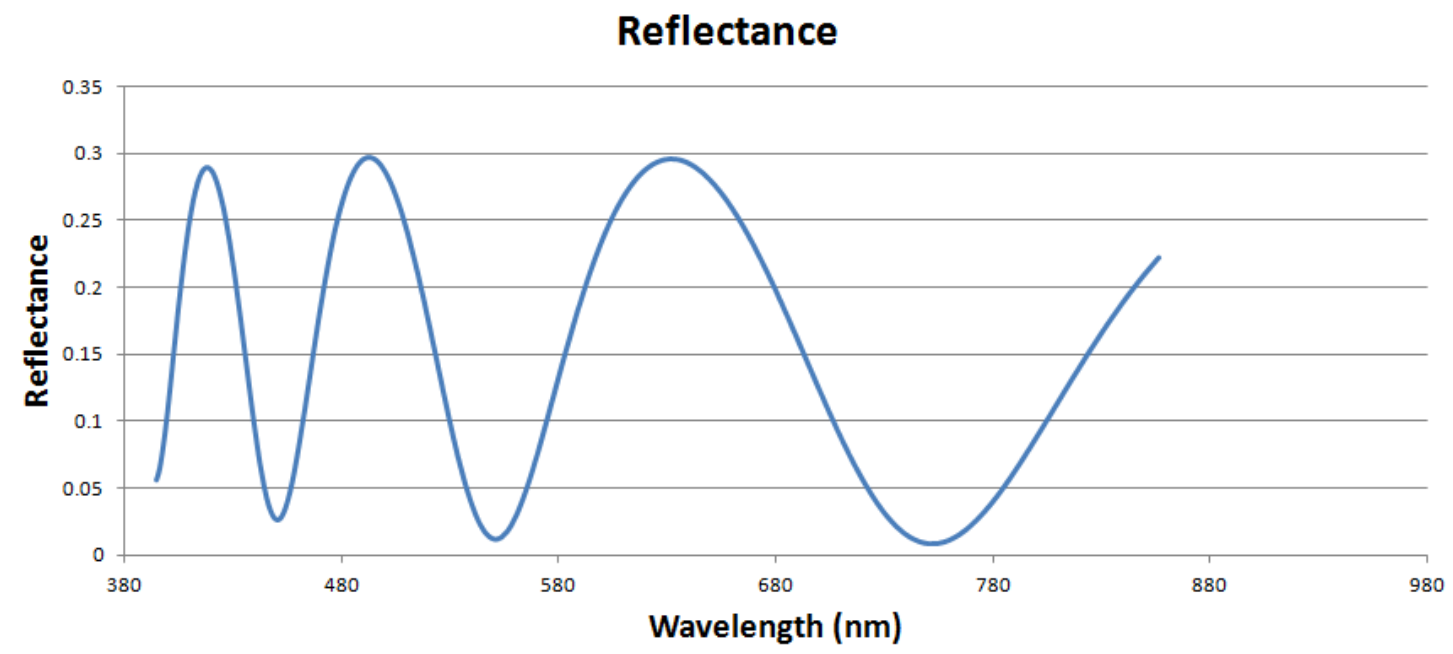

Figure 96 - The reflectance spectrum for a $\mathrm{ZnO}$ sample obtained from a Filmetrics F20 thin-film analyzer.

When measuring the reflectance spectrums of the $\mathrm{ZnO}$ films, the goodness of fit, GOF, of the spectrums should always be as close as possible to 1. A high $\mathrm{GOF}$ is the sign of an extremely accurate measurement. For $\mathrm{ZnO}$, the GOF would sometimes be as low as 0.75 to 0.80 because of several reasons, such as the roughness of the film and film thickness estimates. The presence of slight film surface or interface roughnesses can cause a significant decrease in the GOF measurement, especially if the roughness is above $200 \AA$. When taking the thickness measurements of the $\mathrm{ZnO}$ films, a roughness value was not entered, therefore not allowing the software to account for the roughness of the film. ${ }^{29}$ The GOF could also be affected by improperly estimated initial film thicknesses in the Edit Structure menu. One of the signs of this error is when the periodic oscillations of the measured reflectance spectrum do not match the maxima and minima of the calculated reflectance. Because the initial thickness estimates constrain the software into looking only through a certain range of thicknesses, the FILMeasure software was not able to reach the region of the actual 
thickness. It is useful to know that the thickness of the film increases the number of oscillations in the reflectance spectrums. Another sign that the initial thickness measurements are incorrect are if the measured spectrum has roughly twice the number of oscillations as the calculated spectrum. If this occurs, then it means that the actual film thickness is about twice the thickness of the calculated thickness. ${ }^{29}$ These errors can be avoided by entering in more accurate thickness measurements and roughness measurements or by increasing the range that FILMeasure will analyze.

It has been found that the optical energy band gap is dependent upon the thickness of film, meaning that thinner samples will have higher optical band gaps and thicker samples will have lower band gaps. It is believed that this occurs as a result of differences in grain size, lattice strain, and defect states. ${ }^{35}$

The index of refraction, which describes how light propogates through the $\mathrm{ZnO}$, was determined using the reflectance spectrums found for $\mathrm{ZnO}$ (Figure 97). The index of refraction decreases in an exponential fashion and can range from 2.4 to as low as 1.5 . There does not appear to be any trend that is related to sputtering time and gas ratio. 


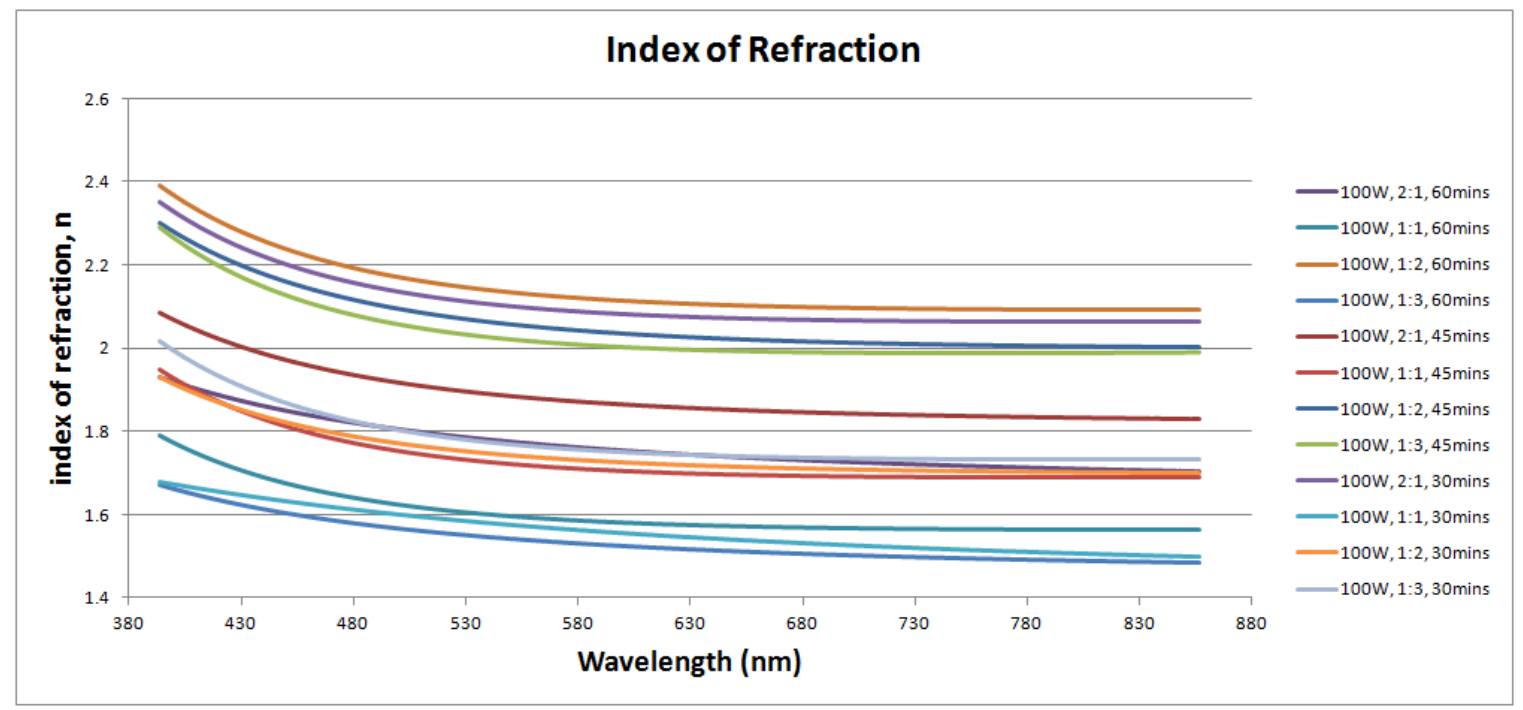

Figure 97 - The index of refraction was determined from reflectance data and the values for all of the $100 \mathrm{~W}$ samples are shown in this graph. The index of refraction tends to decrease exponentially as the wavelength of the light increases.

The extinction coefficients of the $\mathrm{ZnO}$ thin films were also measured and also decrease in an exponential fashion (Figure 98). The extinction coefficient is the imaginary portion of the complex form of the index of refraction and is related to light absorption. The extinction will be used later to calculate the absorption coefficient, which was used to calculate the optical band gap of $\mathrm{ZnO}$.

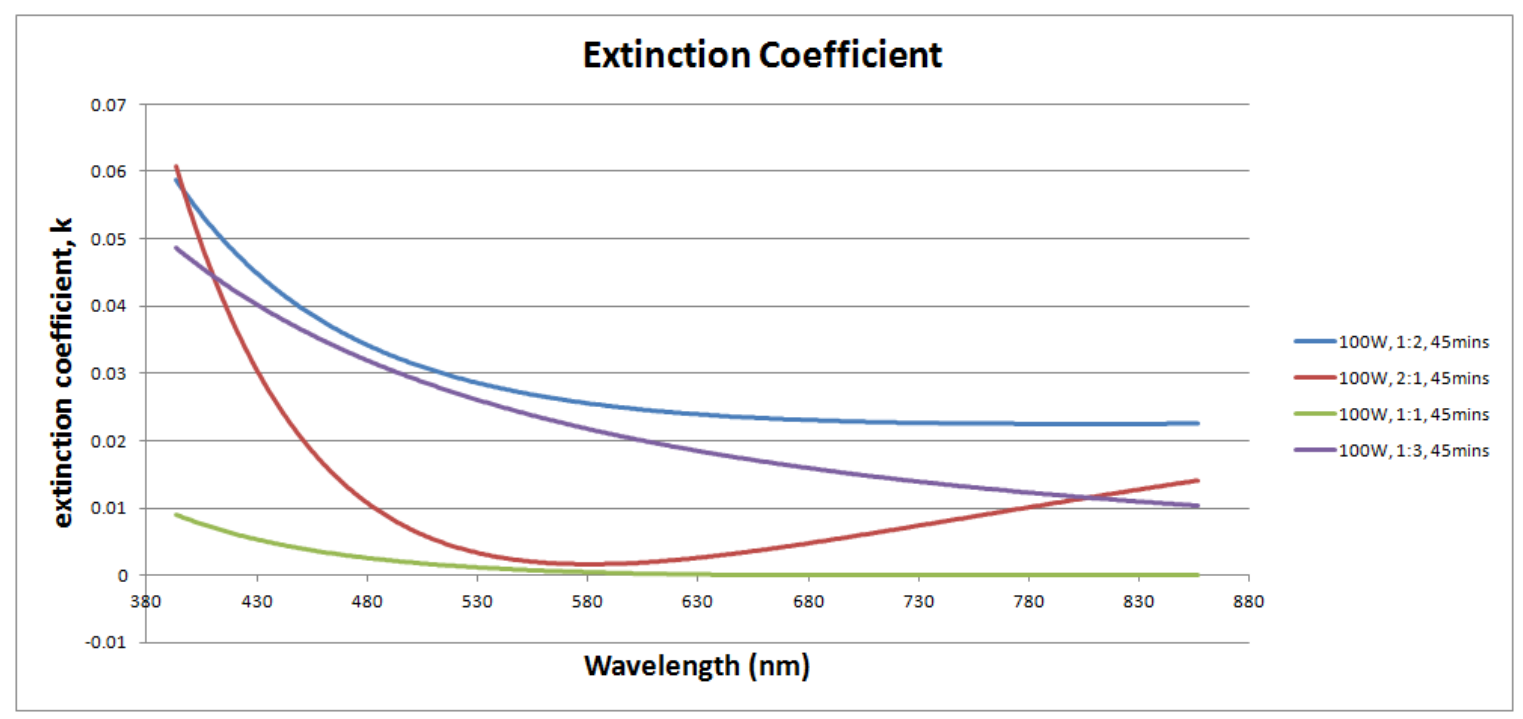

Figure 98 - The extinction coefficient of the as-sputtered $\mathrm{ZnO}$ samples was obtained from reflectance data. 
The optical band gap of the ZnO samples was calculated using the Tauc plot, which is a graph of $h v$ versus $(\alpha h v)^{2}$, where $h$ is Planck's constant, $v$ is the frequency of the emitted photons, and $\alpha$ is the absorption coefficient (Figure 99). The optical band gap is determined by drawing a straight line from the linear portion of the graph until it intersects with the x-axis. The optical band gap of the as-sputtered $\mathrm{ZnO}$ films fluctuated between 2.6 and $2.97 \mathrm{eV}$, with the $2: 1$ gas ratio sputtered ZnO films with the higher band gaps.

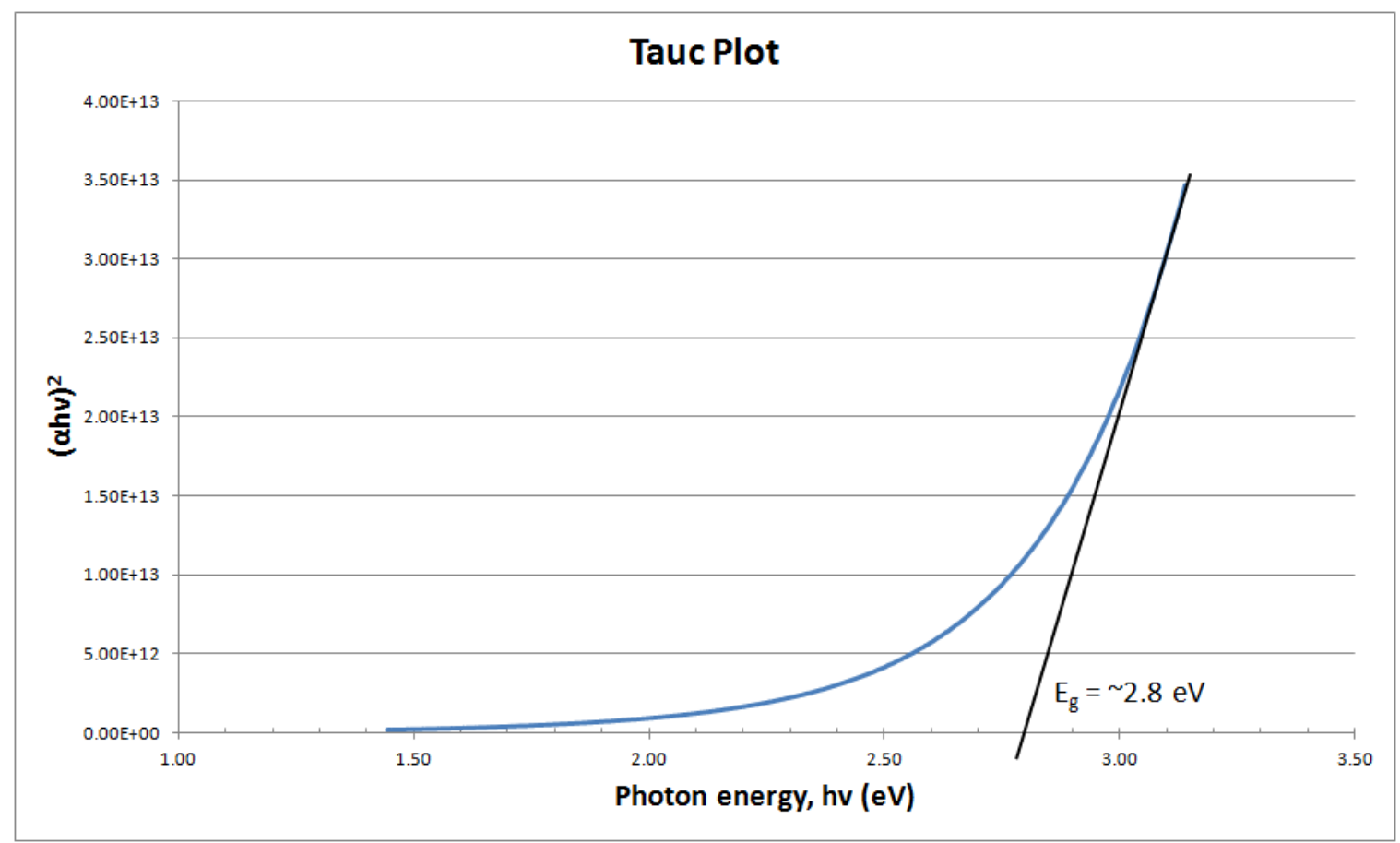

Figure 99 - The Tauc plot is used to calculate the optical band gap of a material by extending the linear portion of the exponential graph to the x-axis. Where the extension hits the x-axis is the band gap of the material.

The annealed samples featured higher level bands gaps, with the $\mathrm{ZnO}$ sample that was annealed at $800^{\circ} \mathrm{C}$ for 1 hour having a band gap of $3.15 \mathrm{eV}$ (Figure 100). The $800^{\circ} \mathrm{C} 1$ hour annealed sample is the only sample with a band gap that is in the range of $3.1-3.3 \mathrm{eV}$, which is typical for $\mathrm{ZnO}$. It is possible for 
$\mathrm{ZnO}$ to have band gaps around $2.8 \mathrm{eV}$, but it tends to require doping. Additional band gap data can be found in Appendix D.

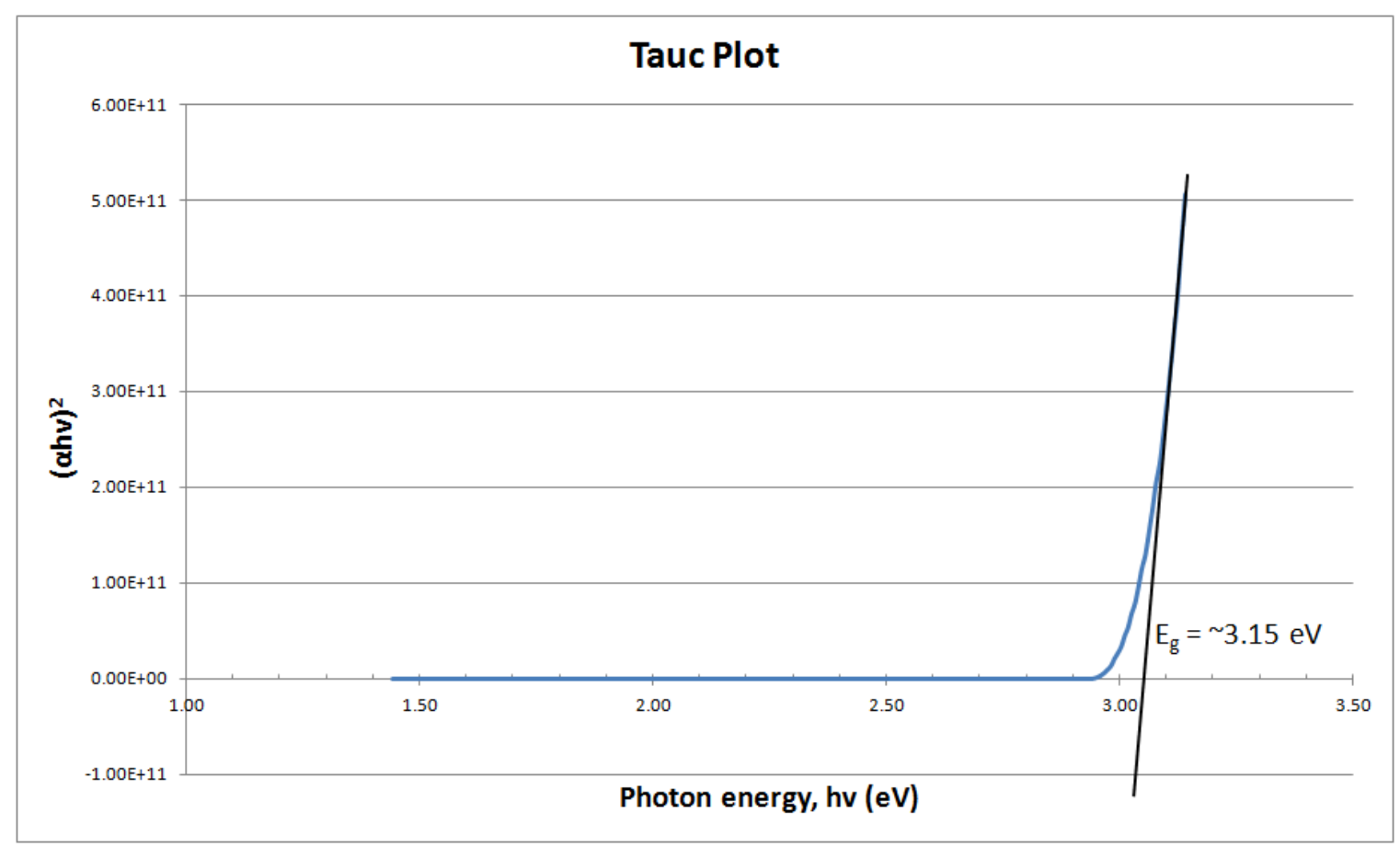

Figure 100 - The band gap for the $\mathrm{ZnO}$ sample annealed at $800^{\circ} \mathrm{C}$ for 1 hour was the only sample that had a band gap in the typical range for $\mathrm{ZnO}$.

\subsection{Electrical Properties}

The electrical resistivity of the $\mathrm{ZnO}$ films was very difficult to measure due to its incredibly insulating nature. Despite trying to measure with extremely low currents, reliable and consistent data could not be obtained for as-sputtered $\mathrm{ZnO}$. A sample of $\mathrm{ZnO}$ was given to Signatone for a high resistivity measurement and they found that the average sheet resistance was about $1.94 \times 10^{9} \Omega$ and the resistivity was approximately $2.6 \times 10^{5} \Omega-\mathrm{cm}$. However, reliable resistivity data of the annealed $\mathrm{ZnO}$ samples could be collected because the annealing increased their electrical conductivity (Figure 101). Additional and more detailed resistivity data can be found in Appendix E. 


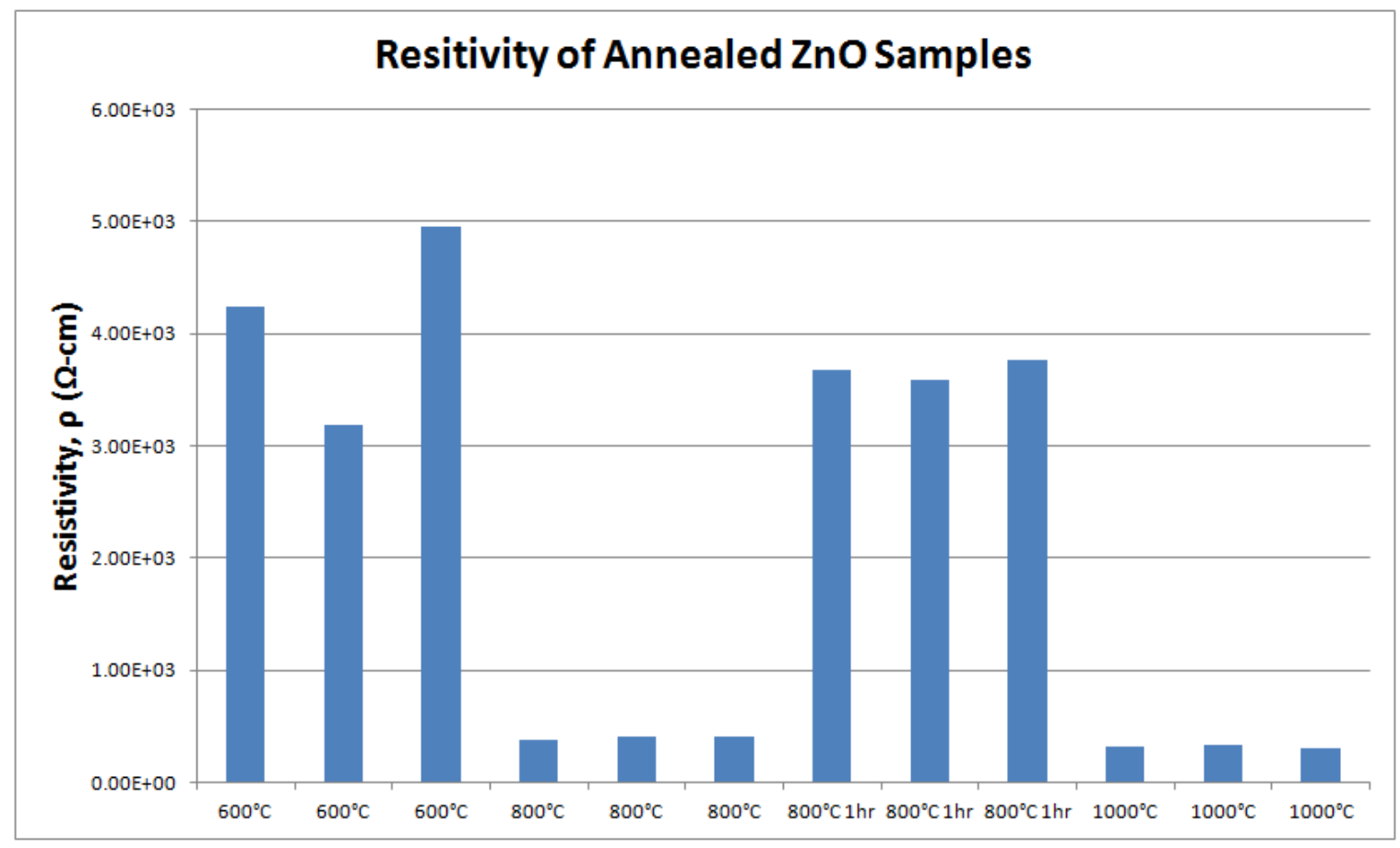

Figure 101 - Unlike the highly resistive as-sputtered $\mathrm{ZnO}$, the resistivity of the annealed $\mathrm{ZnO}$ samples was able to be determined using the 4-point probe method.

It makes sense that the $600^{\circ} \mathrm{C}$ annealed $\mathrm{ZnO}$ sample has the highest resistivity because the voided growth boundaries between the $\mathrm{ZnO}$ crystals have not melded together as much as the other annealed samples. The voided boundaries between the $\mathrm{ZnO}$ crystals prevent charge from transferring between crystals, thus making its resistivity high. The high resistivity of the $\mathrm{ZnO}$ sample that was annealed at $800^{\circ} \mathrm{C}$ for 1 hour is somewhat of an anomaly. The resistivity should be even lower than the sample annealed $800^{\circ} \mathrm{C}$ for 30 minutes. Judging by the way that the sample looked in the cross-sectional SEM picture, it seems as though the crystals were not able to diffuse into each other like they did with the 30 minute $800^{\circ} \mathrm{C}$ and $1000^{\circ} \mathrm{C}$ anneals.

Resistive heating was a major problem when initially attempting to measure the resistivity of the $\mathrm{ZnO}$ films. At the time, I did not realize that supplying 1-10 
$\mathrm{mA}$ of direct current would cause incredible amounts resistive heating to occur. The amount of resistive heating, also known as Joule heating, can be measured with Joule's first law (Equation 11).

$$
Q=I^{2} \cdot R \cdot t
$$

Equation 11 - Joule's first law relates the amount of heat to the amount of current flowing through a conductor for a certain amount of time.

Where $Q$ is the heat generated [J, joules], I is a constant current being applied to the conductor [A, amperes], $R$ is the electrical resistance of the conductor $\left[\Omega, \frac{J}{s \cdot A^{2}}\right]$, and $t$ is time [seconds]. Since the resistance of the $\mathrm{ZnO}$ thin films is extremely high and on the order of mega-ohms, when 1-10 mA of current is supplied to the film, it results in large amounts of heat. Since Joule's first law is also a function of time, the longer the measurement is taken, the more heat that is generated. If the current is reduced to nano-amps or micro-amps, this heat is significantly lower and the risk of damaging and melting the Delrin probe heads is greatly reduced. These Delrin probe heads are only specified to handle temperatures up to $90^{\circ} \mathrm{C}$ and temperatures any higher will melt and ruin the probe head. In order to measure the high resistances of the $\mathrm{ZnO}$ thin films, a high temperature and high resistance probe head would need to be used. These high resistance probe heads are made of Macor and possess higher spring pressures of 180 grams. Although this is not the case for $\mathrm{ZnO}$, the high spring pressures are typically used because high resistivity materials like silicon can often naturally form non-conductive layers when exposed to the atmosphere and if you want to measure the resistivity of the silicon and not the oxide, then the probe tip must break through the oxide. For $\mathrm{ZnO}$, a high resistance probe head 
would allow higher currents to be supplied, which are required to get stable and repeatable voltage measurements. Too little current supplied to the film and the voltage measurements will fluctuate and change dramatically during measurements. The reason voltage measurements fluctuate and become unstable can be explained through Ohm's law (Equation 12).

$$
V=I R
$$

Equation 12 - Ohm's law is a simple explanation as to why the lower the current supplied, the lower the measured voltages.

Where $V$ is measured voltage [volts], $I$ is the supplied current [amperes], and $R$ is the resistance of the thin film $[\Omega$, ohms]. If very little current is being supplied to the film and incredibly large amounts of resistance are present, then the voltage measurements will be difficult to measure. To measure these extremely low voltage values a higher quality digital multimeter could be used. According to Ohm's law, when more current is supplied to the thin film, the voltage measurement will increase as well and make the measurement more stable.

The SP4 probe head is a unique tool that can be completely customized based on the application at hand. To be able to measure the electrical properties of these $\mathrm{ZnO}$ films, a more accurate digital multimeter might need to be acquired, or the SP4 probe head parameters could be changed. For example, the spacing between the tips can be changed to 0.0625 inches (62), 0.050 inches (50), or 0.040 inches (40) apart. The probe tip spacings are selected depending on the sample geometry and how small the samples are and how close to the edge of the device needs to be measured. Larger tip spacings will generally give better results. The pressure on each probe tip can vary between 45 grams (045), 85 
grams (085), and 180 grams (180). Differing pressures are offered because ohmic or electrical contact needs to be made with the surface and if there are native oxides in the way of the target's surface then the probe tips need to pierce through the oxide. The tip pressure will depend on the hardness of the film, with soft films (like metal) requiring small pressures (45 g), and hard, nonconductive films requiring higher pressure (180 g). Signatone recommends using $85 \mathrm{~g}$ for intermediate or unknown films. The radius of the tip can vary between 0.00016 inches (1.6 mil), 0.005 inches $(5 \mathrm{mil})$, and 0.010 inches $(10 \mathrm{mil})$, with the $1.6 \mathrm{mil}$ tip radius being the standard size. The larger radius tips are more robust, but are more difficult to make good electrical contact with the film. For films and thin films that are easily contacted, Signatone recommends a 5 mil tip radius. For extremely thin films, they recommend using a 10 mil radius tip. Additionally, the probe tip material can either be Osmium (O) or Tungsten Carbide (T). Osmium is a hard amorphous material that is more forgiving to slight horizontal motions than tungsten carbide. Osmium is slightly more expensive than the tungsten carbide tips, but should last longer and provide more touchdowns of the four-point probe. Osmium can also make better contact with exotic materials because of its unique work function. ${ }^{51}$ Tungsten carbide is an extremely hard and wear resistant carbide that is formed by sintering tungsten metal powder and carbon in a hydrogen environment and pressing them into desired shapes. ${ }^{52}$ The tungsten carbide provides the tips with excellent electrical conductivity and wear resistance, but because of tungsten carbide's hardness, it is brittle and susceptible to failure with horizontal movements. Tungsten carbide tips are used 
for the wear resistance and are usually found in low-volume laboratory environments.

\subsection{Piezoelectric Properties}

The piezoelectric properties of the $\mathrm{ZnO}$ thin films were determined using an AFM technique called piezoresponse force microscopy (PFM). PFM can measure the local piezoelectric properties of thin films by applying a voltage to the piezoelectric sample through a conductive cantilever. Using this technique which was described in the processing section, the piezoelectric coefficient, $d_{33}$ was determined for the as-sputtered and annealed $\mathrm{ZnO}$ samples (Figure 102). More detailed $d_{33}$ data can be found in Appendix C.

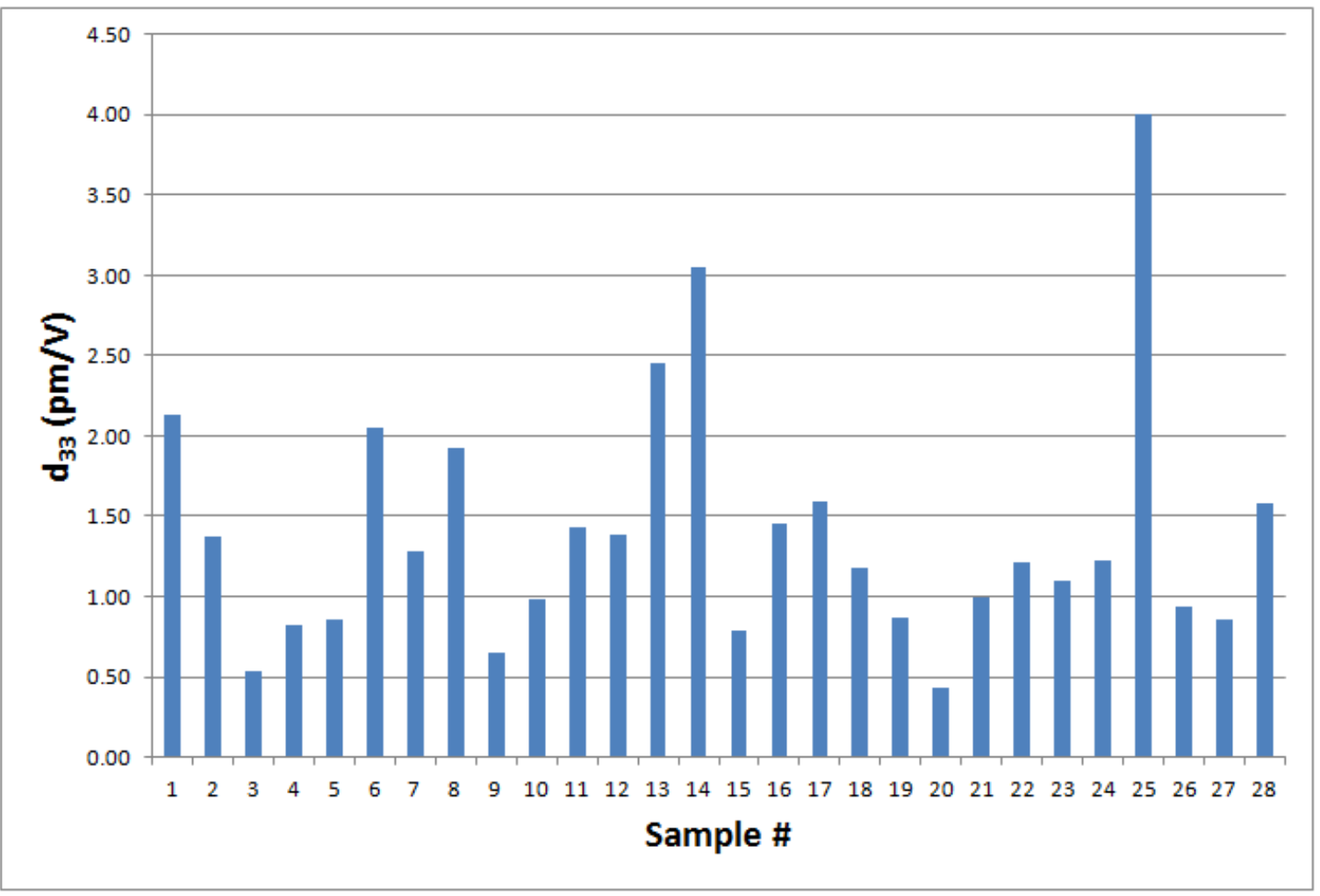

Figure 102 - The $d_{33}$ values were calculated for all of the as-sputtered and the four annealed samples. The sample annealed at $600^{\circ} \mathrm{C}$ for 30 minutes resulted in the highest piezoelectric response. 
Out of all of the as-sputtered samples, the sample that was sputtered for 45 minutes at $70 \mathrm{~W}$ and with a gas ratio of 2:1 resulted in the highest piezoelectric coefficient of $3.05 \mathrm{pm} / \mathrm{V}$. However, the $600^{\circ} \mathrm{C}$ annealed $\mathrm{ZnO}$ sample, which was sputtered with a 1:1 gas ratio, 100W, and for 90 minutes, resulted in the highest $d_{33}$ out of all of the samples at $4.00 \mathrm{pm} / \mathrm{V}$.

The highest $d_{33}$ values seemed to result from the 2:1 gas ratio samples sputtered at low powers (Figure 103). The films sputtered with a 1:3 gas ratio had the worst piezoelectric response. Surprisingly, many of the thicker samples that were sputtered at $100 \mathrm{~W}$ and with 2:1 gas ratios did not exhibit good piezoelectric responses. This is surprising because it seemed like from the XRD measurements, the samples with higher intensity (002) peaks and lower FWHM values would have had the best piezoelectric response because of their excellent crystalline quality, but that was not the case. The 2:1 gas ratio samples might have poor piezoelectric properties because the $\mathrm{ZnO}$ films contain a higher percentage of zinc than in the other samples, making the $\mathrm{ZnO}$ more metallic in nature. The thick $\mathrm{ZnO}$ thin films may have poor piezoelectric properties because the films will experience more stresses the thicker the film becomes, which can distort the crystal lattice. 


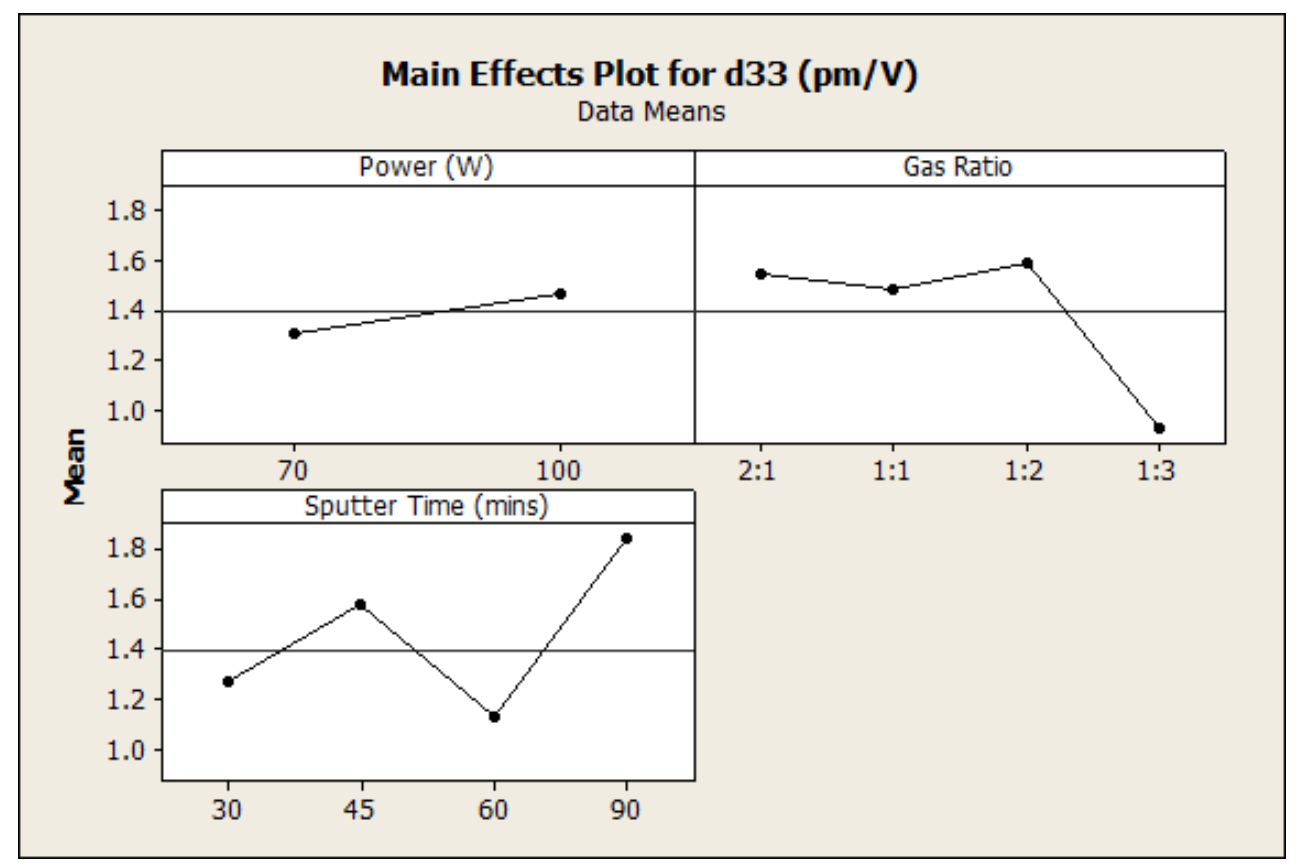

Figure 103 - A main effects plot was generated from Minitab and the data from the 24 as-sputtered $\mathrm{ZnO}$ samples and the four annealed $\mathrm{ZnO}$ samples. High oxygen partial pressures tended decrease the piezoelectric response of the $\mathrm{ZnO}$. A 90 minute sputter was performed only on the four annealed samples.

When looking at the $d_{33}$ value for the $600^{\circ} \mathrm{C}$ annealed sample, it makes a lot of sense that it would have the highest piezoelectric coefficient. The low temperature annealing allowed the crystal to come to a relaxed state, but did not heat up enough to cause the voided growth boundaries to close between each crystal. The piezoelectric response of the other annealed samples was extremely poor, most likely because the voided boundaries that separated the individual crystals disappeared due to the high temperatures of the furnace. It seems that a more relaxed crystal lattice results in a better piezoelectric response. If the $\mathrm{ZnO}$ crystals have both a relaxed crystal lattice and voided growth boundaries between the crystals then the piezoelectric response will be highest.

To determine the effects of performing the scans on the tip of the conductive cantilevers, SEM micrographs were taken of the tip before and after scanning (Figure 104). After only a few PFM scans, the conductive tip of the AC240TM 
ElectriLevers experienced a significant amount of dulling and degradation. To obtain accurate PFM measurements, the conductive cantilevers should only be used a few times or until the PFM images and signals start to degrade.

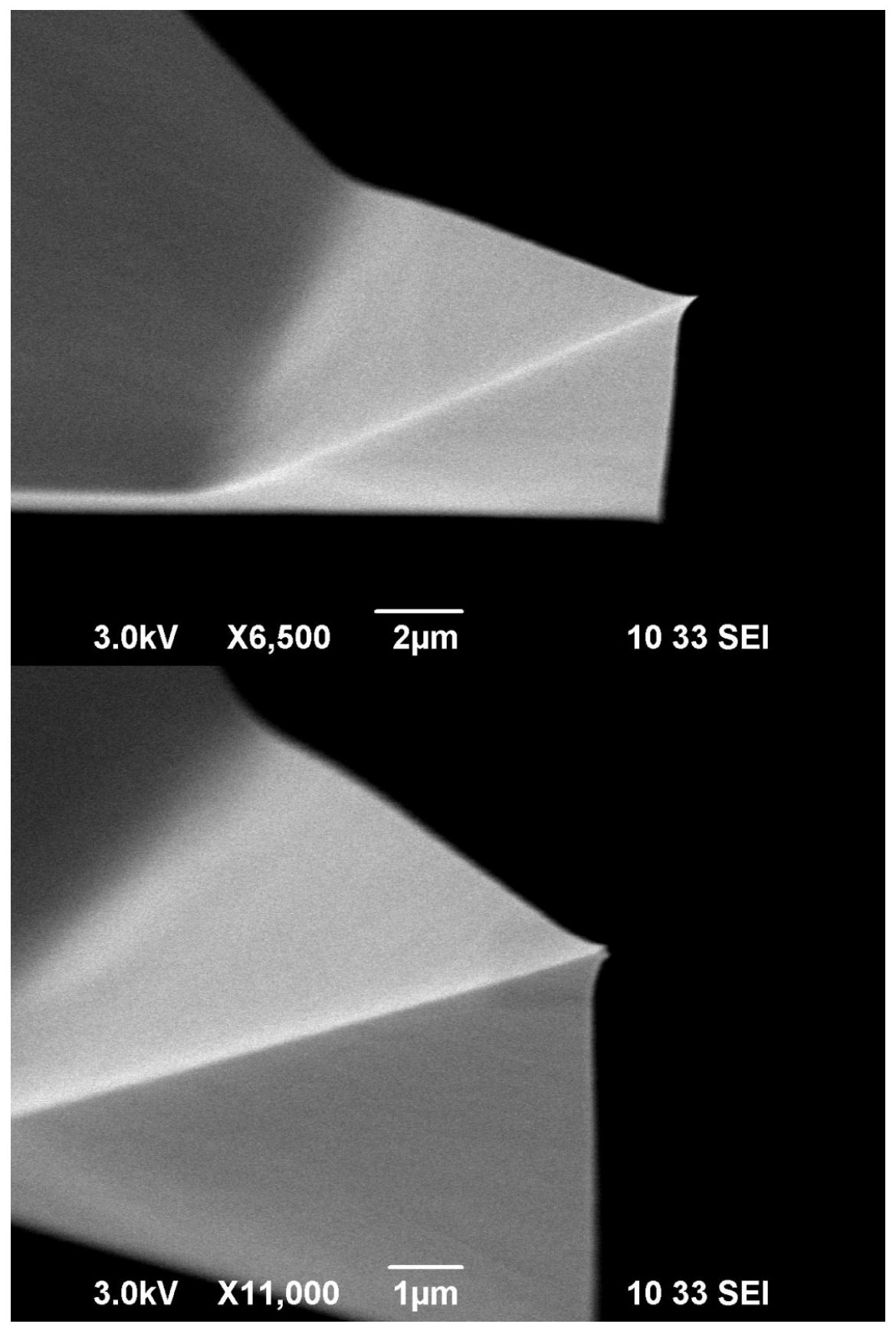

Figure 104 - The conductive tips experienced severe degradation after only a few scans. The top SEM micrograph shows an image of an unused conductive tip and the bottom micrograph shows the same tip after only a few PFM scans. 


\section{Chapter 4: Conclusions}

A piezoelectric microspeaker device was fabricated, but in the end did not function properly because of several problems that were encountered during the fabrication process. The main obstacle in the fabrication process was wet etching a thin diaphragm in the silicon substrate. The etch rate of the TMAH would often fluctuate and change across wafers and during the wet etching process itself. A better way to etch the silicon is required or a more flexible diaphragm material like parylene could be used instead. ${ }^{53}$ In literature, others have etched through all of the silicon and used a more flexible and thinner support structure such as silicon nitride or parylene for the $\mathrm{ZnO}$ film. ${ }^{46}$ Another problem that could have resulted in the non-functioning device is the clamping effect that the rigid silicon substrate has on the thin $\mathrm{ZnO}$ film. With a more flexible diaphragm, this problem could be avoided as well. However, this was more encouraging than discouraging because it meant that more information needed to be known about the sputtered $\mathrm{ZnO}$ films. Without knowledge of the ZnO's structural properties, optical properties, electrical properties, and piezoelectric properties, a working device was difficult to fabricate.

The structural properties of the $\mathrm{ZnO}$ thin films were investigated with scanning electron microscopy, x-ray diffraction, profilometry, and atomic force microscopy. Through cross-sectional images of the $\mathrm{ZnO}$ from the SEM, the film thicknesses of the thin films were determined. SEM images also showed $\mathrm{ZnO}$ with distinct columnar structures with voided growth boundaries between the crystals. The grain sizes were also determined from the topographical images of the $\mathrm{ZnO}$. 
Annealed $\mathrm{ZnO}$ samples were also investigated and it was found that they underwent significant changes in their crystals through a sintering-like process of diffusion. X-ray diffraction (XRD) was utilized on the $\mathrm{ZnO}$ samples as well and it was found that the crystals are highly c-axis oriented in the (002) crystal plane. The FWHM values for each of the samples were also determined from the XRD measurements, which gives insight into the crystalline quality of the $\mathrm{ZnO}$. The ZnO samples that were sputtered with a 2:1 gas ratio tended to sputter the fastest and thus had the thickest films. The 2:1 gas ratio samples also hadthe best crystalline quality, but resulted in $\mathrm{ZnO}$ films that were more metallic in nature. $\mathrm{ZnO}$ samples with a 1:3 gas ratio sputtered the slowest and had the lowest FWHM values and therefore low crystalline quality. The AFM was also used briefly on some $\mathrm{ZnO}$ samples to confirm grain sizes and the topographical structure of the $\mathrm{ZnO}$. The film thicknesses of the $\mathrm{ZnO}$ samples were determined using SEM, profilometry, and through reflectance data and were found to be similar in the thicknesses. More detailed data about the $\mathrm{ZnO}$ thicknesses can be found in Appendix B.

The optical properties of the $\mathrm{ZnO}$ were discovered using a Filmetrics F20 thinfilm analyzer, which uses spectral reflectance to measure optical constants and the thickness of the $\mathrm{ZnO}$ films. The thicknesses were verified against the SEM data and the profilometer data and were found to be consistent, as shown in Appendix $\mathrm{B}$. The band gaps of the $\mathrm{ZnO}$ were calculated using the Tauc plot and ranged from 2.6 $-2.97 \mathrm{eV}$ for the as-sputtered $\mathrm{ZnO}$ films. However, the annealed samples had higher band gap energies, with a $\mathrm{ZnO}$ sample that was annealed at 
$800^{\circ} \mathrm{C}$ for 1 hour having a band gap energy of $3.15 \mathrm{eV}$, which is in the range of typical values for non-doped $\mathrm{ZnO}$.

The electrical properties of the $\mathrm{ZnO}$ samples were determined with a Signatone SP-2 four-point probe stand and a Signatone SP4 probe head. The as-sputtered $\mathrm{ZnO}$ samples possessed resistivities that were too high for the fourpoint probe and the Keithley 2400 SourceMeter to measure, but the resistivity of the annealed samples were determined. The annealing process increased the electrical conductivity of the $\mathrm{ZnO}$ and allowed the resistivity to be measured consistently with repeatable measurements. Before the annealing process, the resistivity of the as-sputtered $\mathrm{ZnO}$ films could not even be measured with the equipment in Cal Poly's Microfabrication Lab.

Finally, the piezoelectric properties of the $\mathrm{ZnO}$ were discovered using an Asylum MFP-3D and the DART piezoresponse force microscopy (PFM) mode. The PFM mode measures piezoelectric properties by applying a voltage to the surface of the piezoelectric through a conductive cantilever. When the piezoelectric material responds to the voltage with a displacement, the cantilever can detect the change in height. The piezoelectric coefficient, $d_{33}$, was calculated using the DART-PFM method and it was found that the highest as-sputtered piezoelectric coefficient came from a $\mathrm{ZnO}$ sample that was sputtered with low sputtering power and a 2:1 gas ratio. The highest $d_{33}$ value for an as-sputtered sample was $3.05 \mathrm{pm} / \mathrm{V}$. The highest piezoelectric coefficient of $4.0 \mathrm{pm} / \mathrm{V}$ was seen with the $600^{\circ} \mathrm{C}$ annealed $\mathrm{ZnO}$ sample. This was accomplished because the annealing helped relax the stresses in $\mathrm{ZnO}$ crystal lattices caused by the 
deposition process, but it was not hot enough to cause the voided growth boundaries to diffuse together and disappear entirely. Above $600^{\circ} \mathrm{C}$, the annealing caused the piezoelectric response of the $\mathrm{ZnO}$ to diminish.

\section{Chapter 5: Future Work}

Now that extensive characterization techniques have been performed on the sputtered $\mathrm{ZnO}$ thin films, we are closer to being able to fabricate a working piezoelectric microspeaker in the Cal Poly Microfabrication Lab. However, it is difficult to predict whether a functional $\mathrm{ZnO}$ microspeaker can be fabricated because of the difficulty in modeling the behavior between the $\mathrm{ZnO}$ and the silicon diaphragm. In addition, this discovery process of $\mathrm{ZnO}$ 's properties was more of a survey to find out where more research and characterization should be performed. From the extremely promising results that I have seen from annealing $\mathrm{ZnO}$ films, it seems like annealed $\mathrm{ZnO}$ could be a way to achieve stronger piezoelectric responses. It would be worth investigating the effects of annealing more thoroughly. More annealing temperatures and annealing times should be attempted and their effects on the piezoelectric properties investigated. Turning $\mathrm{ZnO}$ into a transparent conductive oxide (TCO) is another possibility that could be investigated in the future. This will be achieved either through an annealing process or through doping of the $\mathrm{ZnO}$. Doping of $\mathrm{ZnO}$ is extremely common and well-documented and can be performed by DC reactive sputtering with an aluminum target and a zinc target, resulting in a band gap widening of $\sim 0.5 \mathrm{eV} .{ }^{54}$ From here, there should be plenty of new and innovative future work that can be accomplished with zinc oxide. 


\section{List of References}

${ }^{1}$ Marek, Jiri, and Udo-Martin Gomez. "MEMS (Micro-Electro-Mechanical Systems) for Automotive and Consumer Electronics." Chips 2020 A Guide to the Future of Nanoelectronics. By Bernd Hoefflinger. Berlin, Heidelberg: Springer-Verlag Berlin Heidelberg, 2012. 293-313. Print.

2 "Sustainability Report 2009." East Japan Railway Company. 2009. Web. 17 Jan. 2012. <http://www.jreast.co.jp/e/environment/2009.html>.

${ }^{3}$ Hughes, William. "Synthesis and Characterization of Zinc Oxide Nanostructures for Piezoelectric Applications." Dec. 2006. Print.

${ }^{4}$ IEEE Standard on Piezoelectricity, IEEE Standard 176, 1987.

${ }^{5}$ Piezoelectric Ceramics: Principles and Applications. Mackeyville, PA: APC International, 2002. Print.

6 "Piezoelectric Constants." APC International, Ltd. Web. 26 Mar. 2012. $<$ http://www.americanpiezo.com/knowledge-center/piezo-theory/piezoelectric-constants.html>.

${ }^{7}$ Ellmer, Klaus, Andreas Klien and Bernd Rech. "ZnO and Its Applications." Transparent Conductive Zinc Oxide. Springer, 2008. 1-33.

8 "The Wurtzite (B4) Structure." Center for Computational Materials Science. Web. 24 Feb. 2012. <http://cst-www.nrl.navy.mil/lattice/struk/b4.html>.

${ }^{9}$ Streetman, Ben G., and Sanjay Kumar Banerjee. Solid State Electronic Devices. London: Prentice-Hall, 2006. Print.

${ }^{10}$ Leon M. Tolbert, Burak Ozpineci, S. Kamrul Islam, Madhu Chinthavali. "Wide Bandgap Semiconductors for Utility Applications." IASTED International Conference on Power and Energy Systems (PES 2003), February 24-26, 2003, Palm Springs, California, 317-321.

${ }^{11}$ Kenanakis, G., M. Androulidaki, E. Koudoumas, C. Savvakis, and N. Katsarakis. "Photoluminescence of $\mathrm{ZnO}$ Nanostructures Grown by the Aqueous Chemical Growth Technique." Superlattices and Microstructures 42.1-6 (2007): 473-78. Print.

${ }^{12}$ Rosenbaum, J. F. Bulk Acoustic Wave Theory and Devices. Norwood, MA: Artech House, 1945. Print. 
${ }^{13}$ Franz, Gerhard. "Plasma Deposition Processes." Low Pressure Plasmas and Microstructuring Technology. 2009. 2 Nov. 2011. 375-438. Web.

${ }^{14}$ Schuler, Leo P. Properties and Characterisation of Sputtered ZnO. Thesis. University of Canterbury, 2008. UC Research Depository. Web. 18 Nov. 2011.

<http://ir.canterbury.ac.nz/handle/10092/1900>.

${ }^{15}$ Kappertz, O., R. Drese, J. M. Ngaruiya, and M. Wuttig. "Reactive Sputter Deposition of Zinc Oxide: Employing Resputtering Effects to Tailor Film Properties." Thin Solid Films484.1-2 (2005): 64-67. ScienceDirect. Elsevier, 13 May 2005. Web. 18 Jan. 2012. <http://www.sciencedirect.com/science/article/pii/S0040609005002439>.

${ }^{16}$ Szyszka, B. "Magnetron Sputtering of ZnO Films." Transparent Conductive Zinc Oxide. Vol. 104. Springer, 2008. 187-233. Springer Ser. in Materials Science.SpringerLink. Springer, 2008. Web. 14 Oct. 2011. <http://www.springerlink.com/content/p2k050345860710n/>.

${ }^{17}$ Kasap, Safa, and Peter Capper. "Thin Films." Springer Handbook of Electronic and Photonic Materials. Springer US, 2007. 659-716.

${ }^{18}$ Schuler, Leo et al. "Comparison of DC and RF Sputtered Zinc Oxide Films with Post-Annealing and Dry Etching and Effect on Crystal Composition." Japanese Journal of Applied Physics 44.10 (2005): 7555-7560. Print.

${ }^{19}$ Sawyer, Linda C., David T. Grubb, and Gregory F. Meyers. "Fundamentals of Microscopy." Polymer Microscopy. New York, NY: Springer, 2008. 27-66. Print.

${ }^{20}$ Hawkes, Peter W., and John C. H. Spence. Science of Microscopy. New York (NY): Springer, 2007. Print.

${ }^{21}$ Wang, Zhong Lin., and Weilie Zhou. Scanning Microscopy for Nanotechnology: Techniques and Applications. New York, NY: Springer, 2007. Print.

22 Sôrby, Magnus H. "Status on Existing Technologies: Powder Diffraction." Hydrogen Technology: Mobile and Portable Applications. By Aline Léon. Berlin: Springer, 2008. 523-48. Print. 
${ }^{23}$ Wang, Shyh. "Crystal Diffraction." Fundamentals of Semiconductor Theory and Device Physics. Englewood Cliffs, NJ: Prentice Hall, 1989. 52-57. Print.

${ }^{24} \mathrm{Li}$, Dongqing. "X-Ray Diffraction (XRD)." Encyclopedia of Microfluidics and Nanofluidics. New York: Springer, 2008. 2197-199. Print.

${ }^{25}$ Banerjee, A., C. Ghosh, K. Chattopadhyay, H. Minoura, A. Sarkar, A. Akiba, A. Kamiya, and T. Endo. "Low-temperature Deposition of ZnO Thin Films on PET and Glass Substrates by DCsputtering Technique." Thin Solid Films 496.1 (2006): 112-16. Print.

${ }^{26}$ Czichos, Horst, T. Saito, and L. R. Smith. "Surface and Interface Characterization." Springer Handbook of Materials Measurement Methods. Berlin, Germany: Springer, 2006. 230-72. Print.

${ }^{27}$ Larsen-Basse, Jorn. "Basic Theory of Solid Friction." Friction, Lubrication, and Wear

Technology. Vol. 18. ASM International, 1992. ASM Handbooks Online. ASM Handbooks Online. ASM International, 1997. Web. 5 Apr. 2012. <http://products.asminternational.org/hbk>.

${ }^{28}$ Filmetrics. Thin Film Measurement Technology Guide. 2012.

${ }^{29}$ Operations Manual for the Filmetrics F20 Thin-Film Analyzer. Filmetrics, Inc. 2007.

${ }^{30}$ J. Tauc (Ed.), Amorphous and Liquid Semiconductors, Plenum Press, New York, 1974, p. 211.

${ }^{31}$ Kumar, Vipin, Sachin Kr Sharma, T. P. Sharma, and V. Sing. "Band Gap Determination in Thick Films from Reflectance Measurements." Optical Materials 12.1 (1999): 115-19. Print. 32 Tauc, J. "Optical Properties." Amorphous and Liquid Semiconductors. London: Plenum, 1974. 159. Print.

${ }^{33}$ Gittleman, J., E. Sichel, and Y. Arie. "Composite Semiconductors: Selective Absorbers of Solar Energy." Solar Energy Materials 1.1-2 (1979): 93-104. Print.

${ }^{34}$ Srikant, V., and D. R. Clarke. "On the Optical Band Gap of Zinc Oxide." Journal of Applied Physics 83.10 (1998): 5447. Print.

${ }^{35}$ Tüzemen, Ebru Şenadım, Sıtkı Eker, Hamide Kavak, and Ramazan Esen. "Dependence of Film Thickness on the Structural and Optical Properties of ZnO Thin Films." Applied Surface Science 255.12 (2009): 6195-200. Print. 
${ }^{36}$ Schuetze, Andrew P., Wayne Lewis, Chris Brown, and Wilhelmus J. Geerts. "A Laboratory on the Four-point Probe Technique." American Journal of Physics 72.2 (2004): 149-53. American Journal of Physics. American Association of Physics Teachers. Web. 5 Apr. 2012. <http://ajp.aapt.org/resource/1/ajpias/v72/i2/p149_s1>.

37 "Kelvin (4-wire) Resistance Measurement." All About Circuits. Web. 05 Apr. 2012. <http://www.allaboutcircuits.com/vol_1/chpt_8/9.html>.

38 "AFM and Combined Optical Techniques." Asylum Research. Web. 06 Apr. 2012. <http://www.asylumresearch.com/Applications/CombinedAFMOptical/CombinedAFMOptical.shtm $1>$.

${ }^{39}$ Gannepalli, A., D. G. Yablon, A. H. Tsou, and R. Proksch. "Mapping Nanoscale Elasticity and Dissipation Using Dual Frequency Contact Resonance AFM." Nanotechnology: 1-14. Print. ${ }^{40}$ Buffered Oxide Etch; MSDS No. B5636 [Online]; Avantor Performance Materials, Inc.:Center Valley, PA, August 26, 2011, http://www.avantormaterials.com/documents/MSDS/usa/English/B5636 msds us cov Default.p df (Accessed April 17, 2012).

41 "Photolithography Resist Processes and Capabilities." Cornell NanoScale Science and Technology Facility. Web. 18 Apr. 2012.

$<$ http://www.cnf.cornell.edu/cnf_process_photo_resists.html>.

42 "Lithography." MEMS \& Nanotechnology Exchange. Web. 29 Apr. 2012. <https://www.memsexchange.org/MEMS/processes/lithography.html>.

${ }^{43}$ Duan, Xuefeng. "Microfabrication Using Bulk Wet Etching with TMAH." Thesis. McGill University, 2007. Web.

${ }^{44}$ O'Shea, S. J., R. M. Atta, and M. E. Welland. "Characterization of Tips for Conducting Atomic Force Microscopy." Review of Scientific Instruments 66.3 (1995): 2508-512. IEEE Explore Digital Library. American Institute of Physics. Web. 10 Jan. 2012.

<http://ieeexplore.ieee.org/xpl/freeabs_all.jsp?arnumber=4993002\&abstractAccess=no\&userType $=$ inst $>$. 
${ }^{45}$ Do, Dal-Hyun. Investigation of Ferroelectricity and Piezoelectricity in Ferroelectric Thin Film Capacitors Using Synchotron X-Ray Microdiffraction. Diss. University of Wisconsin-Madison, 2006. Print.

${ }^{46}$ Ko, Sang. "Micromachined Piezoelectric Membrane Acoustic Device." Sensors and Actuators A: Physical 103.1-2 (2003): 130-34. Print.

${ }^{47}$ Hsu, Tai-Ran. "Engineering Mechanics for Microsystems Design." MEMS and Microsystems: Design, Manufacture, and Nanoscale Engineering. 2nd ed. Hoboken, NJ: John Wiley, 2008. 109-81. Print.

${ }^{48}$ Alvarez, Brian Joel., and Richard N. Savage. Design, Fabrication, and Characterization of a Thin-film Nickel-titanium Shape Memory Alloy Diaphragm for Use in Micro-electro-mechanical Systems a Thesis. Thesis. California Polytechnic State University, 2011. DigitalCommons@ @al Poly. Web.

${ }^{49}$ Chen, J. "Effect of Sputtering Oxygen Partial Pressures on Structure and Physical Properties of High Resistivity ZnO Films." Applied Surface Science 223.4 (2004): 318-29. Print.

50 Schuler, Leo P., Nagarajan Valanoor, Paul Miller, lan Guy, Roger J. Reeves, and Maan M. Alkaisi. "The Effect of Substrate Material and Postannealing on the Photoluminescence and Piezo Properties of DC-Sputtered ZnO." Journal of Electronic Materials 36.4 (2007): 507-18. Print.

${ }^{51}$ SP4 Ordering Information. Gilroy, CA: Signatone, 2011. PDF.

${ }^{52}$ A.T. Santhanam, P. Tierney, and J.L. Hunt, Cemented Carbides, Properties and Selection: Nonferrous Alloys and Special-Purpose Materials, Vol 2, ASM Handbook, ASM International, 1990, p 950-977.

${ }^{53}$ Han, Cheol-Hyun, and Eun Sok Kim. "Parylene-Diaphragm Piezoelectric Acoustic Transducers." (2000): 148-52. Print.

54 Sernelius, B. E., Z.-C. Jin, I. Hamberg, and C. G. Granqvist. "Band-gap Tailoring of ZnO by Means of Heavy Al Doping." Physical Review B 37.17 (1988): 10244-0248. Print. 


\section{Appendices}

\section{Appendix A: XRD Data}

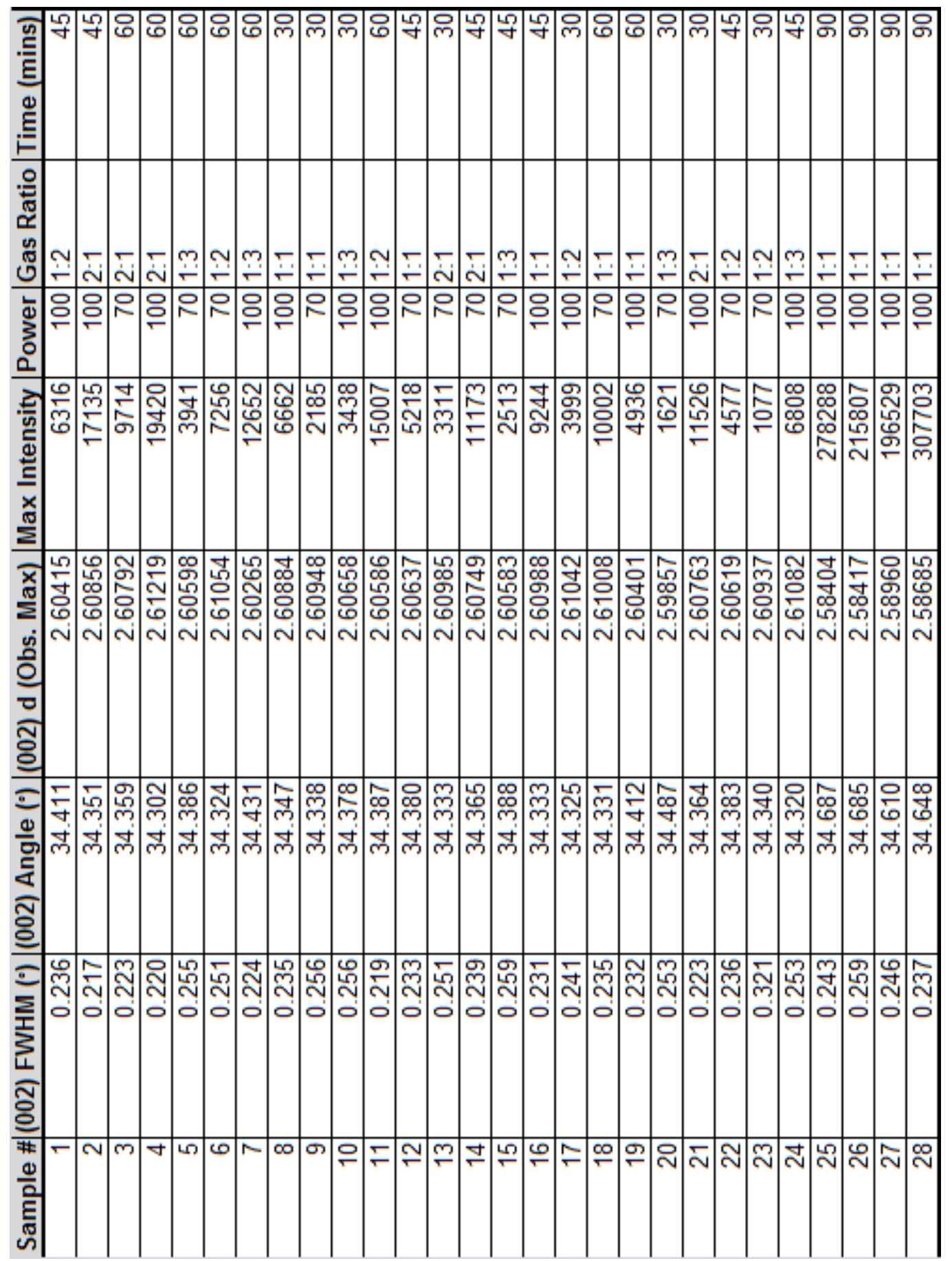




\section{Appendix B: ZnO Thickness Data}

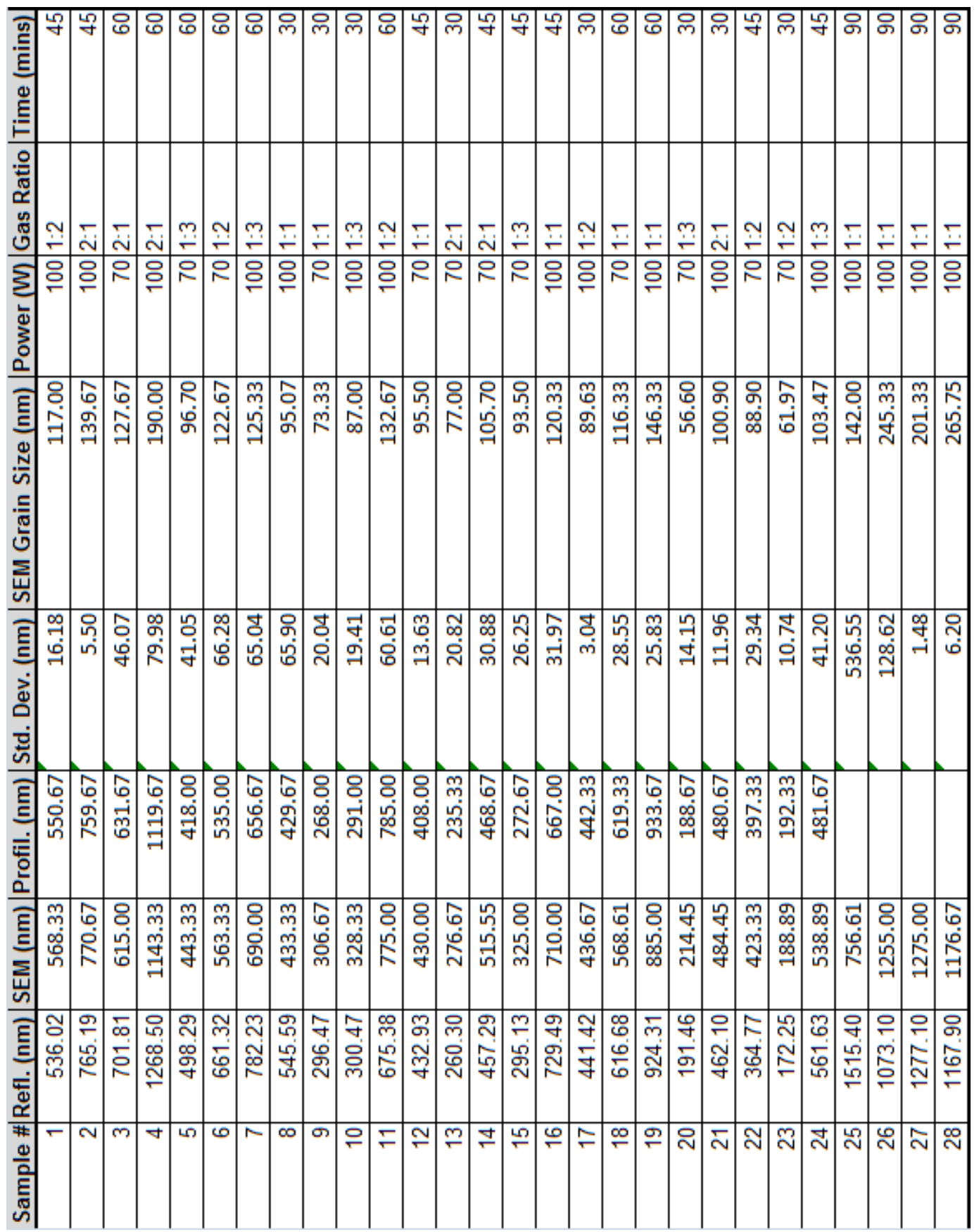




\section{Appendix C: $d_{33}$ Data}

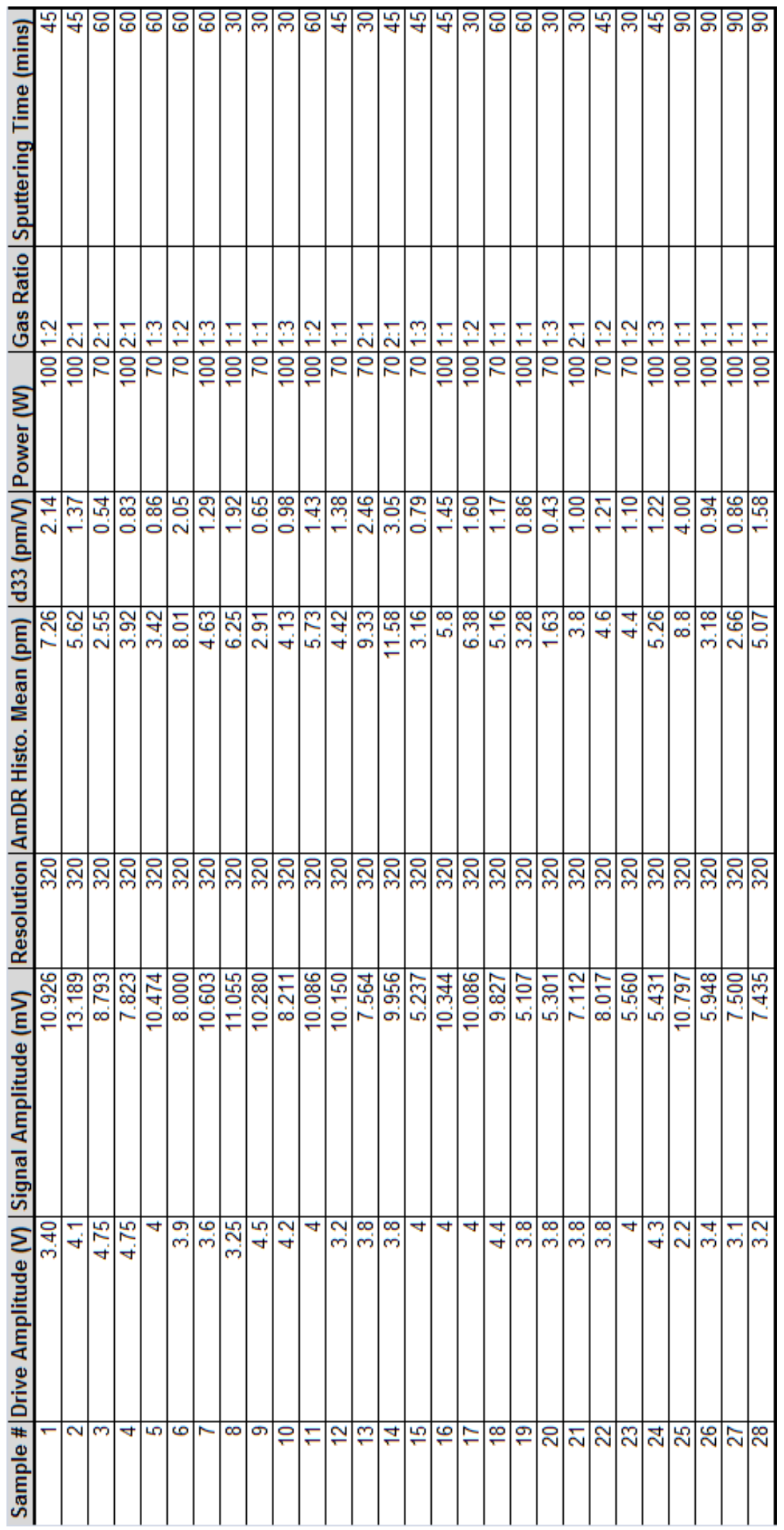




\section{Appendix D: Band Gap Data}

\begin{tabular}{|l|l|l|l|l|} 
Sample \# & Band Gap (eV) & Power (W) & Gas Ratio & Sputtering Time (mins) \\
\hline
\end{tabular}

\begin{tabular}{|c|c|c|c|c|}
\hline 1 & 2.80 & 100 & 1:2 & 45 \\
\hline 2 & 2.90 & 100 & 2:1 & 45 \\
\hline 3 & 2.85 & 70 & 2:1 & 60 \\
\hline 4 & 2.93 & 100 & 2:1 & 60 \\
\hline 5 & 2.75 & 70 & 1:3 & 60 \\
\hline 6 & 2.70 & 70 & 1:2 & 60 \\
\hline 7 & 2.60 & 100 & $1: 3$ & 60 \\
\hline 8 & 2.77 & 100 & 1:1 & 30 \\
\hline 9 & 2.80 & 70 & 1:1 & 30 \\
\hline 10 & 2.79 & 100 & 1:3 & 30 \\
\hline 11 & 2.85 & 100 & 1:2 & 60 \\
\hline 12 & 2.70 & 70 & 1:1 & 45 \\
\hline 13 & 2.62 & 70 & 2:1 & 30 \\
\hline 14 & 2.91 & 70 & 2:1 & 45 \\
\hline 15 & 2.75 & 70 & $1: 3$ & 45 \\
\hline 16 & 2.86 & 100 & 1:1 & 45 \\
\hline 17 & 2.92 & 100 & 1:2 & 30 \\
\hline 18 & 2.79 & 70 & 1:1 & 60 \\
\hline 19 & 2.68 & 100 & 1:1 & 60 \\
\hline 20 & 2.71 & 70 & $1: 3$ & 30 \\
\hline 21 & 2.80 & 100 & 2:1 & 30 \\
\hline 22 & 2.78 & 70 & $1: 2$ & 45 \\
\hline 23 & 2.75 & 70 & $1: 2$ & 30 \\
\hline 24 & 2.78 & 100 & 1:3 & 45 \\
\hline 25 & 2.90 & 100 & $1: 1$ & 90 \\
\hline 26 & 2.97 & 100 & $1: 1$ & 90 \\
\hline 27 & 3.15 & 100 & 1:1 & 90 \\
\hline 28 & 2.90 & 100 & 1:1 & 90 \\
\hline
\end{tabular}




\section{Appendix E: Resistivity Data, Annealed Samples}

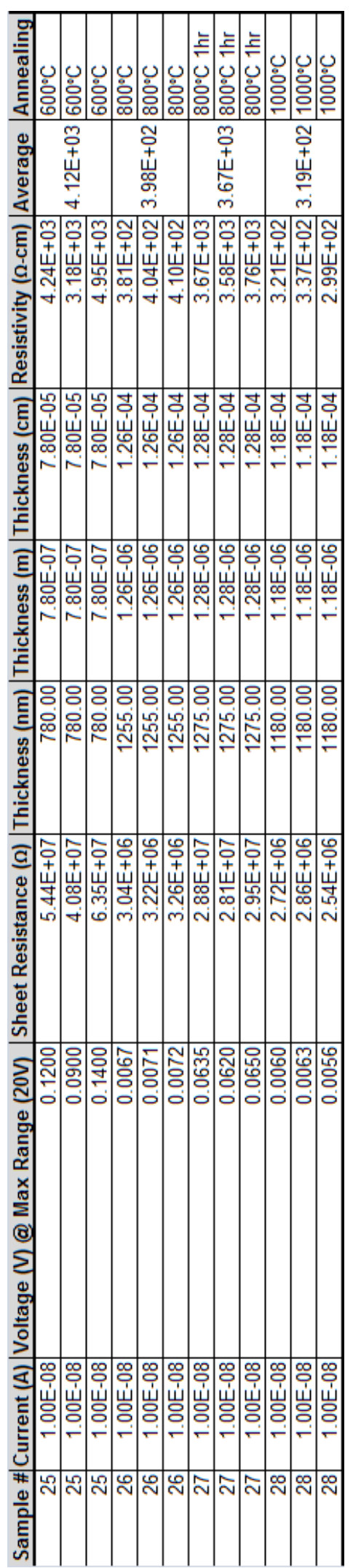

